\title{
Highly Enantioselective Transfer Hydrogenation of
}

\section{Polar Double Bonds by Macrocyclic Iron(II) / $(\mathrm{NH})_{2} \mathrm{P}_{2}$}

\section{Catalysts}

\author{
Raphael Bigler and Antonio Mezzetti* \\ Department of Chemistry and Applied Biosciences, ETH Zürich, CH-8093 Zürich, Switzerland
}

\section{Supplementary Material}

\section{Table of Contents}

General

S2

Synthesis of Dialdehyde $\left(S_{\mathrm{P}}, S_{\mathrm{P}}\right)-\mathbf{5}$

S4

Synthesis of Macrocycles and Iron(II) Complexes

Asymmetric Transfer Hydrogenation of Ketones and Imines 9

S48

References

S77 
General. Reactions with air- or moisture-sensitive materials were carried out under an argon atmosphere using Schlenk techniques or in a glove box under argon. All solvents were distilled from an appropriate drying agent under argon prior to use and used within one day (diethyl ether and THF from Na/benzophenone; hexane from $\mathrm{Na}$ /benzophenone/tetraglyme; EtOH from Na/ethyl phthalate; $\mathrm{CH}_{2} \mathrm{Cl}_{2}, \mathrm{CD}_{2} \mathrm{Cl}_{2}, \mathrm{MeCN}, \mathrm{MeOH}$ and 2-propanol from $\left.\mathrm{CaH}_{2}\right) \cdot{ }^{1} \mathrm{H},{ }^{13} \mathrm{C}$, and ${ }^{31} \mathrm{P} \mathrm{NMR}$ spectra were recorded on the following instruments: Bruker Avance DPX 300 $\left({ }^{1} \mathrm{H}, 300.1 ;{ }^{13} \mathrm{C}\left\{{ }^{1} \mathrm{H}\right\}, 75.5 ;{ }^{31} \mathrm{P}\left\{{ }^{1} \mathrm{H}\right\}, 121.5\right)$ and $500\left({ }^{1} \mathrm{H}, 500.2 ;{ }^{13} \mathrm{C}\left\{{ }^{1} \mathrm{H}\right\}, 125.8 ;{ }^{31} \mathrm{P}\left\{{ }^{1} \mathrm{H}\right\}, 202.5\right) .{ }^{1} \mathrm{H}$ and ${ }^{13} \mathrm{C}$ positive chemical shifts in ppm are downfield from tetramethylsilane. ${ }^{31} \mathrm{P}\left\{{ }^{1} \mathrm{H}\right\}$ NMR spectra are referenced to external $85 \% \mathrm{H}_{3} \mathrm{PO}_{4}$. For complexes, ${ }^{13} \mathrm{C}\left\{{ }^{31} \mathrm{P},{ }^{1} \mathrm{H}\right\}$ spectra were measured with ${ }^{31} \mathrm{P}$ (and ${ }^{1} \mathrm{H}$ ) decoupling to improve the $\mathrm{S} / \mathrm{N}$ ratio. Mass spectra were measured by the MS service of the Laboratory of Organic Chemistry (ETH Zürich). Elemental analyses were carried out by the Laboratory of Microelemental Analysis (ETH Zürich). Ketones 10a-g and 10i were distilled from $\mathrm{CaH}_{2}$ under reduced pressure prior to use. Ketone 10h was crystallized from hexane prior to use. Phosphinic amide $\mathbf{1 0 j}$ was crystallized from $\mathrm{CH}_{2} \mathrm{Cl}_{2} /$ hexane prior to use.

Borane dimethyl sulfide complex (94\%), (-)-menthol (99\%), tert-butyllithium (1.9 M in pentane), and titanium(IV) iso-propoxide were obtained from ABCR. 2-Bromobenzaldehyde was obtained from Apollo Scientific. Potassium hydroxide was obtained from Fisher. 1,8-Diazabicyclo[5.4.0]undec-7-ene was obtained from Fluorochem. $n$-Butyllithium (1.6 $\mathrm{M}$ in hexanes), diisopropylamine ( $\geq 99.5 \%)$, ethylene glycol ( $\geq 99 \%)$, formaldehyde ( $37 \%$ in water), iodomethane (99\%), iron(II) tetrafluoroborate hexahydrate (97\%), lithium aluminum hydride powder (95\%), poly(methylhydrosiloxane) (average $\left.\mathrm{M}_{\mathrm{n}}=1700-3200\right)$, pyridine (99.5\%), sodium borohydride ( $\geq 99.5 \%)$, sodium tert-butoxide $(97 \%)$, and $p$-toluenesulfonic acid monohydrate ( $\geq 98.5 \%)$ were obtained from Sigma-Aldrich. Dichlorophenylphosphine (>98\%), and (1S,2S)-(+)- 
1,2-cyclohexanediamine (>98\%) was obtained from TCI. Copper(II) chloride (97\%) was obtained from Sigma-Aldrich and dried at $100{ }^{\circ} \mathrm{C}$ under high vacuum for $12 \mathrm{~h}$ prior to use.

Thin layer chromatography was performed on Merck silica gel 60 F254 glass plates and visualized with UV fluorescence at $254 \mathrm{~nm}$ or stained in potassium permanganate solutions. Column chromatographic purifications were performed as flash column chromatography with $0.3-0.8$ bar overpressure using Silicycle silica gel (SiliaFlash ${ }^{\circledR}$ P60, $230-400$ mesh) as stationary phase. 


\section{Synthesis of Dialdehyde $\left(S_{\mathrm{P}}, S_{\mathrm{P}}\right)-5$}

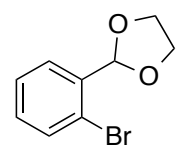

Synthesis of 2-(2-Bromophenyl)-1,3-dioxolane. The title compound was prepared following a literature procedure. ${ }^{\mathrm{S} 1}$ A $1000 \mathrm{~mL}$ Dean-Stark apparatus was charged with 2-bromobenzaldehyde (100.0 g, $540 \mathrm{mmol})$, ethylene glycol (37.7 mL, $676 \mathrm{mmol}, 1.25$ equiv), p-toluenesulfonic acid monohydrate $(2.06 \mathrm{~g}, 10.8 \mathrm{mmol}, 2 \mathrm{~mol} \%)$, and toluene $(540 \mathrm{~mL})$. After heating to reflux with continuous removal of water for $18 \mathrm{~h}$ and then cooling to room temperature, the solution was washed with $10 \%$ aqueous $\mathrm{NaOH}$ solution $(400 \mathrm{~mL}$ ) and saturated aqueous $\mathrm{NaCl}$ solution $(400 \mathrm{~mL})$, dried over $\mathrm{MgSO}_{4}$, filtered, and the solvent was removed at the rotary evaporator $\left(20 \mathrm{mbar}, 60^{\circ} \mathrm{C}\right)$. Purification by distillation under reduced pressure $\left(10^{-1} \mathrm{mbar}, 105^{\circ} \mathrm{C}\right)$ afforded the product as a clear liquid. Yield: $121.2 \mathrm{~g}(98 \%) .{ }^{1} \mathbf{H}$ NMR $\left(300 \mathrm{MHz}, \mathrm{CDCl}_{3}\right): \delta 7.61$ $\left(d d,{ }^{3} J_{\mathrm{H}, \mathrm{H}^{\prime}}=7.7 \mathrm{~Hz},{ }^{4} J_{\mathrm{H}, \mathrm{H}^{\prime}}=1.8 \mathrm{~Hz}, 1 \mathrm{H}, \operatorname{Ar}-H\right), 7.57\left(d d,{ }^{3} J_{\mathrm{H}, \mathrm{H}^{\prime}}=7.9 \mathrm{~Hz},{ }^{4} J_{\mathrm{H}, \mathrm{H}^{\prime}}=1.3 \mathrm{~Hz}, 1 \mathrm{H}\right.$, $\operatorname{Ar}-H), 7.34\left(d d d,{ }^{3} J_{\mathrm{H}, \mathrm{H}^{\prime}}=7.7,7.3 \mathrm{~Hz},{ }^{4} J_{\mathrm{H}, \mathrm{H}^{\prime}}=1.3 \mathrm{~Hz}, 1 \mathrm{H}, \operatorname{Ar}-H\right), 7.22\left(d d d,{ }^{3} J_{\mathrm{H}, \mathrm{H}^{\prime}}=7.9,7.3 \mathrm{~Hz}\right.$, $\left.{ }^{4} J_{\mathrm{H}, \mathrm{H}^{\prime}}=1.8 \mathrm{~Hz}, 1 \mathrm{H}, \mathrm{Ar}-H\right), 6.11\left(s, 1 \mathrm{H}, \mathrm{CH}\left(\mathrm{OCH}_{2}\right)_{2}\right), 4.20-4.11(m, 2 \mathrm{H}, \mathrm{OC} H \mathrm{H}), 4.11-4.02(m$, 2H, OCHH). ${ }^{13} \mathbf{C}\left\{{ }^{1} \mathbf{H}\right\}$ NMR (75 MHz, $\mathrm{CDCl}_{3}$ ): $\delta 136.7$ (arom.), 133.1 (arom.), 130.7 (arom.), 127.9 (arom.), 127.5 (arom.), 123.0 (arom.), $102.7\left(\mathrm{CH}\left(\mathrm{OCH}_{2}\right)_{2}\right), 65.6\left(\mathrm{OCH}_{2}\right)$. IR (liquid film, $\left.\mathrm{cm}^{-1}\right)$ : 3067 (C-H), 2954 (C-H), 2885 (C-H), 1592, 1571, 1470, 1443, 1385, 1270, 1210, 1160, 1124, 1082, 1041, 1020. HRMS (EI): Calcd. for $\mathrm{C}_{9} \mathrm{H}_{8} \mathrm{BrO}_{2} \mathrm{~m} / \mathrm{z}=226.9703$, found $\mathrm{m} / \mathrm{z}=226.9704$ $[\mathrm{M}-\mathrm{H}]^{+}$. EA: Calcd. for $\mathrm{C}_{9} \mathrm{H}_{9} \mathrm{BrO}_{2}: \mathrm{C}, 47.19 ; \mathrm{H}, 3.96$; found: C, 47.13; H, 4.14. 
Figure S1: ${ }^{1} \mathrm{H}$ NMR spectrum of 2-(2-bromophenyl)-1,3-dioxolane (300 $\left.\mathrm{MHz}, \mathrm{CDCl}_{3}\right)$.

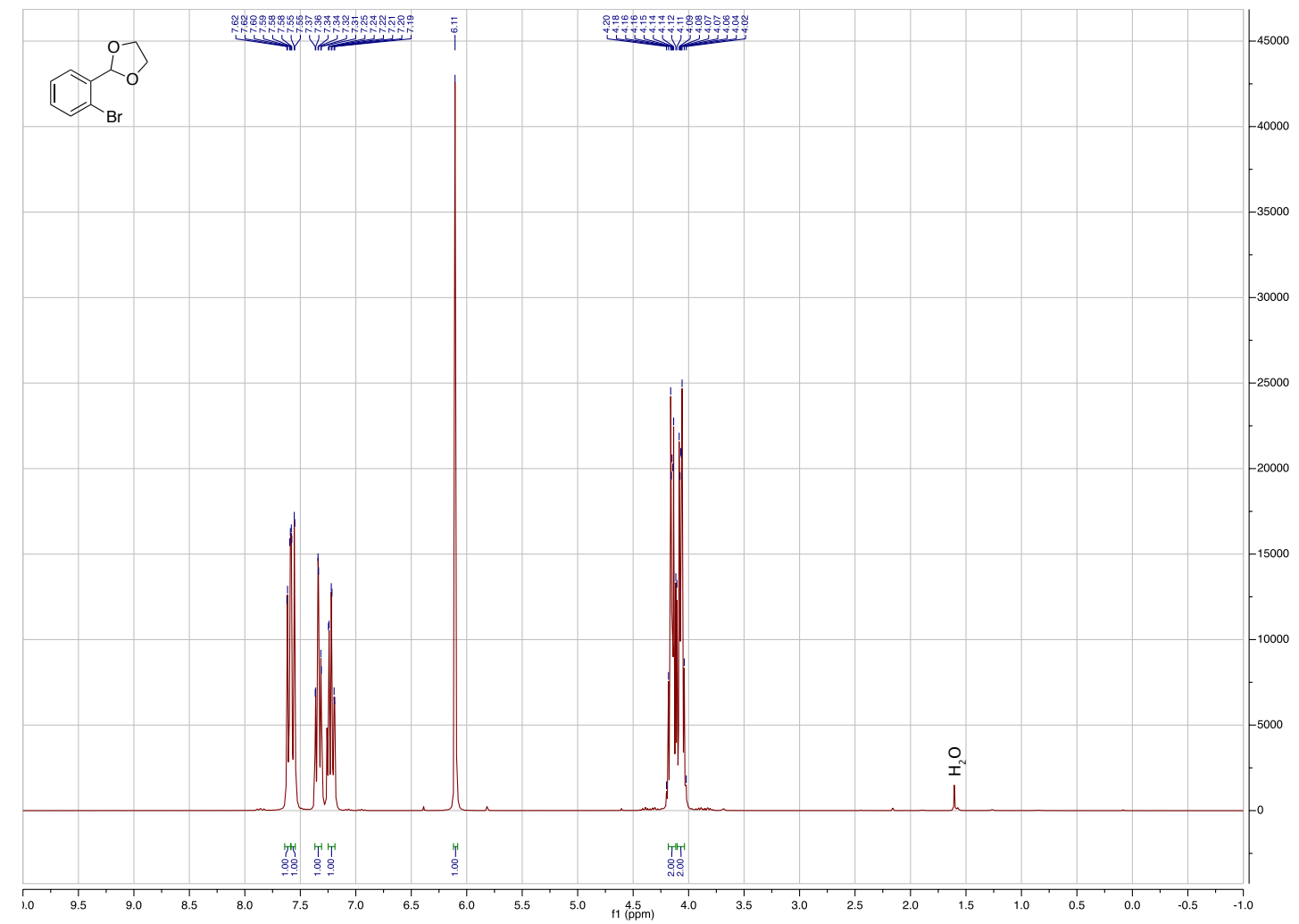

Figure S2: ${ }^{13} \mathrm{C}\left\{{ }^{1} \mathrm{H}\right\}$ NMR spectrum of 2-(2-bromophenyl)-1,3-dioxolane (75 $\mathrm{MHz}, \mathrm{CDCl}_{3}$ ).

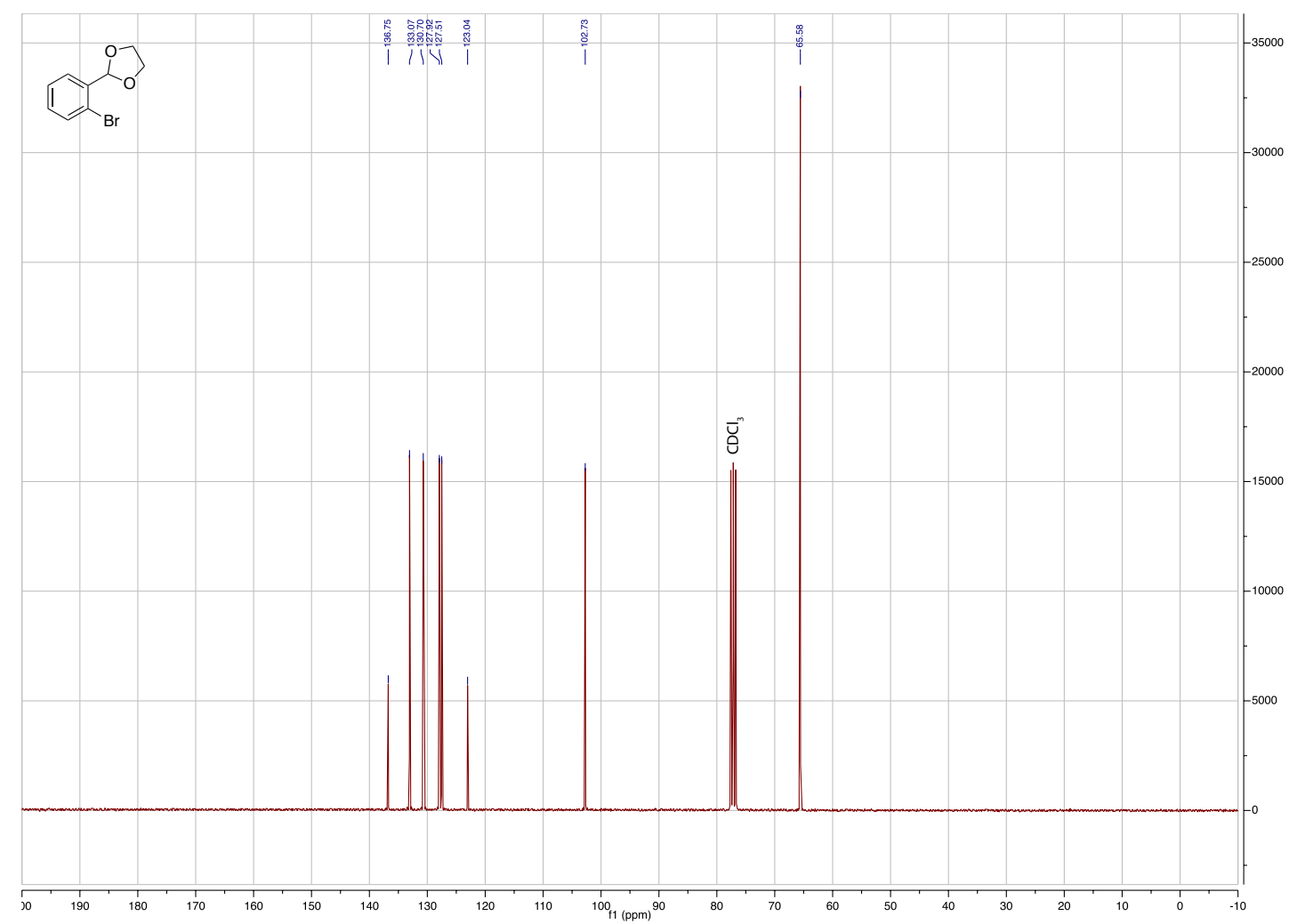




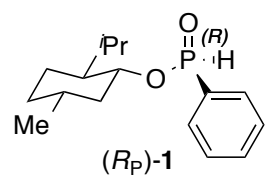

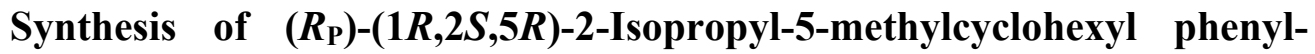

phosphinate, $\left(\boldsymbol{R}_{\mathrm{P}}\right)-\mathbf{1}$. The title compound was prepared following a literature procedure. ${ }^{\mathrm{S} 2}$ In a flame-dried $1000 \mathrm{~mL}$ two-neck round-bottom flask, (-)-menthol (100.0 g, $640 \mathrm{mmol})$ and pyridine (51.8 mL, $640 \mathrm{mmol}, 1.0$ equiv) were dissolved in diethyl ether $(400 \mathrm{~mL})$. In a separate $2000 \mathrm{~mL}$ two-neck round-bottom flask, dichloro(phenyl)phosphine $(86.8 \mathrm{~mL}, 640 \mathrm{mmol}, 1.0$ equiv) was dissolved in diethyl ether $(400 \mathrm{~mL})$ and cooled to $0{ }^{\circ} \mathrm{C}$. The first solution was then added dropwise over $1.5 \mathrm{~h}$ using a cannula and warmed to room temperature overnight. The resulting suspension was filtered with diethyl ether $(500 \mathrm{~mL})$ into a $2000 \mathrm{~mL}$ round-bottom flask to remove pyridine hydrochloride, cooled to $-78{ }^{\circ} \mathrm{C}$, and water $(500 \mathrm{~mL})$ was added dropwise over $4 \mathrm{~h}$ (Note: The hydrolysis of the intermediate chlorophosphine is conducted in air). After warming to room temperature, the organic phase was removed at the rotary evaporator $\left(600 \mathrm{mbar}, 40{ }^{\circ} \mathrm{C}\right)$. Hexane $(1000 \mathrm{~mL})$ was added, and the aqueous phase was separated. The organic phase was washed twice with saturated aqueous $\mathrm{Na}_{2} \mathrm{CO}_{3}$ solution $(2 \times 500 \mathrm{~mL})$, dried over $\mathrm{MgSO}_{4}$, filtered, and the solvent was removed at the rotary evaporator $\left(20\right.$ mbar, $\left.40{ }^{\circ} \mathrm{C}\right)$. The crude product $\left({ }^{31} \mathrm{P}\left\{{ }^{1} \mathrm{H}\right\}\right.$ NMR: $24.6(78 \%), 21.2(22 \%))$ was recrystallized four times from hexane $(4 \times 25 \mathrm{~mL})$ at $-23{ }^{\circ} \mathrm{C}$ to give the product as a clear solid. A single diastereoisomer (d.r. $=570: 1)$ was obtained as judged by the ${ }^{31} \mathrm{P}\left\{{ }^{1} \mathrm{H}\right\}$ NMR spectrum. Yield: $61.4 \mathrm{~g}(34 \%) .{ }^{1} \mathbf{H}$ NMR $\left(300 \mathrm{MHz}, \mathrm{CDCl}_{3}\right): \delta 7.80-7.71(\mathrm{~m}, 2 \mathrm{H}$, $\operatorname{Ar}-H), 7.63\left(d,{ }^{1} J_{\mathrm{P}, \mathrm{H}}=552.8 \mathrm{~Hz}, 1 \mathrm{H}, \mathrm{P}-H\right), 7.60-7.53(m, 1 \mathrm{H}, \mathrm{Ar}-H), 7.52-7.44(m, 2 \mathrm{H}, \mathrm{Ar}-H)$, $4.33-4.19(m, 1 \mathrm{H}, \mathrm{CHO}), 2.25-2.10(m, 2 \mathrm{H}, \mathrm{CH}+\mathrm{CHH}), 1.73-1.60(m, 2 \mathrm{H}, \mathrm{CH}), 1.52-1.36$ $(m, 2 \mathrm{H}, \mathrm{CH}), 1.29-1.15(m, 1 \mathrm{H}, \mathrm{CHH}), 1.10-0.97(m, 2 \mathrm{H}, \mathrm{CHH}), 0.94\left(d,{ }^{3} J_{\mathrm{H}, \mathrm{H}}{ }^{\prime}=7.0 \mathrm{~Hz}, 3 \mathrm{H}\right.$, $\left.\mathrm{CH}_{3}\right), 0.87\left(d,{ }^{3} \mathrm{~J}_{\mathrm{H}, \mathrm{H}^{\prime}}=6.5 \mathrm{~Hz}, 3 \mathrm{H}, \mathrm{CH}\right), 0.84\left(d,{ }^{3} J_{\mathrm{H}, \mathrm{H}^{\prime}}=7.9 \mathrm{~Hz}, 3 \mathrm{H}, \mathrm{CH}_{3}\right) .{ }^{31} \mathbf{P}\left\{{ }^{1} \mathbf{H}\right\} \mathbf{N M R}$ $\left(122 \mathrm{MHz}, \mathrm{CDCl}_{3}\right): \delta 24.6(s) .{ }^{31} \mathbf{P}$ NMR $\left(122 \mathrm{MHz}, \mathrm{CDCl}_{3}\right): \delta 24.6\left(d t d,{ }^{1} J_{\mathrm{P}, \mathrm{H}}=552.8 \mathrm{~Hz},{ }^{3} J_{\mathrm{P}, \mathrm{H}}=\right.$ 
14.1, $10.4 \mathrm{~Hz}) .{ }^{13} \mathbf{C}\left\{{ }^{1} \mathbf{H}\right\}$ NMR $\left(75 \mathrm{MHz}, \mathrm{CDCl}_{3}\right): \delta 133.0\left(d,{ }^{4} J_{\mathrm{P}, \mathrm{C}}=3.0 \mathrm{~Hz}\right.$, arom. $), 131.1(d$, ${ }^{1} J_{\mathrm{P}, \mathrm{C}}=135.1 \mathrm{~Hz}$, arom. $), 130.7\left(d,{ }^{3} J_{\mathrm{P}, \mathrm{C}}=11.8 \mathrm{~Hz}\right.$, arom. $), 128.7\left(d,{ }^{2} J_{\mathrm{P}, \mathrm{C}}=14.0 \mathrm{~Hz}, \operatorname{arom}.\right), 79.0(d$, $\left.{ }^{2} J_{\mathrm{P}, \mathrm{C}}=7.2 \mathrm{~Hz}, \mathrm{CHO}\right), 48.8\left(d,{ }^{3} J_{\mathrm{P}, \mathrm{C}}=6.2 \mathrm{~Hz}, \mathrm{CH}\right), 43.6\left(d,{ }^{3} \mathrm{~J}_{\mathrm{P}, \mathrm{C}}=1.2 \mathrm{~Hz}, \mathrm{CH}_{2}\right), 34.0\left(\mathrm{CH}_{2}\right), 31.7$ $(\mathrm{CH}), 25.9(\mathrm{CH}), 23.0\left(\mathrm{CH}_{2}\right), 21.9\left(\mathrm{CH}_{3}\right), 21.1\left(\mathrm{CH}_{3}\right), 15.9\left(\mathrm{CH}_{3}\right)$. Melting Point: $31{ }^{\circ} \mathrm{C}$. IR (liquid film, cm ${ }^{-1}$ ): 3481 (P-H), 3058 (C-H), 2954 (C-H), 2924 (C-H), 2869 (C-H), 2337 (P=O), $1592,1456,1439,1388,1370,1348,1275,1230,1181,1126,1097,1059,1025$. HRMS (EI): Calcd. for $\mathrm{C}_{13} \mathrm{H}_{18} \mathrm{O}_{2} \mathrm{P} m / z=237.1039$, found $m / z=237.1038\left[\mathrm{M}-\mathrm{C}_{3} \mathrm{H}_{7}\right]^{+}$. EA: Calcd. for $\mathrm{C}_{16} \mathrm{H}_{25} \mathrm{O}_{2} \mathrm{P}: \mathrm{C}, 68.55 ; \mathrm{H}, 8.99$; found: $\mathrm{C}, 68.43 ; \mathrm{H}, 9.14$. Analytical data are in agreement with literature data. ${ }^{\mathrm{S} 2}$

Figure S3: ${ }^{1} \mathrm{H}$ NMR spectrum of $\left(R_{\mathrm{P}}\right)-\mathbf{1}\left(300 \mathrm{MHz}, \mathrm{CDCl}_{3}\right)$.

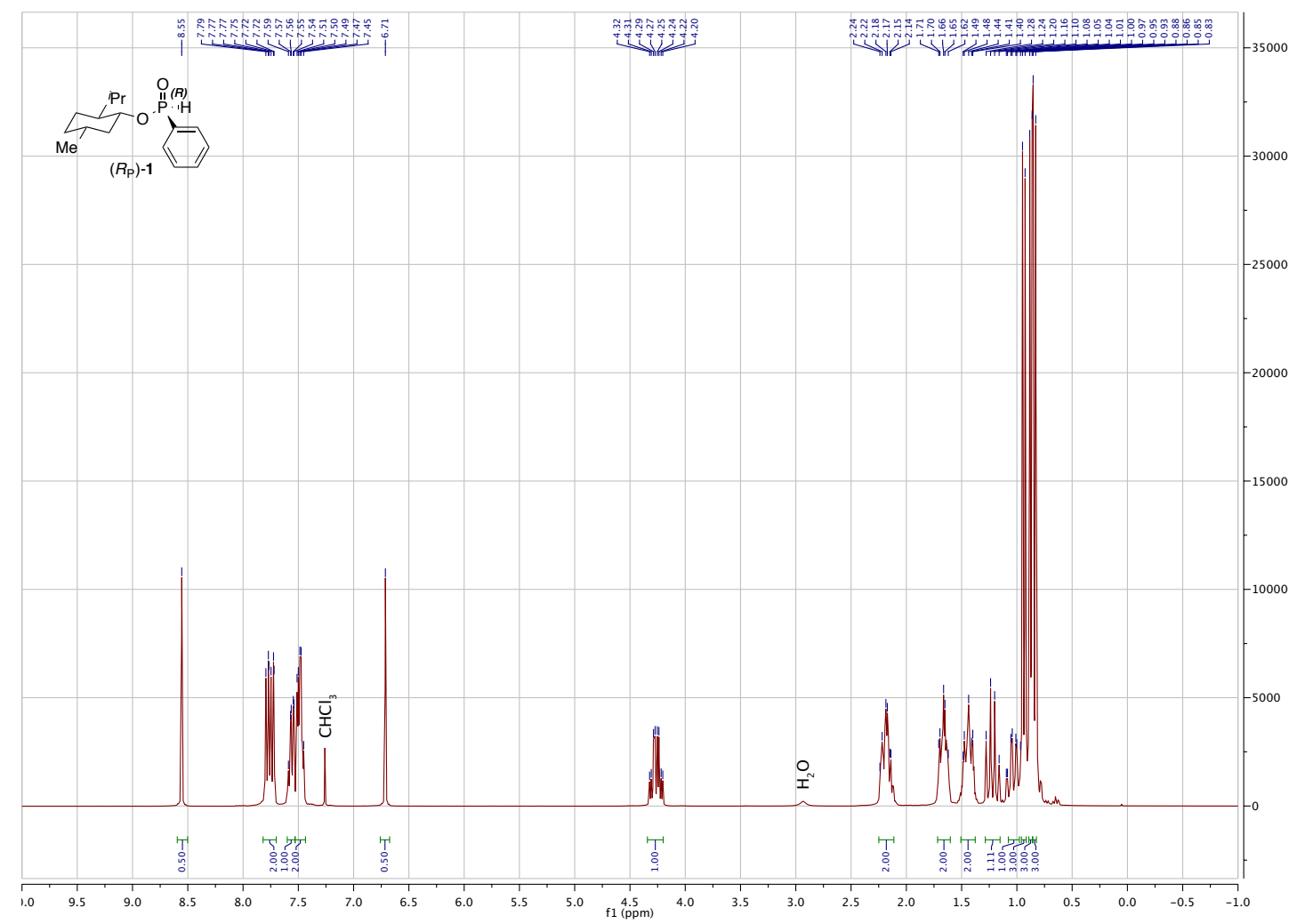


Figure S4: ${ }^{31} \mathrm{P}\left\{{ }^{1} \mathrm{H}\right\}$ NMR spectrum of $\left(R_{\mathrm{P}}\right)-\mathbf{1}\left(122 \mathrm{MHz}, \mathrm{CDCl}_{3}\right)$.

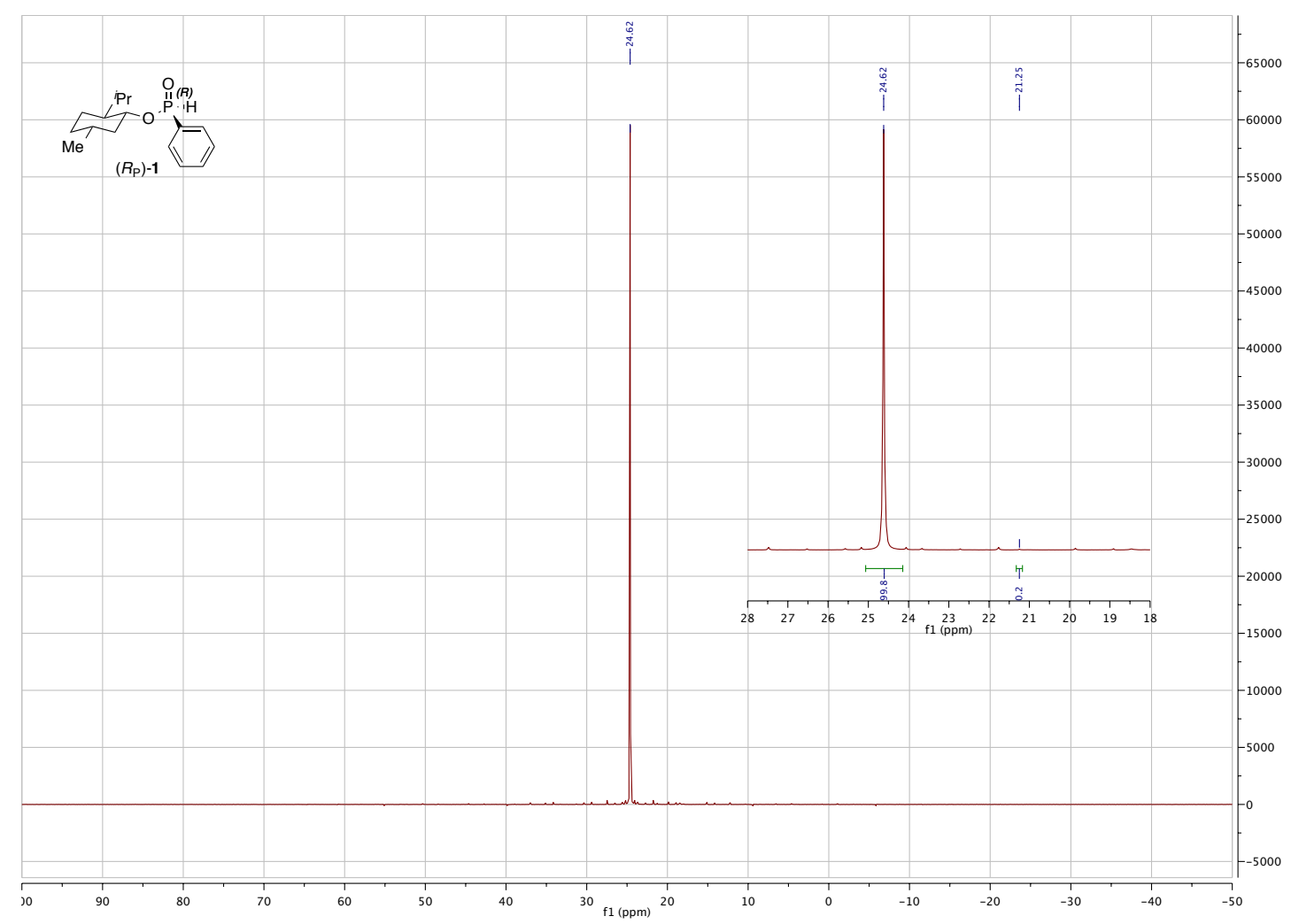

Figure S5: ${ }^{31} \mathrm{P}$ NMR spectrum of $\left(R_{\mathrm{P}}\right)-\mathbf{1}\left(122 \mathrm{MHz}, \mathrm{CDCl}_{3}\right)$.

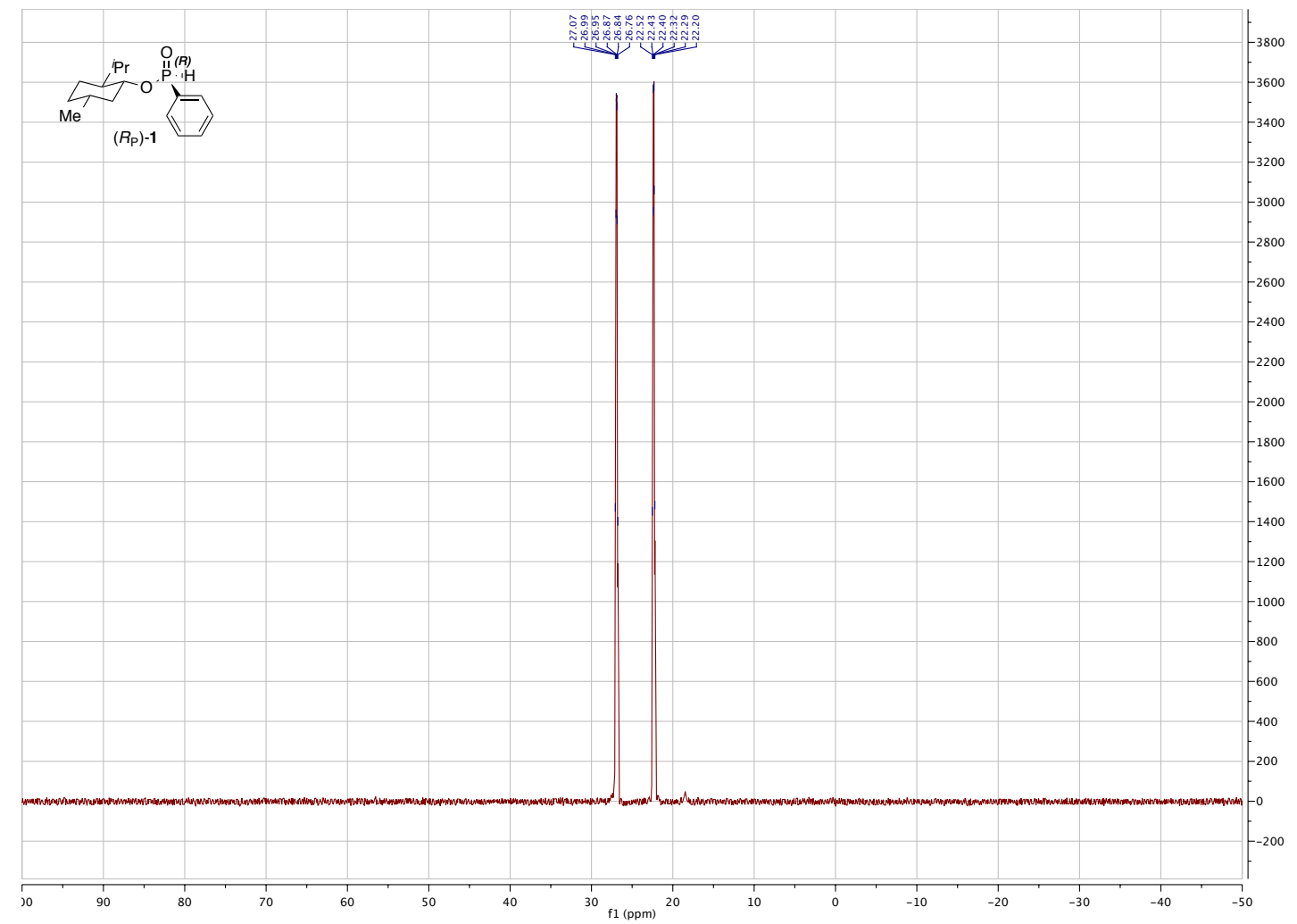


Figure S6: ${ }^{13} \mathrm{C}\left\{{ }^{1} \mathrm{H}\right\}$ NMR spectrum of $\left(R_{\mathrm{P}}\right)-\mathbf{1}\left(75 \mathrm{MHz}, \mathrm{CDCl}_{3}\right)$.
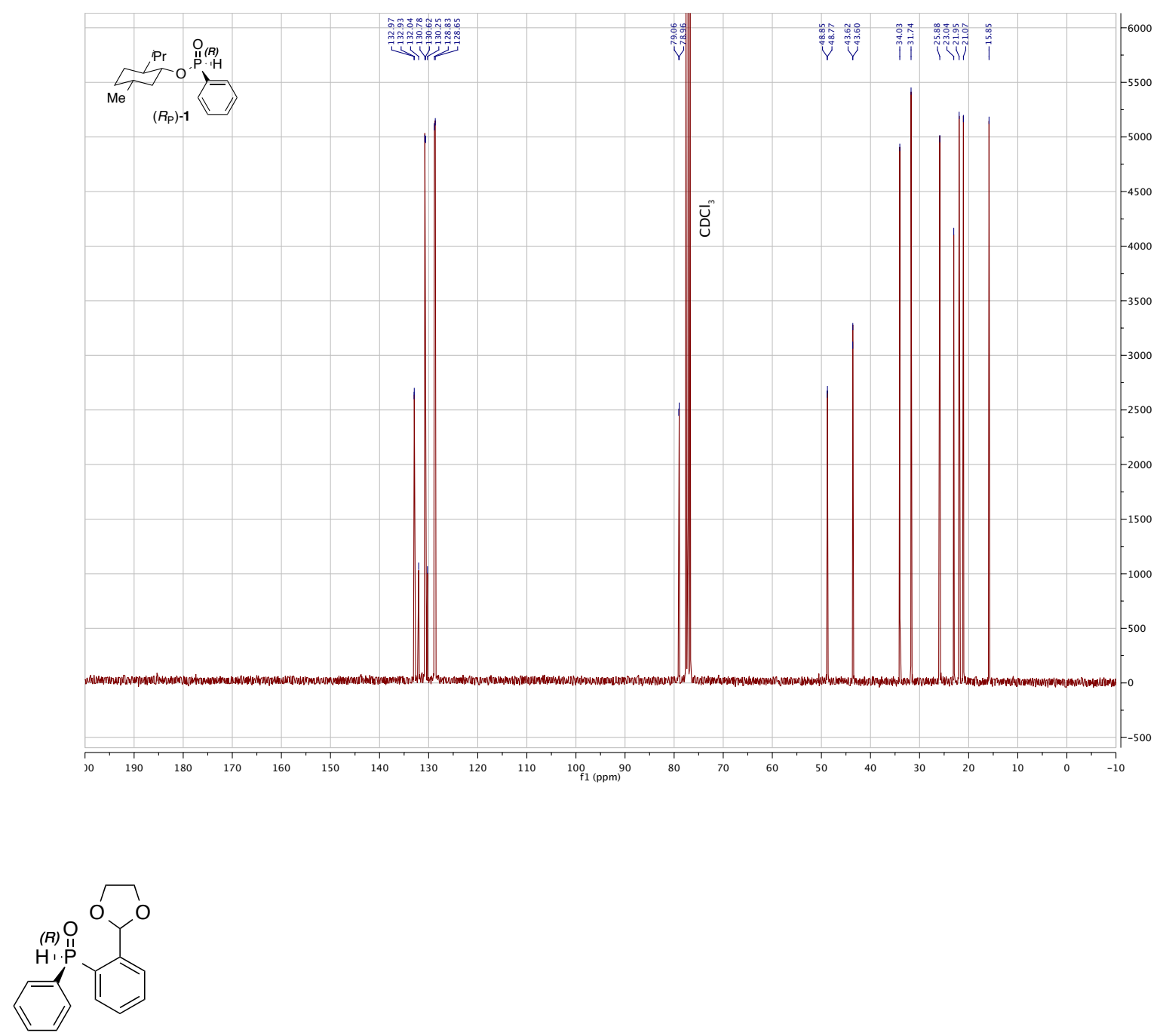

$\left(R_{\mathrm{P}}\right)-2$

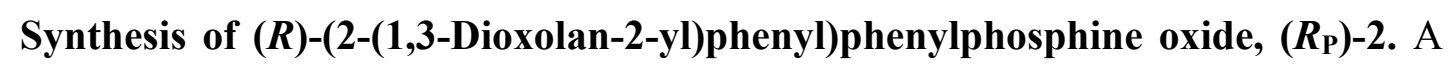
$2000 \mathrm{~mL}$ two-neck round-bottom flask was charged with 2-(2-bromophenyl)-1,3-dioxolane (28.4 mL, $190 \mathrm{mmol}, 2.1$ equiv) and diethyl ether $(570 \mathrm{~mL})$, and then cooled to $-78{ }^{\circ} \mathrm{C}$. After $10 \mathrm{~min}$, tert-butyllithium (200 mL, $1.9 \mathrm{M}$ in pentane, $380 \mathrm{mmol}$, 4.2 equiv) was added dropwise over $30 \mathrm{~min}$. After $10 \mathrm{~min}$, the solution was warmed to $0{ }^{\circ} \mathrm{C}$ for $1.5 \mathrm{~h}$ and then cooled to $-78^{\circ} \mathrm{C}$. In a $250 \mathrm{~mL}$ Schlenk flask, $\left(R_{\mathrm{P}}\right)-\mathbf{1}(25.4 \mathrm{~g}, 90 \mathrm{mmol})$ was dissolved in diethyl ether $(110 \mathrm{~mL})$ and added dropwise to the content of the first flask over 30 min using a cannula, and then the suspension was warmed to room temperature overnight. Saturated aqueous $\mathrm{NH}_{4} \mathrm{Cl}$ solution $(400 \mathrm{~mL})$ was added and, after $15 \mathrm{~min}$, the organic solvent was removed at the rotary evaporator 
$\left(600 \mathrm{mbar}, 40{ }^{\circ} \mathrm{C}\right)$. The biphasic solution was transferred with hexane $(400 \mathrm{~mL})$, water $(500 \mathrm{~mL})$, and saturated aqueous $\mathrm{NH}_{4} \mathrm{Cl}$ solution $(100 \mathrm{~mL})$ to a separatory funnel, and the organic phase was separated (Note: The product partially separated as a yellow oil, but was kept with the aqueous phase). The aqueous phase was washed four times with hexane $(4 \times 400 \mathrm{~mL})$ and extracted four times with chloroform $(4 \times 400 \mathrm{~mL})$. The combined chloroform phases were dried over $\mathrm{MgSO}_{4}$, filtered, and then the solvent was removed at the rotary evaporator $\left(20 \mathrm{mbar}, 40^{\circ} \mathrm{C}\right)$ to afford the product as a slightly yellowish oil, which was used without further purification (99\% pure by ${ }^{31} \mathrm{P}\left\{{ }^{1} \mathrm{H}\right\}$ NMR; Note: If impure product is obtained, it can be purified by flash column chromatography on silica gel $\left(\mathrm{CH}_{2} \mathrm{Cl}_{2}: \mathrm{MeOH}=100: 0\right.$ to $\left.\left.96: 4\right)\right)$. Yield: $22.1 \mathrm{~g}(89 \%, 99 \%$ ee $)$. ${ }^{1} \mathbf{H}$ NMR $\left(300 \mathrm{MHz}, \mathrm{CDCl}_{3}\right): \delta 8.34\left(d,{ }^{1} J_{\mathrm{P}, \mathrm{H}}=502.2 \mathrm{~Hz}, 1 \mathrm{H}, \mathrm{P}-H\right), 7.86\left(d d d,{ }^{3} J_{\mathrm{P}, \mathrm{H}}=15.2 \mathrm{~Hz}\right.$, $\left.{ }^{3} J_{\mathrm{H}, \mathrm{H}^{\prime}}=7.5 \mathrm{~Hz},{ }^{4} J_{\mathrm{H}, \mathrm{H}^{\prime}}=1.5 \mathrm{~Hz}, 1 \mathrm{H}, \mathrm{Ar}-H\right), 7.73-7.63(m, 3 \mathrm{H}, \mathrm{Ar}-H), 7.58\left(t t,{ }^{3} J_{\mathrm{H}, \mathrm{H}^{\prime}}=7.6 \mathrm{~Hz}\right.$, $\left.{ }^{4} J_{\mathrm{H}, \mathrm{H}^{\prime}}=1.6 \mathrm{~Hz}, 1 \mathrm{H}, \mathrm{Ar}-H\right), 7.55-7.42(m, 4 \mathrm{H}, \mathrm{Ar}-H), 6.05\left(s, 1 \mathrm{H}, \mathrm{CH}\left(\mathrm{OCH}_{2}\right)_{2}\right), 4.00-3.89(m$, 4H, $\left.\mathrm{CH}(\mathrm{OCHH})_{2}\right) \cdot{ }^{31} \mathbf{P}\left\{{ }^{1} \mathbf{H}\right\}$ NMR $\left(122 \mathrm{MHz}, \mathrm{CDCl}_{3}\right): \delta 19.0(s) .{ }^{31} \mathbf{P}$ NMR $\left(122 \mathrm{MHz}, \mathrm{CDCl}_{3}\right)$ : $\delta 19.0\left(d d t,{ }^{1} J_{\mathrm{P}, \mathrm{H}}=502.2 \mathrm{~Hz},{ }^{3} J_{\mathrm{P}, \mathrm{H}}=15.2,14.9 \mathrm{~Hz}\right) .{ }^{13} \mathbf{C}\left\{{ }^{1} \mathbf{H}\right\} \mathbf{N M R}\left(75 \mathrm{MHz}, \mathrm{CDCl}_{3}\right): \delta 140.8(d$, ${ }^{2} J_{\mathrm{P}, \mathrm{C}}=7.5 \mathrm{~Hz}$, arom. $), 132.9\left(d,{ }^{2} J_{\mathrm{P}, \mathrm{C}}=10.5 \mathrm{~Hz}\right.$, arom. $), 132.5\left(d,{ }^{4} J_{\mathrm{P}, \mathrm{C}}=2.7 \mathrm{~Hz}\right.$, arom. $), 132.4(d$, ${ }^{1} J_{\mathrm{P}, \mathrm{C}}=102.7 \mathrm{~Hz}$, arom. $), 132.2\left(d,{ }^{4} J_{\mathrm{P}, \mathrm{C}}=2.9 \mathrm{~Hz}\right.$, arom. $), 130.8\left(d,{ }^{2} J_{\mathrm{P}, \mathrm{C}}=11.4 \mathrm{~Hz}\right.$, arom. $), 129.9(d$, ${ }^{1} J_{\mathrm{P}, \mathrm{C}}=96.1 \mathrm{~Hz}$, arom. $), 129.3\left(d,{ }^{3} J_{\mathrm{P}, \mathrm{C}}=12.4 \mathrm{~Hz}\right.$, arom. $), 128.7\left(d,{ }^{3} J_{\mathrm{P}, \mathrm{C}}=12.9 \mathrm{~Hz}\right.$, arom. $), 127.3(d$, ${ }^{3} J_{\mathrm{P}, \mathrm{C}}=9.7 \mathrm{~Hz}$, arom. $), 101.6\left(d,{ }^{3} J_{\mathrm{P}, \mathrm{C}}=4.4 \mathrm{~Hz}, \mathrm{CH}\left(\mathrm{OCH}_{2}\right)_{2}\right), 65.1\left(\mathrm{CH}\left(\mathrm{OCH}_{2}\right)_{2}\right), 65.0$ $\left(\mathrm{CH}\left(\mathrm{OCH}_{2}\right)_{2}\right)$. IR (liquid film, cm $\left.{ }^{-1}\right): 3058(\mathrm{C}-\mathrm{H}), 2955(\mathrm{C}-\mathrm{H}), 2892(\mathrm{C}-\mathrm{H}), 2334(\mathrm{P}=\mathrm{O}), 1645$, 1591, 1573, 1483, 1474, 1438, 1398, 1347, 1311, 1293, 1177, 1134, 1113, 1096, 1065, 1038, 1023. HRMS (ESI): Calcd. for $\mathrm{C}_{15} \mathrm{H}_{16} \mathrm{O}_{3} \mathrm{P} m / z=275.0832$, found $\mathrm{m} / \mathrm{z}=275.0837[\mathrm{M}+\mathrm{H}]^{+}$. HPLC: Chiralpak IB-3 (hexane : 2-PrOH $=80: 20$, flow rate $1.0 \mathrm{~mL} / \mathrm{min}, \lambda=230 \mathrm{~nm}$ ), retention times $t_{\mathrm{R}}($ minor $)=13.5 \mathrm{~min}, t_{\mathrm{R}}($ major $)=19.1 \mathrm{~min} ; 99 \%$ ee. $[\boldsymbol{\alpha}]_{\mathbf{D}}{ }^{20}:+40.5\left(\mathrm{c}=1.0, \mathrm{CH}_{2} \mathrm{Cl}_{2}\right)$. 
Figure S7: ${ }^{1} \mathrm{H}$ NMR spectrum of $\left(R_{\mathrm{P}}\right)-2\left(300 \mathrm{MHz}, \mathrm{CDCl}_{3}\right)$.

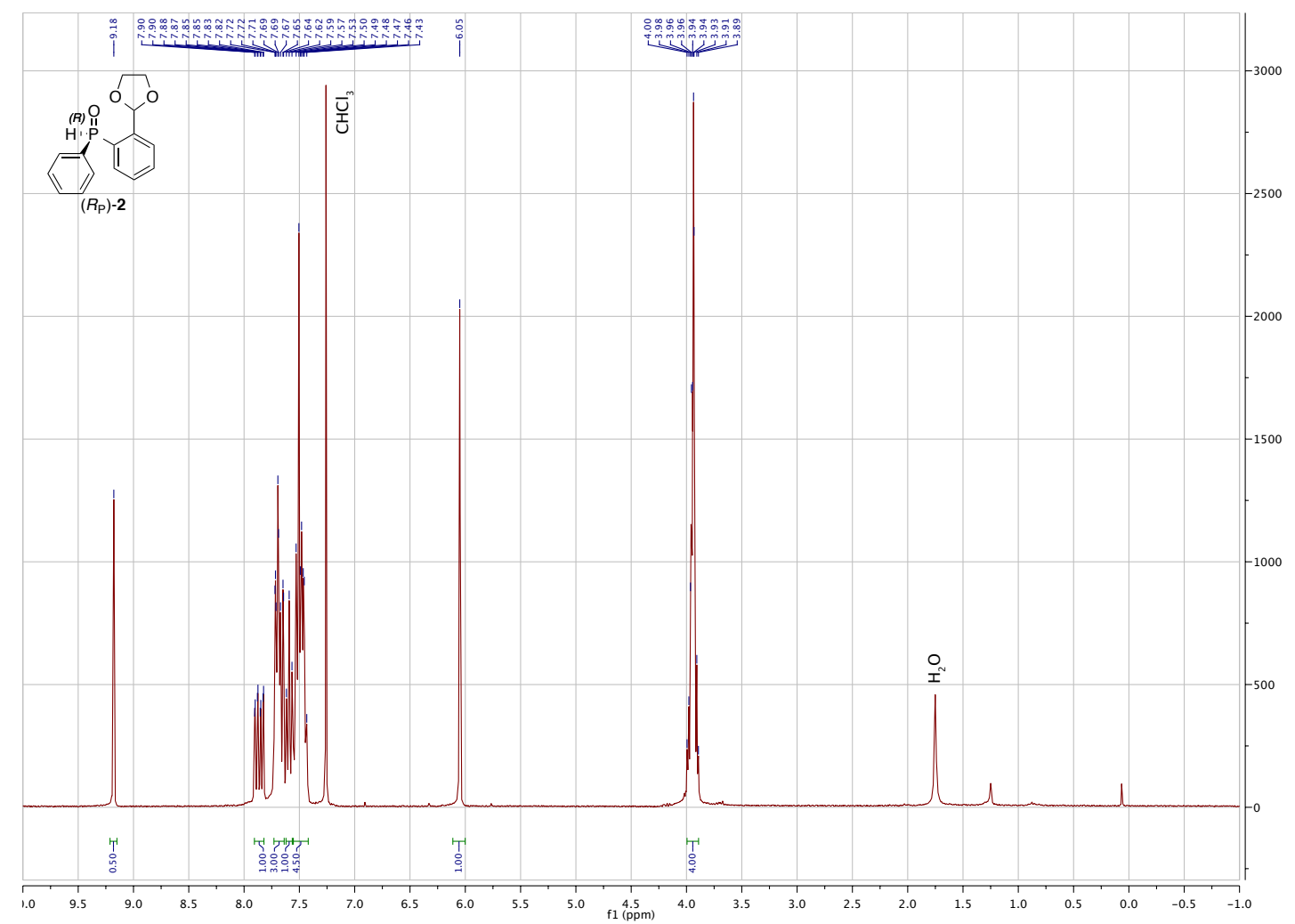

Figure S8: ${ }^{31} \mathrm{P}\left\{{ }^{1} \mathrm{H}\right\}$ NMR spectrum of $\left(R_{\mathrm{P}}\right)-2\left(122 \mathrm{MHz}, \mathrm{CDCl}_{3}\right)$.

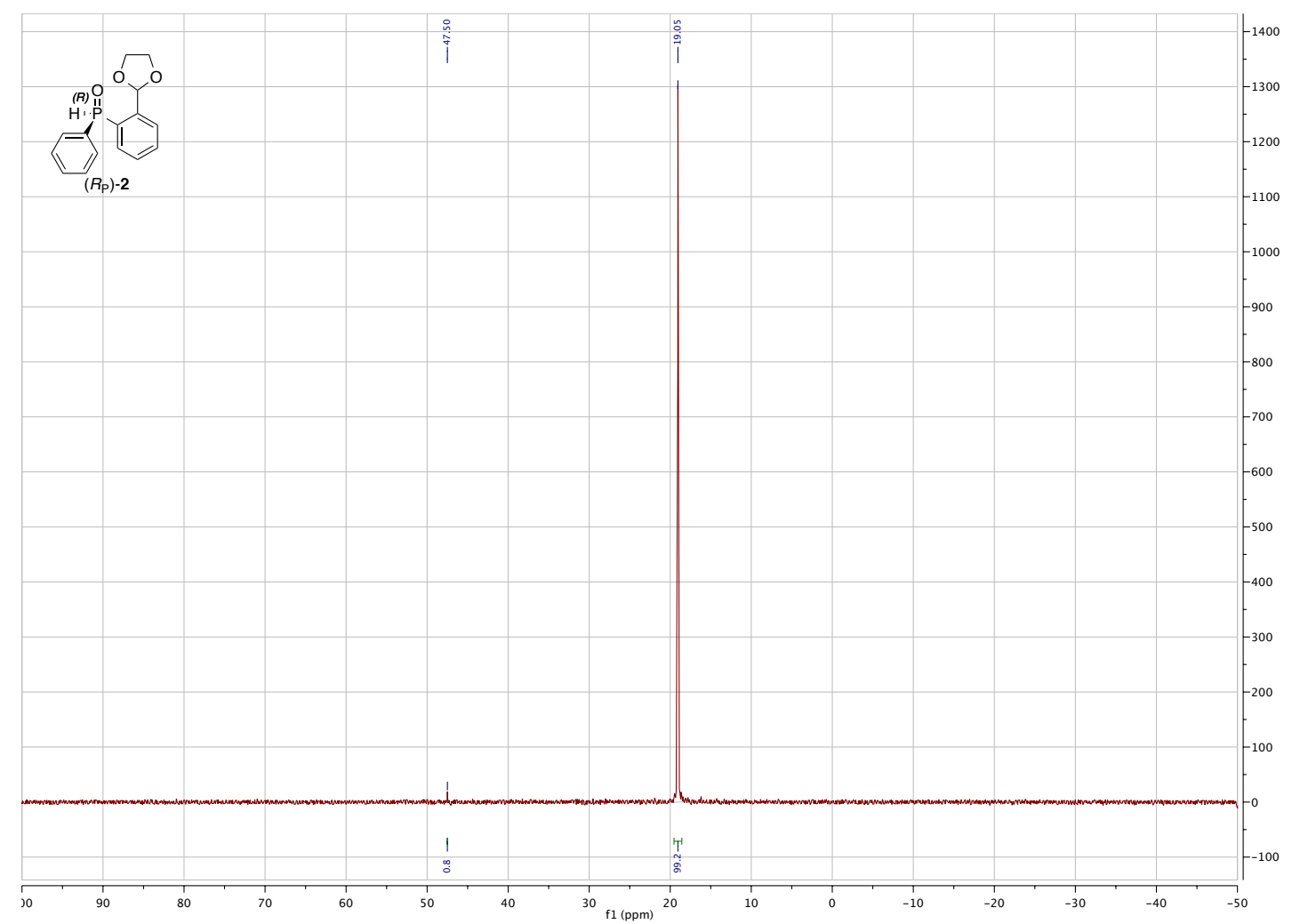


Figure S9: ${ }^{31} \mathrm{P}$ NMR spectrum of $\left(R_{\mathrm{P}}\right)-\mathbf{2}\left(122 \mathrm{MHz}, \mathrm{CDCl}_{3}\right)$.

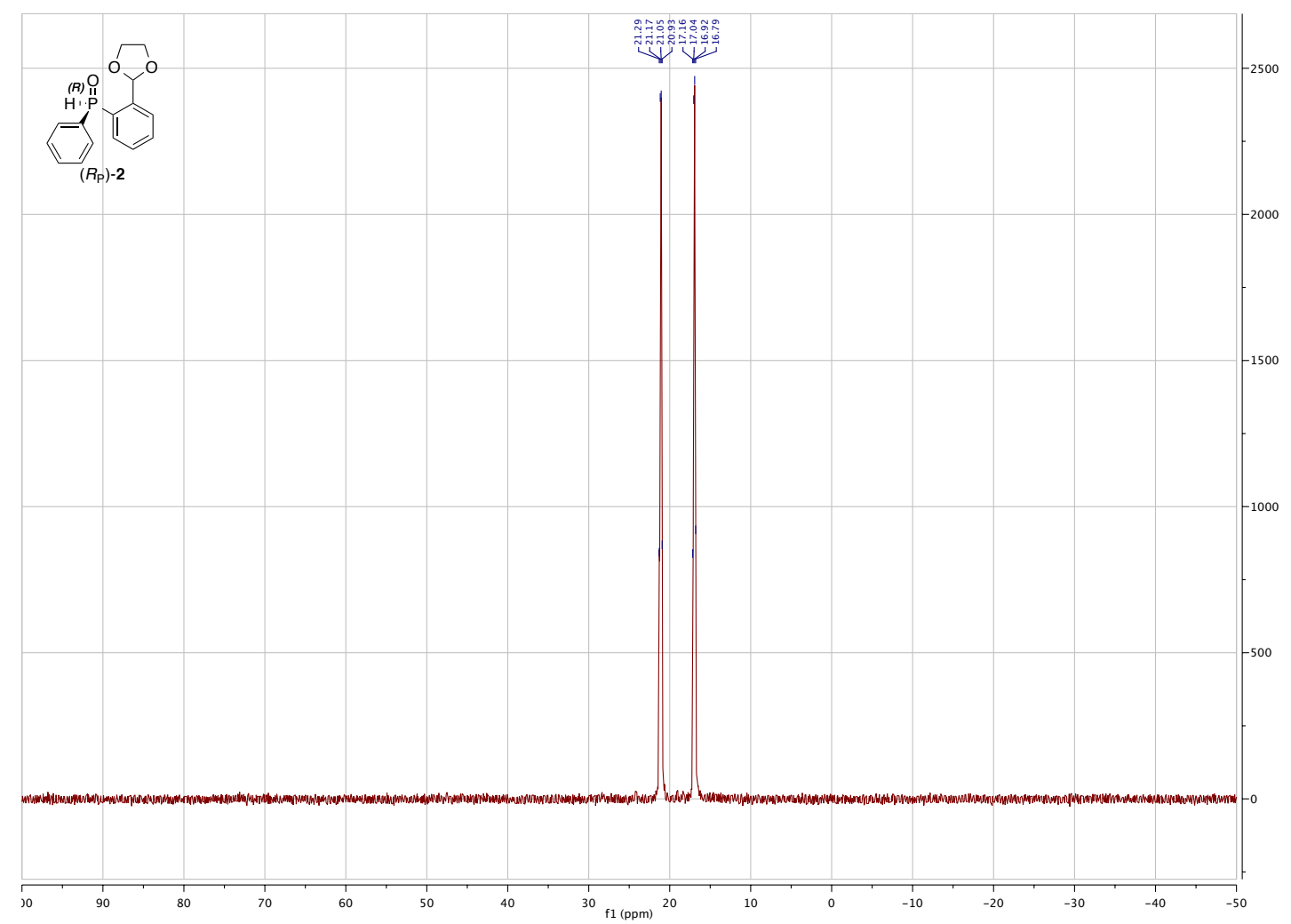

Figure S10: ${ }^{13} \mathrm{C}\left\{{ }^{1} \mathrm{H}\right\}$ NMR spectrum of $\left(R_{\mathrm{P}}\right)-2\left(75 \mathrm{MHz}, \mathrm{CDCl}_{3}\right)$.

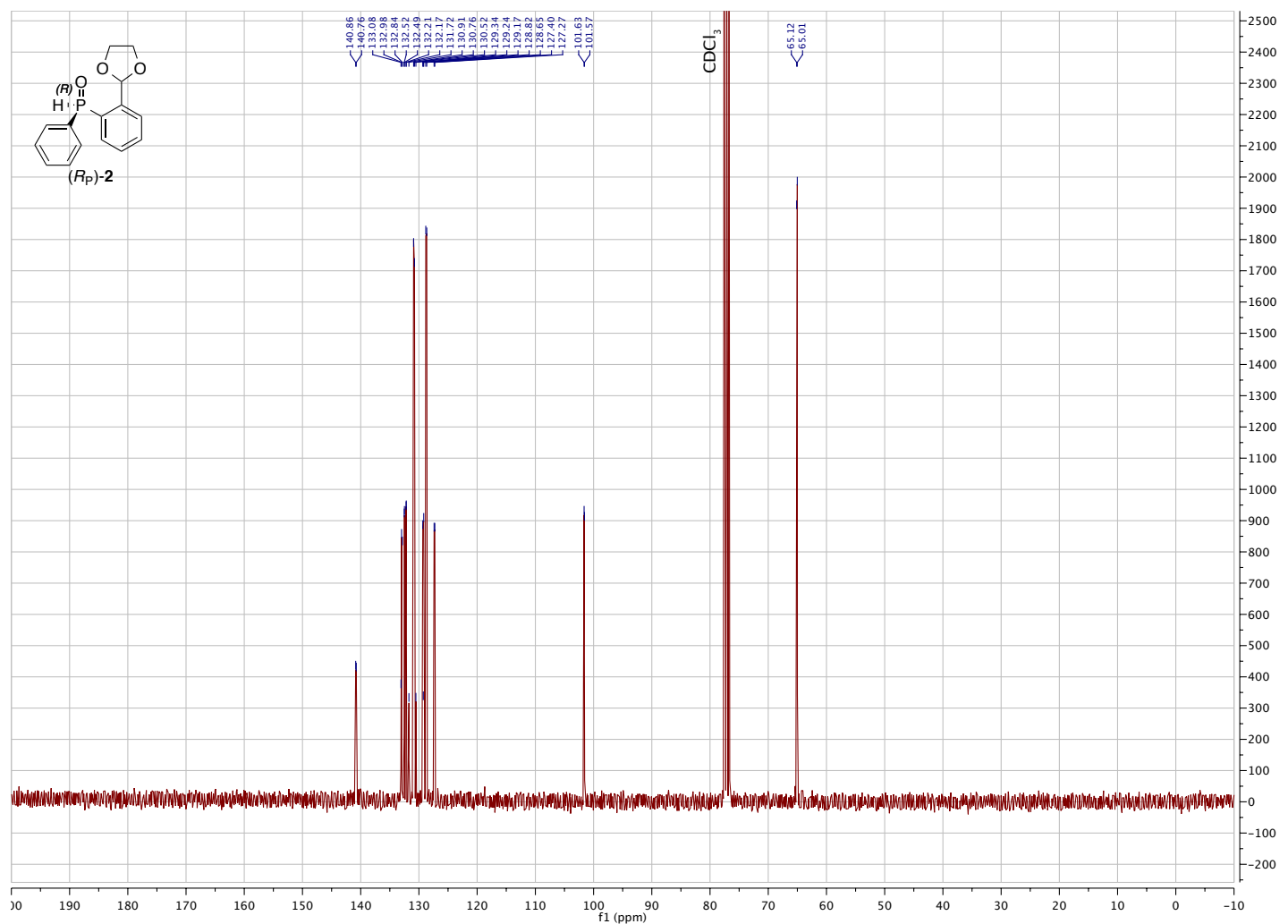


Figure S11: HPLC trace of enantioenriched $\left(R_{\mathrm{P}}\right)$-2.

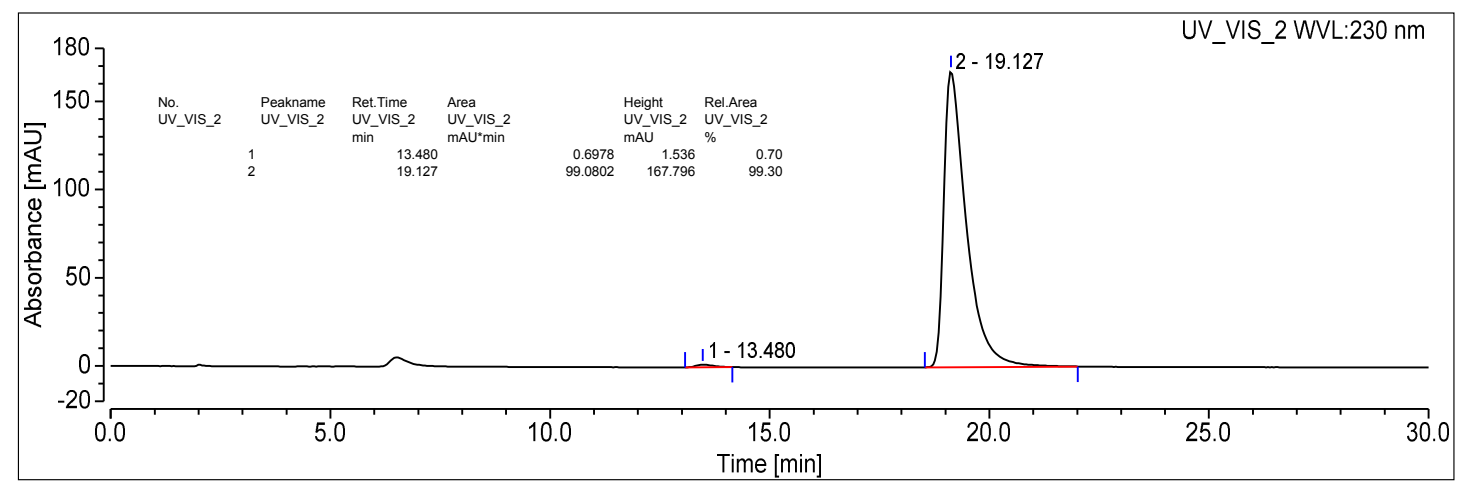

Figure S12: HPLC trace of racemic 2 (obtained from ca. 1:1 mixture of 1 (mother liquor of the first crystallization in the preparation of $\left.\left(R_{\mathrm{P}}\right)-\mathbf{1}\right)$ by the same procedure).

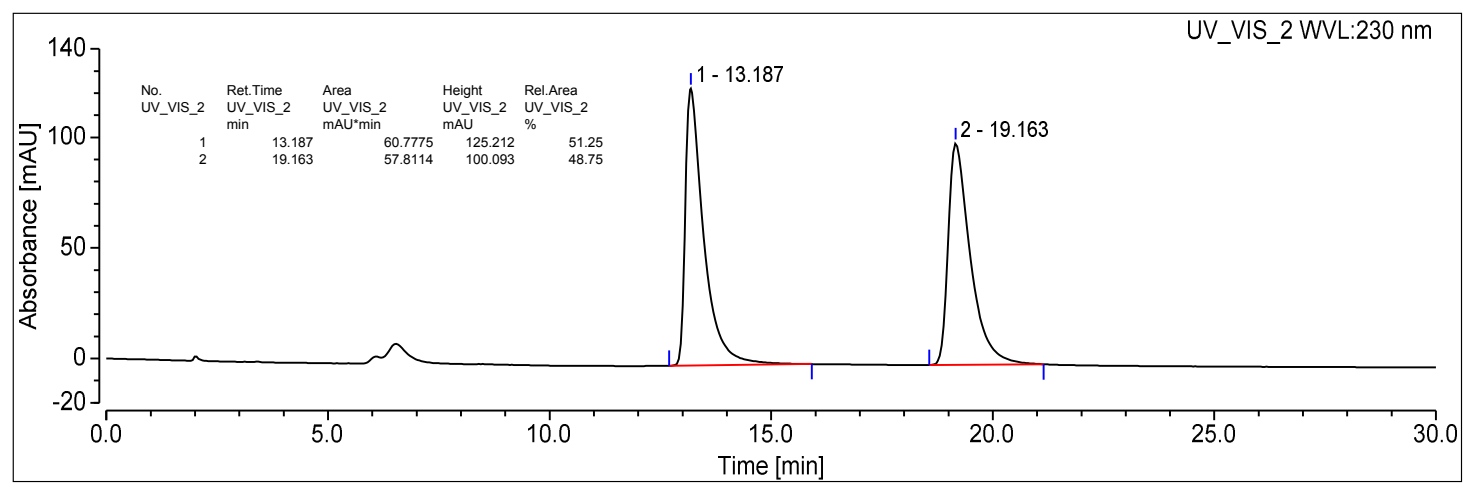

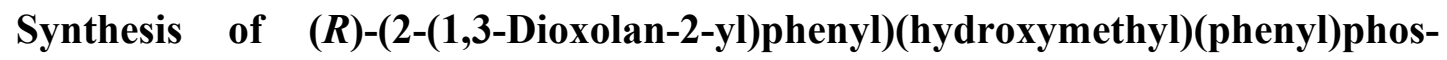

phine oxide. A $500 \mathrm{~mL}$ two-neck round-bottom flask was charged with $\left(R_{\mathrm{P}}\right)$-2 (5.99 g, $\left.21.8 \mathrm{mmol}\right)$, water $(220 \mathrm{~mL})$, potassium hydroxide $(2.45 \mathrm{~g}, 43.7 \mathrm{mmol}, 2.0$ equiv), and formaldehyde $(6.55 \mathrm{~mL}$, $37 \%$ in water, $87.4 \mathrm{mmol}, 4.0$ equiv), and the resulting solution was stirred at $60{ }^{\circ} \mathrm{C}$ for $4 \mathrm{~h}$. Saturated aqueous $\mathrm{NH}_{4} \mathrm{Cl}$ solution $(250 \mathrm{~mL})$ was added, and the solution was extracted seven times with dichloromethane $(7 \times 200 \mathrm{~mL})$. The combined organic phases were dried over $\mathrm{MgSO}_{4}$, 
filtered, and the solvent was removed under reduced pressure $\left(20 \mathrm{mbar}, 40^{\circ} \mathrm{C}\right)$. Recrystallization from hot EtOAc $(500 \mathrm{~mL})$ afforded the title compound as a clear solid. Yield: $5.24 \mathrm{~g}(79 \%$, $>99.5 \%$ ee $).{ }^{1} \mathbf{H}$ NMR $\left(300 \mathrm{MHz}, \mathrm{CDCl}_{3}\right): \delta 7.80-7.64(m, 4 \mathrm{H}, \mathrm{Ar}-H), 7.57-7.45(m, 2 \mathrm{H}, \mathrm{Ar}-H)$, $7.45-7.35(m, 3 \mathrm{H}, \mathrm{Ar}-H), 6.29\left(s, 1 \mathrm{H}, \mathrm{C} H\left(\mathrm{OCH}_{2}\right)_{2}\right), 5.62($ br $s, 1 \mathrm{H}, \mathrm{OH}), 4.55-4.40(m, 2 \mathrm{H}$, PCHHOH $), 4.00-3.74\left(m, 4 \mathrm{H}, \mathrm{CH}(\mathrm{OCHH})_{2}\right) .{ }^{31} \mathbf{P}\left\{{ }^{1} \mathbf{H}\right\}$ NMR $\left(122 \mathrm{MHz}, \mathrm{CDCl}_{3}\right): \delta 33.8(s)$. ${ }^{31}$ P NMR (122 MHz, $\left.\mathrm{CDCl}_{3}\right): \delta 33.8($ br $s) .{ }^{13} \mathbf{C}\left\{{ }^{1} \mathbf{H}\right\}$ NMR $\left(75 \mathrm{MHz}, \mathrm{CDCl}_{3}\right): \delta 142.1\left(d,{ }^{2} J_{\mathrm{P}, \mathrm{C}}=\right.$ $6.8 \mathrm{~Hz}$, arom. $), 132.9\left(d,{ }^{3} J_{\mathrm{P}, \mathrm{C}}=10.2 \mathrm{~Hz}\right.$, arom. $), 132.3\left(d,{ }^{4} J_{\mathrm{P}, \mathrm{C}}=2.6 \mathrm{~Hz}\right.$, arom. $), 132.0\left(d,{ }^{4} J_{\mathrm{P}, \mathrm{C}}=\right.$ $2.7 \mathrm{~Hz}$, arom. $), 131.8\left(d,{ }^{1} J_{\mathrm{P}, \mathrm{C}}=97.2 \mathrm{~Hz}\right.$, arom. $), 131.3\left(d,{ }^{3} J_{\mathrm{P}, \mathrm{C}}=9.4 \mathrm{~Hz}\right.$, arom. $), 129.2\left(d,{ }^{1} J_{\mathrm{P}, \mathrm{C}}=\right.$ $90.8 \mathrm{~Hz}$, arom. $), 128.9\left(d,{ }^{2} J_{\mathrm{P}, \mathrm{C}}=11.6 \mathrm{~Hz}\right.$, arom. $), 128.6\left(d,{ }^{2} J_{\mathrm{P}, \mathrm{C}}=11.7 \mathrm{~Hz}\right.$, arom. $), 127.4\left(d,{ }^{3} J_{\mathrm{P}, \mathrm{C}}=\right.$ $9.0 \mathrm{~Hz}$, arom. $), 100.3\left(d,{ }^{3} \mathrm{P}_{\mathrm{P}, \mathrm{C}}=4.0 \mathrm{~Hz}, \mathrm{CH}\left(\mathrm{OCH}_{2}\right)_{2}\right), 65.23\left(\mathrm{CH}\left(\mathrm{OCH}_{2}\right)_{2}\right), 65.21\left(\mathrm{CH}\left(\mathrm{OCH}_{2}\right)_{2}\right)$, $62.1\left(d,{ }^{1} J_{\mathrm{P}, \mathrm{C}}=82.1 \mathrm{~Hz}, \mathrm{PCH}_{2} \mathrm{OH}\right)$. Melting Point: $170.0^{\circ} \mathrm{C}$. IR (liquid film, $\left.\mathrm{cm}^{-1}\right): 3214(\mathrm{O}-\mathrm{H})$, 3061 (C-H), 2955 (C-H), $2885(\mathrm{C}-\mathrm{H}), 2245(\mathrm{P}=\mathrm{O}), 1591,1572,1483,1473,1437,1395,1203$, 1158, 1133, 1111, 1092, 1058, 1027. HRMS (ESI): Calcd. for $\mathrm{C}_{16} \mathrm{H}_{18} \mathrm{O}_{4} \mathrm{P} m / z=305.0937$, found $m / z=305.0935[\mathrm{M}+\mathrm{H}]^{+}$. EA: Calcd. for $\mathrm{C}_{16} \mathrm{H}_{17} \mathrm{O}_{4} \mathrm{P}: \mathrm{C}, 63.16 ; \mathrm{H}, 5.63$; found: $\mathrm{C}, 62.94 ; \mathrm{H}, 5.58$. HPLC: Chiralpak ID-3 (hexane : $2-\mathrm{PrOH}=80: 20$, flow rate $1.0 \mathrm{~mL} / \mathrm{min}, \lambda=210 \mathrm{~nm}$ ), retention times $t_{\mathrm{R}}($ major $)=22.5 \mathrm{~min}, t_{\mathrm{R}}($ minor $)=31.4 \mathrm{~min} ;>99.5 \% e e .[\alpha]_{\mathbf{D}}{ }^{20}:-3.1\left(\mathrm{c}=1.0, \mathrm{CH}_{2} \mathrm{Cl}_{2}\right)$. 
Figure S13: ${ }^{1} \mathrm{H}$ NMR spectrum of the hydroxymethyl derivative of $\left(R_{\mathrm{P}}\right)-2\left(300 \mathrm{MHz}, \mathrm{CDCl}_{3}\right)$.

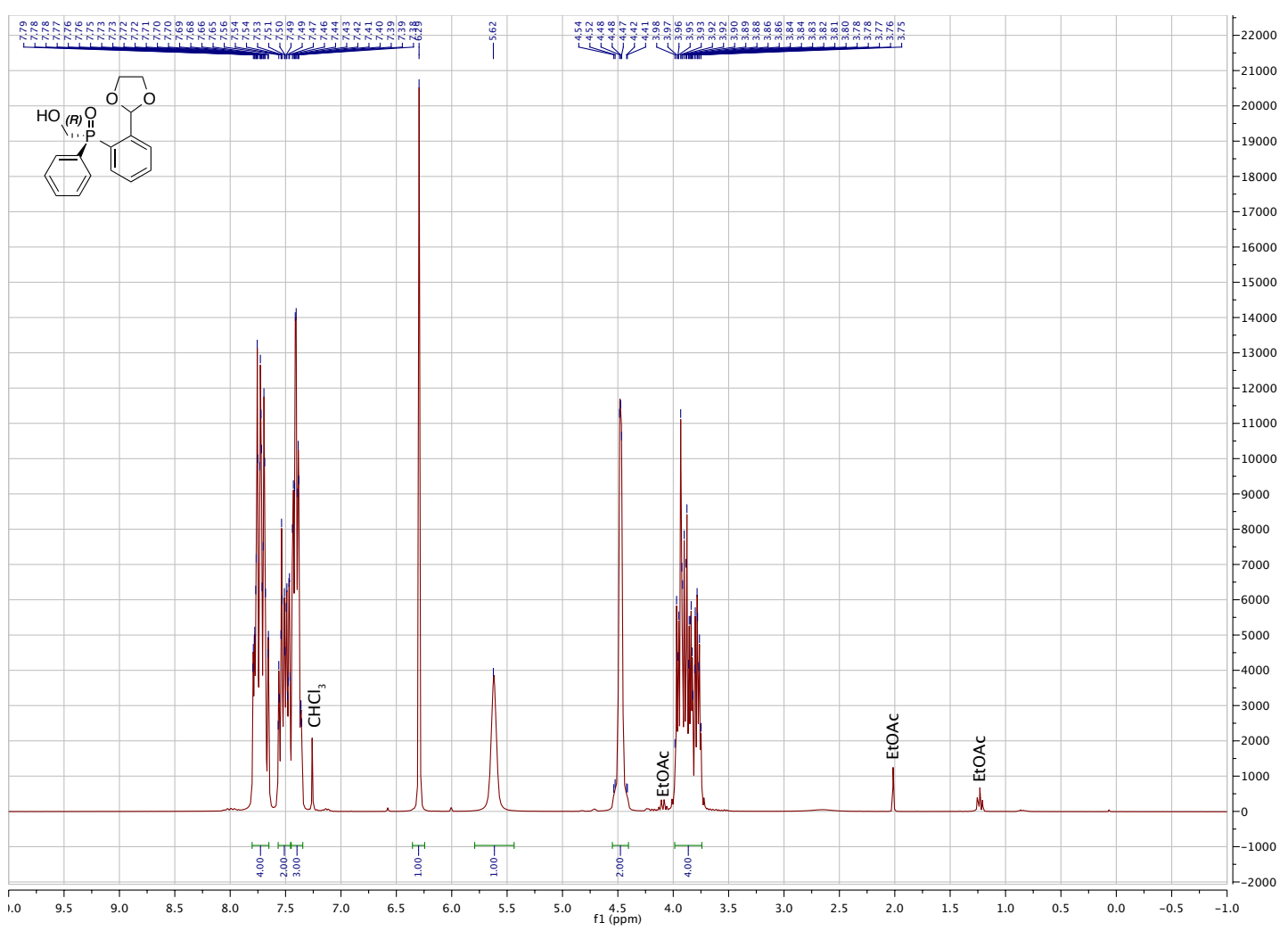

Figure S14: ${ }^{31} \mathrm{P}\left\{{ }^{1} \mathrm{H}\right\}$ NMR spectrum of the hydroxymethyl derivative of $\left(R_{\mathrm{P}}\right)-2\left(122 \mathrm{MHz}, \mathrm{CDCl}_{3}\right)$.

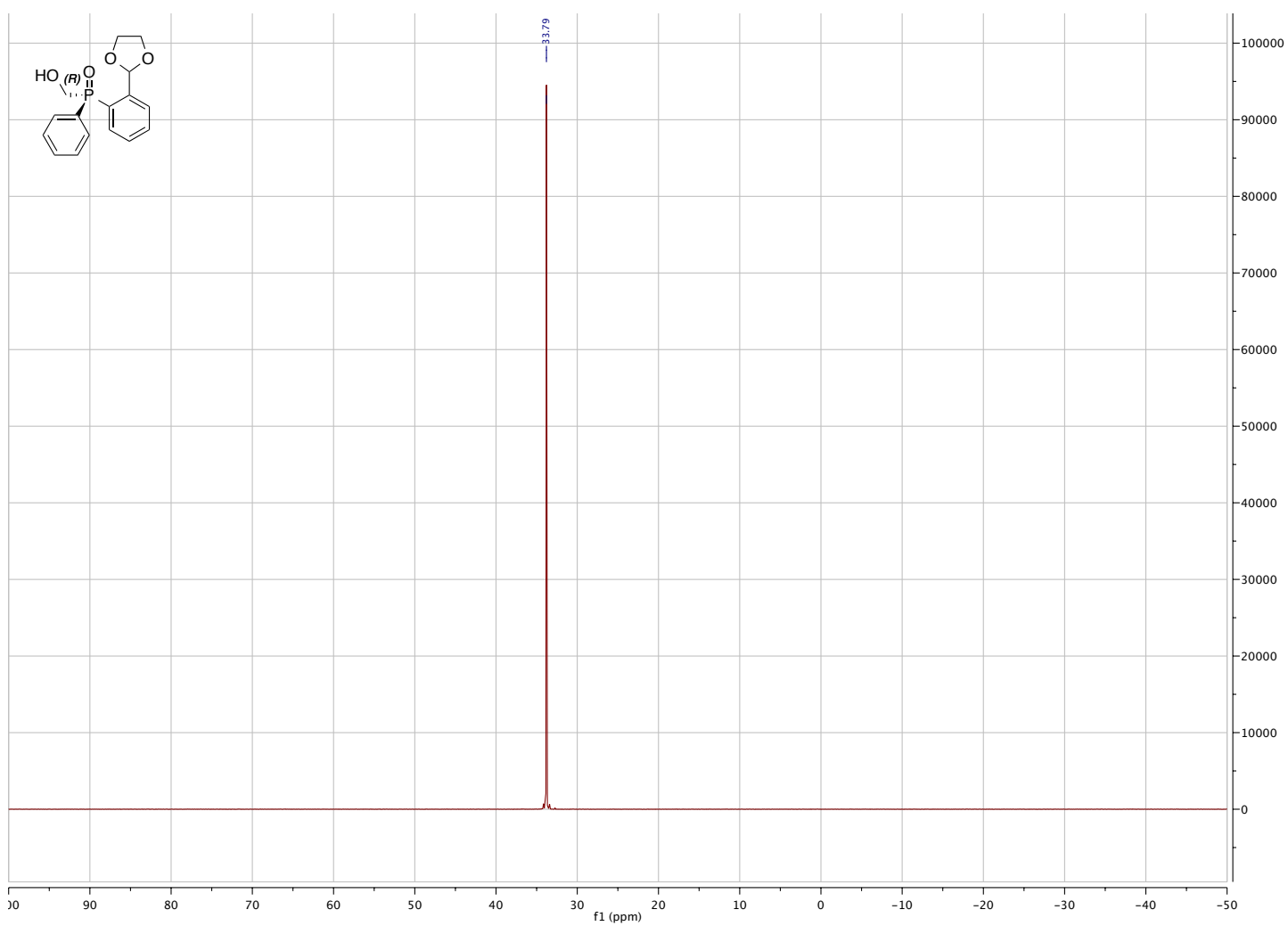


Figure S15: ${ }^{31} \mathrm{P}$ NMR spectrum of the hydroxymethyl derivative of $\left(R_{\mathrm{P}}\right)-2\left(122 \mathrm{MHz}, \mathrm{CDCl}_{3}\right)$.

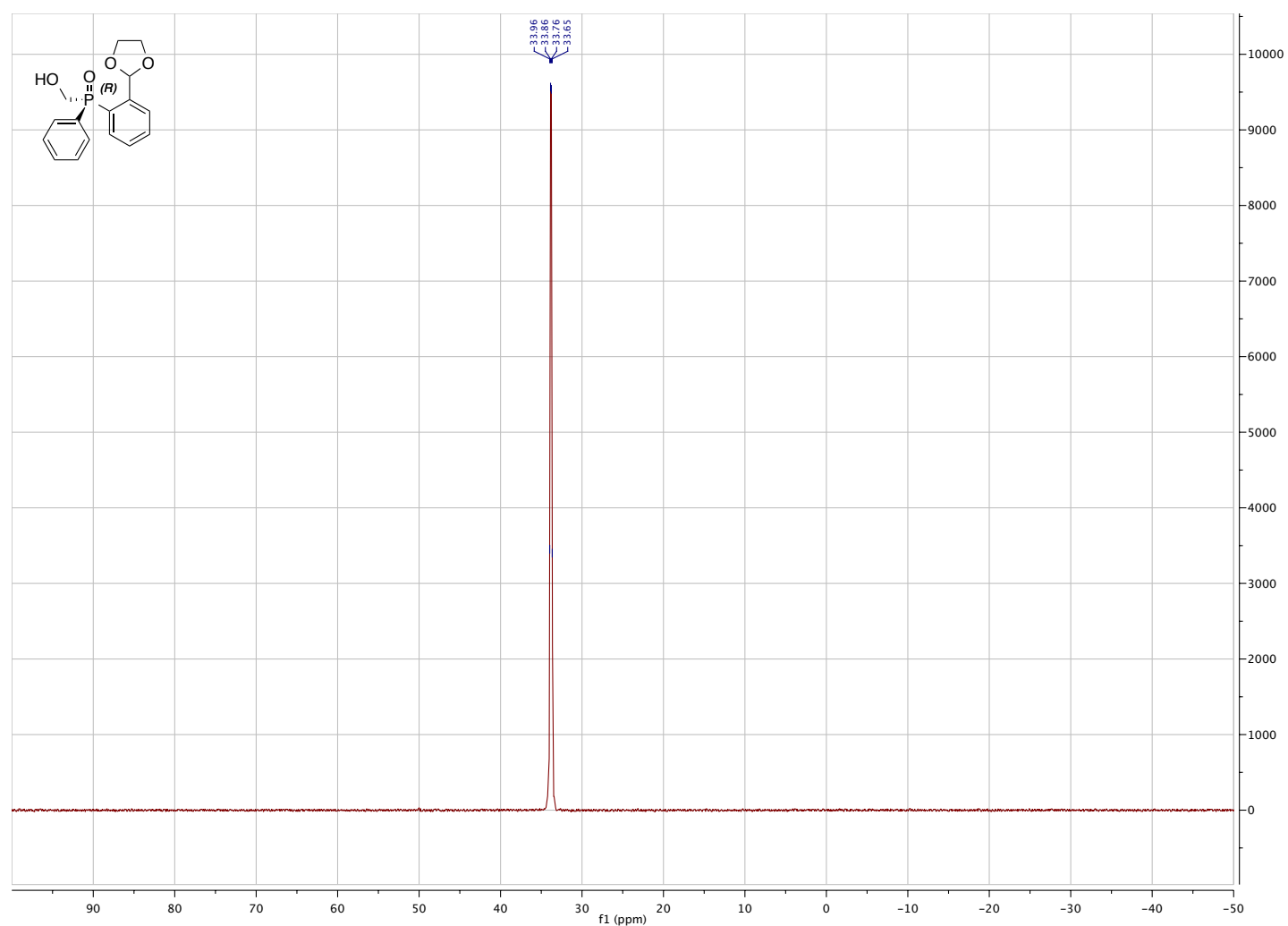

Figure S16: ${ }^{13} \mathrm{C}\left\{{ }^{1} \mathrm{H}\right\}$ NMR spectrum of the hydroxymethyl derivative of $\left(R_{\mathrm{P}}\right)-2\left(75 \mathrm{MHz}, \mathrm{CDCl}_{3}\right)$.
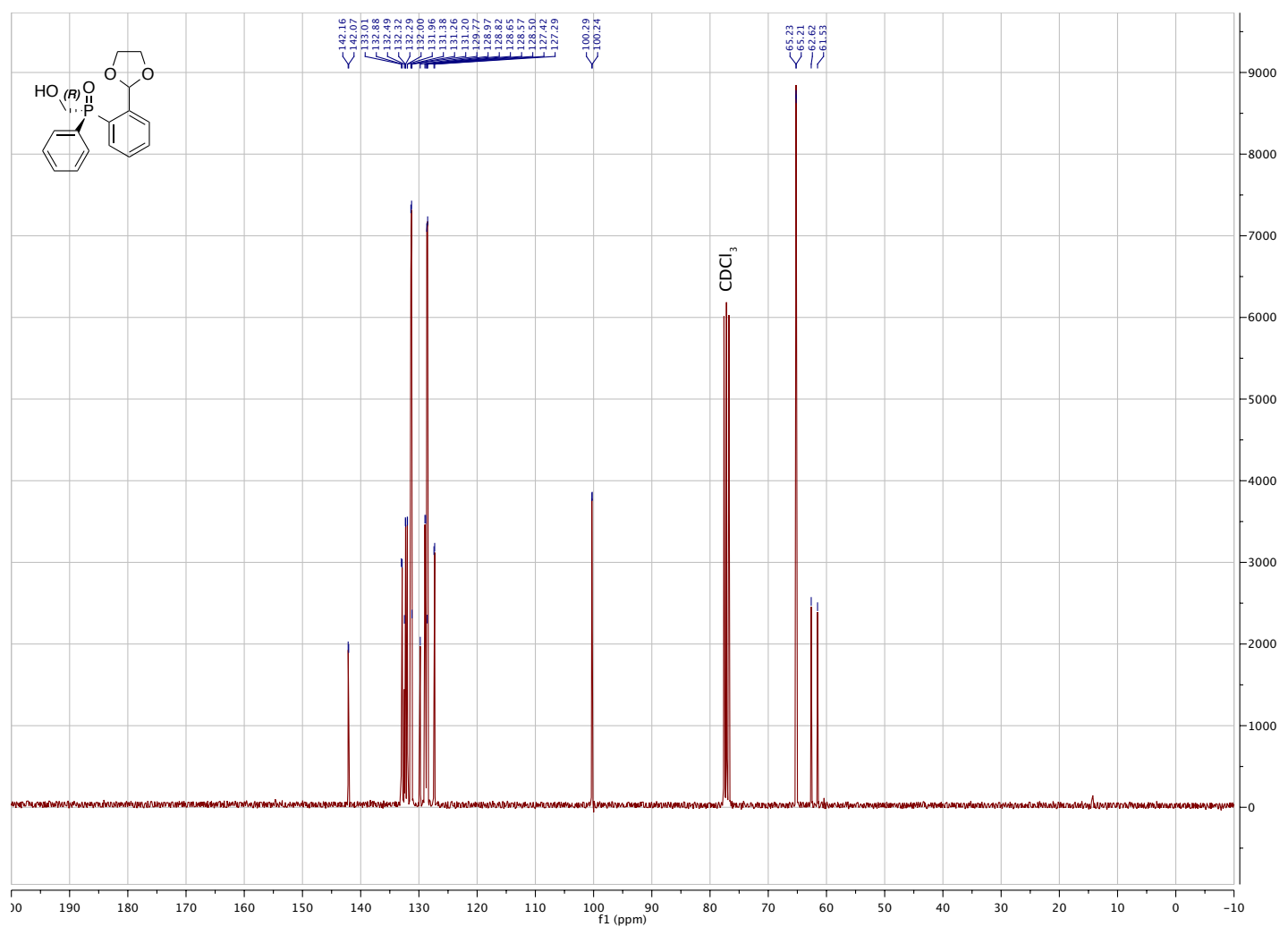
Figure S17: HPLC trace of the hydroxymethyl derivative of $\left(R_{\mathrm{P}}\right)$-2.

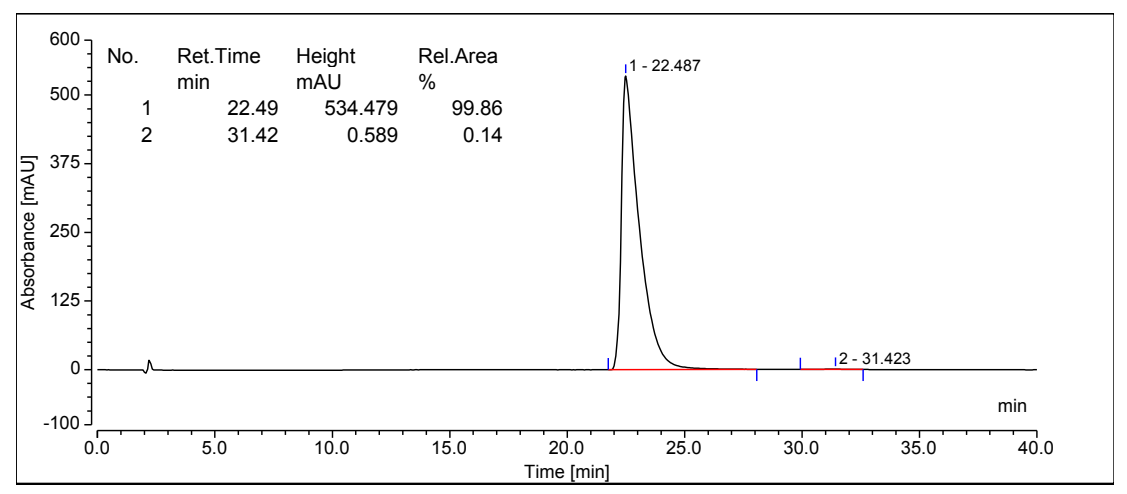

Figure S18: HPLC trace of the hydroxymethyl derivative of ( $\mathrm{rac})-2$.

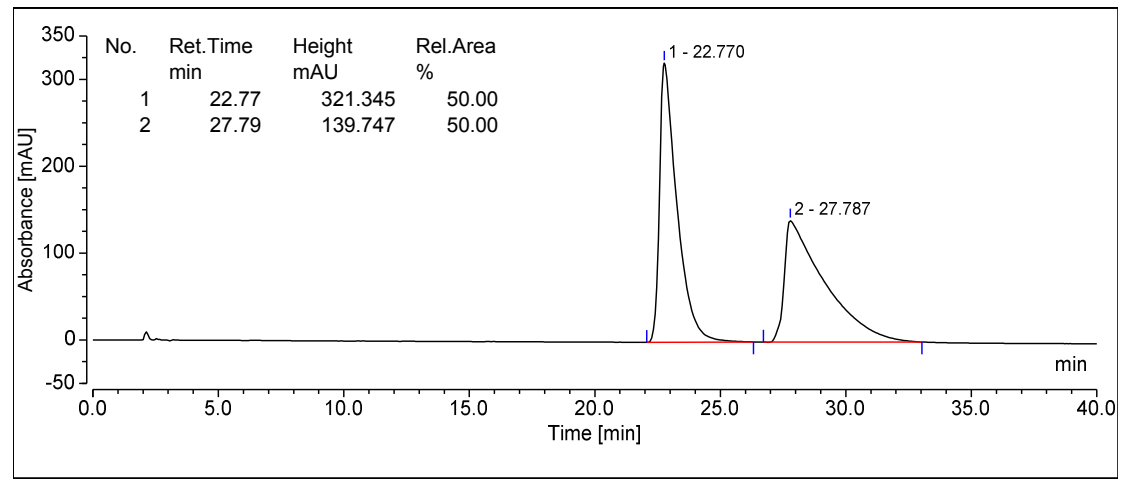

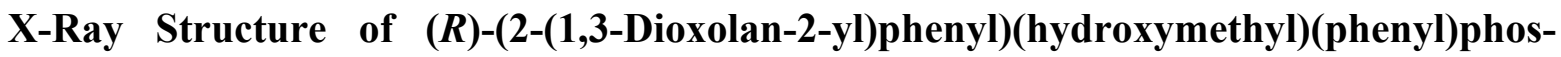

phine oxide. Colorless crystals of $(R)-(2-(1,3-$ dioxolan-2-yl)phenyl)(hydroxymethyl)(phenyl)phosphine oxide were obtained by crystallization from hot EtOAc. Crystal data for $\mathrm{C}_{16} \mathrm{H}_{17} \mathrm{O}_{4} \mathrm{P}$ : block $(0.44 \times 0.36 \times 0.29 \mathrm{~mm})$, monoclinic, $P 2_{1}$, cell dimensions $(100 \mathrm{~K}) a=8.4621(4), b=$ $10.2910(6), c=8.8558(6) \AA, \beta=105.314(6)^{\circ}$ and $V=743.81(8) \AA^{3}$ with $Z=2, D_{\mathrm{c}}=1.359 \mathrm{Mg} / \mathrm{m}^{3}$, $\mu=0.198 \mathrm{~mm}^{-1}$ (Mo $\mathrm{K}_{\alpha}$, graphite monochromated), $\lambda=0.71073 \AA, F(000)=320$. The data were collected at $100 \mathrm{~K}$ on a Bruker AXS SMART APEX2 platform in the $\theta$ range $2.96-29.56^{\circ}$. The structure was solved with SHELXS using direct methods. Of the 6'869 measured reflections with index ranges $-11 \leq h \leq 11,-14 \leq k \leq 13,-12 \leq l \leq 12,3^{\prime}$ '877 independent reflections $\left(R_{\text {int }}=0.048\right)$ were used in the refinement (full-matrix least squares on $F^{2}$ ) with anisotropic displacement 
parameters for all non-H atoms. Hydrogen atoms were introduced at calculated positions and refined with the riding model and individual isotropic thermal parameters for each group. The asymmetric unit contains one molecule of (R)-(2-(1,3-dioxolan-2-yl)phenyl)(hydroxymethyl)(phenyl)phosphine oxide. Final residuals were $R_{1}=0.0413$ (for 3'517 reflections with $I>2 \sigma(I)$ ) and $\mathrm{w} R_{2}=0.0927$ (all data), GOF $=1.030$, absolute structure parameter 0.05(7). Max. and min. difference peaks were +0.29 and $-0.26 \mathrm{e}^{-3}$, the largest and mean $\Delta / \sigma=0.000$ and 0.000 .

Figure S19: X-ray structure of the hydroxymethyl derivative of $\left(R_{\mathrm{P}}\right)-\mathbf{2}$.
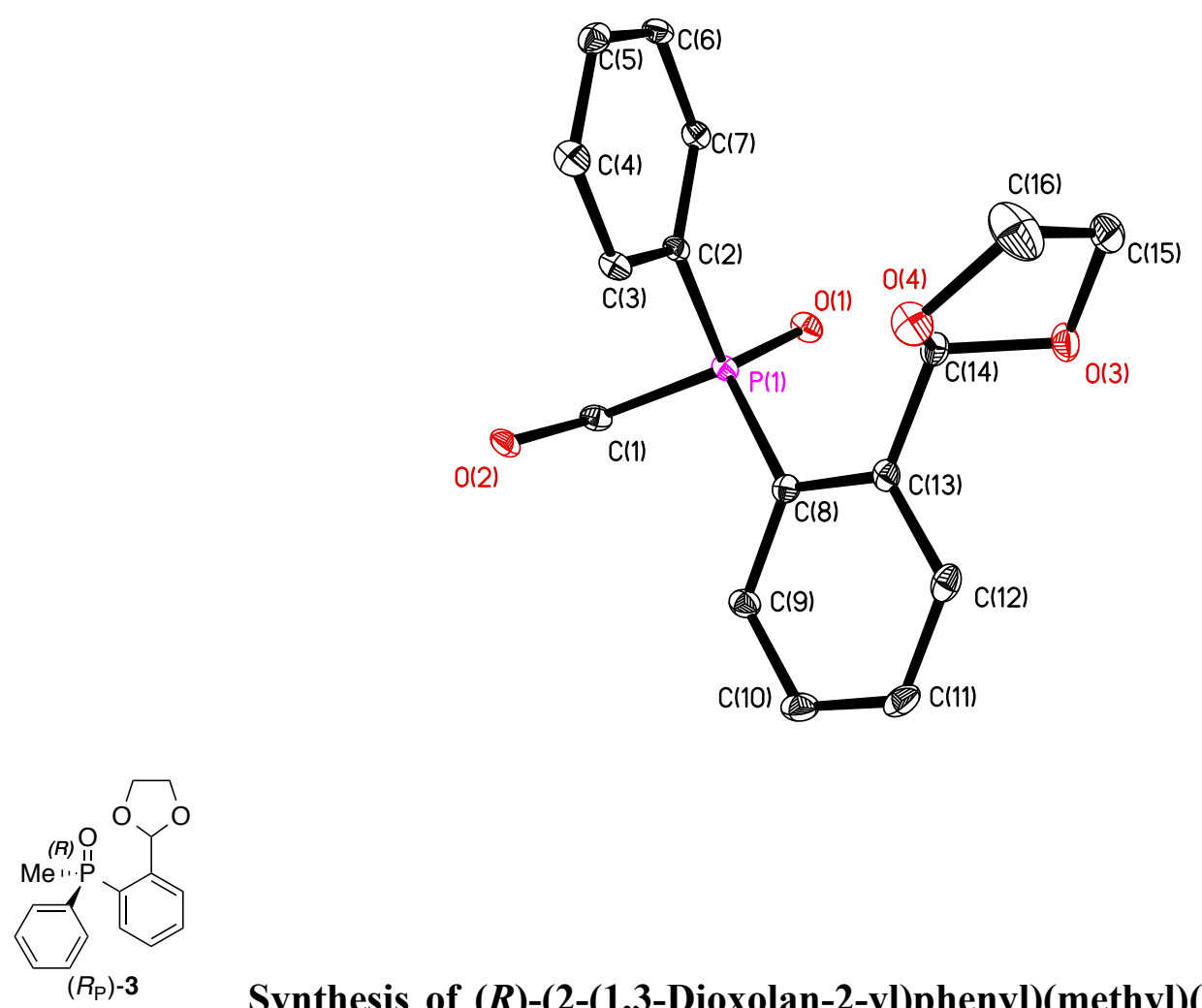

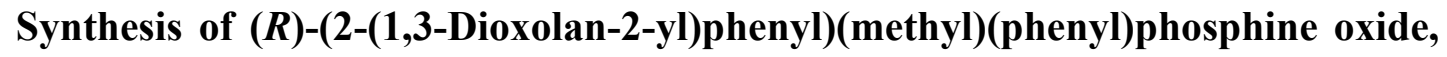

$\left(\boldsymbol{R}_{\mathbf{P}}\right)$-3. A flame-dried $1000 \mathrm{~mL}$ three-neck round-bottom flask connected to a $100 \mathrm{~mL}$ Young addition funnel was charged with $\left(R_{\mathrm{P}}\right)-\mathbf{2}(22.1 \mathrm{~g}, 81 \mathrm{mmol})$ and THF $(410 \mathrm{~mL})$, and the resulting solution was cooled to $-78^{\circ} \mathrm{C}$. After $10 \mathrm{~min}, n$-butyllithium $(50.4 \mathrm{~mL}, 1.6 \mathrm{M}$ in hexanes, $81 \mathrm{mmol}$, 1.0 equiv) was added, and the solution was stirred for $30 \mathrm{~min}$ at $-78{ }^{\circ} \mathrm{C}$ (Note: A color change from light orange to dark red indicates full deprotonation of the secondary phosphine oxide). 
Iodomethane (5.5 mL, $89 \mathrm{mmol}, 1.1$ equiv) was dissolved in THF (46 mL) and added over $1 \mathrm{~h}$ using the addition funnel. The solution was warmed to room temperature over $3 \mathrm{~h}$, and the solvent was removed at the rotary evaporator $\left(20 \mathrm{mbar}, 40^{\circ} \mathrm{C}\right)$. Chloroform $(250 \mathrm{~mL})$ and water $(250 \mathrm{~mL})$ were added, and the mixture was vigorously stirred for $15 \mathrm{~min}$ at room temperature. The organic phase was separated, and the aqueous phase was extracted twice with chloroform $(2 \times 250 \mathrm{~mL})$. The combined organic phases were dried over $\mathrm{MgSO}_{4}$, filtered, and the solvent was removed at the rotary evaporator $\left(20 \mathrm{mbar}, 40{ }^{\circ} \mathrm{C}\right)$ to afford the title compound $\left(>96 \%\right.$ pure by $\left.{ }^{31} \mathrm{P}\left\{{ }^{1} \mathrm{H}\right\} \mathrm{NMR}\right)$ as a clear oil that solidified upon standing (Notes: If the crude was $<95 \%$ pure by ${ }^{31} \mathrm{P}\left\{{ }^{1} \mathrm{H}\right\} \mathrm{NMR}$, flash column chromatography on silica gel (gradient $\mathrm{CH}_{2} \mathrm{Cl}_{2}: \mathrm{MeOH}=100: 0$ to $94: 6$ ) gave pure product; if ee was below $95 \%$, recrystallization from THF at $-18{ }^{\circ} \mathrm{C}$ and removal of the precipitated racemate by filtration afforded the product with $\geq 97 \% e e)$. Yield: $22.0 \mathrm{~g}(95 \%, 97 \%$ ee $) .{ }^{1} \mathbf{H}$ NMR $\left(300 \mathrm{MHz}, \mathrm{CDCl}_{3}\right): \delta 7.83-7.78(m, 1 \mathrm{H}, \mathrm{Ar}-H), 7.75-7.66(m, 2 \mathrm{H}, \mathrm{Ar}-H), 7.62-7.37(m, 6 \mathrm{H}$, $\operatorname{Ar}-H), 6.41\left(s, 1 \mathrm{H}, \mathrm{CH}\left(\mathrm{OCH}_{2}\right)_{2}\right), 4.09-3.85\left(m, 4 \mathrm{H}, \mathrm{CH}(\mathrm{OCHH})_{2}\right), 2.11\left(d,{ }^{2} J_{\mathrm{P}, \mathrm{H}}=13.3 \mathrm{~Hz}, 3 \mathrm{H}\right.$, $\left.\mathrm{PCH}_{3}\right) .{ }^{31} \mathbf{P}\left\{{ }^{1} \mathbf{H}\right\}$ NMR (122 MHz, $\left.\mathrm{CDCl}_{3}\right): \delta 27.0(s) .{ }^{31} \mathbf{P}$ NMR $\left(122 \mathrm{MHz}, \mathrm{CDCl}_{3}\right): \delta 27.0(q d$, $\left.{ }^{2} J_{\mathrm{P}, \mathrm{H}}=13.3 \mathrm{~Hz},{ }^{3} J_{\mathrm{P}, \mathrm{H}}=11.6 \mathrm{~Hz}\right) .{ }^{13} \mathbf{C}\left\{{ }^{1} \mathbf{H}\right\} \mathbf{N M R}\left(75 \mathrm{MHz}, \mathrm{CDCl}_{3}\right): \delta 141.7\left(d,{ }^{2} J_{\mathrm{P}, \mathrm{C}}=7.3 \mathrm{~Hz}\right.$, arom.), 134.8 (d, ${ }^{1} J_{\mathrm{P}, \mathrm{C}}=101.8 \mathrm{~Hz}$, arom.), 132.5 (d, ${ }^{1} J_{\mathrm{P}, \mathrm{C}}=89.6 \mathrm{~Hz}$, arom.), $132.1\left(d,{ }^{4} J_{\mathrm{P}, \mathrm{C}}=2.8 \mathrm{~Hz}\right.$, arom.), $132.0\left(d,{ }^{2} J_{\mathrm{P}, \mathrm{C}}=10.8 \mathrm{~Hz}\right.$, arom.), $131.8\left(d,{ }^{4} J_{\mathrm{P}, \mathrm{C}}=2.7 \mathrm{~Hz}\right.$, arom.), $130.7\left(d,{ }^{3} J_{\mathrm{P}, \mathrm{C}}=9.8 \mathrm{~Hz}\right.$, arom.), 128.9 (d, ${ }^{3} J_{\mathrm{P}, \mathrm{C}}=7.3 \mathrm{~Hz}$, arom.), $128.7\left(d,{ }^{2} J_{\mathrm{P}, \mathrm{C}}=12.0 \mathrm{~Hz}\right.$, arom. $), 127.5\left(d,{ }^{3} J_{\mathrm{P}, \mathrm{C}}=9.5 \mathrm{~Hz}\right.$, arom.), $100.2\left(d,{ }^{3} J_{\mathrm{P}, \mathrm{C}}=4.4 \mathrm{~Hz}, \mathrm{CH}\left(\mathrm{OCH}_{2}\right)_{2}\right), 65.4\left(2 \mathrm{C}, \mathrm{CH}\left(\mathrm{OCH}_{2}\right)_{2}\right), 18.2\left(d,{ }^{1} J_{\mathrm{P}, \mathrm{C}}=74.2 \mathrm{~Hz}\right.$, $\mathrm{CH}_{3}$ ). Melting Point: $108{ }^{\circ} \mathrm{C}$. IR (liquid film, $\left.\mathrm{cm}^{-1}\right)$ : $3058(\mathrm{C}-\mathrm{H}), 2956(\mathrm{C}-\mathrm{H}), 2887$ (C-H), $2216(\mathrm{P}=\mathrm{O}), 1643,1591,1572,1473,1437,1395,1296,1177,1132,1113,1091,1060,1025$. EA: Calcd. for $\mathrm{C}_{16} \mathrm{H}_{17} \mathrm{O}_{3} \mathrm{P}: \mathrm{C}$, 66.66; H, 5.94; found: C, 66.48; H, 6.04. HRMS (ESI): Calcd. for $\mathrm{C}_{16} \mathrm{H}_{18} \mathrm{O}_{3} \mathrm{P} m / z=289.0988$, found $m / z=289.0985[\mathrm{M}+\mathrm{H}]^{+}$. HPLC: Chiralpak ID-3 (hexane : $2-\mathrm{PrOH}=90: 10$, flow rate $1.0 \mathrm{~mL} / \mathrm{min}, \lambda=210 \mathrm{~nm}$ ), retention times $t_{\mathrm{R}}$ (major) $=75.0 \mathrm{~min}$, $t_{\mathrm{R}}($ minor $)=82.3 \mathrm{~min} ; 97 \%$ ee. $[\boldsymbol{\alpha}]_{\mathbf{D}}{ }^{20}:-16.9\left(\mathrm{c}=1.0, \mathrm{CH}_{2} \mathrm{Cl}_{2}\right)$. 
Figure S20: ${ }^{1} \mathrm{H}$ NMR spectrum of $\left(R_{\mathrm{P}}\right)$-3 $\left(300 \mathrm{MHz}, \mathrm{CDCl}_{3}\right)$.

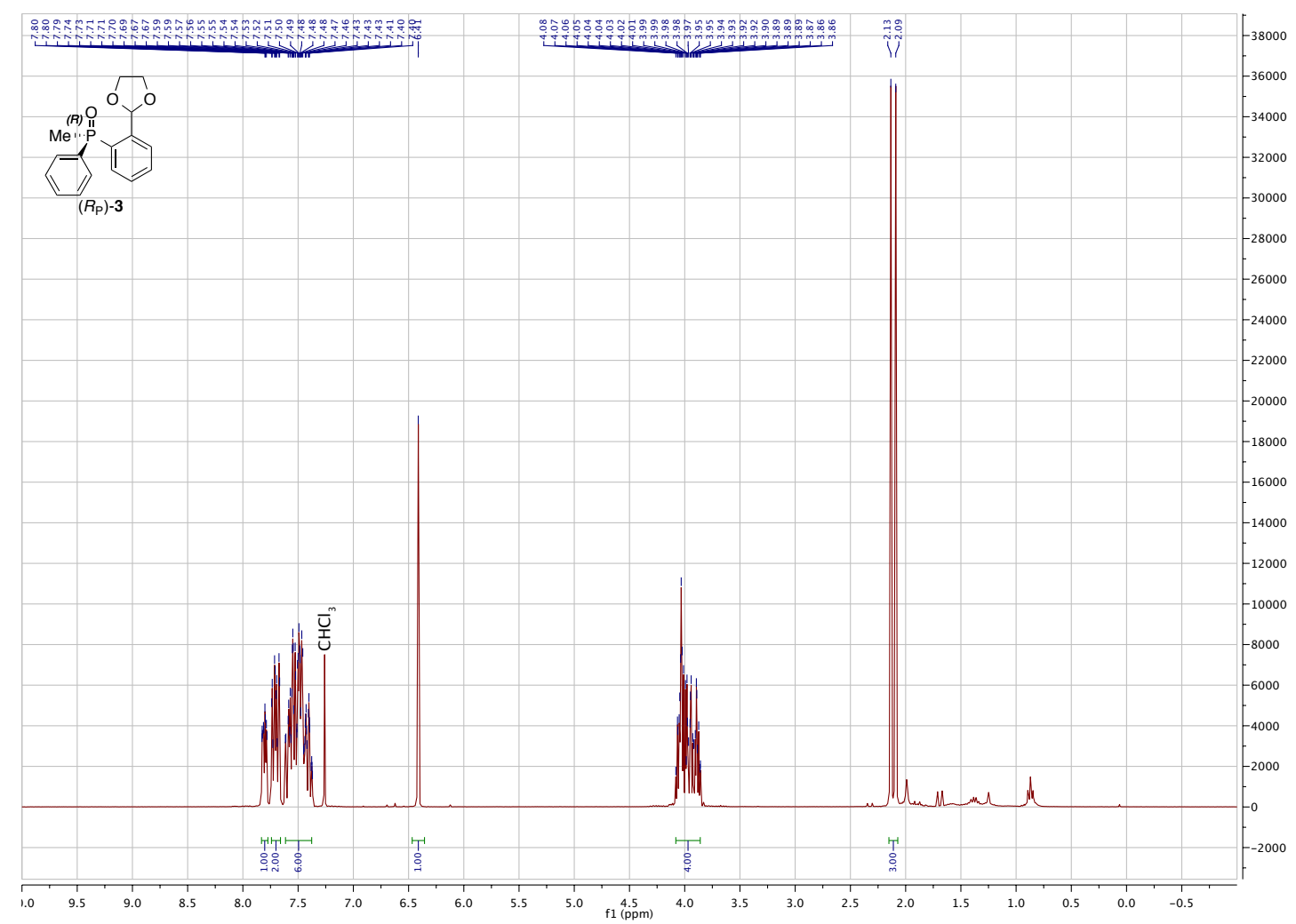

Figure S21: ${ }^{31} \mathrm{P}\left\{{ }^{1} \mathrm{H}\right\}$ NMR spectrum of $\left(R_{\mathrm{P}}\right)-\mathbf{3}\left(122 \mathrm{MHz}, \mathrm{CDCl}_{3}\right)$.

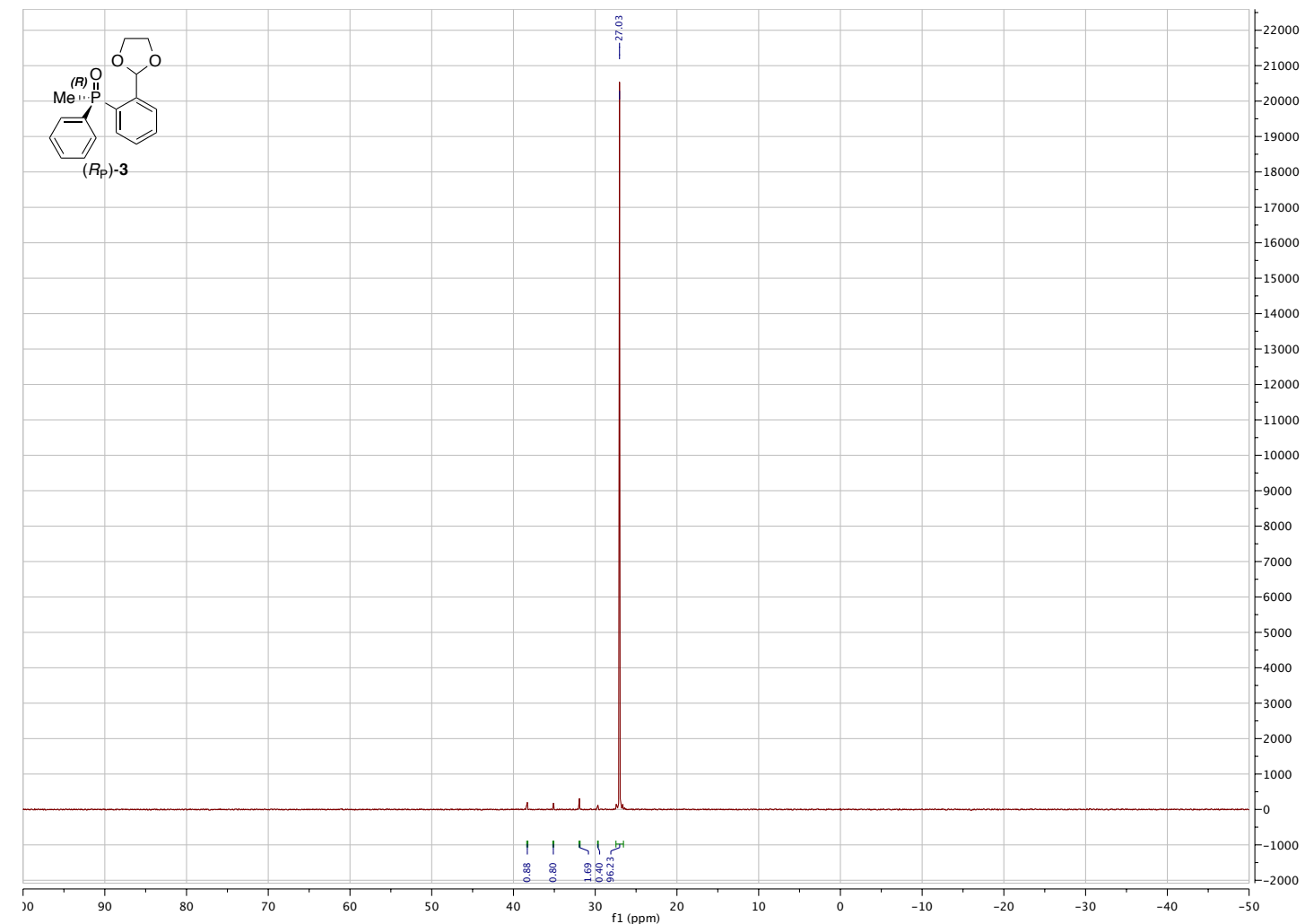


Figure S22: ${ }^{31} \mathrm{P}$ NMR spectrum of $\left(R_{\mathrm{P}}\right)-3\left(122 \mathrm{MHz}, \mathrm{CDCl}_{3}\right)$.

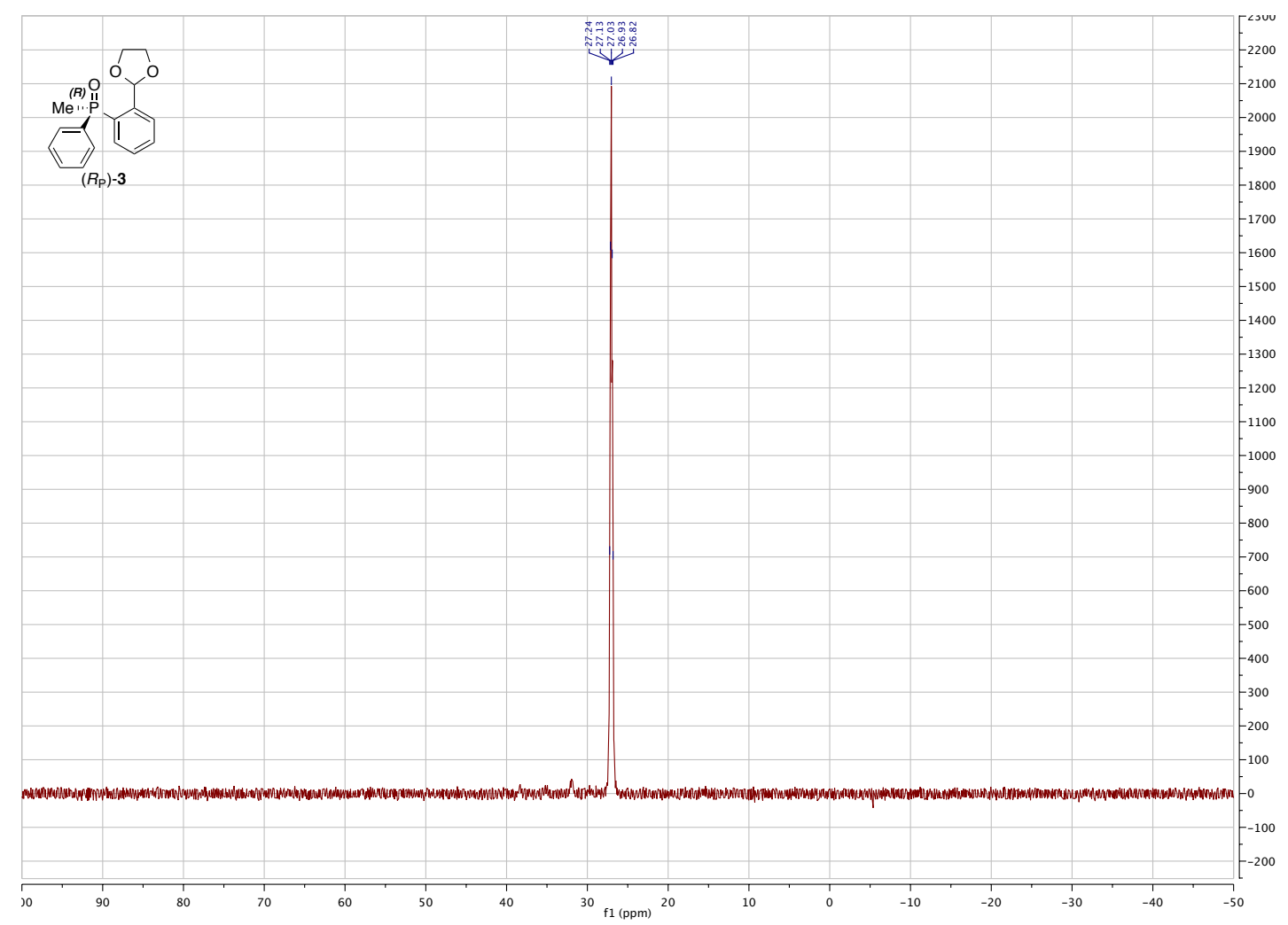

Figure S23: ${ }^{13} \mathrm{C}\left\{{ }^{1} \mathrm{H}\right\}$ NMR spectrum of $\left(R_{\mathrm{P}}\right)-3\left(75 \mathrm{MHz}, \mathrm{CDCl}_{3}\right)$.

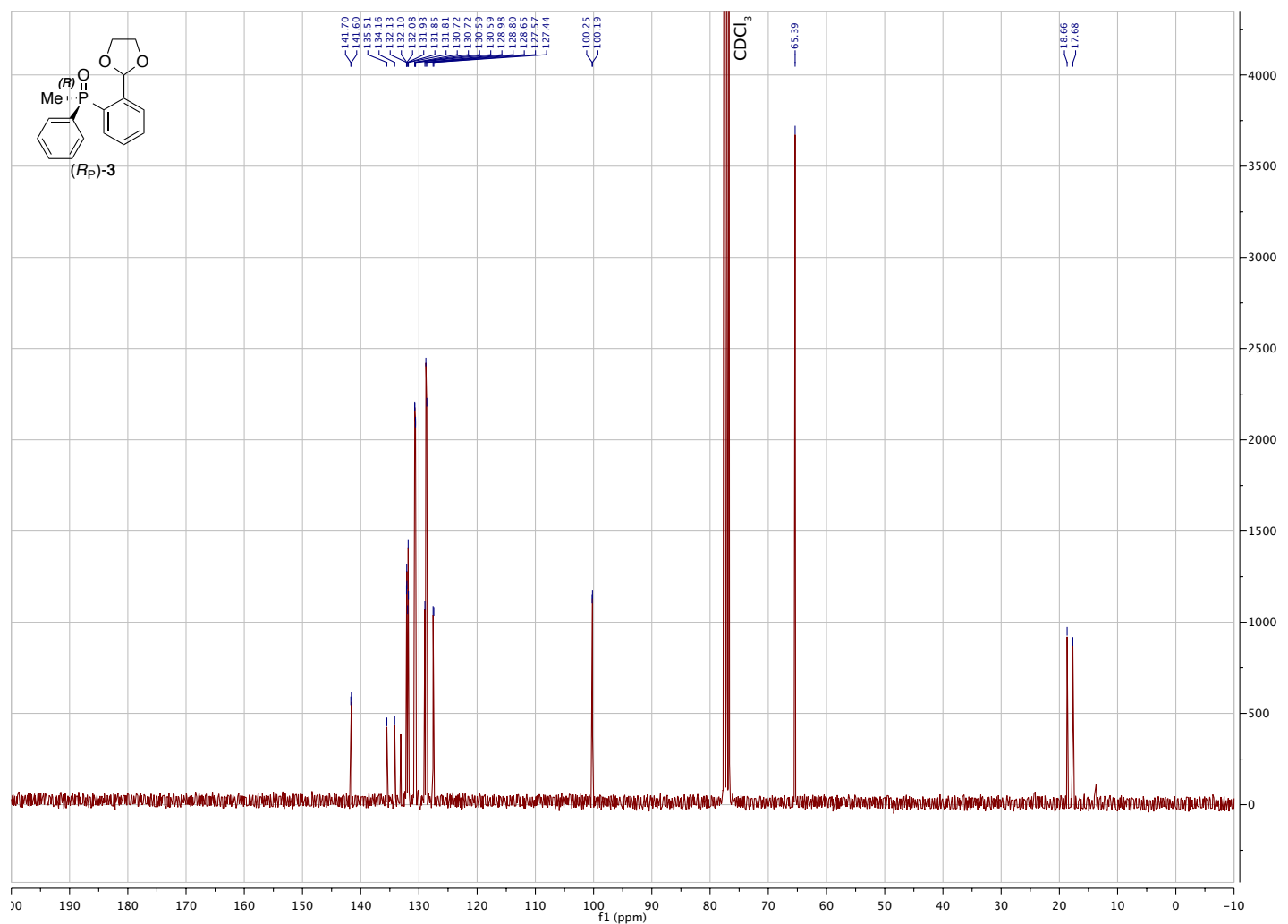


Figure S24: HPLC trace of $\left(R_{\mathrm{P}}\right)$-3.

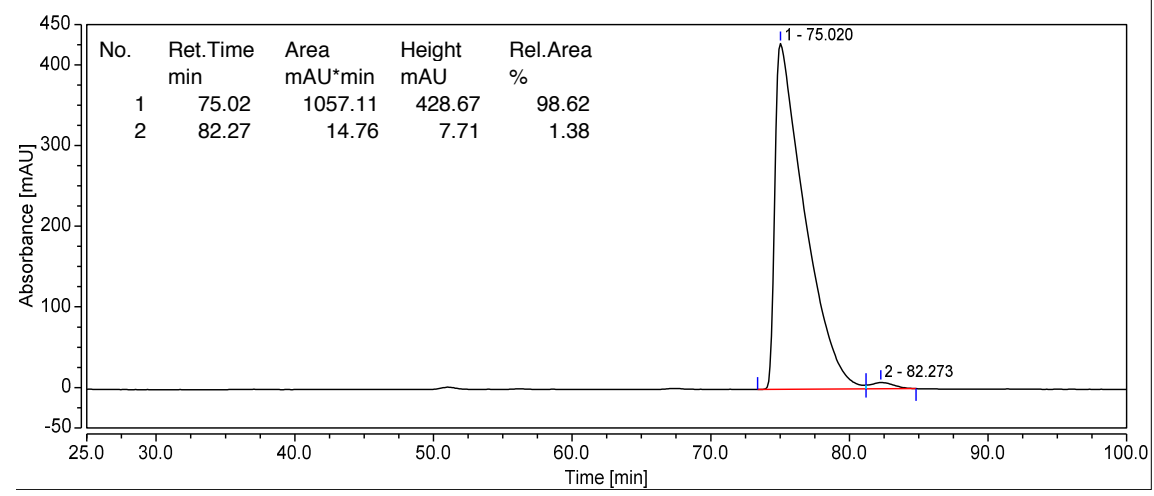

Figure S25: HPLC trace of racemic 3.
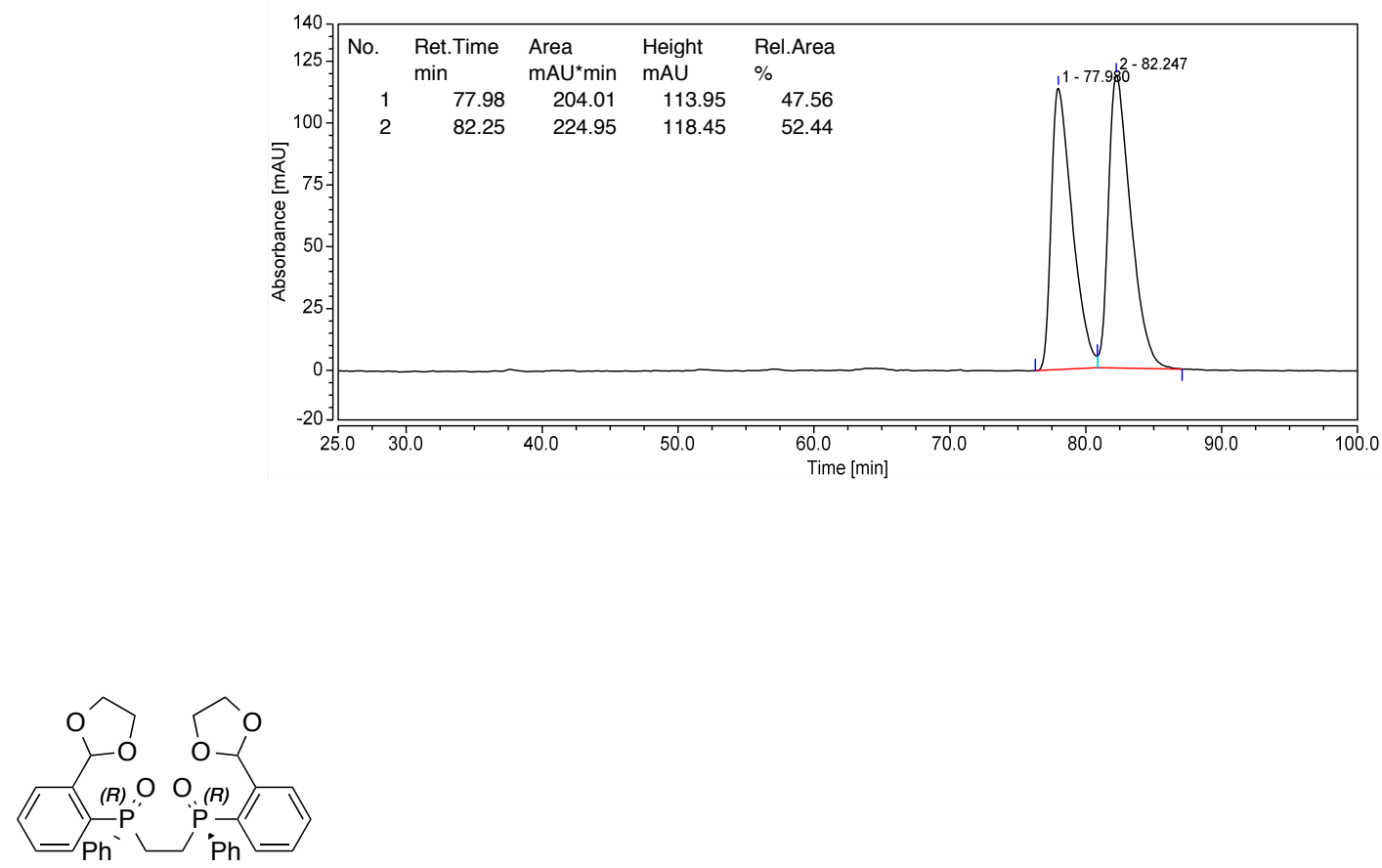

$\left(R_{\mathrm{P}}, R_{\mathrm{P}}\right)-4$

\section{Synthesis of $\left(1 R, 1^{\prime} R\right)$-Ethane-1,2-diylbis((2-(1,3-dioxolan-2-yl)phenyl)-}

(phenyl)phosphine oxide), $\left(\boldsymbol{R}_{\mathbf{P}}, \boldsymbol{R}_{\mathbf{P}}\right)$-4. A flame-dried $250 \mathrm{~mL}$ Schlenk was charged with diisopropylamine (12.4 mL, $88.5 \mathrm{mmol}, 1.5 \mathrm{equiv})$ and THF $(48 \mathrm{~mL})$, and then cooled to $0{ }^{\circ} \mathrm{C}$. After $5 \mathrm{~min}, n$-butyllithium $(51.6 \mathrm{~mL}, 1.6 \mathrm{M}$ in hexanes, $82.6 \mathrm{mmol}, 1.4$ equiv) was added, and the solution was stirred for $30 \mathrm{~min}$ at $0{ }^{\circ} \mathrm{C}$. In a separate flame-dried $500 \mathrm{~mL}$ two-neck round-bottom flask, $\left(R_{\mathrm{P}}\right)-3(17.0 \mathrm{~g}, 59.0 \mathrm{mmol})$ was dissolved in THF $(220 \mathrm{~mL})$ and cooled to $-78{ }^{\circ} \mathrm{C}($ Note: If precipitation occurs, $\left(R_{\mathrm{P}}\right)-\mathbf{3}$ has to be dissolved by warming to room temperature). After 5 min, the 
first solution was added dropwise over $15 \mathrm{~min}$ at $-78^{\circ} \mathrm{C}$ using a cannula (Note: Slow addition is crucial to prevent side reactions). After $5 \mathrm{~min}$, copper(II) chloride (13.5 g, $100 \mathrm{mmol}, 1.7$ equiv) was added in one portion, and the solution was warmed to room temperature overnight. After removal of the solvent at the rotary evaporator $\left(20 \mathrm{mbar}, 40{ }^{\circ} \mathrm{C}\right)$, the crude product was dissolved in chloroform $(1000 \mathrm{~mL})$ and washed three times with $10 \%$ aqueous $\mathrm{NH}_{3}$ solution $(3 \times 300 \mathrm{~mL}$; each time, the aqueous phase was extracted twice with chloroform $(2 \times 100 \mathrm{~mL}))$ and once with water (300 $\mathrm{mL}$; the aqueous phase was extracted twice with chloroform $(2 \times 100 \mathrm{~mL}))$. The combined organic phases were dried over $\mathrm{MgSO}_{4}$, filtered, and the solvent was removed at the rotary evaporator $\left(20\right.$ mbar, $\left.40{ }^{\circ} \mathrm{C}\right)$. Purification by flash column chromatography on silica gel $\left(8.5 \times 30 \mathrm{~cm}\right.$, gradient $\mathrm{CH}_{2} \mathrm{Cl}_{2}: \mathrm{MeOH}=100: 0$ to $\left.93: 7\right)$ afforded the product as an off-white solid. Yield: $13.5 \mathrm{~g}(80 \%$, d.r. $\approx 12: 1) .{ }^{1} \mathbf{H}$ NMR $\left(300 \mathrm{MHz}, \mathrm{CDCl}_{3}\right): \delta 7.78\left(d,{ }^{3} J_{\mathrm{H}, \mathrm{H}},=7.5 \mathrm{~Hz}, 2 \mathrm{H}\right.$, $\operatorname{Ar}-H), 7.72-7.62(m, 4 \mathrm{H}, \mathrm{Ar}-H), 7.61-7.35(m, 12 \mathrm{H}, \mathrm{Ar}-H), 6.45\left(s, 2 \mathrm{H}, \mathrm{CH}\left(\mathrm{OCH}_{2}\right)_{2}\right), 4.01-$ $3.79\left(m, 8 \mathrm{H}, \mathrm{CH}(\mathrm{OCHH})_{2}\right), 2.90-2.48(m, 4 \mathrm{H}, \mathrm{PCHH}) .{ }^{31} \mathbf{P}\left\{{ }^{1} \mathbf{H}\right\} \mathbf{N M R}\left(122 \mathrm{MHz}, \mathrm{CDCl}_{3}\right)$ : $\delta 35.6(s) .{ }^{13} \mathbf{C}\left\{{ }^{1} \mathbf{H}\right\}$ NMR $\left(75 \mathrm{MHz}, \mathrm{CDCl}_{3}\right): \delta 142.4\left(t,{ }^{2} J_{\mathrm{P}, \mathrm{C}}=3.5 \mathrm{~Hz}\right.$, arom.), 133.2 (m, arom.), 132.23 ( $m$, arom.), 132.17 ( $t, J_{\mathrm{P}, \mathrm{C}}=5.4 \mathrm{~Hz}$, arom.), 132.0 ( $m$, arom.), $131.0\left(t, J_{\mathrm{P}, \mathrm{C}}=4.8 \mathrm{~Hz}\right.$, arom.), $130.6\left(t,{ }^{1} J_{\mathrm{P}, \mathrm{C}}=47.3 \mathrm{~Hz}\right.$, arom. $), 129.0\left(t, J_{\mathrm{P}, \mathrm{C}}=5.8 \mathrm{~Hz}\right.$, arom. $), 128.7\left(t, J_{\mathrm{P}, \mathrm{C}}=5.9 \mathrm{~Hz}\right.$, arom. $)$, $127.7\left(t, J_{\mathrm{P}, \mathrm{C}}=4.8 \mathrm{~Hz}\right.$, arom. $), 100.2\left(t,{ }^{3} J_{\mathrm{P}, \mathrm{C}}=2.0 \mathrm{~Hz}, \mathrm{CH}\left(\mathrm{OCH}_{2}\right)_{2}\right), 65.33\left(\mathrm{CH}\left(\mathrm{OCH}_{2}\right)_{2}\right), 65.30$ $\left(\mathrm{CH}\left(\mathrm{OCH}_{2}\right)_{2}\right), 18.2\left(t,{ }^{1} J_{\mathrm{P}, \mathrm{C}}=33.8 \mathrm{~Hz}, \mathrm{PCH}_{2}\right)$. Melting Point: $146{ }^{\circ} \mathrm{C}$. IR (liquid film, $\left.\mathrm{cm}^{-1}\right)$ : 3058 (C-H), 3023 (C-H), 2955 (C-H), 2887 (C-H), $2227(\mathrm{P}=\mathrm{O}), 1725,1638,1596,1572,1492$, 1473, 1437, 1412, 1396, 1349, 1333, 1312, 1285, 1171, 1132, 1112, 1092, 1061, 1027. EA: Calcd. for $\mathrm{C}_{32} \mathrm{H}_{32} \mathrm{O}_{6} \mathrm{P}_{2}$ : C, 66.90; H, 5.61; found: C, 66.87; H, 5.59. HRMS (ESI): Calcd. for $\mathrm{C}_{32} \mathrm{H}_{33} \mathrm{O}_{6} \mathrm{P}_{2}$ $m / z=575.1747$, found $m / z=575.1744[\mathrm{M}+\mathrm{H}]^{+}$. HPLC: No separation obtained with standard columns. $[\alpha]_{\mathbf{D}}{ }^{20}:-5.6\left(\mathrm{c}=1.0, \mathrm{CH}_{2} \mathrm{Cl}_{2}\right)$. 
Figure S26: ${ }^{1} \mathrm{H}$ NMR spectrum of $\left(R_{\mathrm{P}}, R_{\mathrm{P}}\right)-4\left(300 \mathrm{MHz}, \mathrm{CDCl}_{3}\right)$.

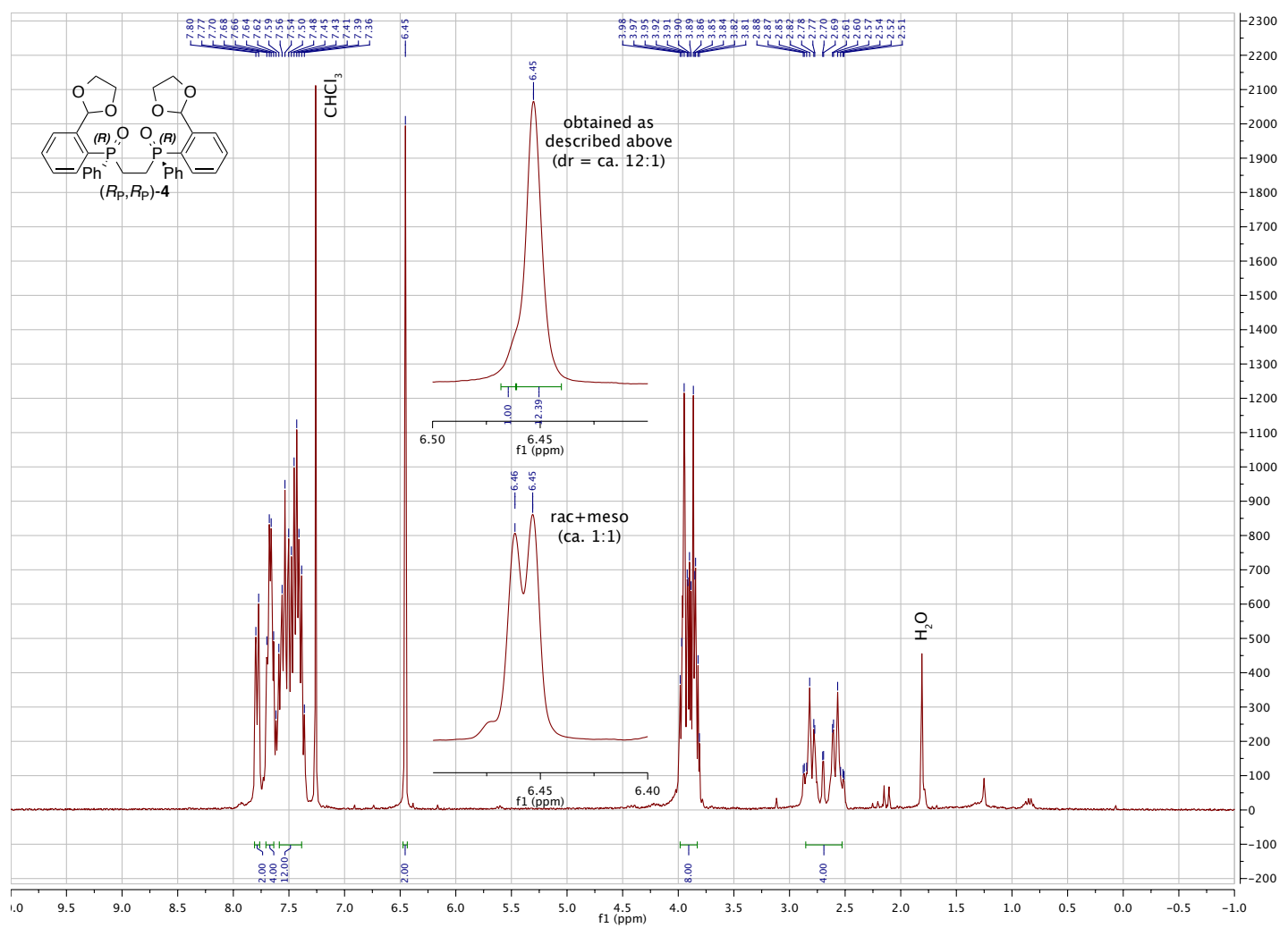

Figure S27: ${ }^{31} \mathrm{P}\left\{{ }^{1} \mathrm{H}\right\}$ NMR spectrum of $\left(R_{\mathrm{P}}, R_{\mathrm{P}}\right)-\mathbf{4}\left(122 \mathrm{MHz}, \mathrm{CDCl}_{3}\right)$.

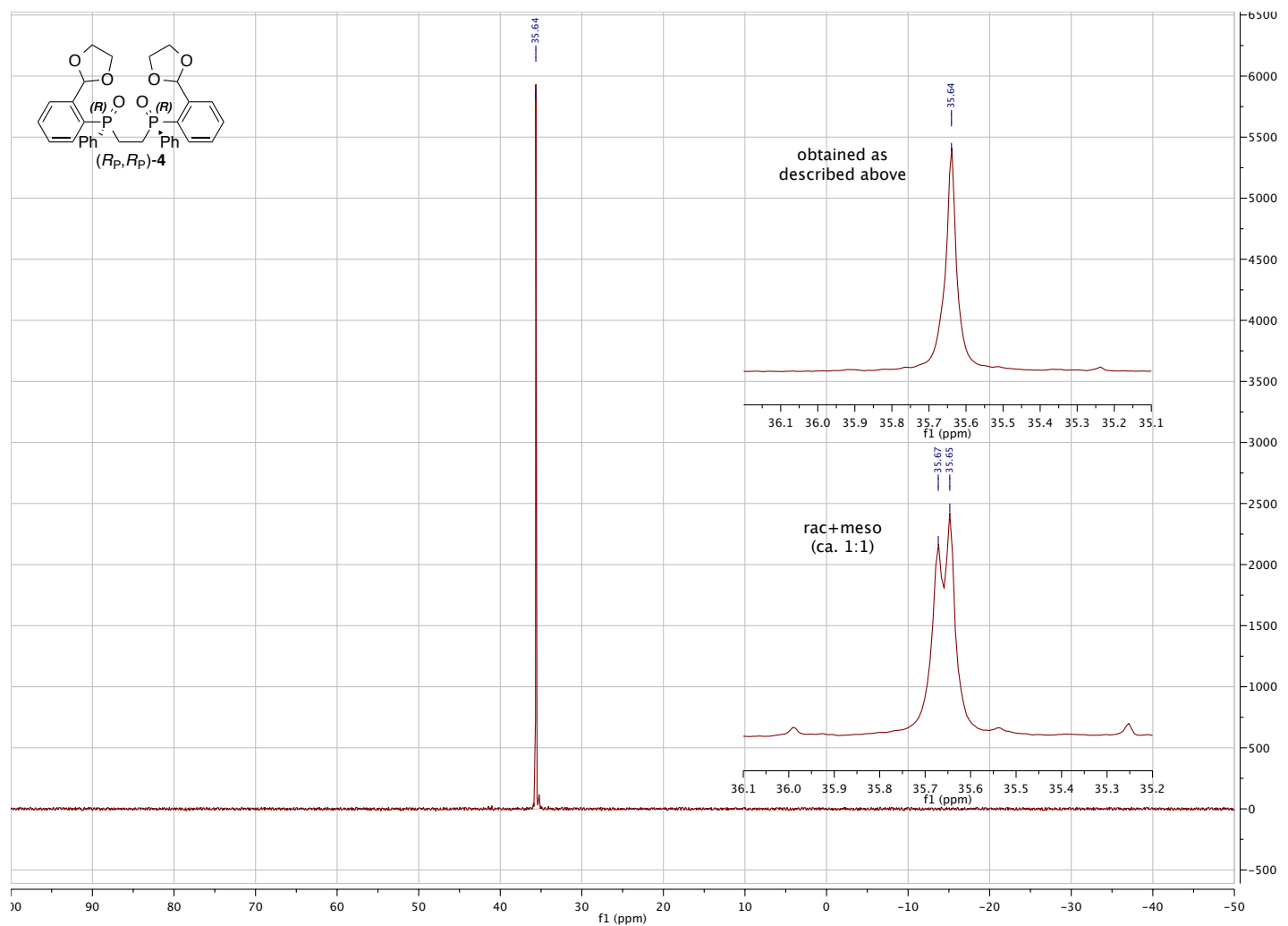


Figure S28: ${ }^{13} \mathrm{C}\left\{{ }^{1} \mathrm{H}\right\}$ NMR spectrum of $\left(R_{\mathrm{P}}, R_{\mathrm{P}}\right)-4\left(75 \mathrm{MHz}, \mathrm{CDCl}_{3}\right)$.

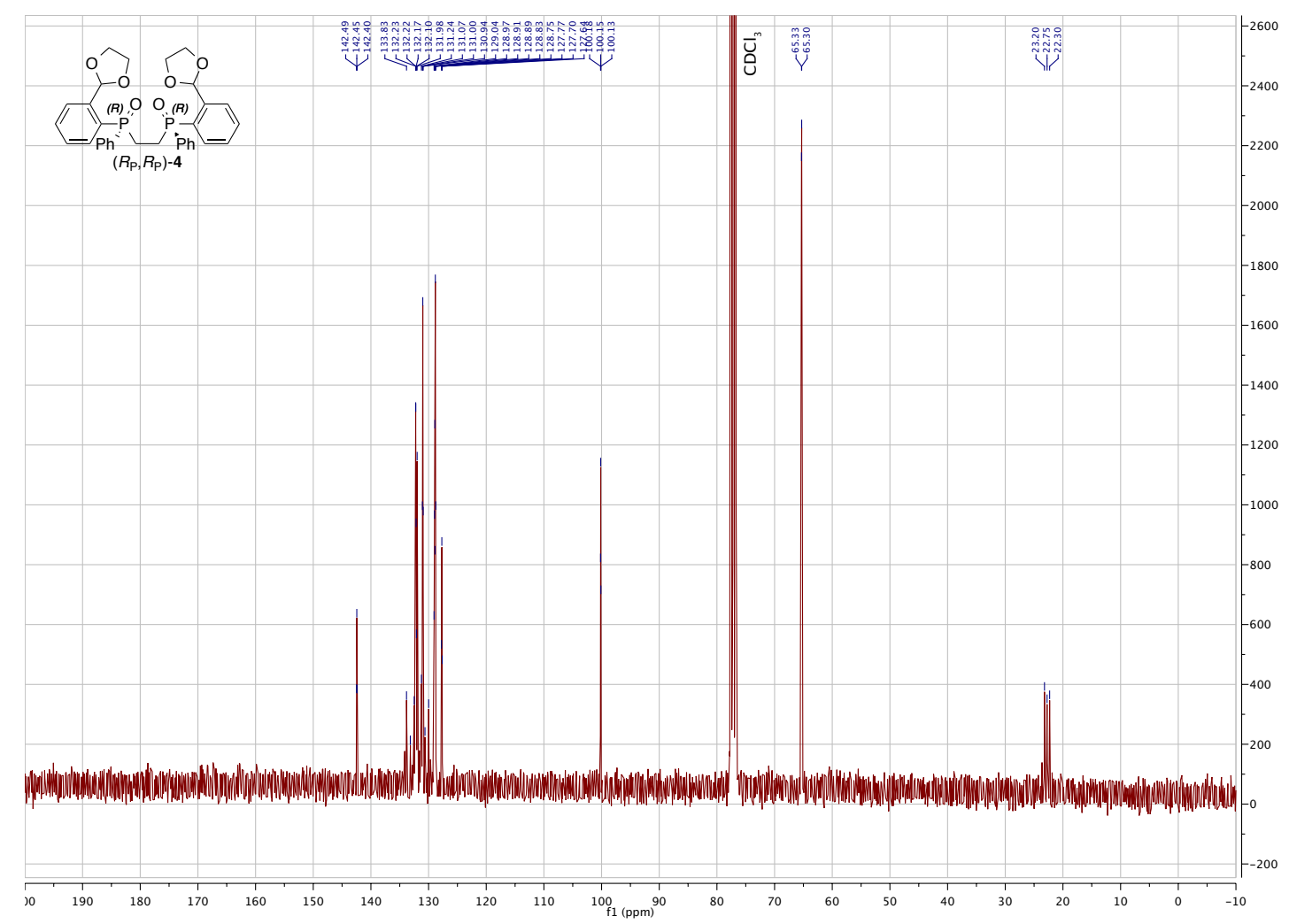

\section{X-Ray Structure of $\left(1 R, 1^{\prime} R\right)$-Ethane-1,2-diylbis((2-(1,3-dioxolan-2-yl)phenyl)(phen-}

yl)phosphine oxide), $\left(\boldsymbol{R}_{\mathbf{P}}, \boldsymbol{R}_{\mathrm{P}}\right)-\mathbf{4}$. Colorless crystals of $\left(R_{\mathrm{P}}, R_{\mathrm{P}}\right)-\mathbf{4}$ were obtained by crystallization from hot BuOAc. Crystal data for $\mathrm{C}_{32} \mathrm{H}_{32} \mathrm{O}_{6} \mathrm{P}_{2}$ : block $(0.31 \times 0.23 \times 0.21 \mathrm{~mm})$, triclinic, $P 1$, cell dimensions (100 K) $a=8.4358(4), b=8.6021(4), c=9.8323(4) \AA, \alpha=104.212(3), \beta=98.226(3), \gamma$ $=96.026(3)^{\circ}$ and $V=677.20(5) \AA^{3}$ with $Z=1, D_{\mathrm{c}}=1.409 \mathrm{Mg} / \mathrm{m}^{3}, \mu=0.207 \mathrm{~mm}^{-1}$ (Mo $\mathrm{K}_{\alpha}$, graphite monochromated), $\lambda=0.71073 \AA, F(000)=302$. The data were collected at $100 \mathrm{~K}$ on a Bruker AXS SMART APEX2 platform in the $\theta$ range $2.17-29.57^{\circ}$. The structure was solved with SHELXS using direct methods. Of the $31^{\prime} 764$ measured reflections with index ranges $-11 \leq h \leq 11$, $-11 \leq k \leq 11,-13 \leq l \leq 13,7^{\prime} 614$ independent reflections $\left(R_{\text {int }}=0.062\right)$ were used in the refinement (full-matrix least squares on $F^{2}$ ) with anisotropic displacement parameters for all non-H atoms. 
Hydrogen atoms were introduced at calculated positions and refined with the riding model and individual isotropic thermal parameters for each group. The asymmetric unit contains one molecule of $\left(R_{\mathrm{P}}, R_{\mathrm{P}}\right)-4$. Final residuals were $R_{1}=0.0509$ (for 6'277 reflections with $\left.I>2 \sigma(I)\right)$ and $\mathrm{w} R_{2}=$ 0.1154 (all data), GOF $=0.973$, absolute structure parameter $-0.02(7)$. Max. and min. difference peaks were +0.67 and $-0.29 \mathrm{e}^{-3}$, the largest and mean $\Delta / \sigma=0.000$ and 0.000 .

Figure S29: X-ray structure of $\left(R_{\mathrm{P}}, R_{\mathrm{P}}\right)-4$.
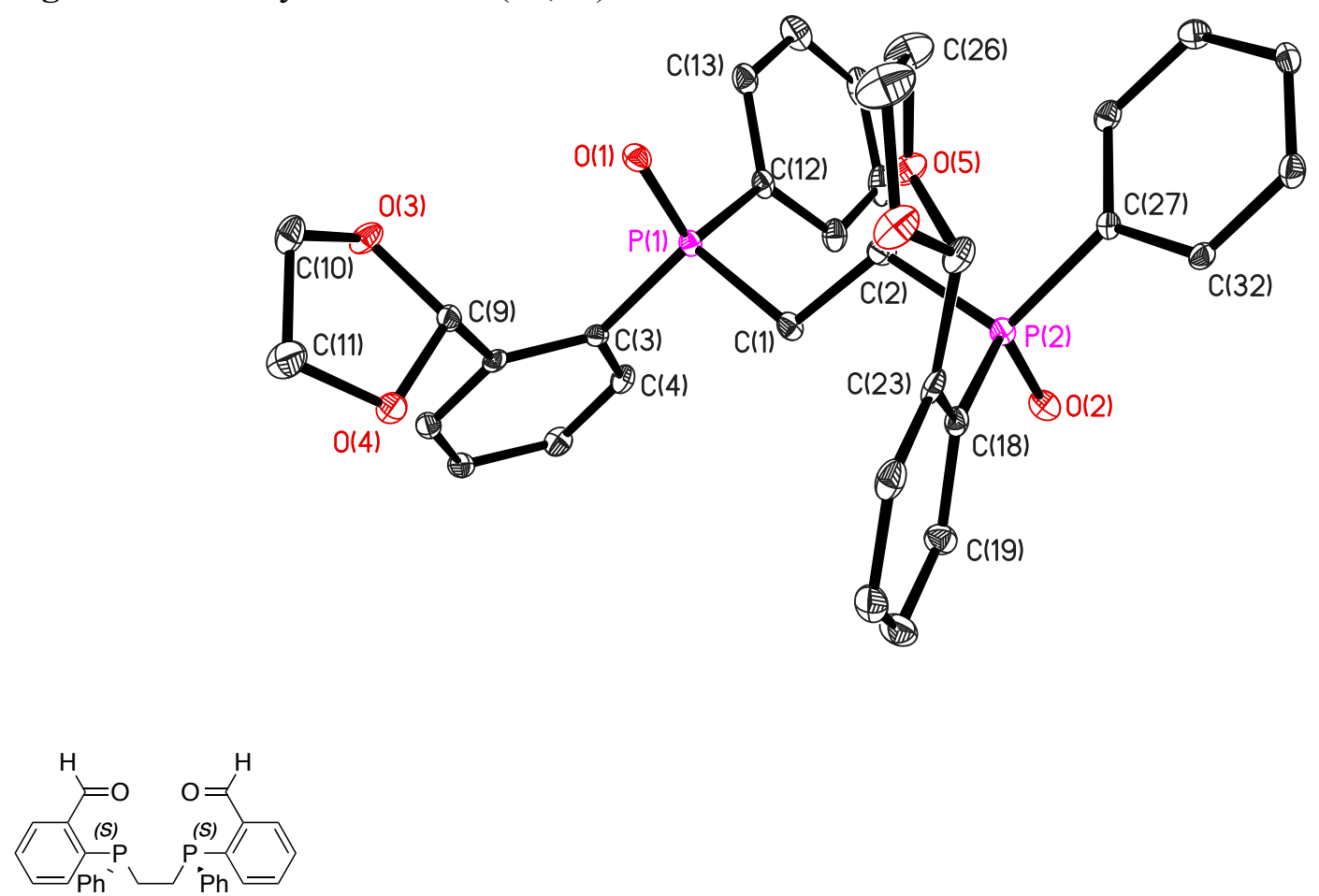

$\left(S_{\mathrm{P}}, S_{\mathrm{P}}\right)-5$

Synthesis of 2,2'-((1S,1'S)-Ethane-1,2-diylbis(phenylphosphanediyl))di-

benzaldehyde, $\left(\boldsymbol{S}_{\mathbf{P}}, \boldsymbol{S}_{\mathbf{P}}\right)$-5. A flame-dried $1000 \mathrm{~mL}$ two-neck round-bottom flask was charged with powdered $\left(R_{\mathrm{P}}, R_{\mathrm{P}}\right)-4(13.5 \mathrm{~g}, 23.5 \mathrm{mmol})$ and THF $(470 \mathrm{~mL})$, and then heated to $60{ }^{\circ} \mathrm{C}$. After $15 \mathrm{~min}$, titanium(IV) iso-propoxide $(42.8 \mathrm{~mL}, 141 \mathrm{mmol}, 6.0$ equiv) was added dropwise over 15 min. After $15 \mathrm{~min}$, poly(methylhydrosiloxane) (70.2 mL, average $\mathrm{M}_{\mathrm{n}}=1700-3200,50$ equiv) was added dropwise over $45 \mathrm{~min}$, and then the solution was stirred for $12 \mathrm{~h}$ at $60{ }^{\circ} \mathrm{C}$ to give a deep blue solution (Notes: The addition of poly(methylhydrosiloxane) leads to an exothermic reaction; fast addition of poly(methylhydrosiloxane) can lead to precipitation of starting material). After 
cooling to room temperature, the volatiles were removed using an external cooling trap. Hexane $\left(350 \mathrm{~mL}\right.$ ) was added, and the suspension was stirred for $30 \mathrm{~min}$ at $50{ }^{\circ} \mathrm{C}$ and then cooled to $-78{ }^{\circ} \mathrm{C}$ for $30 \mathrm{~min}$, which leads to crystallization of the reduced intermediate. The product was rapidly filtered off on top of a Celite ${ }^{\circledR}$ pad in a Young filter using a cannula (Notes: The filter was externally cooled by dry ice; Celite ${ }^{\circledR}$ was used to prevent clogging of the filter) and washed twice with cold hexane $(2 \times 15 \mathrm{~mL})$. The solid residue was dissolved in THF $(430 \mathrm{~mL})$ and filtered through the Celite ${ }^{\circledR}$ pad into a $2000 \mathrm{~mL}$ two-neck round-bottom flask. Aqueous $5 \% \mathrm{HCl}(215 \mathrm{~mL}$; water was degassed by bubbling nitrogen through for $1 \mathrm{~h}$ ) was added, and the solution was vigorously stirred for $5 \mathrm{~h}$ at room temperature. Saturated aqueous $\mathrm{NaHCO}_{3}(400 \mathrm{~mL}$; degassed by bubbling nitrogen through for $1 \mathrm{~h}$ ) was added thereto (Note: Gas evolution), and the organic solvent was removed at the rotary evaporator $\left(120 \mathrm{mbar}, 40^{\circ} \mathrm{C}\right)$. The aqueous phase was extracted three times with dichloromethane $(3 \times 400 \mathrm{~mL}$; degassed by bubbling nitrogen through for $1 \mathrm{~h})$. The combined organic phases were dried over $\mathrm{MgSO}_{4}$, filtered and the solvent was removed at the rotary evaporator $\left(20 \mathrm{mbar}, 40^{\circ} \mathrm{C}\right)$. The orange product was purified by rapid filtration through a silica gel plug $($ EtOAc $:$ hexane $=1: 3$; solvent degassed by bubbling nitrogen through for $1 \mathrm{~h}$ ) (Note: The product appears as a yellow band, whereas a red band (baseline) indicates oxidized product) to afford the crude product as a yellow oil. Crude yield: $8.43 \mathrm{~g}(79 \%$, d.r. $=6.4: 1)$. ${ }^{31} \mathbf{P}\left\{{ }^{1} \mathbf{H}\right\}$ NMR $\left(122 \mathrm{MHz}, \mathrm{CD}_{2} \mathrm{Cl}_{2}\right): \delta-22.0$ (like-5, 86\%), -22.2 (meso-5, 14\%). The crude product was transferred to a $1000 \mathrm{~mL}$ round-bottom flask and suspended in $\mathrm{MeOH}(400 \mathrm{~mL}$; degassed by bubbling nitrogen through for $1 \mathrm{~h}$ ). The suspension was heated to reflux for 2 min using a heat gun (Note: Prolonged heating (i.e. using an oil bath) should be avoided as it leads to epimerization), allowed to cool to room temperature overnight, and then placed in a freezer for $6 \mathrm{~h}$. Filtration and washing with ice-cold $\mathrm{MeOH}(2 \times 20 \mathrm{~mL})$ afforded the pure title product $(>98 \%$ pure by $\left.{ }^{31} \mathrm{P}\left\{{ }^{1} \mathrm{H}\right\} \mathrm{NMR}\right)$ as a yellow solid. Yield: $5.28 \mathrm{~g}(49 \%, 99 \%$ ee, d.r. $>99: 1) .{ }^{1} \mathbf{H}$ NMR (300 MHz, $\left.\mathrm{CD}_{2} \mathrm{Cl}_{2}\right): \delta 10.55\left(t,{ }^{4} J_{\mathrm{P}, \mathrm{H}}=2.7 \mathrm{~Hz}, 2 \mathrm{H}, \mathrm{O}=\mathrm{C} H\right), 7.94-7.85(m, 2 \mathrm{H}, \mathrm{Ar}-H), 7.55-7.45(m, 4 \mathrm{H}$, 
Ar- $H), 7.37-7.25(m, 12 \mathrm{H}, \operatorname{Ar}-H), 2.29-2.01(m, 4 \mathrm{H}, \mathrm{PCHH}) .{ }^{31} \mathbf{P}\left\{{ }^{1} \mathbf{H}\right\}$ NMR $(122 \mathrm{MHz}$, $\left.\mathrm{CD}_{2} \mathrm{Cl}_{2}\right): \delta-22.0\left(\right.$ s, like-5), -22.2 (s, meso-5), d.r. > 99:1. ${ }^{13} \mathbf{C}\left\{{ }^{1} \mathbf{H}\right\}$ NMR $\left(75 \mathrm{MHz}, \mathrm{CD}_{2} \mathrm{Cl}_{2}\right)$ : $\delta 192.4$ ( $m$, CHO), 142.6 ( $m$, arom.), 139.5 ( $m$, arom.), 137.9 (m, arom.), 134.1 (arom.), 133.7 ( $m$, arom.), 132.5 (arom.), 131.3 (arom.), 129.6 (arom.), 129.4 (arom.), 129.2 (m, arom.), 24.2 (m, $\mathrm{PCH}_{2}$ ). Melting Point: $128{ }^{\circ} \mathrm{C}$. IR (liquid film, $\mathrm{cm}^{-1}$ ): $3054(\mathrm{C}-\mathrm{H}), 2823(\mathrm{C}-\mathrm{H}), 2739(\mathrm{C}-\mathrm{H})$, $1693(\mathrm{C}=\mathrm{O}), 1584,1561,1482,1461,1433,1422,1386,1294,1258,1197,1163,1117,1094$. EA: Calcd. for $\mathrm{C}_{28} \mathrm{H}_{24} \mathrm{O}_{2} \mathrm{P}_{2}$ : C, 74.00; H, 5.32; found: C, 73.73; H, 5.30. HRMS (ESI): Calcd. for $\mathrm{C}_{28} \mathrm{H}_{24} \mathrm{NaO}_{2} \mathrm{P}_{2} m / z=477.1144$, found $m / z=477.1135[\mathrm{M}+\mathrm{Na}]^{+}$. HPLC: No separation obtained with standard columns. $[\boldsymbol{\alpha}]_{\mathbf{D}}{ }^{20}:+35.6\left(\mathrm{c}=1.0, \mathrm{CH}_{2} \mathrm{Cl}_{2}\right)$. Analytical data are in agreement with literature data. ${ }^{\mathrm{S} 3}$

Figure S30: ${ }^{1} \mathrm{H}$ NMR spectrum of $\left(S_{\mathrm{P}}, S_{\mathrm{P}}\right)-5\left(300 \mathrm{MHz}, \mathrm{CD}_{2} \mathrm{Cl}_{2}\right)$.

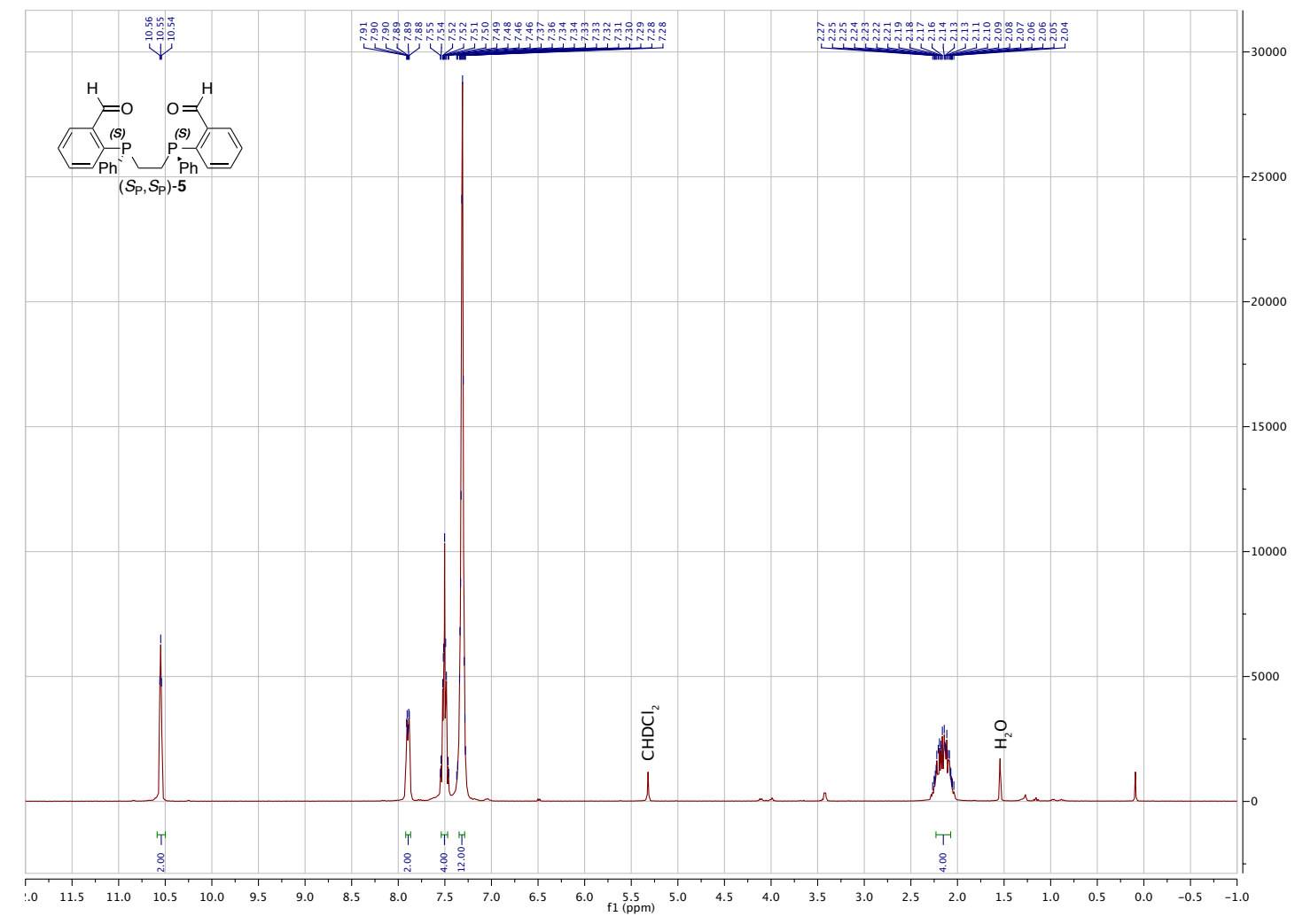


Figure S31: ${ }^{31} \mathrm{P}\left\{{ }^{1} \mathrm{H}\right\}$ NMR spectrum of $\left(S_{\mathrm{P}}, S_{\mathrm{P}}\right)-5\left(122 \mathrm{MHz}, \mathrm{CD}_{2} \mathrm{Cl}_{2}\right)$.

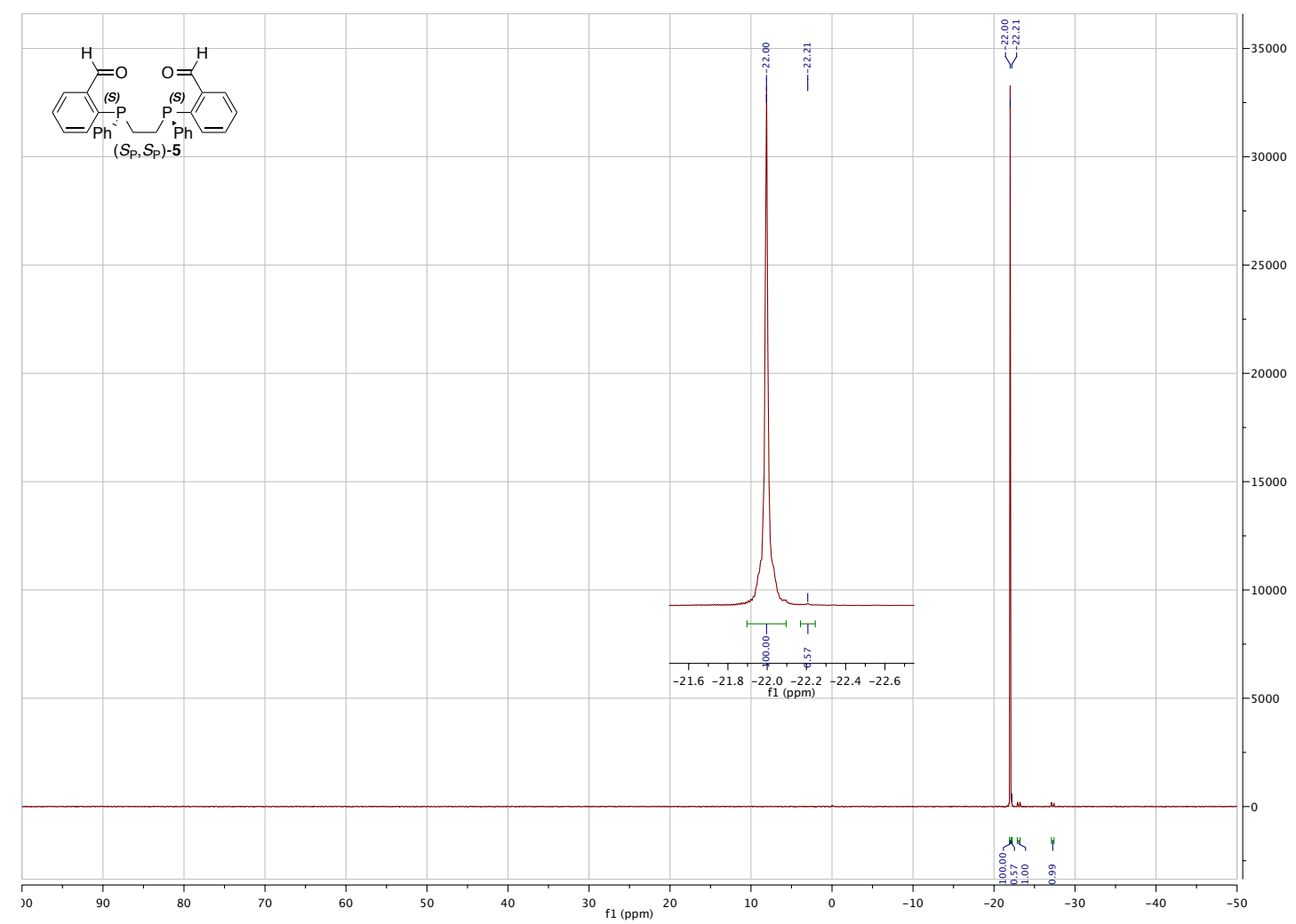

Figure S32: ${ }^{13} \mathrm{C}\left\{{ }^{1} \mathrm{H}\right\}$ NMR spectrum of $\left(S_{\mathrm{P}}, S_{\mathrm{P}}\right)-5\left(75 \mathrm{MHz}, \mathrm{CD}_{2} \mathrm{Cl}_{2}\right)$.

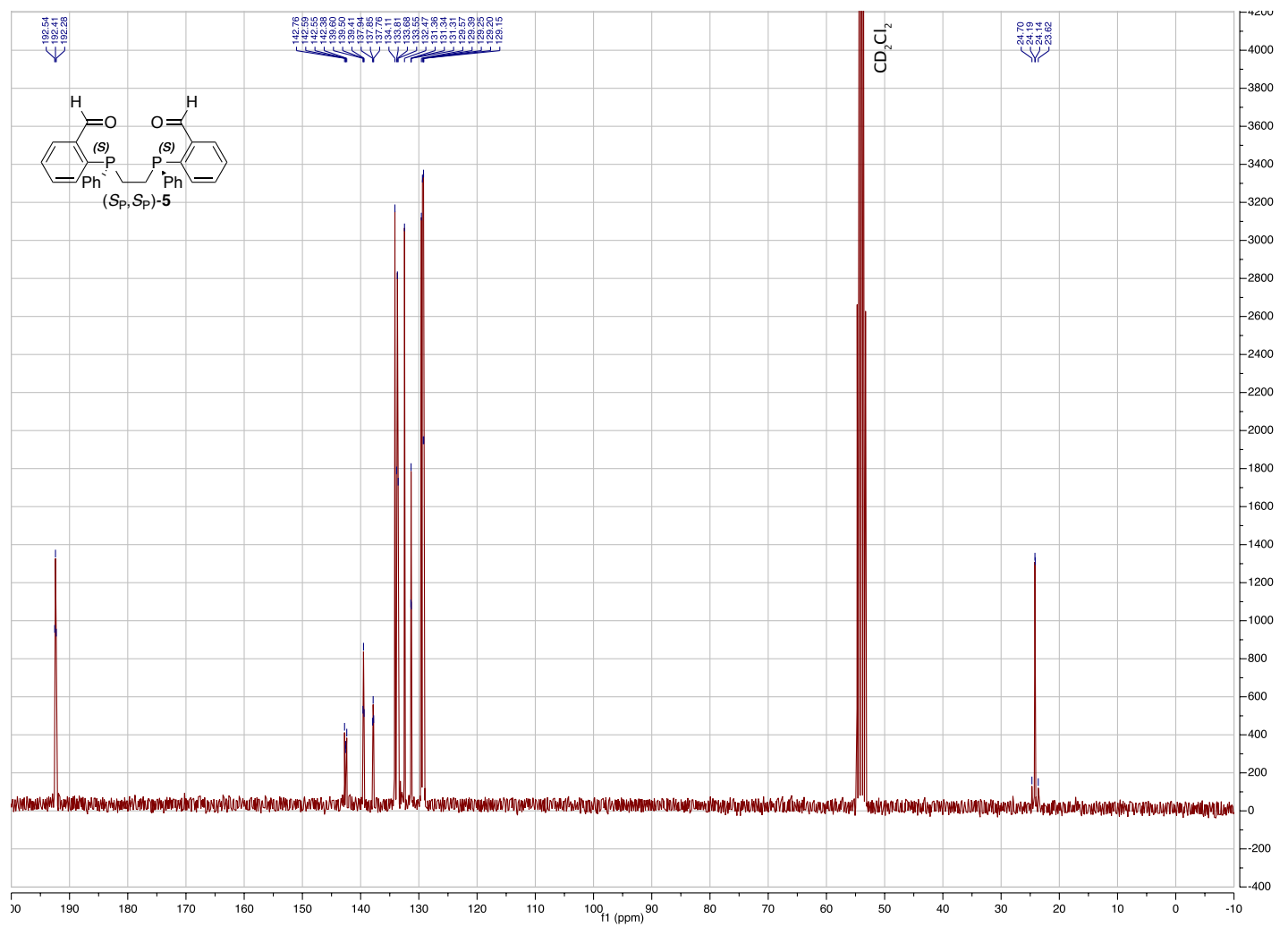




\section{Determination of the Enantiomeric Purity of 2,2'-((1S,1'S)-Ethane-1,2-diylbis-}

(phenylphosphanediyl))dibenzaldehyde, $\left(S_{\mathrm{P}}, S_{\mathrm{P}}\right)-5$. For the determination of the enantiomeric excess, the product was converted to $(((1 S, 1$ 'S)-ethane-1,2-diylbis(phenylphosphinediyl))bis(2,1phenylene))dimethanol diborane following a literature procedure. ${ }^{\mathrm{S} 3}$ A flame-dried $20 \mathrm{~mL}$ Schlenk flask was charged with $\left(S_{\mathrm{P}}, S_{\mathrm{P}}\right)-5(25.0 \mathrm{mg}, 55.0 \mu \mathrm{mol})$ and THF $(2.2 \mathrm{~mL})$, and then cooled to $0{ }^{\circ} \mathrm{C}$. After $5 \mathrm{~min}$, sodium borohydride (10.4 mg, $275 \mu \mathrm{mol}, 5.0$ equiv) was added, and the solution was stirred at $0{ }^{\circ} \mathrm{C}$ for $1 \mathrm{~h}$ to give a colorless solution. Borane dimethyl sulfide complex $(21 \mu \mathrm{L}$, $0.23 \mathrm{mmol}, 5.0$ equiv) was added, and the solution was stirred overnight at room temperature. The solution was diluted with THF (2 mL), and saturated aqueous $\mathrm{NH}_{4} \mathrm{Cl}(20 \mathrm{~mL})$ was added (Note: Gas evolution). The aqueous phase was separated and extracted three times with EtOAc $(3 \times 20 \mathrm{~mL})$. The combined organic phases were washed with saturated aqueous $\mathrm{NaCl}$ solution $(20 \mathrm{~mL})$, dried over $\mathrm{MgSO}_{4}$, filtered, and the solvent was removed at the rotary evaporator $\left(20 \mathrm{mbar}, 40{ }^{\circ} \mathrm{C}\right)$. Purification by flash column chromatography on silica gel $(1.5 \times 15 \mathrm{~cm}$, EtOAc : hex $=1: 1)$ afforded the title product as a white solid. Yield: $18.3 \mathrm{mg}(67 \%,>99 \%$ ee, d.r. = 33:1). HPLC: Chiralpak IC-3 (hexane : 2-PrOH $=80: 20$, flow rate $1.0 \mathrm{~mL} / \mathrm{min}, \lambda=230 \mathrm{~nm}$ ), retention times $t_{\mathrm{R}}($ minor $)=7.9 \mathrm{~min}, t_{\mathrm{R}}($ meso $)=8.9 \mathrm{~min}, t_{\mathrm{R}}($ major $)=11.0 \mathrm{~min}$. The presence of the meso isomer is attributed to racemization during the reduction. Analytical data are in agreement with literature data. $^{\text {S3 }}$

Figure S33: HPLC trace for the determination of enantiopurity of $\left(S_{\mathrm{P}}, S_{\mathrm{P}}\right)-\mathbf{5}$.

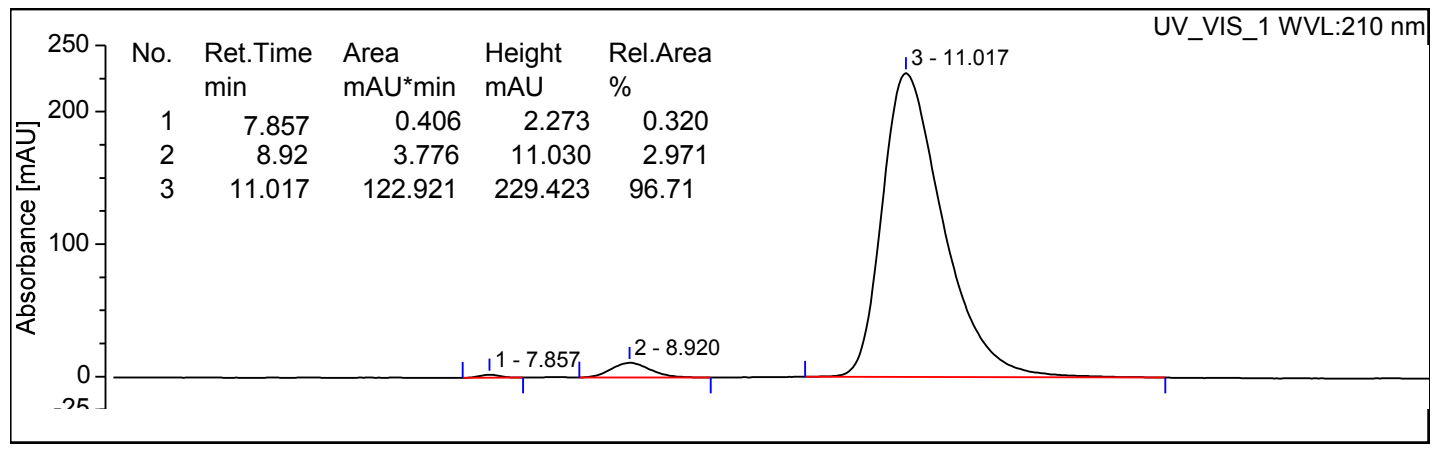




\section{Synthesis of Macrocycles and Iron(II) Complexes}

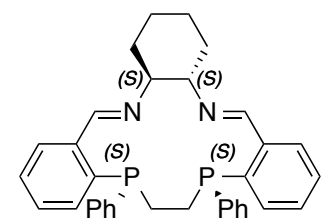

$\left(S_{\mathrm{P}}, S_{\mathrm{P}}, S_{\mathrm{C}}, S_{\mathrm{C}}\right)-6$

Synthesis of $(5 S, 8 S, 13 E, 14 a S, 18 \mathrm{a} S, 19 E)-5,8-D i p h e n y l-5,6,7,8,14 a, 15,16$, $17,18,18 a-d e c a h y d r o t r i b e n z o[b, f, l][1,4]$ diaza $[8,11]$ diphosphacyclotetradecine, $\left(S_{\mathrm{P}}, S_{\mathrm{P}}, S_{\mathrm{C}}, S_{\mathrm{C}}\right)-6$.

The title compound was prepared following a literature procedure. ${ }^{\mathrm{S} 3}$ A flame-dried $2000 \mathrm{~mL}$ twoneck round-bottom flask was charged with $\left(S_{\mathrm{P}}, S_{\mathrm{P}}\right)-5(4.35 \mathrm{~g}, 9.57 \mathrm{mmol})$, EtOH $(900 \mathrm{~mL}),(1 S, 2 S)-$ cyclohexane-1,2-diamine (1.09 g, $9.57 \mathrm{mmol}, 1.0$ equiv), and the solids were washed down with EtOH $(50 \mathrm{~mL})$. The solution was warmed to reflux using a heat gun until complete dissolution of the starting materials, and then stirred for $48 \mathrm{~h}$ at room temperature. The solution was concentrated to ca. $350 \mathrm{~mL}$ using an external cooling trap, during which the solution was kept at room temperature in a warm water bath. The solution was transferred to a flame-dried $500 \mathrm{~mL}$ two-neck round bottom flask, and further concentrated using an external cooling trap. When ca. $20 \mathrm{~mL}$ were left, the solution was placed in a freezer at $-18{ }^{\circ} \mathrm{C}$ overnight. The precipitated solid was filtered off in air and washed three times with ice-cold $\mathrm{EtOH}(3 \times 10 \mathrm{~mL})$ to give the title product as a white powder. Yield: $2.96 \mathrm{~g}(58 \%) .{ }^{1} \mathbf{H}$ NMR $\left(300 \mathrm{MHz}, \mathrm{CD}_{2} \mathrm{Cl}_{2}\right): \delta 8.95(\mathrm{~s}, 2 \mathrm{H}, \mathrm{N}=\mathrm{CH}), 7.79-7.73(\mathrm{~m}$, 2H, Ar-H), $7.43-7.17(m, 16 \mathrm{H}, \mathrm{Ar}-H), 3.51-3.40(m, 2 \mathrm{H}, \mathrm{N}-\mathrm{CH}), 2.27-2.10(m, 2 \mathrm{H}, \mathrm{CH})$, $1.99-1.78(m, 8 \mathrm{H}, \mathrm{CHH}), 1.60-1.45(m, 2 \mathrm{H}, \mathrm{CHH}) .{ }^{31} \mathbf{P}\left\{{ }^{1} \mathbf{H}\right\} \mathbf{N M R}\left(122 \mathrm{MHz}, \mathrm{CD}_{2} \mathrm{Cl}_{2}\right)$ : $\delta-27.2(s) .{ }^{13} \mathbf{C}\left\{{ }^{1} \mathbf{H}\right\}$ NMR $\left(75 \mathrm{MHz}, \mathrm{CD}_{2} \mathrm{Cl}_{2}\right): \delta 161.3\left(t,{ }^{3} J_{\mathrm{P}, \mathrm{C}}=11.5 \mathrm{~Hz}, \mathrm{~N}=C \mathrm{H}\right), 144.3(m$, arom.), 139.4 (m, arom.), 137.0 ( $m$, arom.), 133.1 (arom.), 132.1 (m, arom.), 130.1 (arom.), 129.8 (arom.), 128.9 (m, arom.), 128.4 (arom.), 128.3 (arom.), $73.4(\mathrm{~N}-\mathrm{CH}), 33.6\left(\mathrm{CH}_{2}\right), 25.0\left(\mathrm{CH}_{2}\right), 22.0$ ( $m, \mathrm{PCH}_{2}$ ). Melting Point: $178{ }^{\circ} \mathrm{C}$. IR (liquid film, $\mathrm{cm}^{-1}$ ): 3069 (C-H), 3050 (C-H), 3012 (C-H), 
$2927(\mathrm{C}-\mathrm{H}), 2854(\mathrm{C}-\mathrm{H}), 1635(\mathrm{C}=\mathrm{N}), 1585,1559,1481,1463,1446,1432,1370,1339,1330$, 1305, 1264, 1238, 1204, 1158, 1125, 1094, 1080, 1026. EA: Calcd. for $\mathrm{C}_{34} \mathrm{H}_{34} \mathrm{~N}_{2} \mathrm{P}_{2}$ : C, 76.67 ; H, 6.43; N, 5.26; found: C, 76.45; H, 6.33; N, 5.11. HRMS (ESI): Calcd. for $\mathrm{C}_{34} \mathrm{H}_{35} \mathrm{~N}_{2} \mathrm{P}_{2} m / z=$ 533.2270 , found $m / z=533.2262[\mathrm{M}+\mathrm{H}]^{+}$. Analytical data are in agreement with literature data. ${ }^{\mathrm{S} 3}$

Figure S34: ${ }^{1} \mathrm{H}$ NMR spectrum of $\left(S_{\mathrm{P}}, S_{\mathrm{P}}, S_{\mathrm{C}}, S_{\mathrm{C}}\right)-6\left(300 \mathrm{MHz}, \mathrm{CD}_{2} \mathrm{Cl}_{2}\right)$.

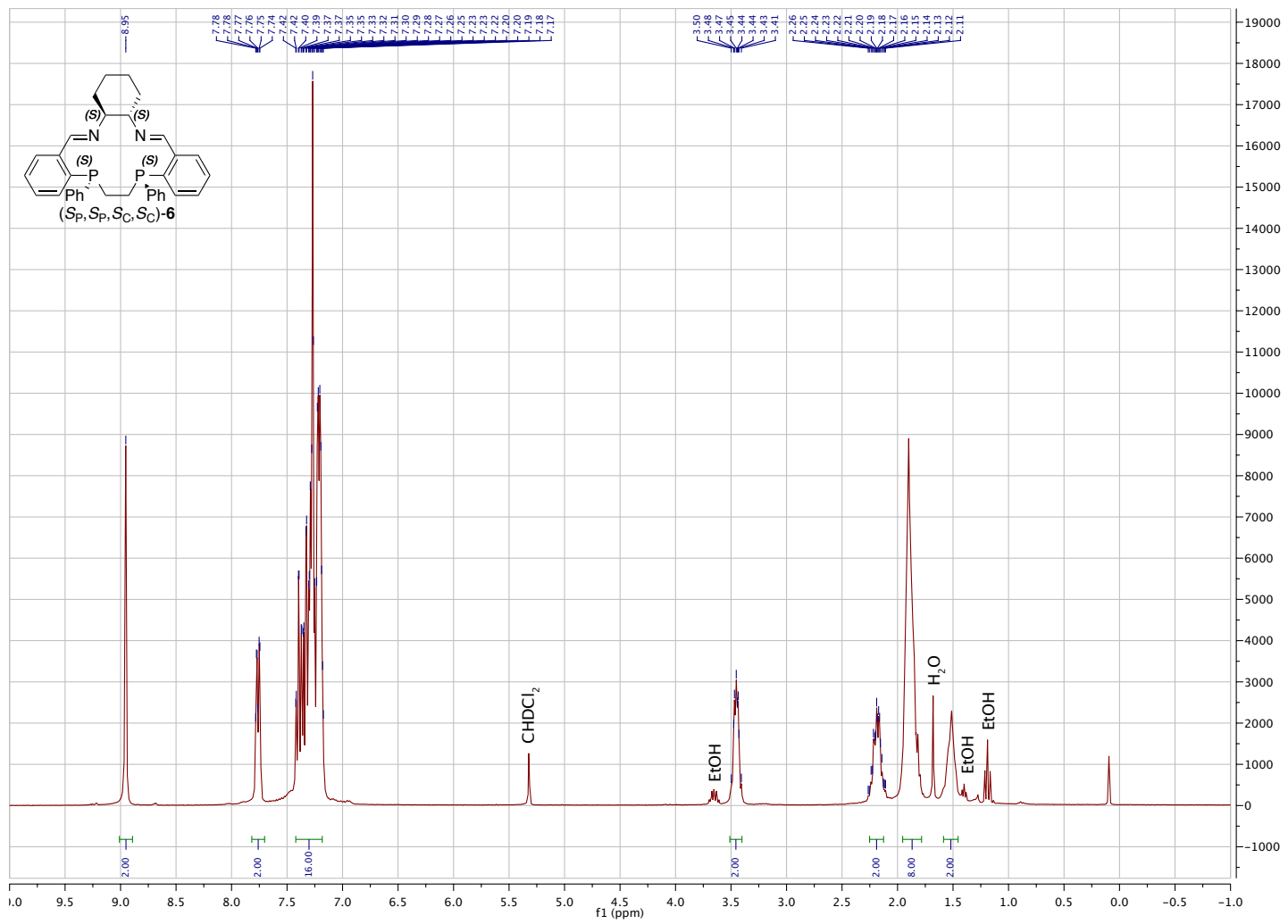


Figure S35: ${ }^{31} \mathrm{P}\left\{{ }^{1} \mathrm{H}\right\}$ NMR spectrum of $\left(S_{\mathrm{P}}, S_{\mathrm{P}}, S_{\mathrm{C}}, S_{\mathrm{C}}\right)-6\left(122 \mathrm{MHz}, \mathrm{CD}_{2} \mathrm{Cl}_{2}\right)$.

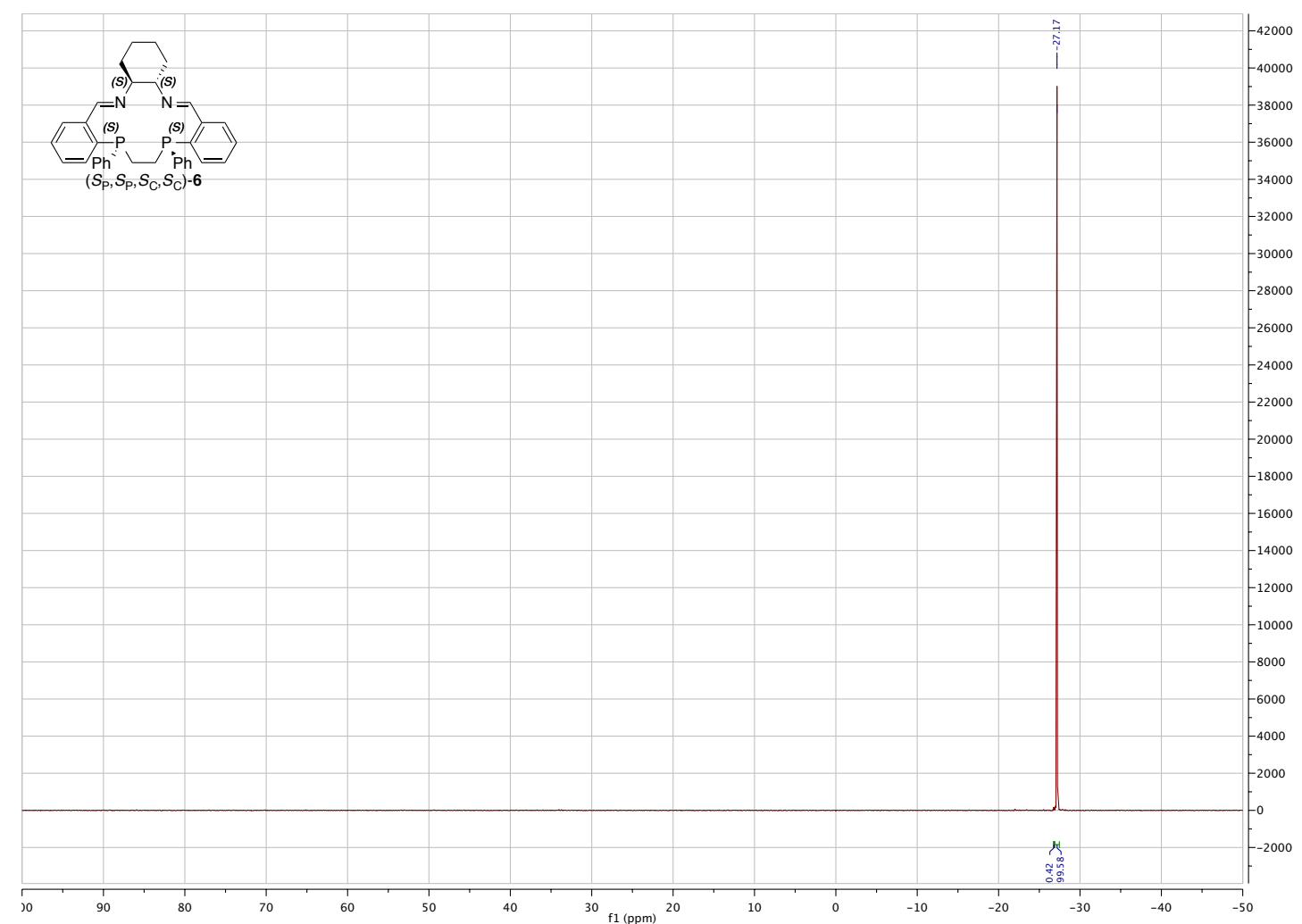

Figure S36: ${ }^{13} \mathrm{C}\left\{{ }^{1} \mathrm{H}\right\} \mathrm{NMR}$ spectrum of $\left(S_{\mathrm{P}}, S_{\mathrm{P}}, S_{\mathrm{C}}, S_{\mathrm{C}}\right)-6\left(75 \mathrm{MHz}, \mathrm{CD}_{2} \mathrm{Cl}_{2}\right)$.

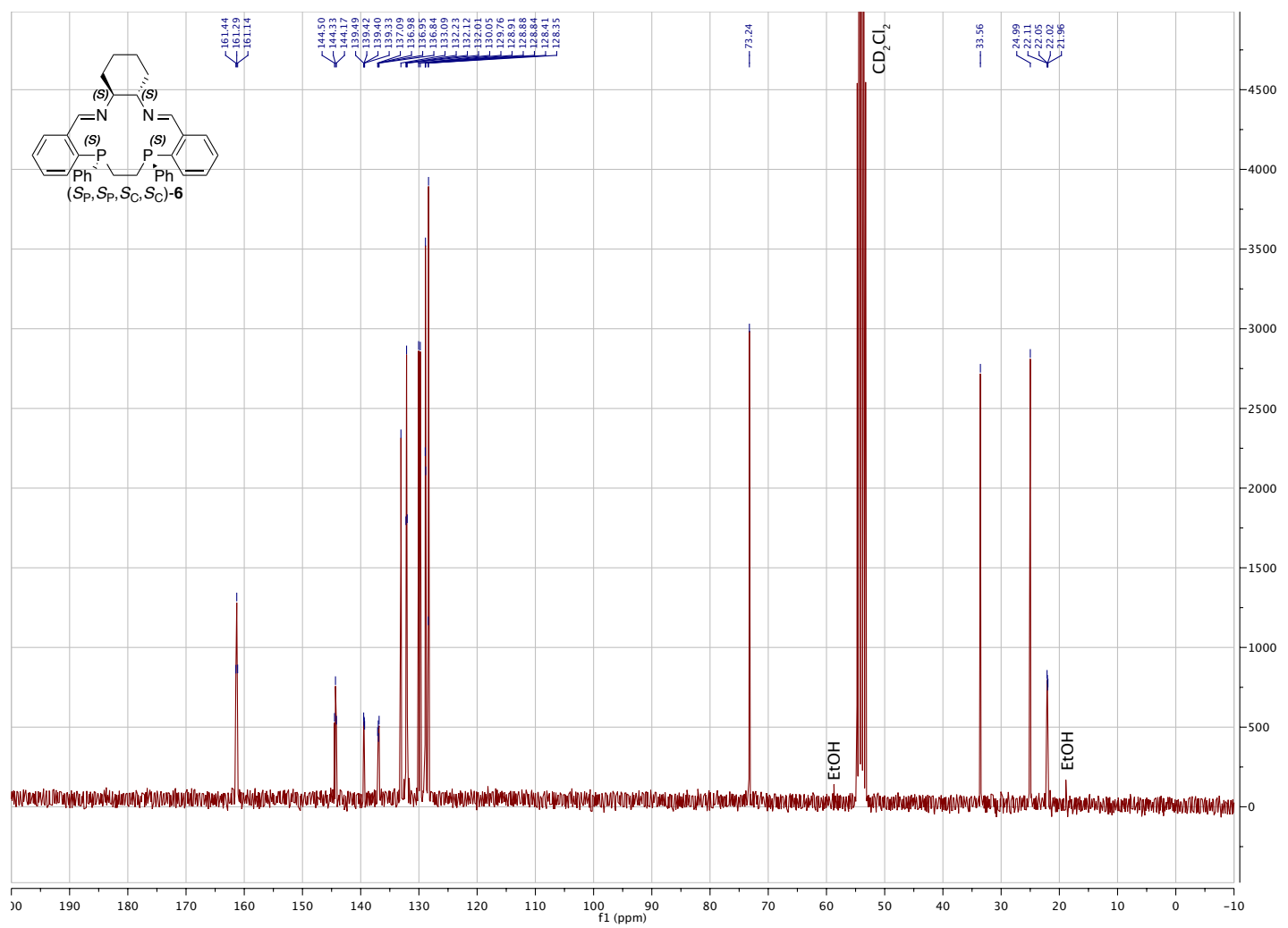




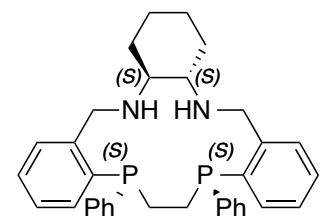

$\left(S_{\mathrm{P}}, S_{\mathrm{P}}, S_{\mathrm{C}}, S_{\mathrm{C}}\right)-7$

Synthesis of $(5 S, 8 S, 14 \mathrm{a} S, 18 \mathrm{a} S)-5,8-D i p h e n y l-5,6,7,8,13,14,14 a, 15,16,17$, 18,18a,19,20-tetradecahydrotribenzo $[b, f, l][1,4]$ diaza $[8,11]$ diphosphacyclotetradecine, $\quad\left(S_{\mathrm{P},} S_{\mathrm{P}}\right.$, $\boldsymbol{S}_{\mathrm{C},} \boldsymbol{S}_{\mathrm{C}}$ )-7. The title compound was prepared following a literature procedure. ${ }^{\mathrm{S}}$ A flame-dried $250 \mathrm{~mL}$ Schlenk flask was charged with lithium aluminum hydride (2.07 g, $54.6 \mathrm{mmol}, 10$ equiv) and THF $(50 \mathrm{~mL})$, and the solution was cooled to $0{ }^{\circ} \mathrm{C}$. After $10 \mathrm{~min},\left(S_{\mathrm{P}}, S_{\mathrm{P}}, S_{\mathrm{C}}, S_{\mathrm{C}}\right)-6(2.91 \mathrm{~g}, 5.46$ mmol) was added, and the solids were washed down with THF ( $5 \mathrm{~mL})$. The solution was warmed to room temperature overnight to afford an orange solution. The flask was cooled to $0{ }^{\circ} \mathrm{C}$, and EtOAc (15 mL; degassed by bubbling nitrogen through for $1 \mathrm{~h}$ ) was carefully introduced (Note: Exothermic reaction). After stirring the solution at room temperature for $2 \mathrm{~h}$, a $2: 1$ weight mixture of $\mathrm{Na}_{2} \mathrm{SO}_{4} /$ Celite $^{\circledR}$ (24 g) was added, followed by water $(7.5 \mathrm{~mL}$; degassed by bubbling nitrogen through for $1 \mathrm{~h}$ ), and then the suspension was vigorously stirred at room temperature. After $2.5 \mathrm{~h}$, the suspension was rapidly filtered in air through a pad of a $2: 1$ weight mixture of $\mathrm{Na}_{2} \mathrm{SO}_{4} /$ Celite ${ }^{\circledR}$ on a glass frit into a $500 \mathrm{~mL}$ two-neck round-bottom flask and washed thoroughly with THF $(350 \mathrm{~mL})$. The solvent was removed using an external cooling trap. The crude product was dissolved in chloroform (6 mL; degassed by bubbling nitrogen through for $1 \mathrm{~h}$ ) and then filtered with EtOAc : $\mathrm{NEt}_{3}(600 \mathrm{~mL}, 19: 1$, degassed by bubbling nitrogen through for $1 \mathrm{~h}$ ) through silica gel $(70 \mathrm{~g})$ in a column under nitrogen. The solvent was rapidly removed at the rotary evaporator $\left(40^{\circ} \mathrm{C}, 20\right.$ mbar) to afford the title product as a white solid, which was used without further purification. Yield: $2.74 \mathrm{~g}(93 \%) .{ }^{1} \mathbf{H}$ NMR $\left(300 \mathrm{MHz}, \mathrm{CD}_{2} \mathrm{Cl}_{2}\right): \delta 7.45-7.39(m, 2 \mathrm{H}, \mathrm{Ar}-$ $H), 7.37-7.32(m, 4 \mathrm{H}, \mathrm{Ar}-H), 7.29-7.17(m, 8 \mathrm{H}, \mathrm{Ar}-H), 7.15-7.07(m, 4 \mathrm{H}, \mathrm{Ar}-H), 4.04\left(d,{ }^{2} J_{\mathrm{H}, \mathrm{H}}\right.$, $=10.5 \mathrm{~Hz}, 2 \mathrm{H}, \mathrm{NCHH}), 3.84\left(d,{ }^{2} J_{\mathrm{H}, \mathrm{H}}=10.5 \mathrm{~Hz}, 2 \mathrm{H}, \mathrm{NCHH}\right), 2.65-2.47(m, 2 \mathrm{H}, \mathrm{NCH}), 2.43-$ $2.16(m, 8 \mathrm{H}, \mathrm{PCHH}(4 \mathrm{H})+\mathrm{CHH}(2 \mathrm{H})+\mathrm{NH}(2 \mathrm{H})), 1.81-1.70(m, 2 \mathrm{H}, \mathrm{CHH}), 1.37-1.10(m, 4 \mathrm{H}$, $\mathrm{CHH}) .{ }^{31} \mathbf{P}\left\{{ }^{1} \mathbf{H}\right\} \mathbf{N M R}\left(122 \mathrm{MHz}, \mathrm{CD}_{2} \mathrm{Cl}_{2}\right): \delta-30.3(s)$. Analytical data are in agreement with literature data. ${ }^{\mathrm{S} 3}$ 
Figure S37: ${ }^{1} \mathrm{H}$ NMR spectrum of $\left(S_{\mathrm{P}}, S_{\mathrm{P}}, S_{\mathrm{C}}, S_{\mathrm{C}}\right)-7\left(300 \mathrm{MHz}, \mathrm{CD}_{2} \mathrm{Cl}_{2}\right)$.

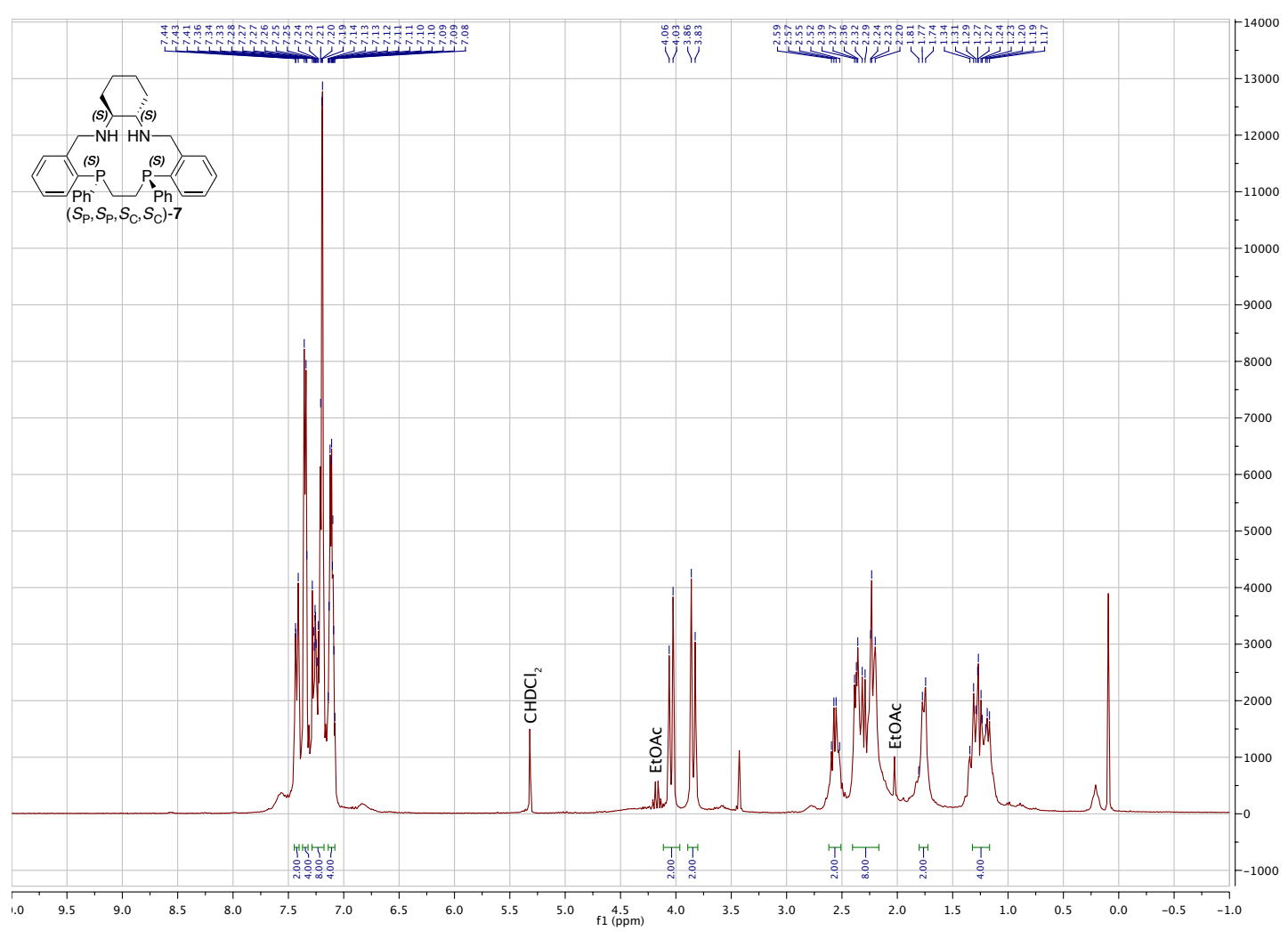

Figure S38: ${ }^{31} \mathrm{P}\left\{{ }^{1} \mathrm{H}\right\}$ NMR spectrum of $\left(S_{\mathrm{P}}, S_{\mathrm{P}}, S_{\mathrm{C}}, S_{\mathrm{C}}\right)-7\left(122 \mathrm{MHz}, \mathrm{CD}_{2} \mathrm{Cl}_{2}\right)$.

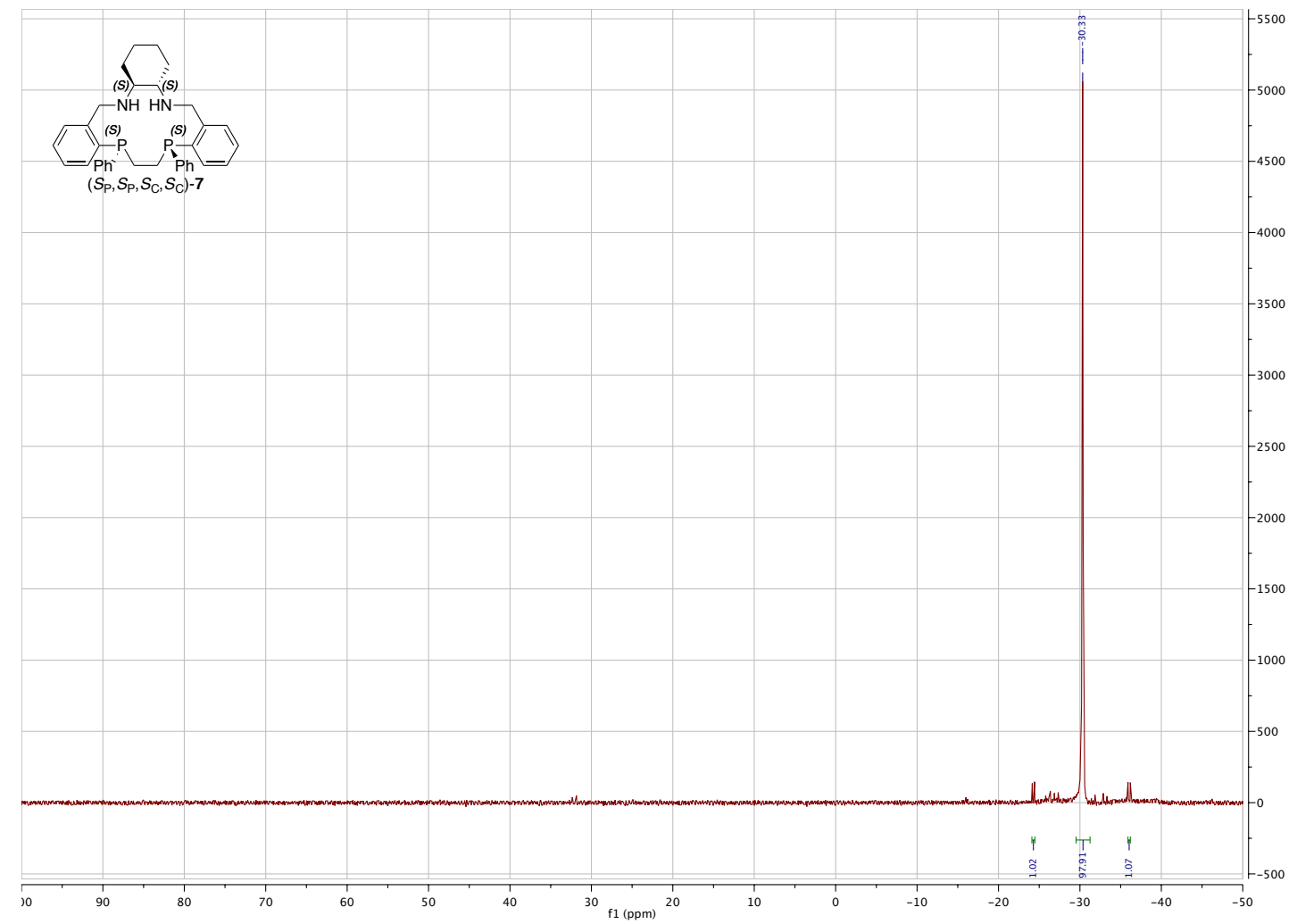




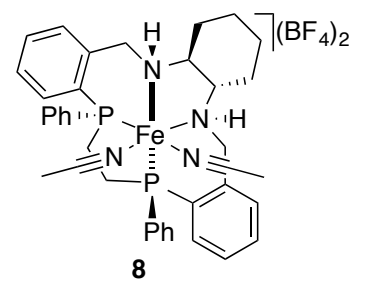

Synthesis of $\left[\mathrm{Fe}(\mathrm{MeCN})_{2}\left(\left(S_{\mathrm{P}}, S_{\mathrm{P}}, S_{\mathrm{C}}, S_{\mathrm{C}}\right)-7\right)\right]\left(\mathrm{BF}_{4}\right)_{2}, 8$. This compound was

prepared following a literature procedure. ${ }^{\mathrm{S} 4}$ A $500 \mathrm{~mL}$ two-neck round-bottom flask containing $\left(S_{\mathrm{P}}, S_{\mathrm{P}}, S_{\mathrm{C}}, S_{\mathrm{C}}\right)-7\left(2.74 \mathrm{~g}, 5.11 \mathrm{mmol}, 1.05\right.$ equiv) was connected to a reflux condenser and $\mathrm{CH}_{2} \mathrm{Cl}_{2}$ ( $65 \mathrm{~mL}$; degassed by 10 vacuum / argon cycles) and $\mathrm{MeCN}$ (30 mL; degassed by 10 vacuum / argon cycles) were added. A separate flame-dried $100 \mathrm{~mL}$ Schlenk flask was charged with iron(II) tetrafluoroborate hexahydrate $(1.63 \mathrm{~g}, 4.86 \mathrm{mmol})$ and $\mathrm{MeCN}(60 \mathrm{~mL}$; degassed by 10 vacuum / argon cycles) and the resulting solution was transferred to the round-bottom flask using a cannula to give a red solution. The Schlenk flask was rinsed twice with $\mathrm{MeCN}(2 \times 20 \mathrm{~mL}$; degassed by 10 vacuum / argon cycles), and the solution was heated in an oil bath at $55^{\circ} \mathrm{C}$. After $1 \mathrm{~h}, \mathrm{DBU}(25 \mu \mathrm{L}, 167 \mu \mathrm{L}$, 3.4 mol\%) was added, and the reaction solution was stirred for $0.75 \mathrm{~h}$. This process was repeated until the ${ }^{31} \mathrm{P}\left\{{ }^{1} \mathrm{H}\right\}$ NMR spectrum of the reaction mixture started to show the isomerization process to the preferred cis- $\beta$ isomer (Note: 5-10 additions of DBU are necessary). After stirring for $2.0 \mathrm{~h}$, the ${ }^{31} \mathrm{P}\left\{{ }^{1} \mathrm{H}\right\}$ NMR spectra showed full conversion to the desired isomer. Then, the solution was filtered with $\mathrm{MeCN}(3 \times 20 \mathrm{~mL}$; degassed by 10 vacuum / argon cycles $)$ through a short pad of Celite $^{\circledast}$ in a Young filter into a flame-dried $500 \mathrm{~mL}$ two-neck round-bottom flask, and the solvent was removed using an external cooling trap. The product was dissolved in $\mathrm{MeCN}(25 \mathrm{~mL}$; degassed by 10 vacuum / argon cycles) and filtered into a flame-dried $150 \mathrm{~mL}$ Schlenk using a cannula filter. The solution was cooled to $-20{ }^{\circ} \mathrm{C}$ and carefully layered with $\mathrm{MeCN}$ ( $5 \mathrm{~mL}$; degassed by 10 vacuum / argon cycles) and $\mathrm{Et}_{2} \mathrm{O}(120 \mathrm{~mL})$. After 5 days, the supernatant was removed to afford the product as orange-red crystals. Yield: $2.73 \mathrm{~g}$ (66\%, single isomer). ${ }^{1} \mathbf{H}$ NMR (300 MHz, $\left.\mathrm{CD}_{2} \mathrm{Cl}_{2}\right)$ : $\delta 7.95\left(t,{ }^{3} J_{\mathrm{H}, \mathrm{H}^{\prime}}=8.1 \mathrm{~Hz}, 1 \mathrm{H}, \mathrm{Ar}-H\right), 7.75\left(t,{ }^{3} J_{\mathrm{H}, \mathrm{H}^{\prime}}=7.5 \mathrm{~Hz}, 1 \mathrm{H}, \mathrm{Ar}-H\right), 7.68-7.44(m, 8 \mathrm{H}, \mathrm{Ar}-H)$, 
$7.39-7.23(m, 3 \mathrm{H}, \operatorname{Ar}-H), 7.13\left(d d,{ }^{3} J_{\mathrm{H}, \mathrm{H}^{\prime}}=7.1 \mathrm{~Hz},{ }^{4} J_{\mathrm{H}, \mathrm{H}^{\prime}}=4.4 \mathrm{~Hz}, 1 \mathrm{H}, \mathrm{Ar}-H\right), 7.05-6.94(m, 2 \mathrm{H}$, $\operatorname{Ar}-H), 6.28\left(t,{ }^{3} J_{\mathrm{H}, \mathrm{H}^{\prime}}=8.5 \mathrm{~Hz}, 2 \mathrm{H}, \operatorname{Ar}-H\right), 6.07\left(d,{ }^{3} J_{\mathrm{H}, \mathrm{H}^{\prime}}=11.6 \mathrm{~Hz}, 1 \mathrm{H}, \mathrm{N} H\right), 4.84-4.71(m, 1 \mathrm{H}$, $\mathrm{NCHH}), 4.59\left(d,{ }^{2} J_{\mathrm{H}, \mathrm{H}^{\prime}}=17.5 \mathrm{~Hz}, 1 \mathrm{H}, \mathrm{NCHH}\right), 3.70\left(d d,{ }^{2} J_{\mathrm{H}, \mathrm{H}^{\prime}}=13.4 \mathrm{~Hz},{ }^{3} J_{\mathrm{H}, \mathrm{H}^{\prime}}=11.0 \mathrm{~Hz}, 1 \mathrm{H}\right.$, $\mathrm{NCHH}), 3.55\left(d,{ }^{2} J_{\mathrm{H}, \mathrm{H}}=13.4 \mathrm{~Hz}, 1 \mathrm{H}, \mathrm{NCHH}\right), 3.51-3.29(m, 2 \mathrm{H}, \mathrm{PC} H \mathrm{H}), 3.17-2.92(m, 2 \mathrm{H}$, $\mathrm{PCHH}), 2.65\left(\mathrm{br} d,{ }^{3} J_{\mathrm{H}, \mathrm{H}^{\prime}}=12.4 \mathrm{~Hz}, 1 \mathrm{H}, \mathrm{CH}\right), 2.37-2.15(m, 2 \mathrm{H}, \mathrm{NCH}+\mathrm{CH}), 2.14(s, 3 \mathrm{H}$, $\left.\mathrm{CH}_{3} \mathrm{CN}\right), 2.11\left(s, 3 \mathrm{H}, \mathrm{CH}_{3} \mathrm{CN}\right), 1.92-1.74(m, 2 \mathrm{H}, \mathrm{NCH}+\mathrm{CHH}), 1.67-1.48(m, 2 \mathrm{H}, \mathrm{CHH}), 1.30$ $-1.10(m, 1 \mathrm{H}, \mathrm{CHH}), 0.96-0.77(m, 1 \mathrm{H}, \mathrm{CHH}), 0.01--0.13(m, 1 \mathrm{H}, \mathrm{NH}),-0.13--0.28(m, 1 \mathrm{H}$, CHH). ${ }^{31} \mathbf{P}\left\{{ }^{1} \mathbf{H}\right\}$ NMR $\left(122 \mathrm{MHz}, \mathrm{CD}_{2} \mathrm{Cl}_{2}\right): \delta 103.4\left(d,{ }^{2} J_{\mathrm{P}, \mathrm{P}},=19.7 \mathrm{~Hz}\right), 92.3\left(d,{ }^{2} J_{\mathrm{P}, \mathrm{P}},=19.7 \mathrm{~Hz}\right)$. ${ }^{13} \mathbf{C}\left\{{ }^{31} \mathbf{P},{ }^{1} \mathbf{H}\right\}$ NMR (126 MHz, $\mathrm{CD}_{2} \mathrm{Cl}_{2}$ ): $\delta 142.5$ (arom.), 142.0 (arom.), 136.1 (arom), 133.51 (arom.), 133.45 (arom.), 133.3 (arom.), 132.9 (arom.), 132.4 (arom.), 132.04 (arom.), 132.00 (arom.), 131.8 (arom.), 131.74 (arom.), 131.66 (arom), 131.61 (arom.), 131.56 (arom.), 131.4 (arom.), 131.3 (arom.), 130.2 (arom.), 129.9 (arom.), 129.4 (arom.), $127.5\left(\mathrm{CH}_{3} \mathrm{CN}\right), 124.1$ $\left(\mathrm{CH}_{3} \mathrm{CN}\right), 66.0(\mathrm{NCH}), 65.4(\mathrm{NCH}), 53.5\left(\mathrm{NCH}_{2}\right), 50.2\left(\mathrm{NCH}_{2}\right), 31.3\left(\mathrm{CH}_{2}\right), 30.2\left(\mathrm{CH}_{2}\right), 27.4$ $\left(\mathrm{PCH}_{2}\right), 27.2\left(\mathrm{PCH}_{2}\right), 25.0\left(\mathrm{CH}_{2}\right), 24.7\left(\mathrm{CH}_{2}\right), 5.1\left(\mathrm{CH}_{3} \mathrm{CN}\right), 4.3\left(\mathrm{CH}_{3} \mathrm{CN}\right) .{ }^{11} \mathbf{B}$ NMR $(96 \mathrm{MHz}$, $\mathrm{CD}_{2} \mathrm{Cl}_{2}$ ): $\delta 0.0(s)$. IR (liquid film, $\left.\mathrm{cm}^{-1}\right): 3228(\mathrm{~N}-\mathrm{H}), 3061(\mathrm{C}-\mathrm{H}), 2939(\mathrm{C}-\mathrm{H}), 2861(\mathrm{C}-\mathrm{H}), 1630$, $1591,1481,1450,1434,1387,1371,1320,1267,1243,1194,1168,1050,1034$. IR $\left(\mathrm{KBr}, \mathrm{cm}^{-1}\right)$ : $2319(\mathrm{MeCN}), 2285$ (MeCN), 2272 (MeCN). HRMS (MALDI): Calcd. for $\mathrm{C}_{34} \mathrm{H}_{38} \mathrm{FFeN}_{2} \mathrm{P}_{2} \mathrm{~m} / \mathrm{z}$ 611.1839, found $m / z 611.1835\left[\mathrm{FeF}\left(\left(S_{\mathrm{P}}, S_{\mathrm{P}}, S_{\mathrm{C}}, S_{\mathrm{C}}\right)-7\right)\right]^{+}$. EA: Calcd. for $\mathrm{C}_{38} \mathrm{H}_{44} \mathrm{~B}_{2} \mathrm{~F}_{8} \mathrm{FeN}_{4} \mathrm{P}_{2}: \mathrm{C}, 53.81$; $\mathrm{H}, 5.23 ; \mathrm{N}, 6.61$; found: $\mathrm{C}, 54.03 ; \mathrm{H}, 5.32 ; \mathrm{N}, 6.75$. Analytical data are in agreement with literature data. ${ }^{\mathrm{S} 3,4}$ 
Figure S39: ${ }^{1} \mathrm{H} \mathrm{NMR}$ spectrum of $8\left(300 \mathrm{MHz}, \mathrm{CD}_{2} \mathrm{Cl}_{2}\right)$.

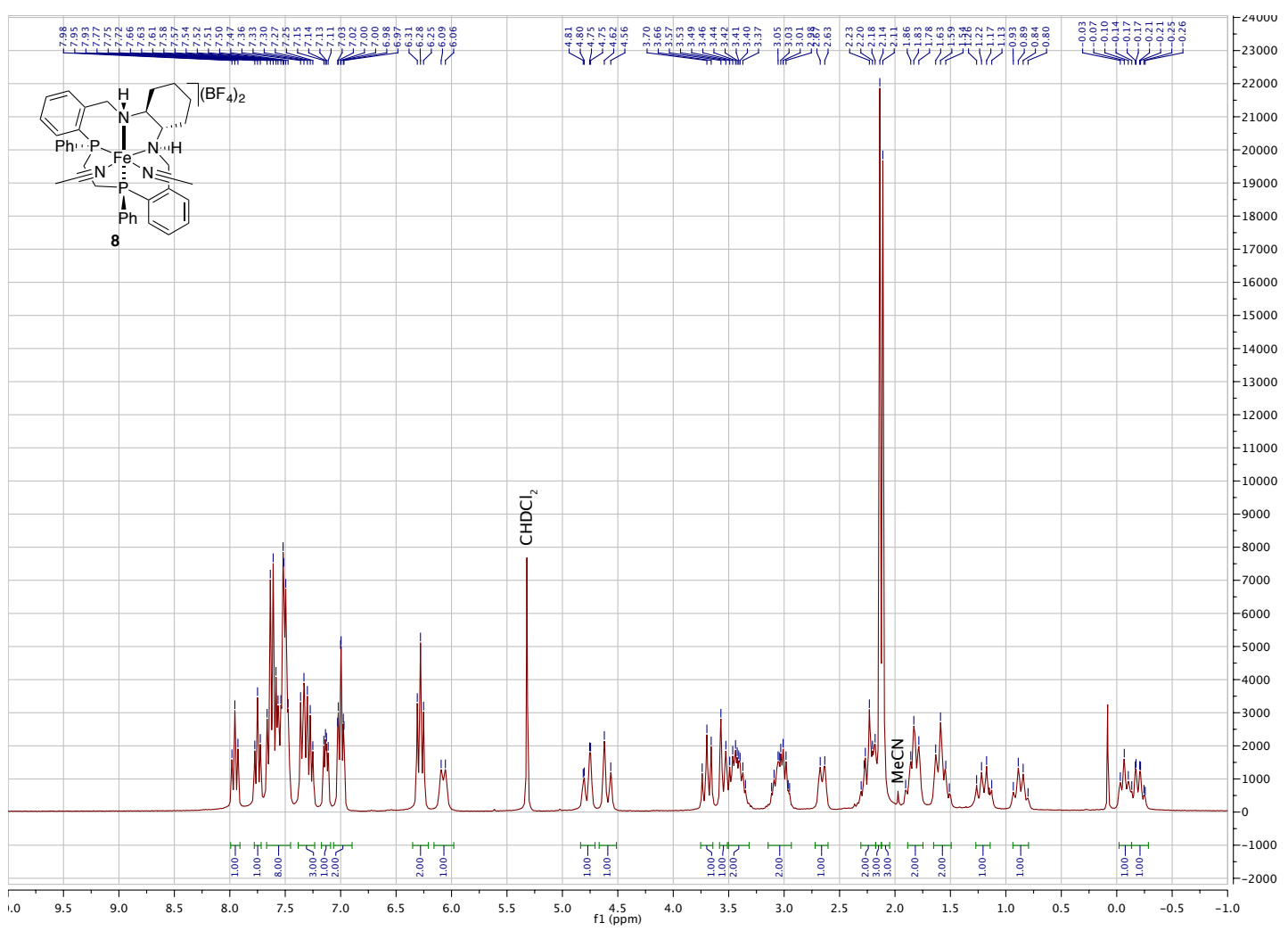

Figure S40: ${ }^{31} \mathrm{P}\left\{{ }^{1} \mathrm{H}\right\}$ NMR spectrum of $8\left(122 \mathrm{MHz}, \mathrm{CD}_{2} \mathrm{Cl}_{2}\right)$.

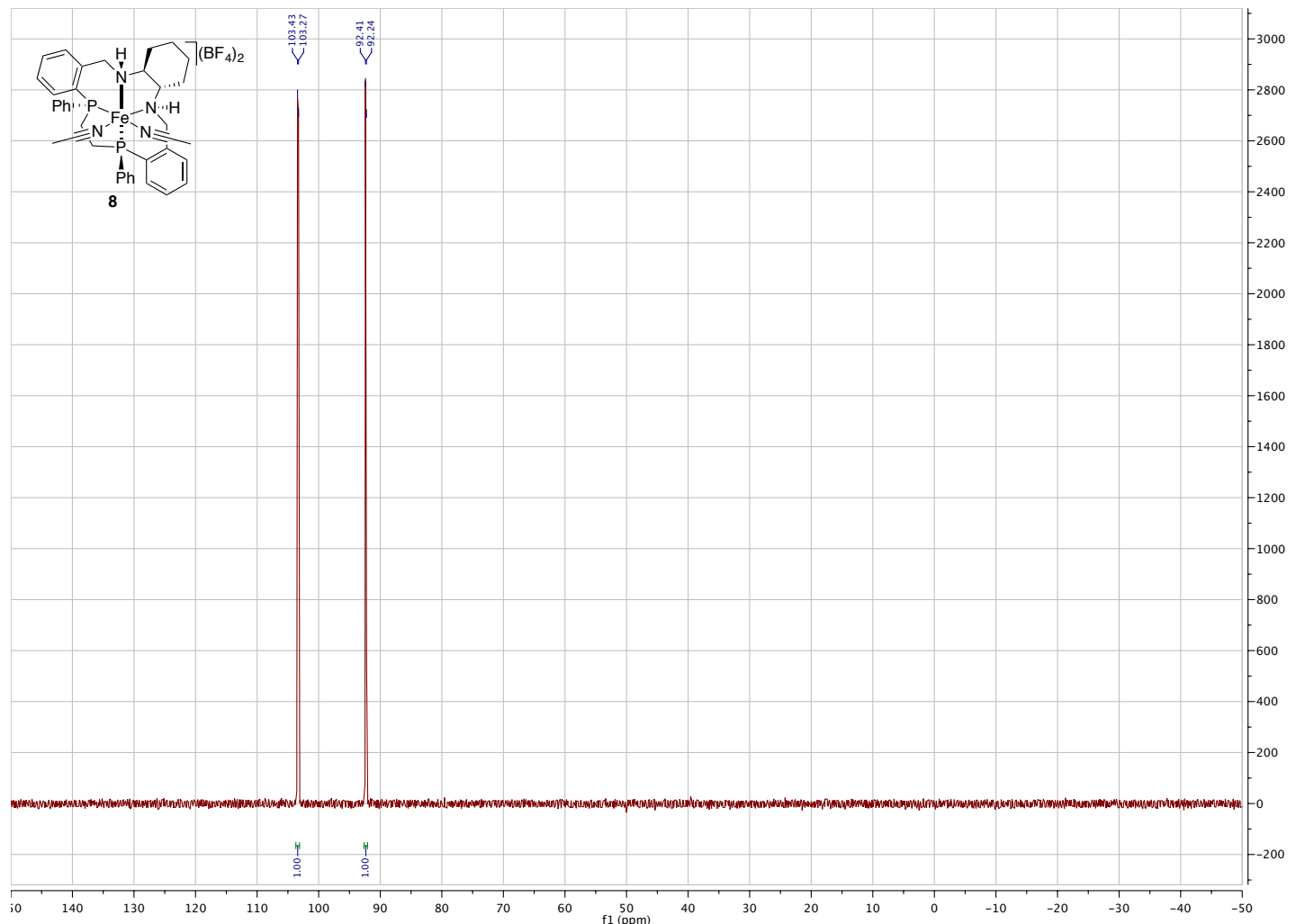


Figure S41: ${ }^{13} \mathrm{C}\left\{{ }^{31} \mathrm{P},{ }_{1}^{1} \mathrm{H}\right\}$ NMR spectrum of $8\left(126 \mathrm{MHz}, \mathrm{CD}_{2} \mathrm{Cl}_{2}\right)$.

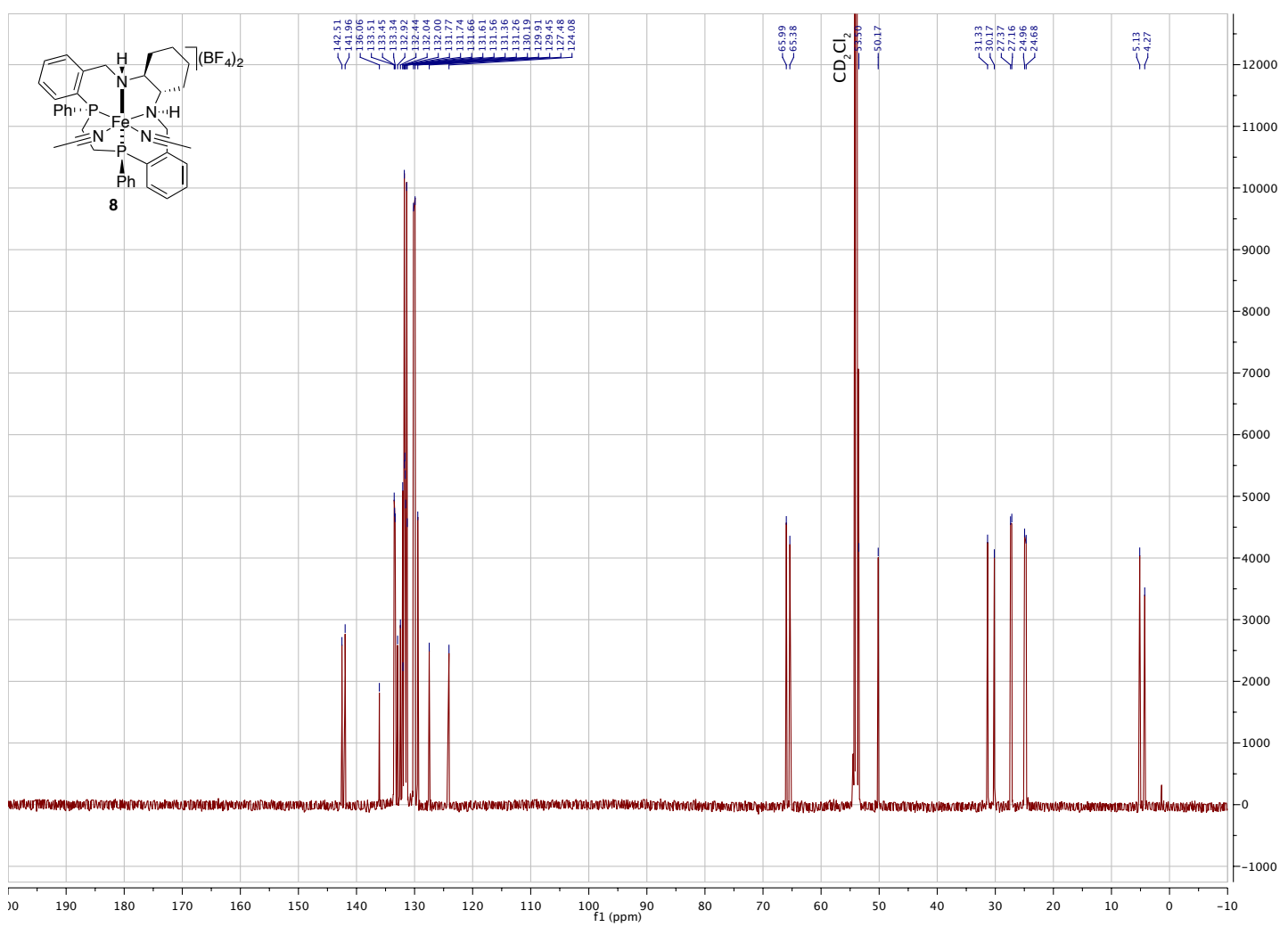

Figure S42: ${ }^{11} \mathrm{~B}$ NMR spectrum of $8\left(96 \mathrm{MHz}, \mathrm{CD}_{2} \mathrm{Cl}_{2}\right)$.

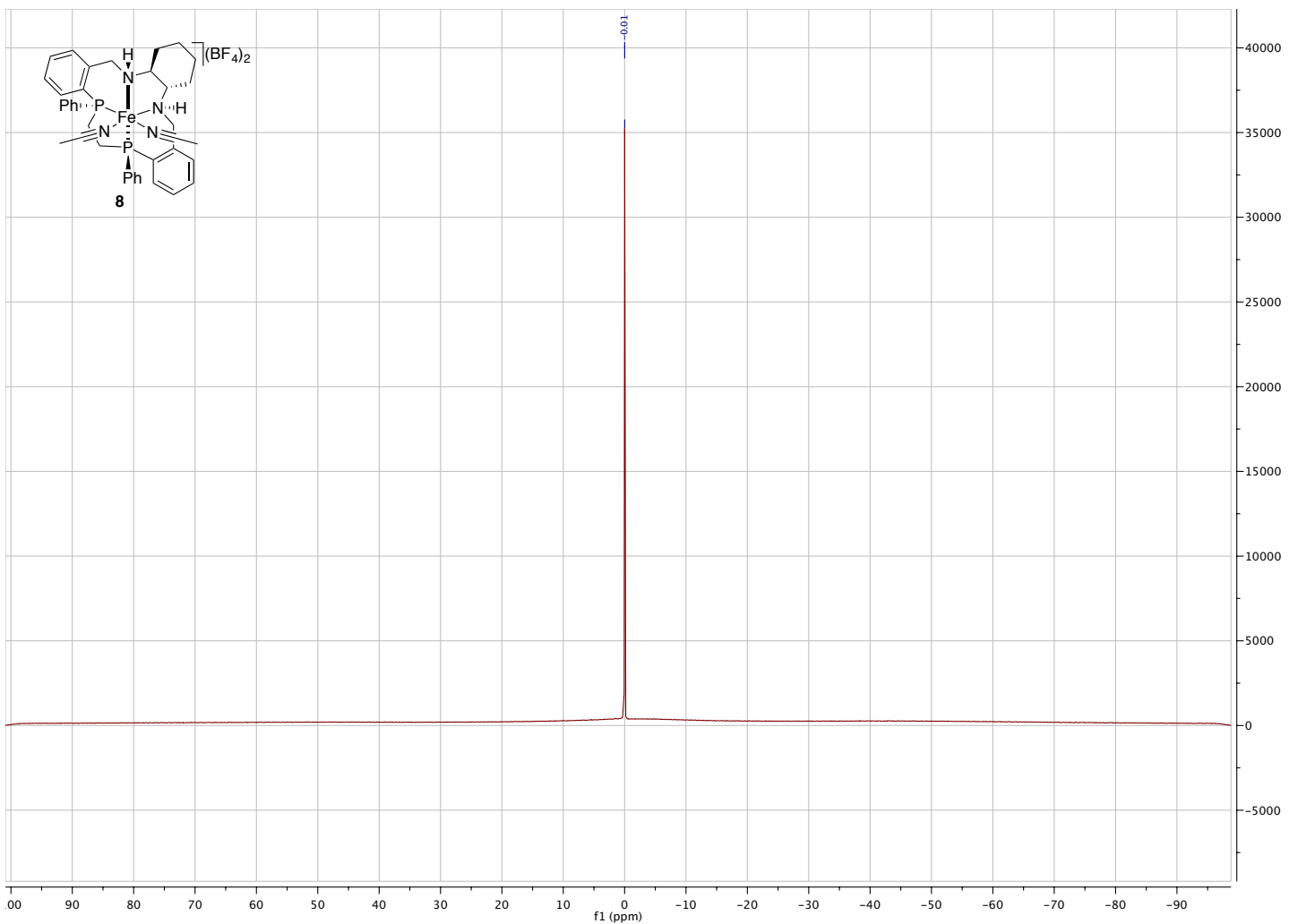


Figure S43: ${ }^{31} \mathrm{P}\left\{{ }^{1} \mathrm{H}\right\}$ NMR spectrum initial complexation mixture $\left(122 \mathrm{MHz}, \mathrm{MeCN} / \mathrm{CH}_{2} \mathrm{Cl}_{2}\right)$.
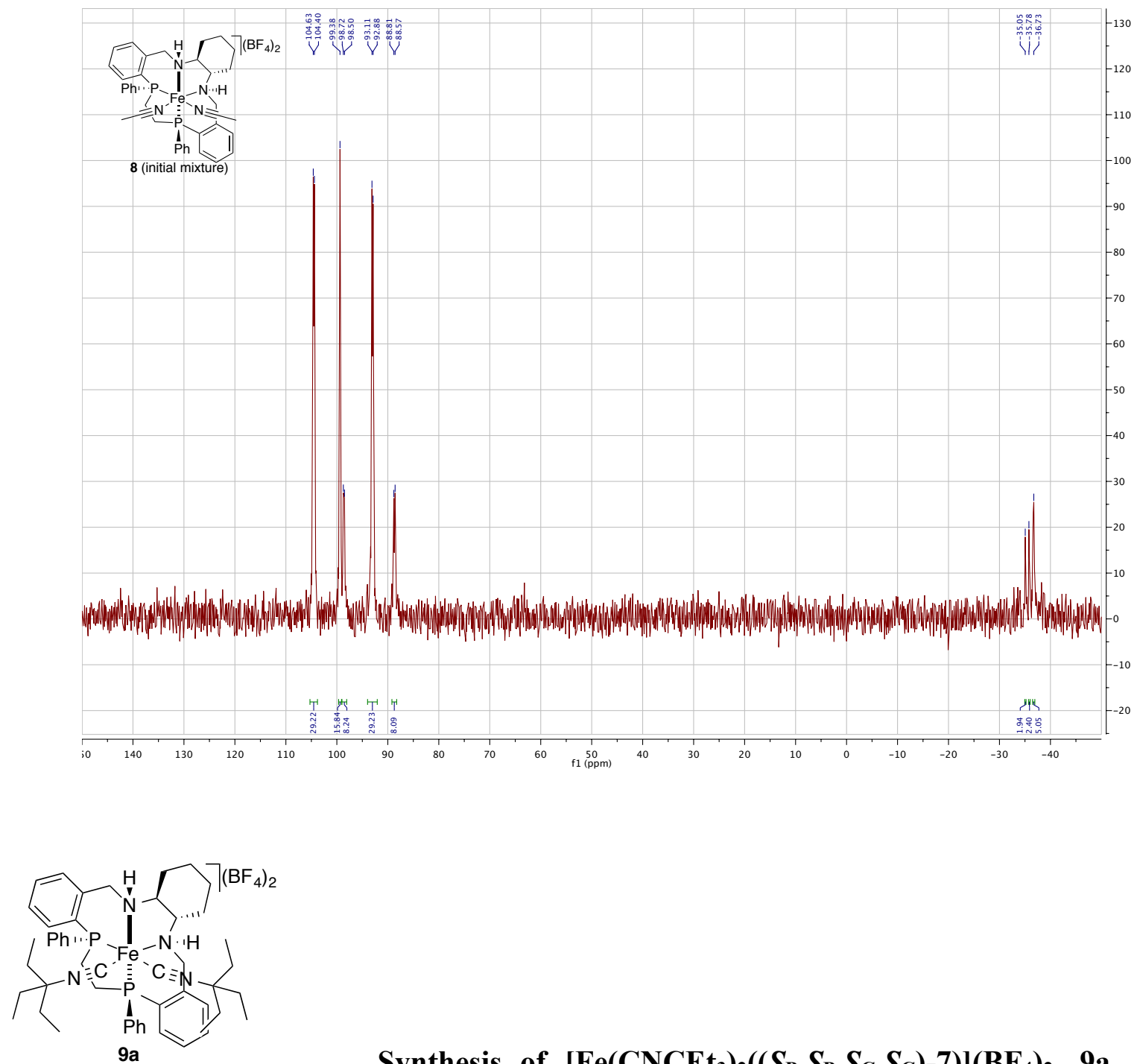

Synthesis of $\left[\mathrm{Fe}\left(\mathrm{CNCEt}_{3}\right)_{2}\left(\left(\boldsymbol{S}_{\mathrm{P}}, S_{\mathrm{P}}, S_{\mathrm{C}}, S_{\mathrm{C}}\right)-7\right)\right]\left(\mathrm{BF}_{4}\right)_{2}$, 9a. The title compound was prepared following a literature procedure. ${ }^{\mathrm{S} 4}$ A flame-dried $50 \mathrm{~mL}$ Young flask was charged with 8 (1.000 g, $1.18 \mathrm{mmol}), \mathrm{CH}_{2} \mathrm{Cl}_{2}$ (12 mL; degassed by 10 vacuum / argon cycles), and 3-ethyl-3-isocyanopentane $\mathrm{S}^{\mathrm{S}}(0.738 \mathrm{~g}, 5.89 \mathrm{mmol}, 5.0$ equiv), and the solution was stirred for $24 \mathrm{~h}$ at $50{ }^{\circ} \mathrm{C}$ (Note: 3 -Ethyl-3-isocyanopentane was weighted and added using a syringe). The solution was cooled to room temperature and filtered into a flame-dried $100 \mathrm{~mL}$ Schlenk flask using a cannula filter. The Young flask was rinsed twice with $\mathrm{CH}_{2} \mathrm{Cl}_{2}(2 \times 5 \mathrm{~mL}$; degassed by 10 vacuum / argon cycles), and the solvent was removed using an external cooling trap. The crude product was dissolved in $\mathrm{CH}_{2} \mathrm{Cl}_{2}$ (10 mL; degassed by 10 vacuum / argon cycles) and layered with hexane 
$(80 \mathrm{~mL})$. After 2 days, an orange oil had separated, and the supernatant was removed using a cannula. This process was repeated once to afford the title product as a yellow solid after drying under high vacuum. Yield: $1.186 \mathrm{~g}\left(99 \%\right.$, single isomer). ${ }^{1} \mathbf{H}$ NMR $\left(300 \mathrm{MHz}, \mathrm{CD}_{2} \mathrm{Cl}_{2}\right): \delta 7.98-$ $7.82(m, 2 \mathrm{H}, \mathrm{Ar}-H), 7.80-7.63(m, 3 \mathrm{H}, \mathrm{Ar}-H), 7.62-7.51(m, 3 \mathrm{H}, \mathrm{Ar}-H), 7.46\left(t d,{ }^{3} J_{\mathrm{H}, \mathrm{H}^{\prime}}=7.6 \mathrm{~Hz}\right.$, $\left.{ }^{4} J_{\mathrm{H}, \mathrm{H}^{\prime}}=2.5 \mathrm{~Hz}, 2 \mathrm{H}, \mathrm{Ar}-H\right), 7.39-7.19(m, 4 \mathrm{H}, \mathrm{Ar}-H), 7.12-7.01(m, 2 \mathrm{H}, \mathrm{Ar}-H), 6.20\left(t,{ }^{3} J_{\mathrm{H}, \mathrm{H}^{\prime}}=\right.$ $8.7 \mathrm{~Hz}, 2 \mathrm{H}, \mathrm{Ar}-H), 5.74\left(d,{ }^{3} J_{\mathrm{H}, \mathrm{H}^{\prime}}=11.8 \mathrm{~Hz}, 1 \mathrm{H}, \mathrm{NH}\right), 4.72\left(d,{ }^{2} J_{\mathrm{H}, \mathrm{H}^{\prime}}=18.4 \mathrm{~Hz}, 1 \mathrm{H}, \mathrm{NCHH}\right), 4.57$ $\left(d,{ }^{2} J_{\mathrm{H}, \mathrm{H}^{\prime}}=18.4 \mathrm{~Hz}, 1 \mathrm{H}, \mathrm{NCHH}\right), 4.13-3.96(m, 2 \mathrm{H}, \mathrm{NCHH}), 3.73-3.48(m, 1 \mathrm{H}, \mathrm{PCHH}), 3.24-$ $2.79(m, 3 \mathrm{H}, \mathrm{PC} H \mathrm{H}), 2.57-2.43(m, 1 \mathrm{H}, \mathrm{CHH}), 2.41-2.17(m, 2 \mathrm{H}, \mathrm{CHH}+\mathrm{NCH}), 1.90-1.73(m$, $2 \mathrm{H}, \mathrm{NCH}+\mathrm{CHH}), 1.72-1.24(m, 15 \mathrm{H}, \mathrm{N} H(1 \mathrm{H})+\mathrm{C} H \mathrm{H}(2 \mathrm{H})+\mathrm{CNCCH}(12 \mathrm{H})), 1.20-1.01(m$, $1 \mathrm{H}, \mathrm{CH}), 0.86-0.64\left(m, 19 \mathrm{H}, \mathrm{CH} H(1 \mathrm{H})+\mathrm{CH}_{3}(18 \mathrm{H})\right),-0.14--0.32(m, 1 \mathrm{H}, \mathrm{CH})$. ${ }^{31} \mathbf{P}\left\{{ }^{1} \mathbf{H}\right\}$ NMR $\left(122 \mathrm{MHz}, \mathrm{CD}_{2} \mathrm{Cl}_{2}\right): \delta 90.0\left(d,{ }^{2} J_{\mathrm{P}, \mathrm{P}}=19.0 \mathrm{~Hz}\right), 87.3\left(d,{ }^{2} J_{\mathrm{P}, \mathrm{P}},=19.0 \mathrm{~Hz}\right)$. ${ }^{13} \mathbf{C}\left\{{ }^{31} \mathbf{P},{ }^{1} \mathbf{H}\right\}$ NMR (126 MHz, $\left.\mathrm{CD}_{2} \mathrm{Cl}_{2}\right): \delta 157.5$ ( $\left.m, C \mathrm{NCEt}_{3}\right), 154.8$ ( $\left.m, \mathrm{CNCEt}_{3}\right), 142.7$ (arom.), 141.6 (arom.), 134.7 (arom.), 134.2 (arom.), 134.05 (arom.), 133.96 (arom.), 132.6 (arom.), 132.5 (arom.), 132.3 (2C, arom.), 132.2 (arom.), 132.0 (arom.), 131.70 (arom.), 131.66 (arom.), 131.6 (arom.), 130.4 (arom.), 130.1 (arom.), 130.0 (arom.), 125.4 (arom.), 123.8 (arom.), 72.2 (m, $\left.\mathrm{CNCEt}_{3}\right), 71.3\left(m, \mathrm{CNCEt}_{3}\right), 67.3(\mathrm{NCH}), 64.6(\mathrm{NCH}), 55.8\left(\mathrm{NCH}_{2}\right), 51.1\left(\mathrm{NCH}_{2}\right), 31.8\left(\mathrm{CH}_{2}\right)$, 31.4 $\left(\mathrm{PCH}_{2}\right), 30.1\left(\mathrm{CNC}\left(\mathrm{CH}_{2} \mathrm{CH}_{3}\right)_{3}\right), 29.7\left(\mathrm{CNC}\left(\mathrm{CH}_{2} \mathrm{CH}_{3}\right)_{3}\right), 29.6\left(\mathrm{CH}_{2}\right), 27.1\left(\mathrm{PCH}_{2}\right), 24.8\left(\mathrm{CH}_{2}\right)$, $24.6\left(\mathrm{CH}_{2}\right), 8.6\left(\mathrm{CNC}\left(\mathrm{CH}_{2} \mathrm{CH}_{3}\right)_{3}\right), 8.3\left(\mathrm{CNC}\left(\mathrm{CH}_{2} \mathrm{CH}_{3}\right)_{3}\right) .{ }^{11} \mathbf{B} \mathbf{N M R}\left(96 \mathrm{MHz}, \mathrm{CD}_{2} \mathrm{Cl}_{2}\right): \delta 0.0\left(\mathrm{BF}_{4}\right)$. IR (liquid film, cm ${ }^{-1}$ ): 3242 (N-H), 3224 (N-H), 3060 (C-H), 2975 (C-H), 2942 (C-H), 2882 (C-H), 2864 (C-H), 2154 (isonitrile), 2115 (isonitrile), 1632, 1591, 1481, 1456, 1435, 1386, 1337, 1306, 1282, 1267, 1244, 1192, 1150, 1128, 1056. HRMS (MALDI): Calcd. for $\mathrm{C}_{50} \mathrm{H}_{68} \mathrm{BF}_{4} \mathrm{FeN}_{4} \mathrm{P}_{2} \mathrm{~m} / \mathrm{z}$ 929.4302, found $m / z 929.4281\left[\mathrm{M}-\mathrm{BF}_{4}\right]^{+}$. EA: Calcd. for $\mathrm{C}_{50} \mathrm{H}_{68} \mathrm{~B}_{2} \mathrm{~F}_{8} \mathrm{FeN}_{4} \mathrm{P}_{2}$ : C, 59.08; H, 6.74; $\mathrm{N}, 5.51$; found: 59.14; H, 6.96; N, 5.39 . 
Figure S44: ${ }^{1} \mathrm{H}$ NMR spectrum of 9a $\left(300 \mathrm{MHz}, \mathrm{CD}_{2} \mathrm{Cl}_{2}\right)$.

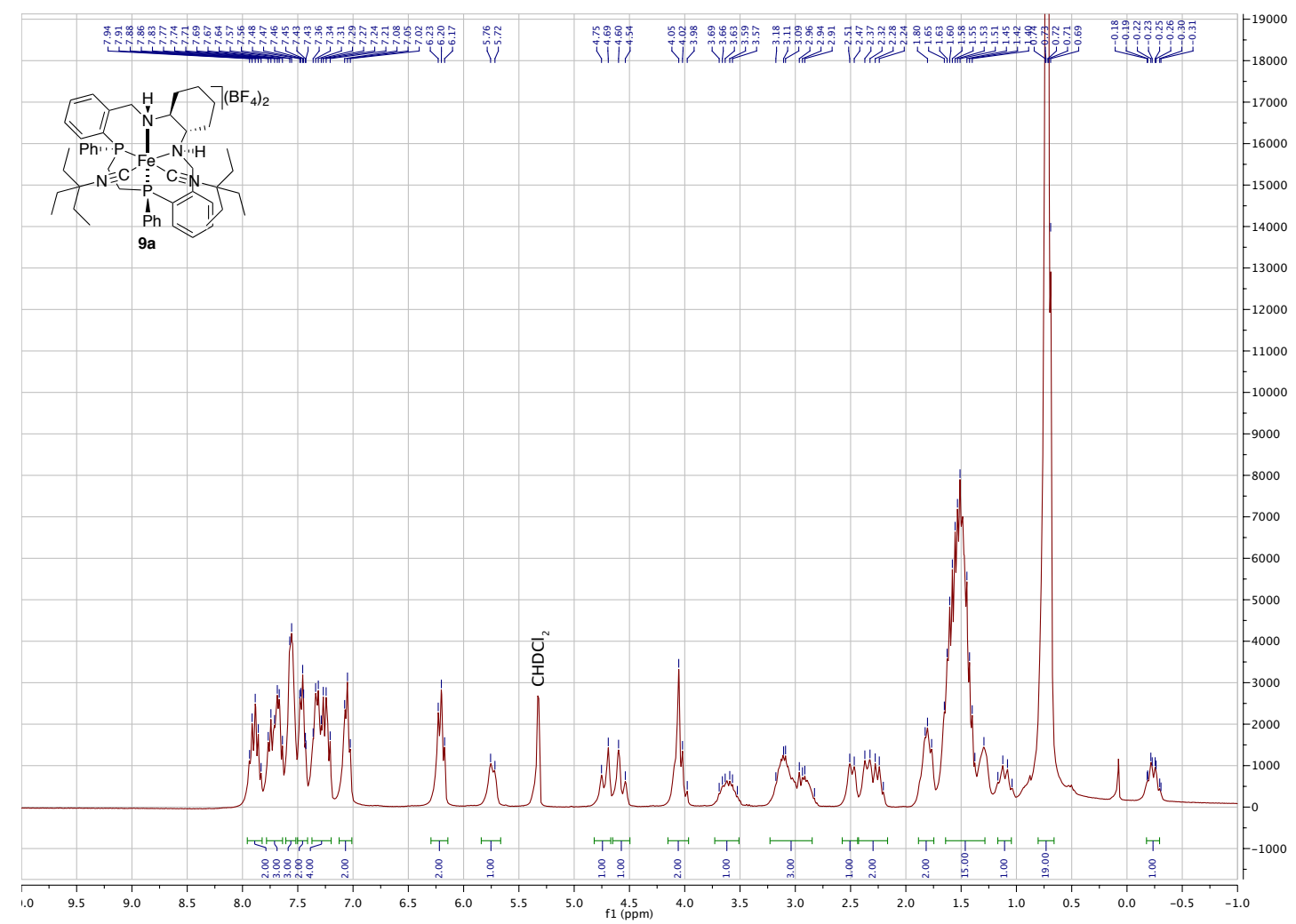

Figure S45: ${ }^{31} \mathrm{P}\left\{{ }^{1} \mathrm{H}\right\}$ NMR spectrum of 9a $\left(122 \mathrm{MHz}, \mathrm{CD}_{2} \mathrm{Cl}_{2}\right)$.

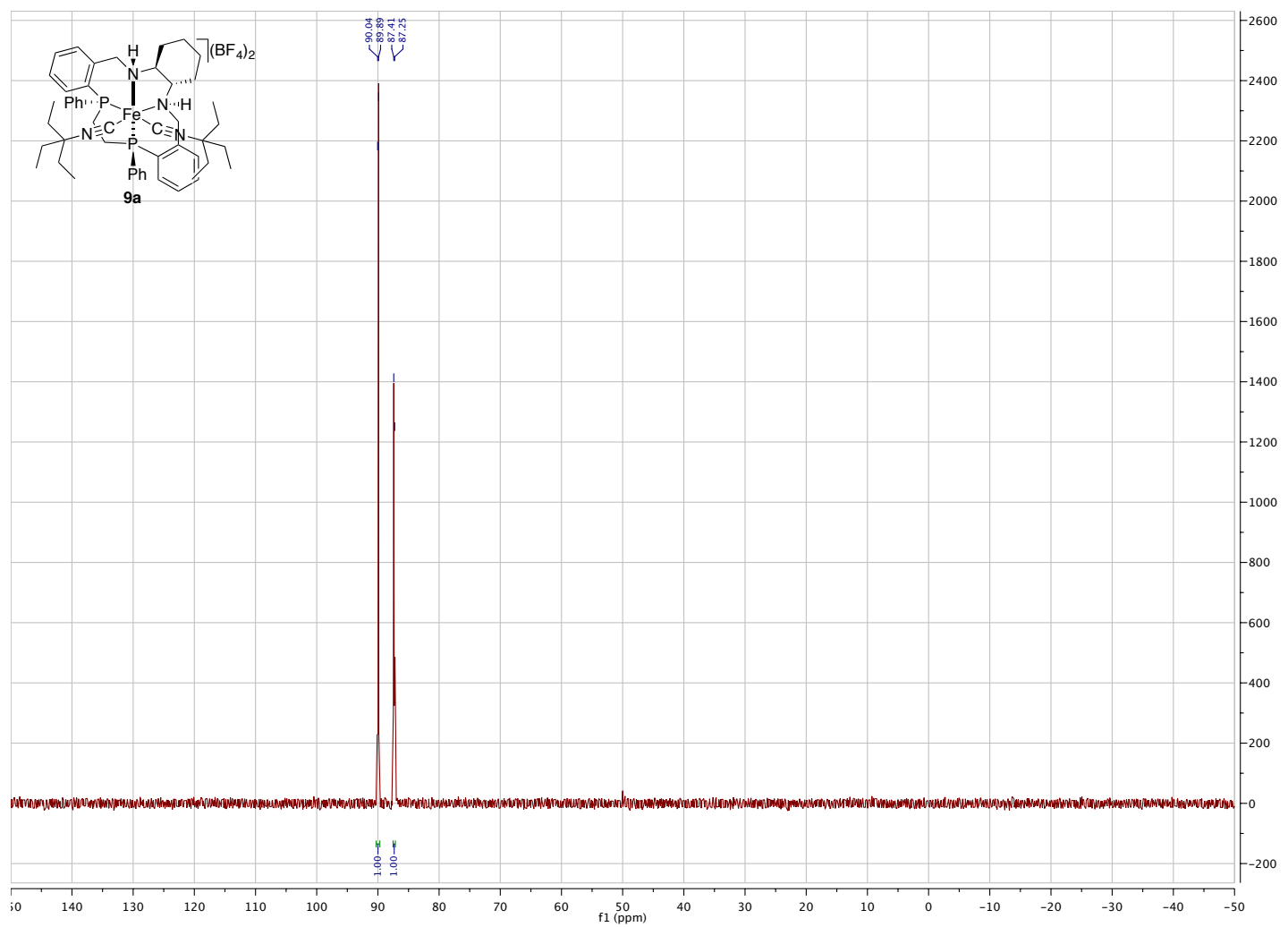


Figure S46: ${ }^{13} \mathrm{C}\left\{{ }^{31} \mathrm{P},{ }^{1} \mathrm{H}\right\} \mathrm{NMR}$ spectrum of 9a $\left(126 \mathrm{MHz}, \mathrm{CD}_{2} \mathrm{Cl}_{2}\right)$.

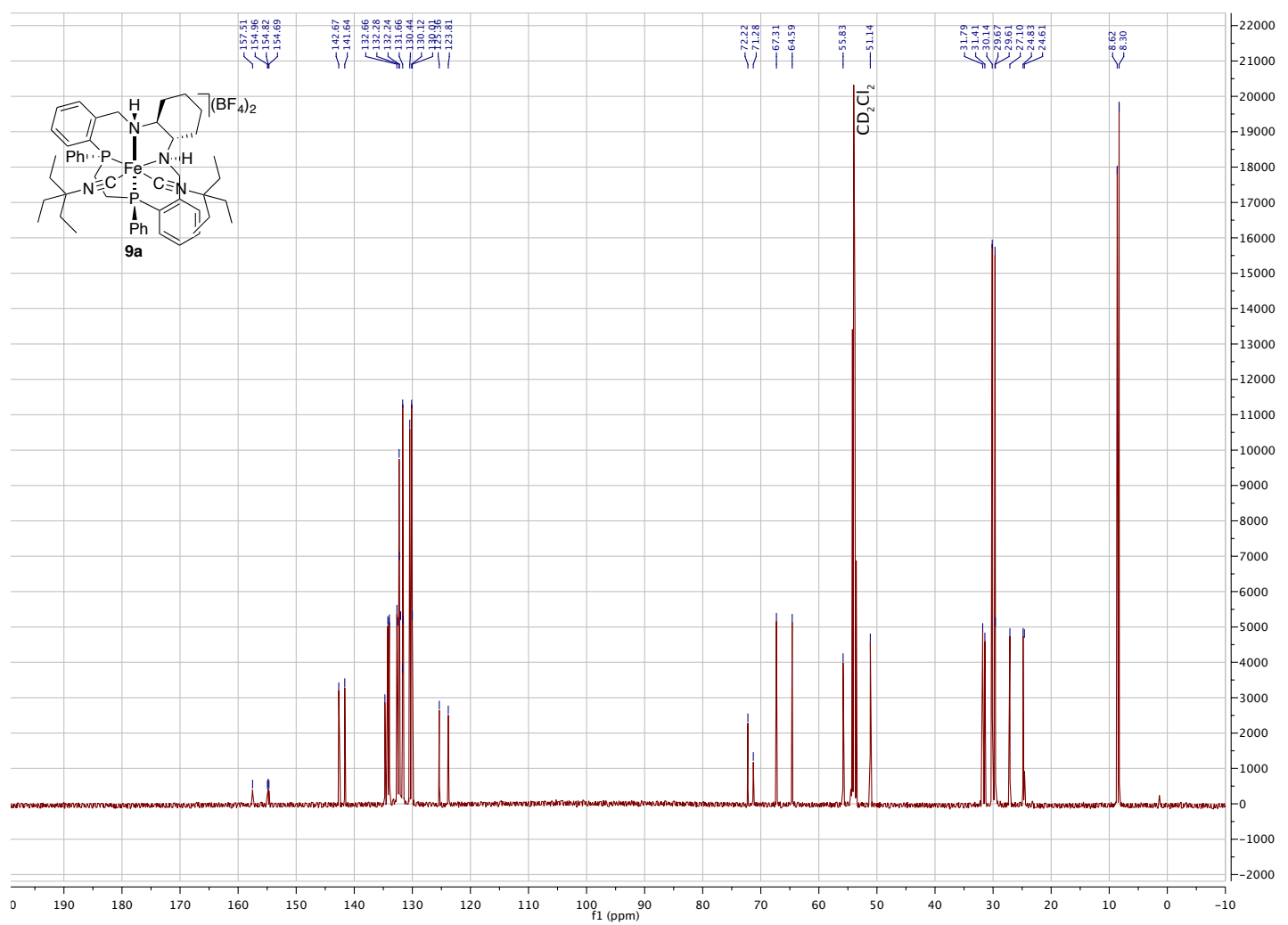

Figure S47: ${ }^{11} \mathrm{~B}$ NMR spectrum of $9 \mathbf{a}\left(96 \mathrm{MHz}, \mathrm{CD}_{2} \mathrm{Cl}_{2}\right)$.

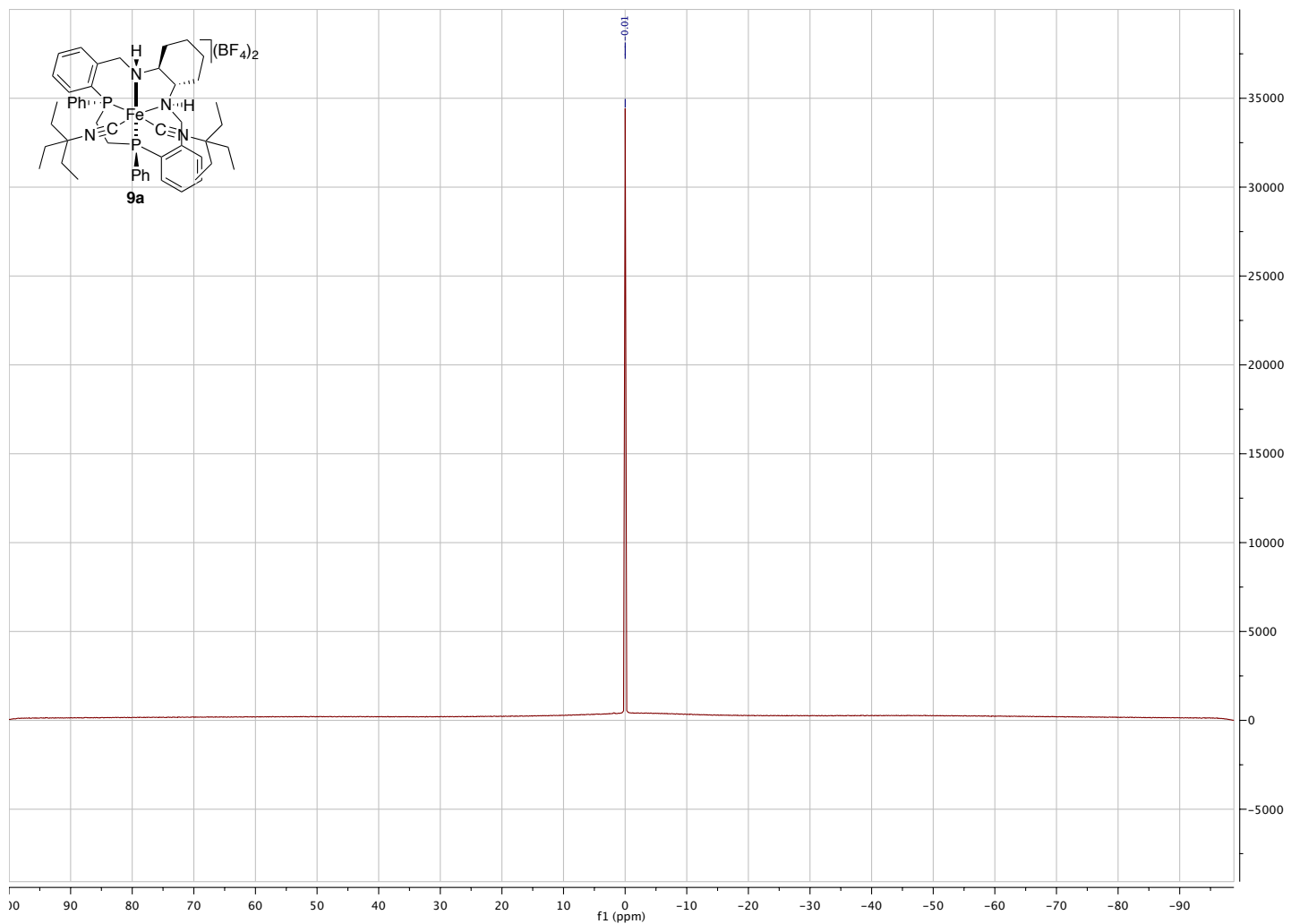




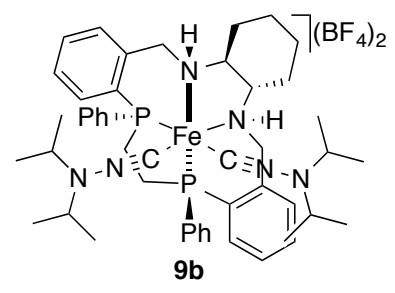

Synthesis of $\left[\mathrm{Fe}\left(\mathrm{CNN}^{i} \mathrm{Pr}_{2}\right)_{2}\left(\left(S_{\mathrm{P}}, S_{\mathrm{P}}, S_{\mathrm{C}}, S_{\mathrm{C}}\right)-7\right)\right]\left(\mathrm{BF}_{4}\right)_{2}$, 9b. The title

compound was prepared following a literature procedure. ${ }^{\mathrm{S} 4} \mathrm{~A}$ flame-dried $50 \mathrm{~mL}$ Young flask was charged with 8 (1.000 g, $1.18 \mathrm{mmol}), \mathrm{CH}_{2} \mathrm{Cl}_{2}$ (12 mL; degassed by 10 vacuum / argon cycles), and $N$-isocyano- $N$-isopropylpropan-2-amine ${ }^{\mathrm{S} 4}(0.744 \mathrm{~g}, 5.89 \mathrm{mmol}, 5.0$ equiv), and the resulting solution was stirred for $24 \mathrm{~h}$ at $50{ }^{\circ} \mathrm{C}$ (Notes: $\mathrm{N}$-Isocyano- $\mathrm{N}$-isopropylpropan-2-amine was purified by flash column chromatography (pentane : $\mathrm{Et}_{2} \mathrm{O}=19: 1$ ) directly before use; it was weighted and added using a syringe). Then, the solution was cooled to room temperature and filtered into a flamedried $100 \mathrm{~mL}$ Schlenk flask using a cannula filter. The Young flask was rinsed twice with $\mathrm{CH}_{2} \mathrm{Cl}_{2}$ ( $2 \times 5 \mathrm{~mL}$; degassed by 10 vacuum / argon cycles), and the solvent was removed using an external cooling trap. The crude product was dissolved in $\mathrm{CH}_{2} \mathrm{Cl}_{2}(10 \mathrm{~mL}$; degassed by 10 vacuum / argon cycles) and layered with hexane $(80 \mathrm{~mL})$. After 2 days, a brown oil had separated, and the supernatant was removed using a cannula. This process was repeated once to afford the product as an orange solid after drying under high vacuum. Yield: $1.169 \mathrm{~g}$ (97\%, single isomer). ${ }^{1} \mathbf{H}$ NMR $\left(300 \mathrm{MHz}, \mathrm{CD}_{2} \mathrm{Cl}_{2}\right): \delta 7.91-7.81(m, 2 \mathrm{H}, \mathrm{Ar}-H), 7.79-7.63(m, 3 \mathrm{H}, \mathrm{Ar}-H), 7.60-7.48(m, 3 \mathrm{H}$, Ar- $H), 7.47-7.39(m, 2 \mathrm{H}, \mathrm{Ar}-H), 7.39-7.30(m, 2 \mathrm{H}, \mathrm{Ar}-H), 7.25-7.16(m, 2 \mathrm{H}, \mathrm{Ar}-H), 7.06(d d d$, $\left.{ }^{3} J_{\mathrm{H}, \mathrm{H}^{\prime}}=7.8,7.7 \mathrm{~Hz},{ }^{4} J_{\mathrm{H}, \mathrm{H}^{\prime}}=2.1 \mathrm{~Hz}, 2 \mathrm{H}, \mathrm{Ar}-H\right), 6.23-6.14(m, 2 \mathrm{H}, \mathrm{Ar}-H), 5.51\left(d,{ }^{3} J_{\mathrm{H}, \mathrm{H}^{\prime}}=12.1 \mathrm{~Hz}\right.$, $1 \mathrm{H}, \mathrm{NH}), 4.71\left(d,{ }^{2} J_{\mathrm{H}, \mathrm{H}^{\prime}}=19.1 \mathrm{~Hz}, 1 \mathrm{H}, \mathrm{NCHH}\right), 4.61\left(d,{ }^{2} J_{\mathrm{H}, \mathrm{H}^{\prime}}=19.1 \mathrm{~Hz}, 1 \mathrm{H}, \mathrm{NCHH}\right), 4.17-4.04$ $(m, 2 \mathrm{H}, \mathrm{NCHH}), 3.64-3.39(m, 1 \mathrm{H}, \mathrm{PC} H \mathrm{H}), 3.30-3.14\left(m, 4 \mathrm{H}, \mathrm{NCH}\left(\mathrm{CH}_{3}\right)_{2}\right), 3.14-2.78(m, 3 \mathrm{H}$, $\mathrm{PC} H \mathrm{H}), 2.55-2.44(m, 1 \mathrm{H}, \mathrm{CHH}), 2.39-2.16(m, 2 \mathrm{H}, \mathrm{NCH}+\mathrm{CHH}), 1.89-1.73(m, 2 \mathrm{H}, \mathrm{NCH}+$ $\mathrm{CHH}), 1.68-1.57(m, 1 \mathrm{H}, \mathrm{CHH}), 1.50-1.22(m, 2 \mathrm{H}, \mathrm{NH}+\mathrm{CHH}), 1.18-1.08(m, 1 \mathrm{H}, \mathrm{CHH}), 1.05$ $\left(d,{ }^{3} \mathrm{~J}_{\mathrm{H}, \mathrm{H}^{\prime}}=6.6 \mathrm{~Hz}, 6 \mathrm{H}, \mathrm{NCH}\left(\mathrm{CH}_{3}\right)\left(\mathrm{CH}_{3}\right)\right), 1.02\left(d,{ }^{3} \mathrm{~J}_{\mathrm{H}, \mathrm{H}^{\prime}}=6.7 \mathrm{~Hz}, 6 \mathrm{H}, \mathrm{NCH}\left(\mathrm{CH}_{3}\right)\left(\mathrm{CH}_{3}\right)\right), 0.93(d$, 
$\left.{ }^{3} J_{\mathrm{H}, \mathrm{H}^{\prime}}=6.5 \mathrm{~Hz}, 6 \mathrm{H}, \mathrm{NCH}\left(\mathrm{CH}_{3}\right)\left(\mathrm{CH}_{3}\right)\right), 0.83\left(d,{ }^{3} J_{\mathrm{H}, \mathrm{H}^{\prime}}=6.4 \mathrm{~Hz}, 6 \mathrm{H}, \mathrm{NCH}\left(\mathrm{CH}_{3}\right)\left(\mathrm{CH}_{3}\right)\right), 0.81-0.68$ $(m, 1 \mathrm{H}, \mathrm{CHH}),-0.28\left(q d,{ }^{3} \mathrm{~J}_{\mathrm{H}, \mathrm{H}^{\prime}}=12.6,3.5 \mathrm{~Hz}, 1 \mathrm{H}, \mathrm{CHH}\right) .{ }^{31} \mathbf{P}\left\{{ }^{1} \mathbf{H}\right\} \mathbf{N M R}\left(122 \mathrm{MHz}, \mathrm{CD}_{2} \mathrm{Cl}_{2}\right)$ : $\left.\delta 91.8\left(d,{ }^{2} J_{\mathrm{P}, \mathrm{P}},=20.0 \mathrm{~Hz}\right), 90.2\left(d,{ }^{2} J_{\mathrm{P}, \mathrm{P}},=20.0 \mathrm{~Hz}\right) .{ }^{13} \mathbf{C}^{31}{ }^{31} \mathbf{P},{ }^{1} \mathbf{H}\right\} \mathbf{N M R}\left(126 \mathrm{MHz}, \mathrm{CD}_{2} \mathrm{Cl}_{2}\right)$ : $\delta 142.9$ (arom.), 141.9 (arom.), $138.4\left(\mathrm{CNN}^{i} \mathrm{Pr}_{2}\right), 135.7$ ( $\left.C \mathrm{NN}^{i} \mathrm{Pr}_{2}\right), 135.5$ (arom.), 134.1 (arom.), 133.9 (arom.), 133.7 (arom.), 132.8 (arom.), 132.4 (2C, arom.), 132.33 (arom.), 132.26 (arom.), 132.2 (arom.), 131.81 (arom.), 131.76 (arom.), 131.6 (arom.), 130.5 (arom.), 130.3 (arom.), 130.0 (arom.), 126.0 (arom.), 124.1 (arom.), $67.7(\mathrm{NCH}), 64.5(\mathrm{NCH}), 56.2\left(\mathrm{NCH}_{2}\right), 54.8\left(\mathrm{NCH}\left(\mathrm{CH}_{3}\right)_{2}\right)$, $54.3\left(\mathrm{NCH}\left(\mathrm{CH}_{3}\right)_{2}\right), 51.0\left(\mathrm{NCH}_{2}\right), 32.2\left(\mathrm{PCH}_{2}\right), 31.4\left(\mathrm{CH}_{2}\right), 29.9\left(\mathrm{CH}_{2}\right), 27.6\left(\mathrm{PCH}_{2}\right), 24.8\left(\mathrm{CH}_{2}\right)$, $24.7\left(\mathrm{CH}_{2}\right), 21.0\left(\mathrm{NCH}\left(\mathrm{CH}_{3}\right)\left(\mathrm{CH}_{3}\right)\right), 20.4\left(\mathrm{NCH}\left(\mathrm{CH}_{3}\right)\left(\mathrm{CH}_{3}\right)\right), 20.1 \quad\left(\mathrm{NCH}\left(\mathrm{CH}_{3}\right)\left(\mathrm{CH}_{3}\right)\right), \quad 19.6$ $\left(\mathrm{NCH}\left(\mathrm{CH}_{3}\right)\left(\mathrm{CH}_{3}\right)\right) .{ }^{11} \mathbf{B}$ NMR $\left(96 \mathrm{MHz}, \mathrm{CD}_{2} \mathrm{Cl}_{2}\right): \delta 0.0(s)$. IR (liquid film, $\left.\mathrm{cm}^{-1}\right): 3245(\mathrm{~N}-\mathrm{H})$, 3224 (N-H), 3059 (C-H), 2979 (C-H), 2937 (C-H), 2870 (C-H), 2110 (isonitrile), 1630, 1592, 1481, $1463,1450,1435,1389,1372,1325,1282,1265,1243,1187,1166,1122,1050,1035$. HRMS (MALDI): Calcd. for $\mathrm{C}_{48} \mathrm{H}_{66} \mathrm{BF}_{4} \mathrm{FeN}_{6} \mathrm{P}_{2} \mathrm{~m} / z$ 931.4206, found $m / z 931.4193$ [M-BF 4$]^{+}$. EA: Calcd. for $\mathrm{C}_{48} \mathrm{H}_{66} \mathrm{~B}_{2} \mathrm{~F}_{8} \mathrm{FeN}_{6} \mathrm{P}_{2}: \mathrm{C}, 56.61 ; \mathrm{H}, 6.53 ; \mathrm{N}, 8.25$; found: $\mathrm{C}, 56.38 ; \mathrm{H}, 6.74 ; \mathrm{N}, 8.28$. 
Figure S48: ${ }^{1} \mathrm{H}$ NMR spectrum of $9 \mathbf{b}\left(300 \mathrm{MHz}, \mathrm{CD}_{2} \mathrm{Cl}_{2}\right)$.

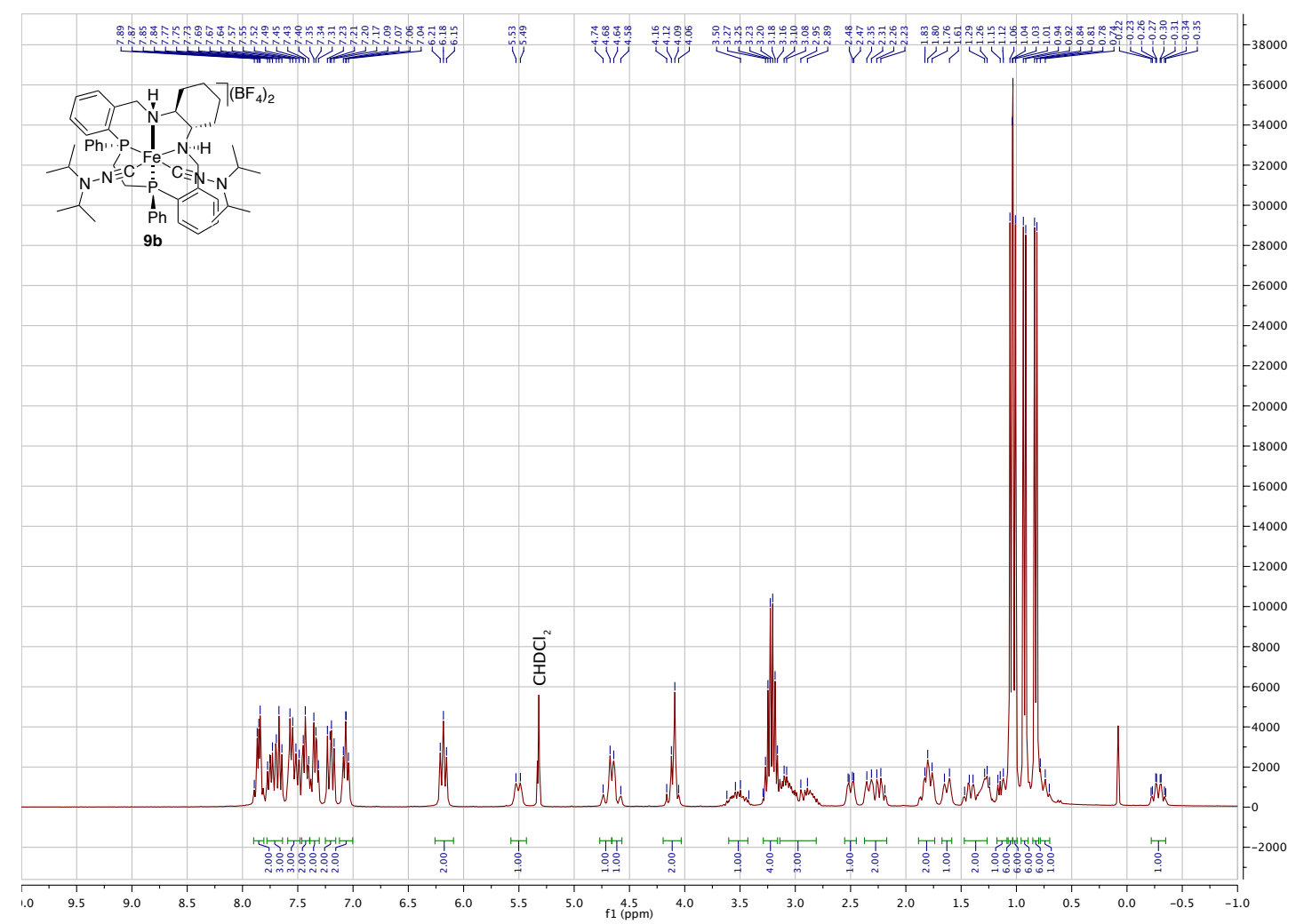

Figure S49: ${ }^{31} \mathrm{P}\left\{{ }^{1} \mathrm{H}\right\}$ NMR spectrum of $9 b\left(122 \mathrm{MHz}, \mathrm{CD}_{2} \mathrm{Cl}_{2}\right)$.

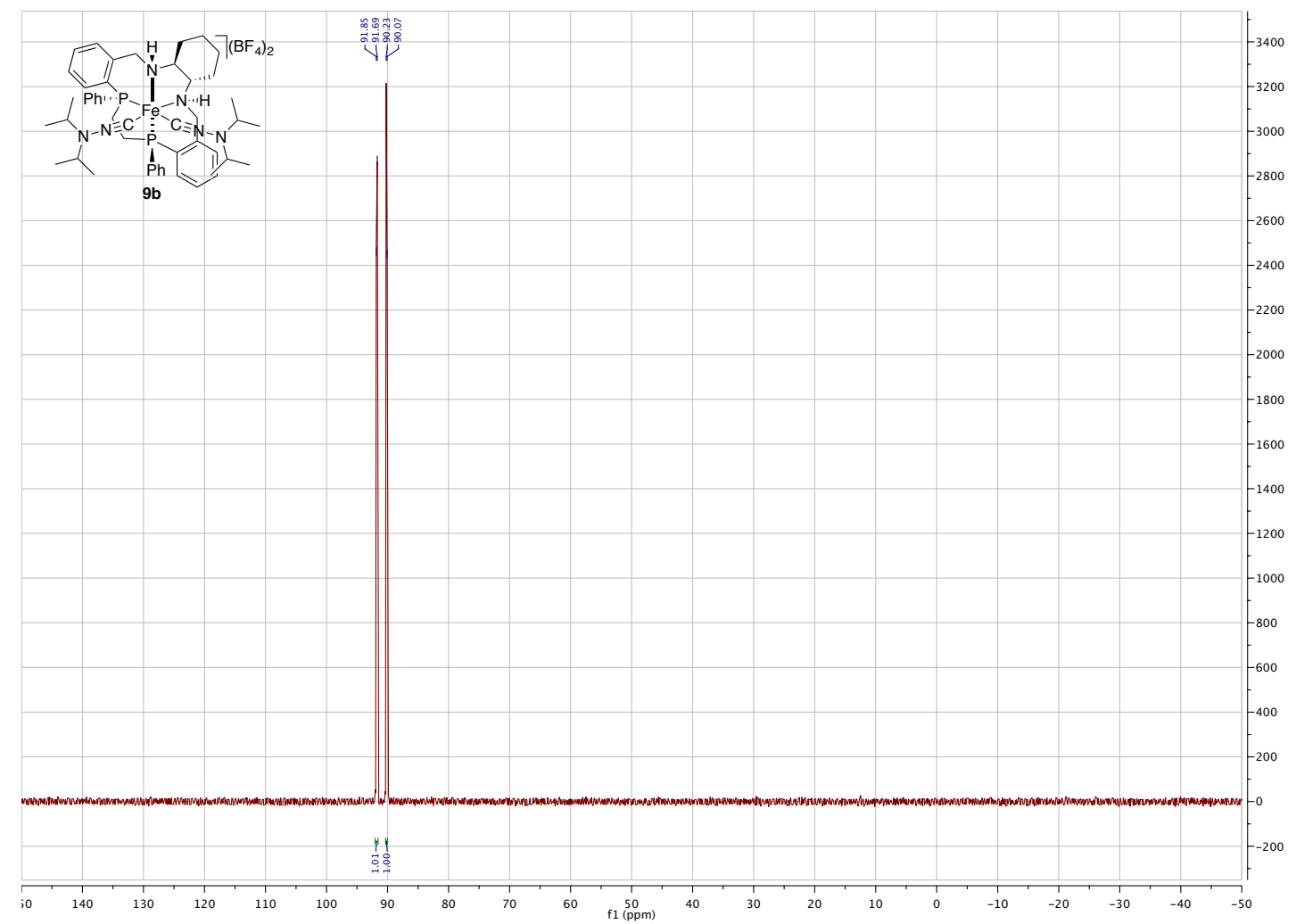


Figure S50: ${ }^{13} \mathrm{C}\left\{{ }^{31} \mathrm{P},{ }^{1} \mathrm{H}\right\} \mathrm{NMR}$ spectrum of $9 \mathbf{b}\left(126 \mathrm{MHz}, \mathrm{CD}_{2} \mathrm{Cl}_{2}\right)$.

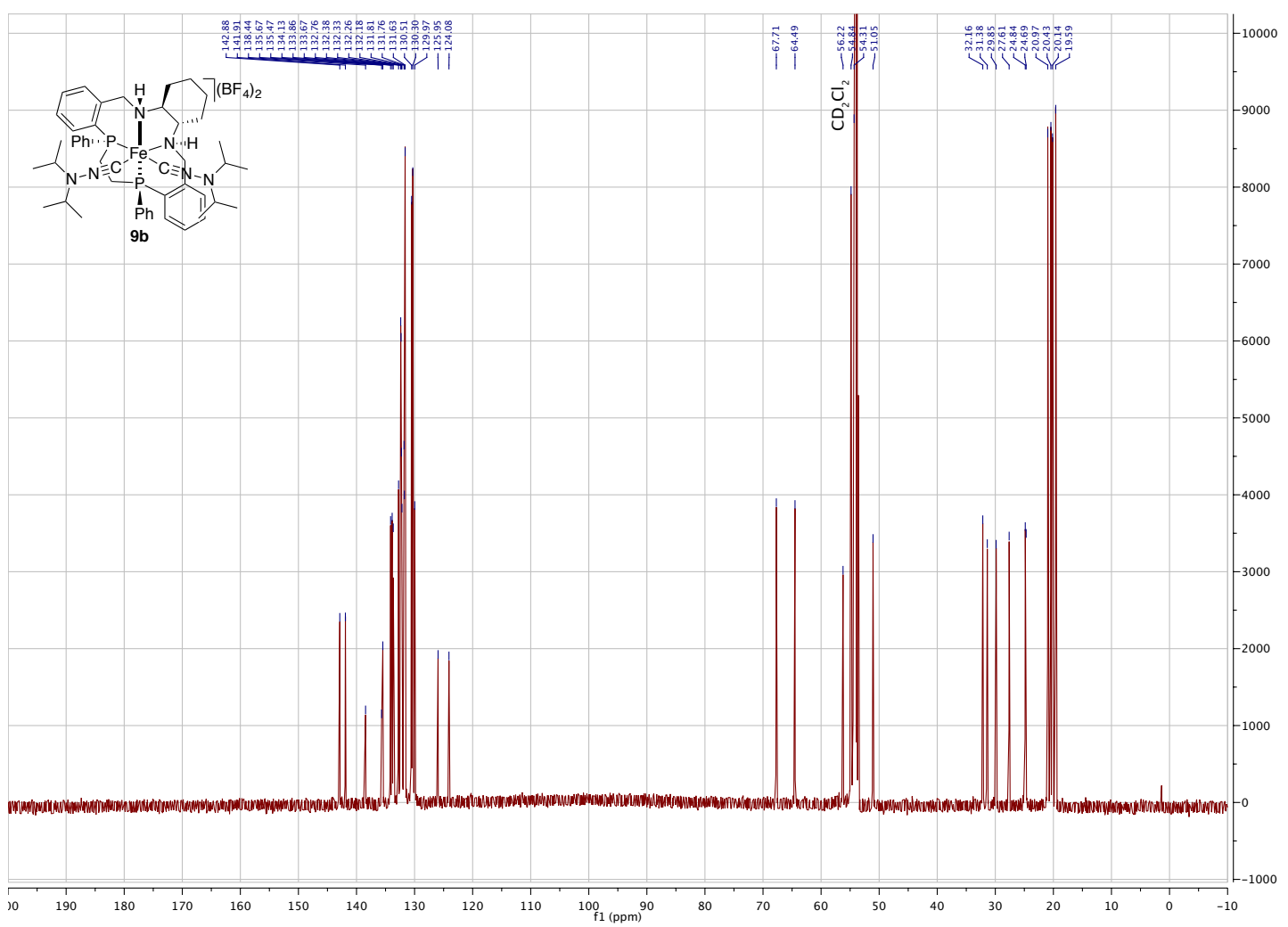

Figure S51: ${ }^{11} \mathrm{~B}$ NMR spectrum of $9 \mathbf{b}\left(96 \mathrm{MHz}, \mathrm{CD}_{2} \mathrm{Cl}_{2}\right)$.

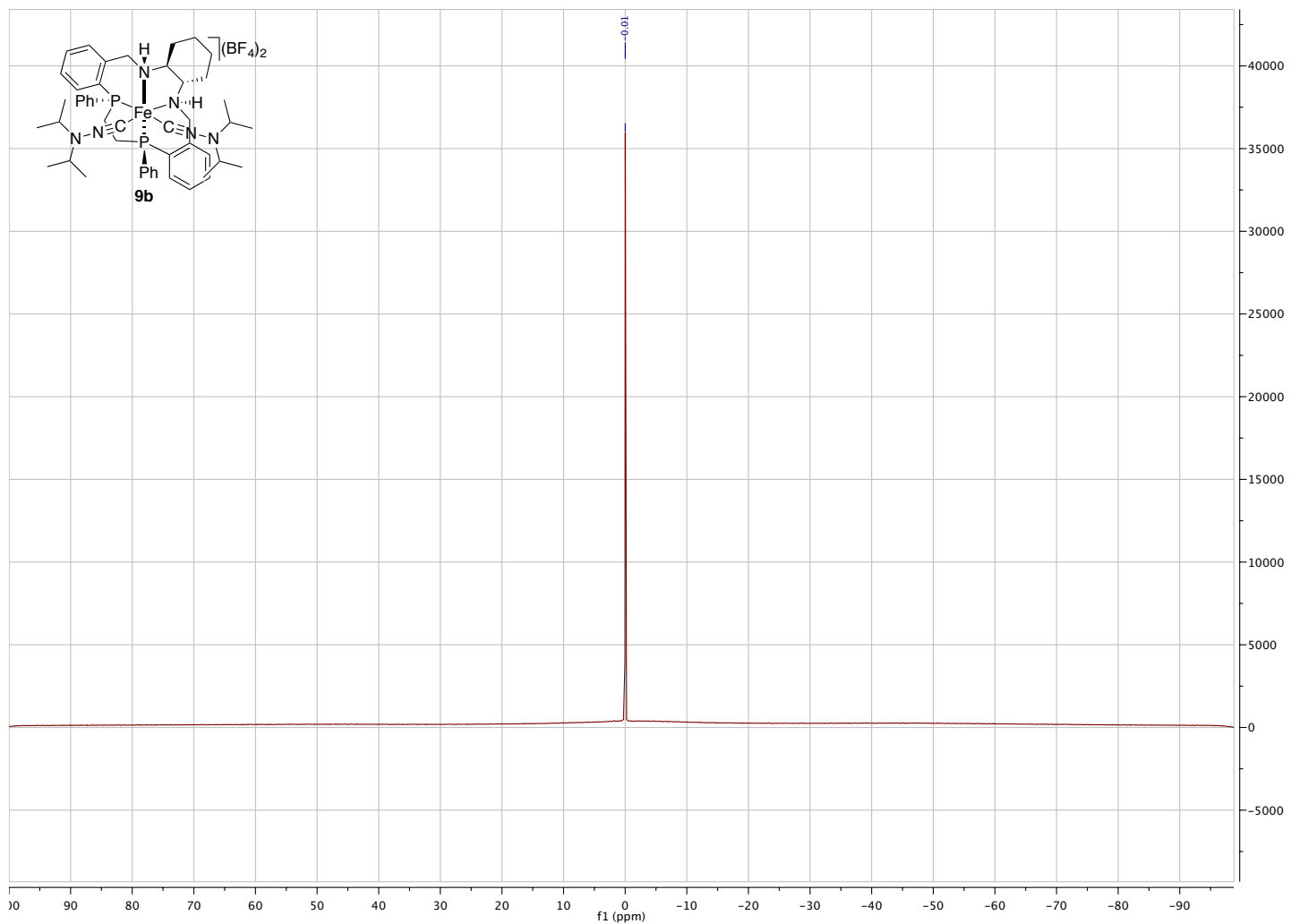




\section{Asymmetric Transfer Hydrogenation of Ketones 10a-10i and Imine 10j}

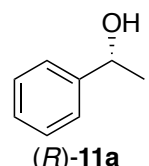

Synthesis of (+)-(R)-1-Phenylethan-1-ol, 11a. In a glove box, a vial was charged with complex 9a $(10.2 \mathrm{mg}, 10.0 \mu \mathrm{mol}, 0.01 \mathrm{~mol} \%)$ or $9 \mathbf{b}(20.4 \mathrm{mg}, 20.0 \mu \mathrm{mol}, 0.02 \mathrm{~mol} \%)$ and sodium tert-butoxide $(24.0 \mathrm{mg}, 250 \mu \mathrm{mol}, 0.25 \mathrm{~mol} \%$ (for 9a) or $38.4 \mathrm{mg}, 400 \mu \mathrm{mol}, 0.4 \mathrm{~mol} \%$ (for 9b)). Then, the vial was closed with a plastic cap and removed from the glove box. The solids were added to a flame-dried $1000 \mathrm{~mL}$ two-neck round-bottom flask with reflux condenser using a paper funnel and dried for 5 min under high vacuum. 2-Propanol $(250 \mathrm{~mL})$ was added using a Teflon cannula, and the flask was immersed in a preheated oil bath at $60{ }^{\circ} \mathrm{C}$ (for 9a) or $75{ }^{\circ} \mathrm{C}$ (for $9 \mathbf{b}$ ). After $5 \mathrm{~min}$, 2-propanol (250 mL) was added using a Teflon cannula. After additional 5 min, acetophenone (10a) $(11.7 \mathrm{~mL}, 100 \mathrm{mmol})$ was added, and the solution was stirred at the specified temperature for $2 \mathrm{~h}$ (for $\mathbf{9 a}$ ) or $3 \mathrm{~h}$ (for $\mathbf{9 b}$ ), when the reaction mixtures showed conversions close to equilibrium. The solvent was removed carefully at the rotary evaporator $\left(40{ }^{\circ} \mathrm{C}, 105 \mathrm{mbar}\right)$, and the crude product was purified by flash column chromatography on silica gel $\left(\mathrm{Et}_{2} \mathrm{O}:\right.$ pentane $=1: 9$ to $3: 2)$ to afford the title product as a clear liquid. Yield with catalyst 9a: $11.22 \mathrm{~g}(91.8 \%, 96.3 \% e e)$. Yield with catalyst 9b: $11.08 \mathrm{~g}(90.7 \%, 97.8 \%$ ee $) .{ }^{1} \mathbf{H}$ NMR $\left(300 \mathrm{MHz}, \mathrm{CDCl}_{3}\right): \delta 7.45-7.27(\mathrm{~m}$, $5 \mathrm{H}, \mathrm{Ar}-\mathrm{H}), 4.92\left(d,{ }^{3} J_{\mathrm{H}, \mathrm{H}^{\prime}}=6.4 \mathrm{~Hz}, 1 \mathrm{H}, \mathrm{CHOH}\right), 2.10(\mathrm{br} s, 1 \mathrm{H}, \mathrm{OH}), 1.53\left(d,{ }^{3} J_{\mathrm{H}, \mathrm{H}^{\prime}}=6.4 \mathrm{~Hz}, 1 \mathrm{H}\right.$, $\left.\mathrm{CH}_{3}\right) .{ }^{13} \mathbf{C}\left\{{ }^{1} \mathbf{H}\right\} \mathbf{N M R}\left(75 \mathrm{MHz}, \mathrm{CDCl}_{3}\right.$ ): $\delta 145.9$ (arom.), 128.6 (arom.), 127.5 (arom.), 125.5 (arom.), $70.5(\mathrm{CHOH}), 25.3\left(\mathrm{CH}_{3}\right)$. IR (liquid film, $\left.\mathrm{cm}^{-1}\right): 3338(\mathrm{O}-\mathrm{H}), 3085(\mathrm{C}-\mathrm{H}), 3062(\mathrm{C}-\mathrm{H})$, 3029 (C-H), 2972 (C-H), 2926 (C-H), 2872 (C-H), 1602, 1493, 1450, 1407, 1368, 1327, 1283, 1261, 1203, 1177, 1156, 1098, 1075, 1028, 1009. HRMS (EI): Calcd. for $\mathrm{C}_{8} \mathrm{H}_{10} \mathrm{O} m / z=122.0727$, found $m / z=122.0727[\mathrm{M}]^{+} .[\boldsymbol{\alpha}]_{\mathbf{D}}{ }^{20}:+61.3\left(\mathrm{c}=1.0, \mathrm{CHCl}_{3}, 96.3 \%\right.$ ee, product from reaction with 
catalyst $9 \mathbf{a}),+61.7\left(\mathrm{c}=1.0, \mathrm{CHCl}_{3}, 97.8 \%\right.$ ee, product from reaction with catalyst $\left.\mathbf{9 b}\right) . \mathbf{R}_{\mathbf{f}}\left(\mathrm{Et}_{2} \mathrm{O}\right.$ : pentane $=1: 3)=0.33$. GC: $\beta$-DEX column, $100{ }^{\circ} \mathrm{C}$ isotherm, retention times $t_{\mathrm{R}}$ (major) $=$ $22.2 \mathrm{~min}, t_{\mathrm{R}}($ minor $)=24.1 \mathrm{~min}$. Analytical data are in agreement with literature data. ${ }^{\mathrm{S}, 5}$ The absolute configuration was determined to be $(R)$ by comparison with literature data. ${ }^{\mathrm{S} 5}$

Figure S52: GC trace of enantioenriched $(R)-11 a$ (obtained with catalyst 9a).

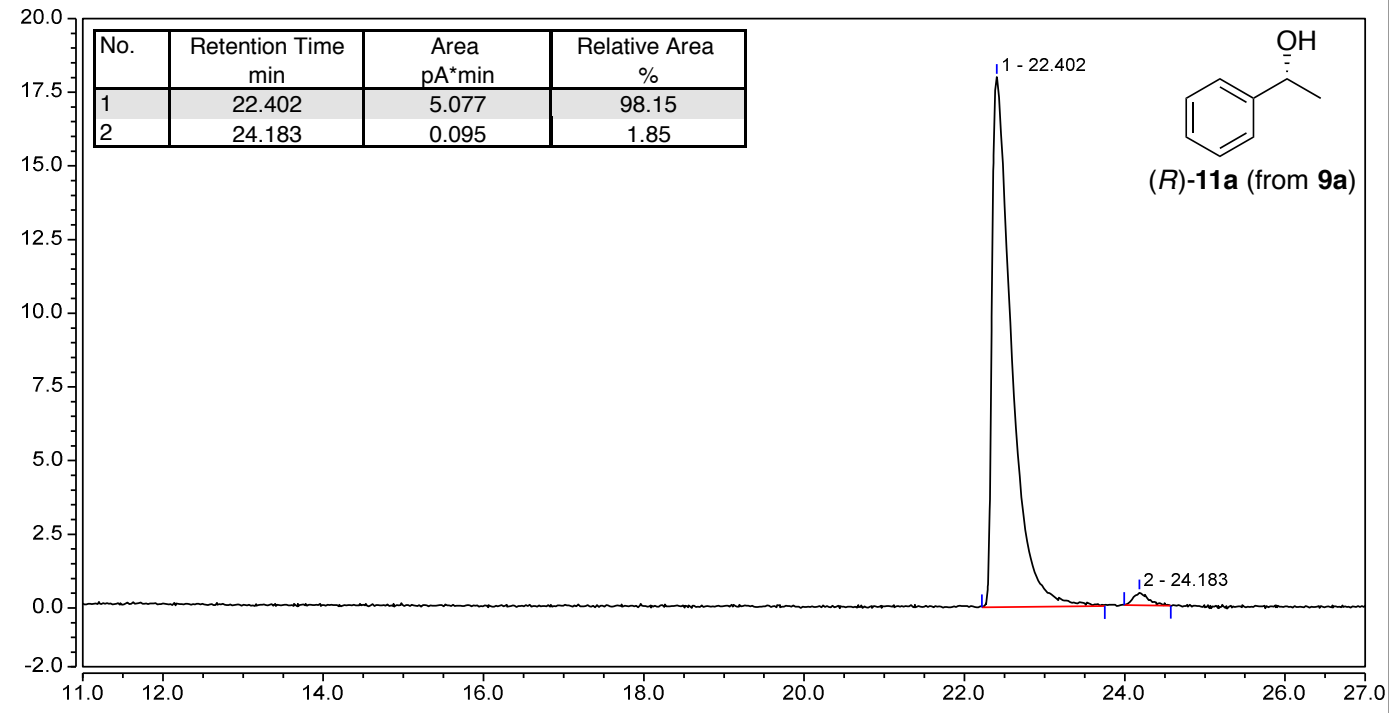

Figure S53: GC trace of enantioenriched $(R)-11 a$ (obtained with catalyst 9b).

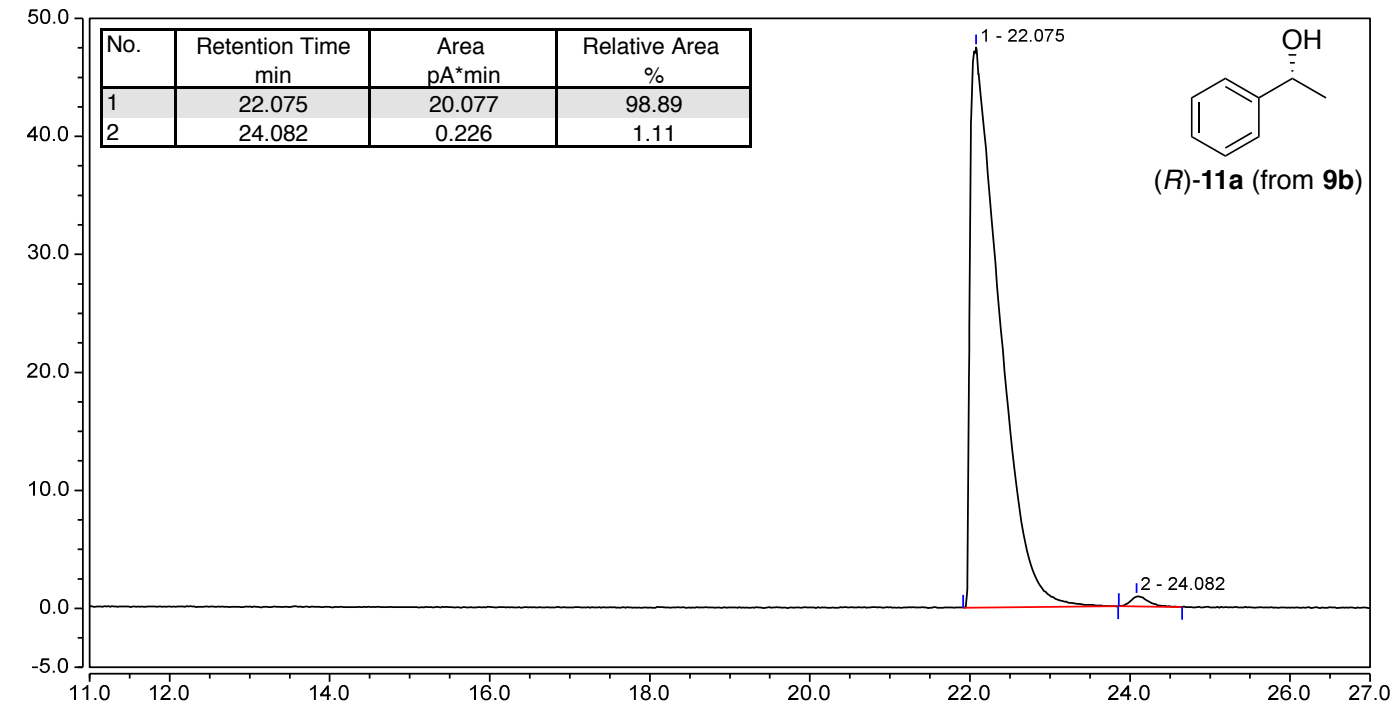


Figure S54: ${ }^{1} \mathrm{H}$ NMR spectrum of enantioenriched $(R)-\mathbf{1 1 a}\left(300 \mathrm{MHz}, \mathrm{CDCl}_{3}\right)$.

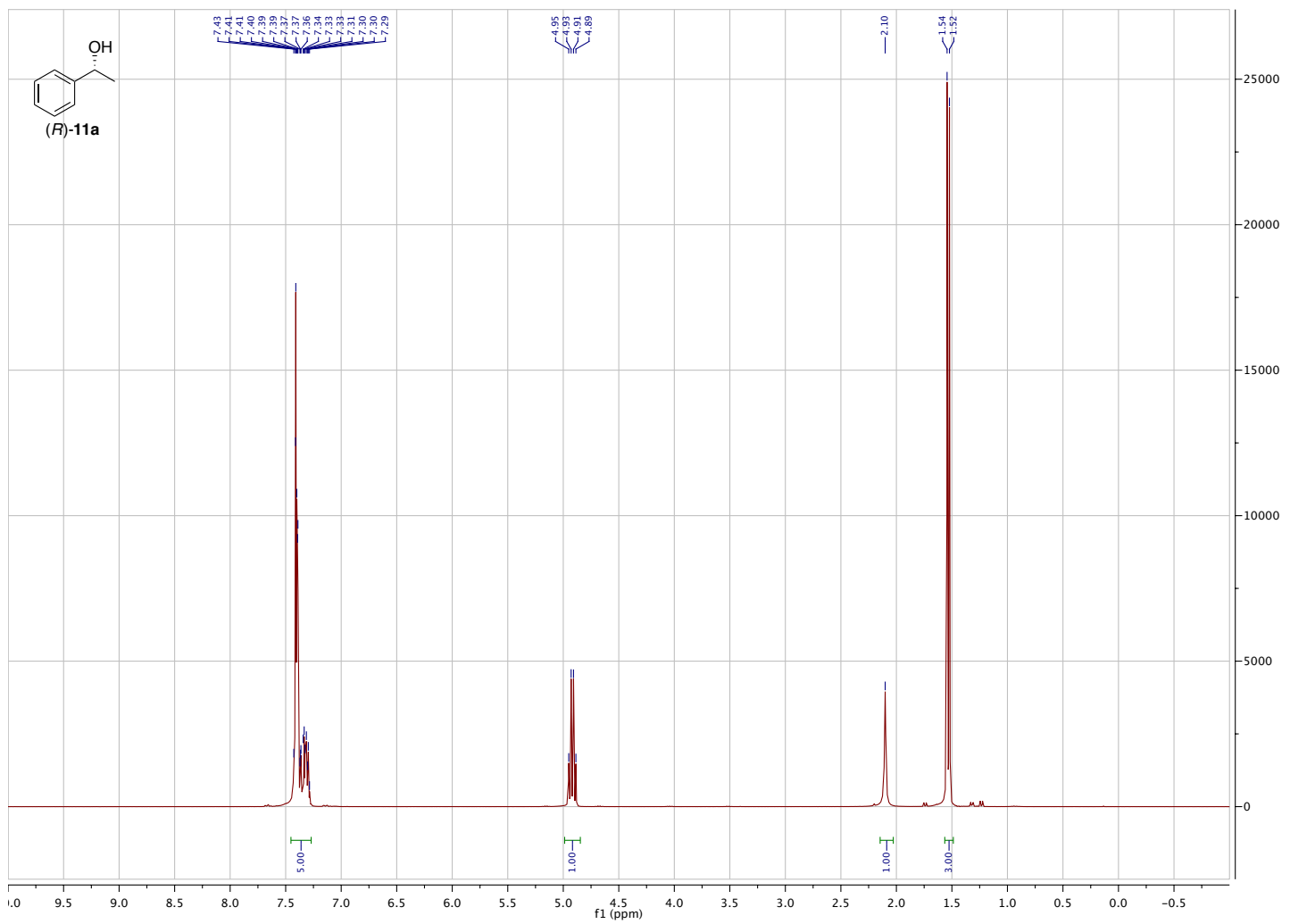

Figure S55: ${ }^{13} \mathrm{C}\left\{{ }^{1} \mathrm{H}\right\}$ NMR spectrum of enantioenriched $(R)-11 a\left(75 \mathrm{MHz}, \mathrm{CDCl}_{3}\right)$.

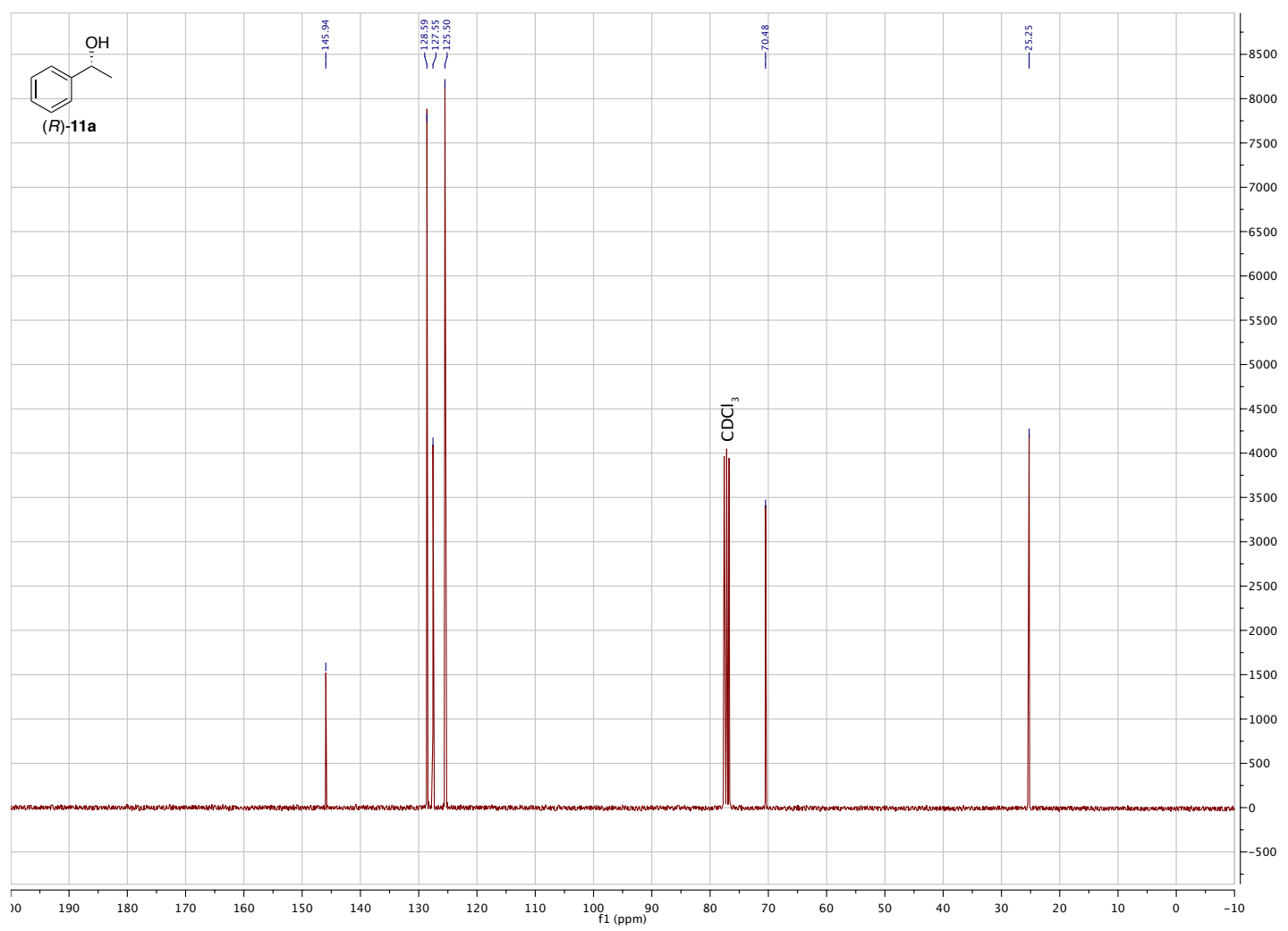




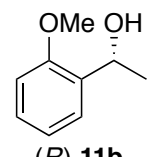

Synthesis of (+)-(R)-1-(2-Methoxyphenyl)ethan-1-ol, 11b. In a glove box, a vial was charged with complex 9a (40.7 mg, $40.0 \mu \mathrm{mol}, 0.04 \mathrm{~mol} \%)$ and sodium tert-butoxide $(57.7 \mathrm{mg}$, $600 \mu \mathrm{mol}, 0.60 \mathrm{~mol} \%$ ). The vial was closed with a plastic cap and removed from the glove box. The solids were added to a flame-dried $1000 \mathrm{~mL}$ two-neck round-bottom flask using a paper funnel and dried for 5 min under high vacuum. 2-Propanol $(250 \mathrm{~mL})$ was added using a Teflon cannula, and the flask was immersed in a preheated oil bath at $60{ }^{\circ} \mathrm{C}$. After $5 \mathrm{~min}, 2$-propanol $(250 \mathrm{~mL})$ was added using a Teflon cannula. After additional $5 \mathrm{~min}, 2^{2}$-methoxyacetophenone (10b) (13.7 mL, $100 \mathrm{mmol}$ ) was added, and the solution was stirred at $60{ }^{\circ} \mathrm{C}$ for $2.5 \mathrm{~h}$, when the reaction mixture showed almost full conversion. The solvent was removed at the rotary evaporator $\left(40^{\circ} \mathrm{C}, 90 \mathrm{mbar}\right)$, and the crude product was purified by flash column chromatography on silica gel $\left(\mathrm{Et}_{2} \mathrm{O}:\right.$ pentane $=$ $1: 9$ to $3: 2)$ to afford the title product as a slightly yellowish liquid. Yield: $15.04 \mathrm{~g}(98.8 \%$, 99.4\% eе). ${ }^{1} \mathbf{H}$ NMR (300 MHz, $\left.\mathrm{CDCl}_{3}\right): \delta 7.35\left(d d,{ }^{3} J_{\mathrm{H}, \mathrm{H}},=7.5 \mathrm{~Hz},{ }^{4} J_{\mathrm{H}, \mathrm{H}}=1.7 \mathrm{~Hz}, 1 \mathrm{H}, \mathrm{Ar}-H\right)$, $7.25\left(d d d,{ }^{3} J_{\mathrm{H}, \mathrm{H}},=8.3,7.5 \mathrm{~Hz},{ }^{4} J_{\mathrm{H}, \mathrm{H}}=1.7 \mathrm{~Hz}, 1 \mathrm{H}, \mathrm{Ar}-H\right), 6.97\left(d d d,{ }^{3} J_{\mathrm{H}, \mathrm{H}}=7.5,7.5 \mathrm{~Hz},{ }^{4} J_{\mathrm{H}, \mathrm{H}},=\right.$ $1.1 \mathrm{~Hz}, 1 \mathrm{H}, \operatorname{Ar}-H), 6.89\left(\mathrm{br} d,{ }^{3} J_{\mathrm{H}, \mathrm{H}^{\prime}}=8.3 \mathrm{~Hz}, 1 \mathrm{H}, \operatorname{Ar}-H\right), 5.10\left(q,{ }^{3} J_{\mathrm{H}, \mathrm{H}^{\prime}}=6.5 \mathrm{~Hz}, 1 \mathrm{H}, \mathrm{CHOH}\right), 3.86$ $\left(s, 3 \mathrm{H}, \mathrm{OCH}_{3}\right), 2.69($ br $s, 1 \mathrm{H}, \mathrm{OH}), 1.51\left(d,{ }^{3} J_{\mathrm{H}, \mathrm{H}^{\prime}}=6.5 \mathrm{~Hz}, 3 \mathrm{H}, \mathrm{CHCH}_{3}\right) .{ }^{13} \mathbf{C}\left\{{ }^{1} \mathbf{H}\right\} \mathbf{~ N M R}(75 \mathrm{MHz}$, $\mathrm{CDCl}_{3}$ ): $\delta 156.6$ (arom.), 133.6 (arom.), 128.4 (arom.), 126.2 (arom.), 120.9 (arom.), 110.5 (arom.), $66.6(\mathrm{CHOH}), 55.4\left(\mathrm{OCH}_{3}\right), 23.0\left(\mathrm{CHCH}_{3}\right)$. IR (liquid film, $\left.\mathrm{cm}^{-1}\right): 3363(\mathrm{O}-\mathrm{H}), 3067(\mathrm{C}-\mathrm{H}), 3034$ (C-H), 2969 (C-H), 2928 (C-H), 2836 (C-H), 1601, 1587, 1490, 1464, 1455, 1438, 1403, 1364, 1282, 1235, 1193, 1174, 1161, 1124, 1075, 1050, 1028, 1009. HRMS (EI): Calcd. for $\mathrm{C}_{9} \mathrm{H}_{12} \mathrm{O}_{2}$ $m / z=152.0832$, found $m / z=152.0831[\mathrm{M}]^{+} \cdot[\boldsymbol{\alpha}]_{\mathbf{D}}{ }^{20}:+28.0\left(\mathrm{c}=1.0, \mathrm{CHCl}_{3}\right) . \mathbf{R}_{\mathbf{f}}\left(\mathrm{Et}_{2} \mathrm{O}:\right.$ pentane $=$ $1: 3)=0.27$. GC: $\beta$-DEX column, $130{ }^{\circ} \mathrm{C}$ isotherm, retention times $t_{\mathrm{R}}$ (minor) $=20.2 \mathrm{~min}$, $t_{\mathrm{R}}($ major $)=20.9 \mathrm{~min}$. Analytical data are in agreement with literature data. ${ }^{\mathrm{S}, 5}$ 
Figure S56: ${ }^{1} \mathrm{H}$ NMR spectrum of enantioenriched $(R)-\mathbf{1 1 b}\left(300 \mathrm{MHz}, \mathrm{CDCl}_{3}\right)$.

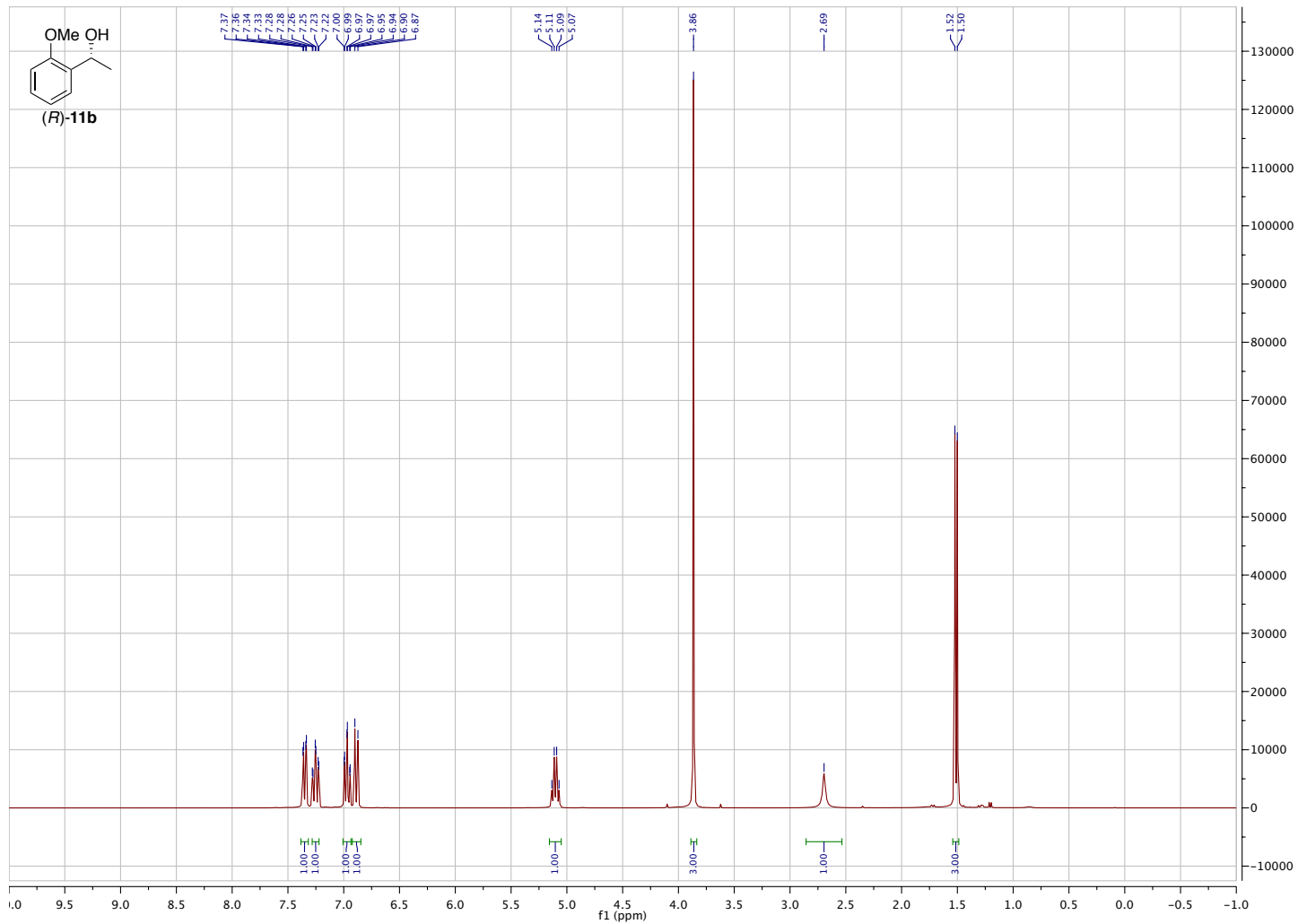

Figure S57: ${ }^{13} \mathrm{C}\left\{{ }^{1} \mathrm{H}\right\}$ NMR spectrum of enantioenriched $(R)-\mathbf{1 1 b}\left(75 \mathrm{MHz}, \mathrm{CDCl}_{3}\right)$.

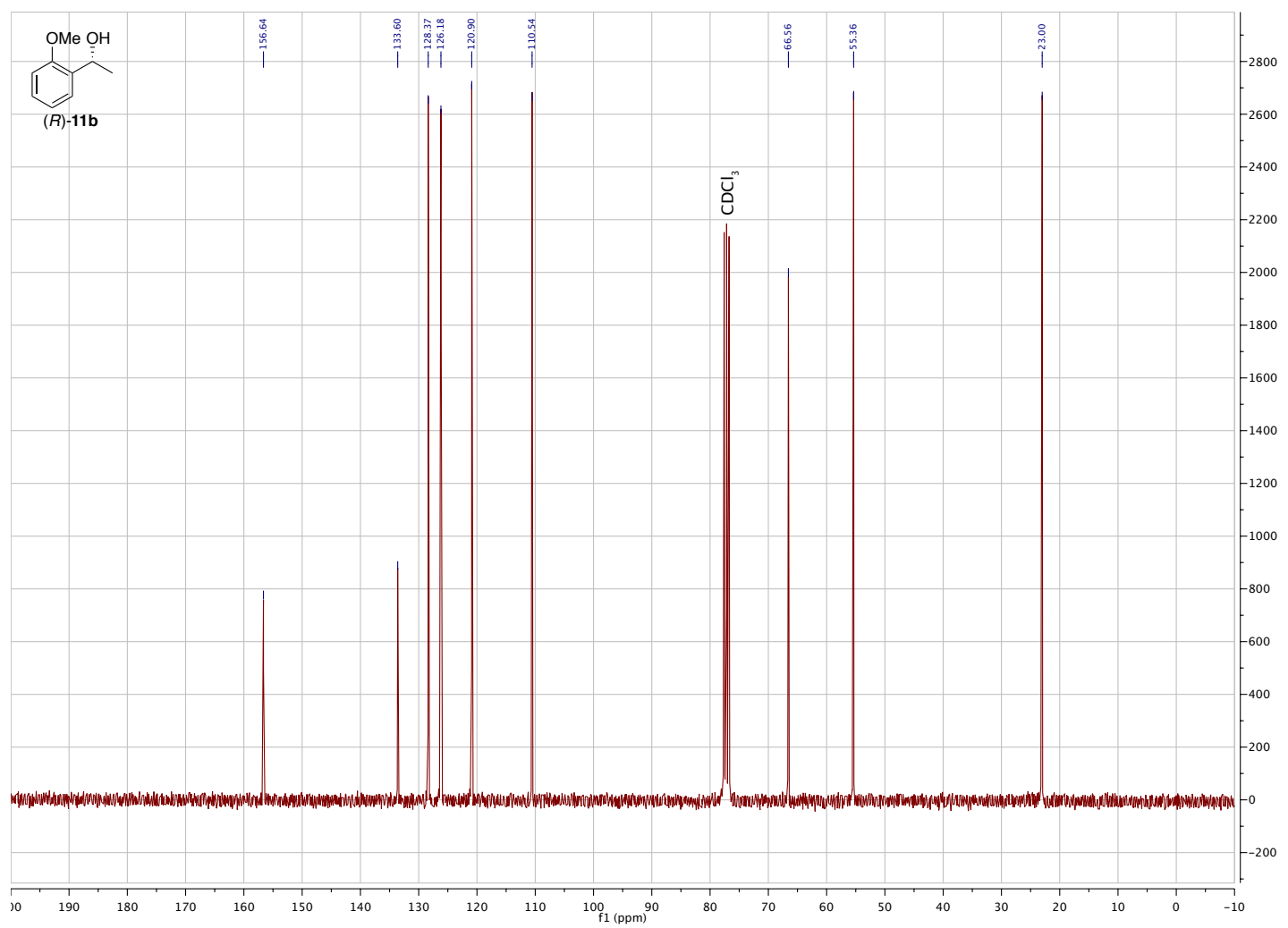


Figure S58: GC trace of enantioenriched $(R)-\mathbf{1 1 b}$ (obtained with catalyst 9a).
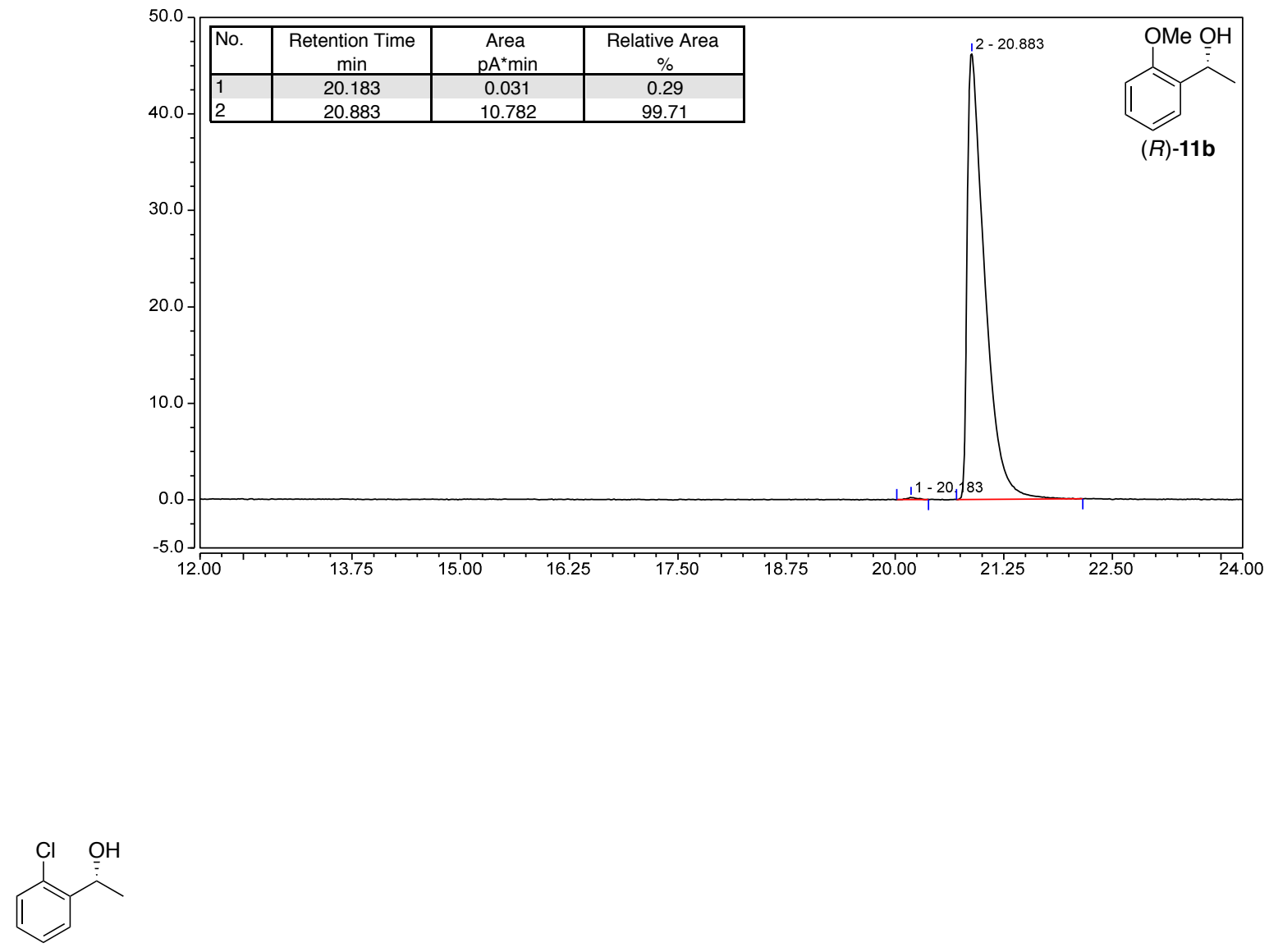

(R)-11c Synthesis of $(+)-(R)-1-(2-C h l o r o p h e n y l) e t h a n-1-o l, 11 c$. In a glove box, a vial was charged with complex 9b $(40.7 \mathrm{mg}, 40.0 \mu \mathrm{mol}, 0.04 \mathrm{~mol} \%)$ and sodium tert-butoxide $(57.7 \mathrm{mg}$, $600 \mu \mathrm{mol}, 0.60 \mathrm{~mol} \%)$. The vial was closed with a plastic cap and removed from the glove box. The solids were added to a flame-dried $1000 \mathrm{~mL}$ two-neck round-bottom flask with reflux condenser using a paper funnel and dried for $5 \mathrm{~min}$ under high vacuum. 2-Propanol $(250 \mathrm{~mL})$ was added using a Teflon cannula, and the flask was immersed in a preheated oil bath at $75{ }^{\circ} \mathrm{C}$. After $5 \mathrm{~min}$, 2-propanol $(250 \mathrm{~mL})$ was added using a Teflon cannula. After additional $5 \mathrm{~min}, 2$ '-chloroacetophenone $(\mathbf{1 0 c})(13.0 \mathrm{~mL}, 100 \mathrm{mmol})$ was added, and the solution was stirred at $75^{\circ} \mathrm{C}$ for $2.0 \mathrm{~h}$, when the reaction mixture showed almost full conversion. The solvent was removed at the rotary evaporator $\left(40{ }^{\circ} \mathrm{C}, 90 \mathrm{mbar}\right)$, and the crude product was purified by flash column chromatography on silica gel $\left(\mathrm{Et}_{2} \mathrm{O}:\right.$ pentane $=1: 9$ to $\left.3: 2\right)$ to afford the title product as a slightly yellowish liquid. 
Yield: $15.55 \mathrm{~g}\left(99.3 \%, 97.6 \%\right.$ ee). ${ }^{1} \mathbf{H}$ NMR $\left(300 \mathrm{MHz}, \mathrm{CDCl}_{3}\right): \delta 7.59\left(d d,{ }^{3} \mathrm{~J}_{\mathrm{H}, \mathrm{H}^{\prime}}=7.6 \mathrm{~Hz},{ }^{4} J_{\mathrm{H}, \mathrm{H}^{\prime}}=\right.$ $1.8 \mathrm{~Hz}, 1 \mathrm{H}, \mathrm{Ar}-H), 7.36-7.26(m, 2 \mathrm{H}, \mathrm{Ar}-H), 7.20\left(d d d,{ }^{3} J_{\mathrm{H}, \mathrm{H}^{\prime}}=7.6,7.6 \mathrm{~Hz},{ }^{4} J_{\mathrm{H}, \mathrm{H}^{\prime}}=1.8 \mathrm{~Hz}, 1 \mathrm{H}\right.$, $\operatorname{Ar}-H), 5.29\left(q,{ }^{3} J_{\mathrm{H}, \mathrm{H}^{\prime}}=6.4 \mathrm{~Hz}, 1 \mathrm{H}, \mathrm{CHOH}\right), 2.35(\mathrm{br} s, 1 \mathrm{H}, \mathrm{OH}), 1.49\left(d,{ }^{3} J_{\mathrm{H}, \mathrm{H}^{\prime}}=6.4 \mathrm{~Hz}, 3 \mathrm{H}, \mathrm{CH}_{3}\right)$. ${ }^{13} \mathbf{C}\left\{{ }^{1} \mathbf{H}\right\}$ NMR (75 MHz, $\mathrm{CDCl}_{3}$ ): $\delta 143.2$ (arom.), 131.7 (arom.), 129.5 (arom.), 128.5 (arom.), 127.3 (arom.), 126.5 (arom.), $67.0(\mathrm{CHOH}), 23.6\left(\mathrm{CH}_{3}\right)$. IR (liquid film, $\left.\mathrm{cm}^{-1}\right)$ : $3333(\mathrm{O}-\mathrm{H})$, 3068 (C-H), 2974 (C-H), 2929 (C-H), 1595, 1574, 1473, 1436, 1368, 1348, 1265, 1202, 1159, 1132, 1092, 1071, 1047, 1035, 1007. HRMS (EI): Calcd. $\mathrm{C}_{8} \mathrm{H}_{9} \mathrm{ClO} m / z=156.0337$, found $m / z=$ $156.0336[\mathrm{M}]^{+} .[\boldsymbol{\alpha}]_{\mathbf{D}}{ }^{20}:+65.1\left(\mathrm{c}=1.0, \mathrm{CHCl}_{3}\right) . \mathbf{R}_{\mathbf{f}}\left(\mathrm{Et}_{2} \mathrm{O}:\right.$ pentane $\left.=1: 3\right)=0.37 . \mathbf{G C}: \beta-\mathrm{DEX}$ column, $130{ }^{\circ} \mathrm{C}$ isotherm, retention times $t_{\mathrm{R}}($ major $)=17.7 \mathrm{~min}, t_{\mathrm{R}}($ minor $)=21.2 \mathrm{~min}$. Analytical data are in agreement with literature data. ${ }^{54,5}$

Figure S59: GC trace of enantioenriched $(R)-11 \mathbf{c}$ (obtained with catalyst 9b).

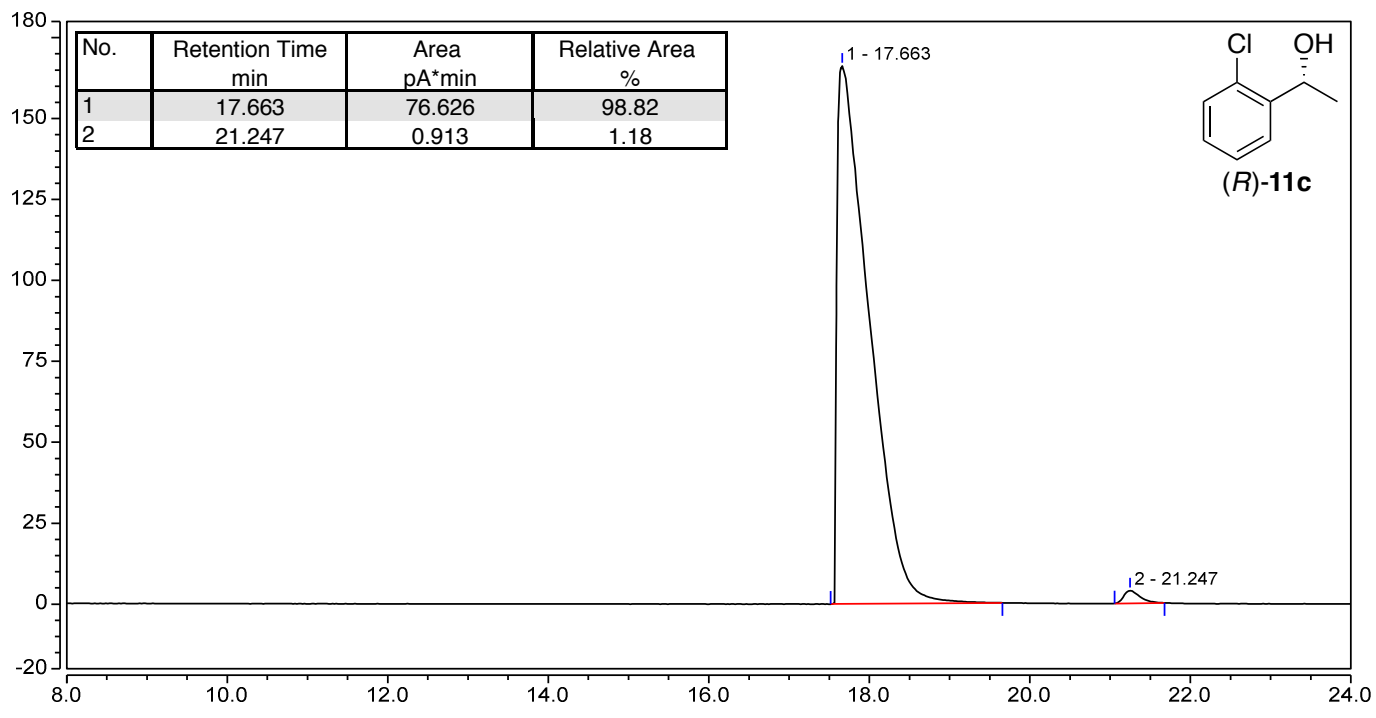


Figure S60: ${ }^{1} \mathrm{H}$ NMR spectrum of enantioenriched $(R)-11 \mathrm{c}\left(300 \mathrm{MHz}, \mathrm{CDCl}_{3}\right)$.

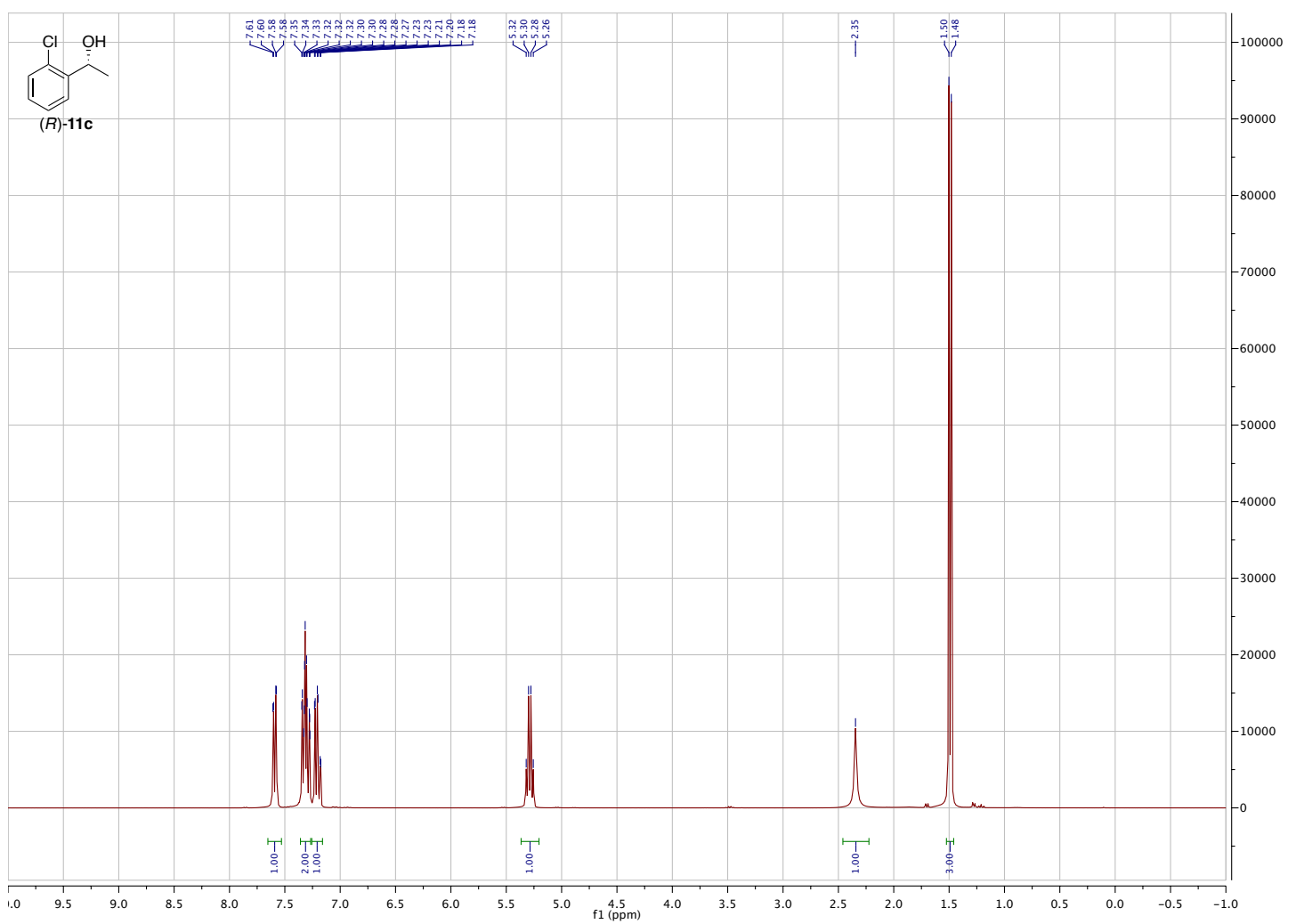

Figure S61: ${ }^{13} \mathrm{C}\left\{{ }^{1} \mathrm{H}\right\}$ NMR spectrum of enantioenriched $(R)-11 \mathrm{c}\left(75 \mathrm{MHz}, \mathrm{CDCl}_{3}\right)$.

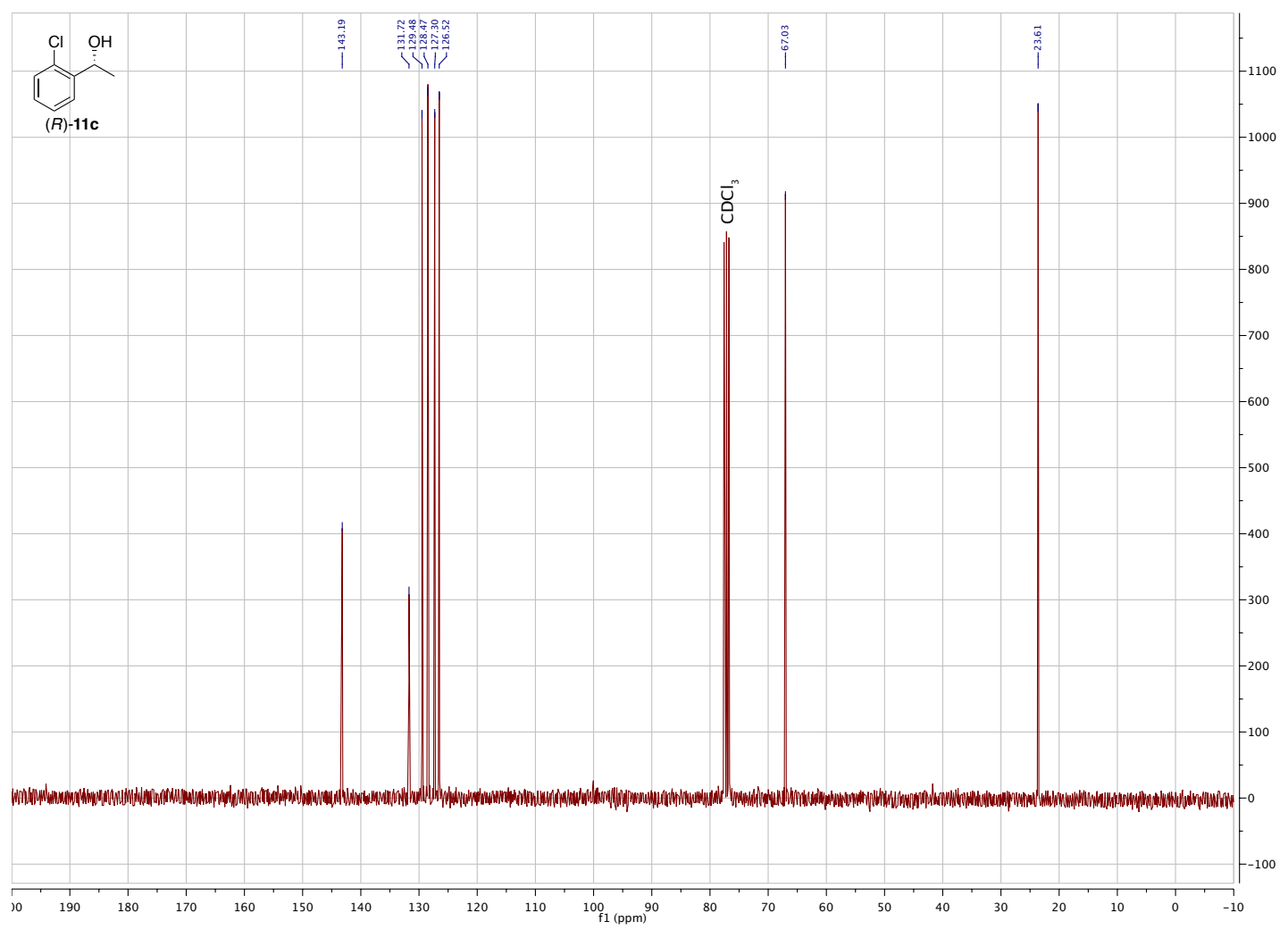




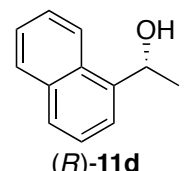

Synthesis of $(+)-(R)-1-(N a p h t h a l e n e-1-y l) e t h a n-1-o l, 11 d$. In a glove box, a vial was charged with complex $9 \mathbf{b}(40.7 \mathrm{mg}, 40.0 \mu \mathrm{mol}, 0.04 \mathrm{~mol} \%)$ and sodium tert-butoxide $(57.7 \mathrm{mg}$, $600 \mu \mathrm{mol}, 0.60 \mathrm{~mol} \%)$. The vial was closed with a plastic cap and removed from the glove box. The solids were added to a flame-dried $1000 \mathrm{~mL}$ two-neck round-bottom flask with reflux condenser using a paper funnel and dried for $5 \mathrm{~min}$ under high vacuum. 2-Propanol $(250 \mathrm{~mL})$ was added using a Teflon cannula, and the flask was immersed in a preheated oil bath at $75{ }^{\circ} \mathrm{C}$. After $5 \mathrm{~min}$, 2-propanol $(250 \mathrm{~mL})$ was added using a Teflon cannula. After additional $5 \mathrm{~min}$, 1-acetonaphthone (10d) $(15.2 \mathrm{~mL}, 100 \mathrm{mmol})$ was added, and the solution was stirred at $75{ }^{\circ} \mathrm{C}$ for $1.5 \mathrm{~h}$, when the reaction mixture showed almost full conversion. The solvent was removed at the rotary evaporator $\left(40{ }^{\circ} \mathrm{C}, 90\right.$ mbar), and the crude product was purified by flash column chromatography on silica gel $\left(\mathrm{Et}_{2} \mathrm{O}:\right.$ pentane $=0: 1$ to $\left.3: 2\right)$ to afford the product as a slightly yellowish liquid. Yield: $17.11 \mathrm{~g}(99.3 \%, 99.4 \% e e) .{ }^{1} \mathbf{H}$ NMR (300 MHz, $\left.\mathrm{CDCl}_{3}\right): \delta 8.14-8.03(\mathrm{~m}$, $1 \mathrm{H}, \operatorname{Ar}-H), 7.93-7.85(m, 1 \mathrm{H}, \mathrm{Ar}-H), 7.79\left(d,{ }^{3} J_{\mathrm{H}_{\mathrm{H}}}{ }^{\prime}=8.2 \mathrm{~Hz}, 1 \mathrm{H}, \operatorname{Ar}-H\right), 7.67\left(d,{ }^{3} J_{\mathrm{H}, \mathrm{H}^{\prime}}=7.1 \mathrm{~Hz}\right.$, $1 \mathrm{H}, \operatorname{Ar}-H), 7.56-7.44(m, 3 \mathrm{H}, \mathrm{Ar}-H), 5.62\left(q,{ }^{3} J_{\mathrm{H}, \mathrm{H}^{\prime}}=6.5 \mathrm{~Hz}, 1 \mathrm{H}, \mathrm{CHOH}\right), 2.33($ br $s, 1 \mathrm{H}, \mathrm{OH})$, $1.66\left(d,{ }^{3} J_{\mathrm{H}, \mathrm{H}^{\prime}}=6.5 \mathrm{~Hz}, 3 \mathrm{H}, \mathrm{CH}\right) .{ }^{13} \mathbf{C}\left\{{ }^{1} \mathbf{H}\right\} \mathbf{N M R}\left(75 \mathrm{MHz}, \mathrm{CDCl}_{3}\right): \delta 141.5$ (arom.), 133.9 (arom.), 130.3 (arom.), 128.9 (arom.), 127.9 (arom.), 126.1 (arom.), 125.6 (s, 2C, arom.), 123.2 (arom.), 122.1 (arom.), $67.1(\mathrm{CHOH}), 24.4\left(\mathrm{CH}_{3}\right)$. IR (liquid film, $\left.\mathrm{cm}^{-1}\right)$ : $3324(\mathrm{O}-\mathrm{H}), 3049(\mathrm{C}-\mathrm{H})$, 2971 (C-H), 2926 (C-H), 1596, 1509, 1443, 1394, 1368, 1324, 1260, 1230, 1206, 1168, 1108, 1063 , 1030, 1010. HRMS (EI): Calcd. for $\mathrm{C}_{12} \mathrm{H}_{12} \mathrm{O} m / z=172.0883$, found $m / z=172.0887[\mathrm{M}]^{+}$. $[\boldsymbol{\alpha}]_{\mathbf{D}}{ }^{20}$ : $+67.7\left(\mathrm{c}=1.0, \mathrm{CHCl}_{3}\right) . \mathbf{R}_{\mathbf{f}}\left(\mathrm{Et}_{2} \mathrm{O}:\right.$ pentane $\left.=1: 3\right)=0.32 . \mathbf{G C}: \beta-\mathrm{DEX}$ column, $165{ }^{\circ} \mathrm{C}$ isotherm, retention times $t_{\mathrm{R}}($ minor $)=29.7 \mathrm{~min}, t_{\mathrm{R}}($ major $)=30.4 \mathrm{~min}$. Analytical data are in agreement with literature data. ${ }^{\mathrm{S}, 5}$ 
Figure S62: ${ }^{1} \mathrm{H}$ NMR spectrum of enantioenriched $(R)-\mathbf{1 1 d}\left(300 \mathrm{MHz}, \mathrm{CDCl}_{3}\right)$.

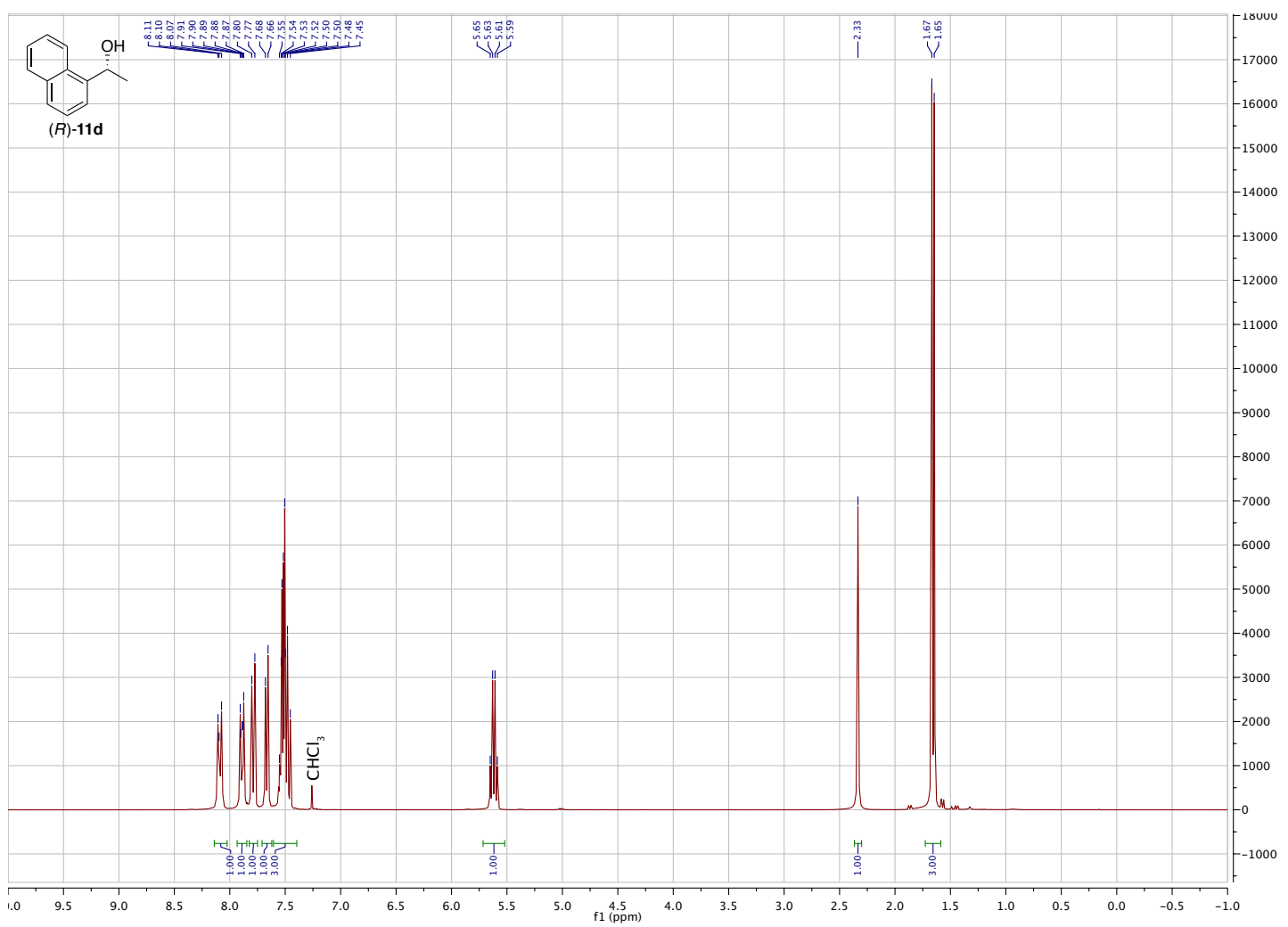

Figure S63: ${ }^{13} \mathrm{C}\left\{{ }^{1} \mathrm{H}\right\}$ NMR spectrum of enantioenriched $(R)$-11d $\left(75 \mathrm{MHz}, \mathrm{CDCl}_{3}\right)$.

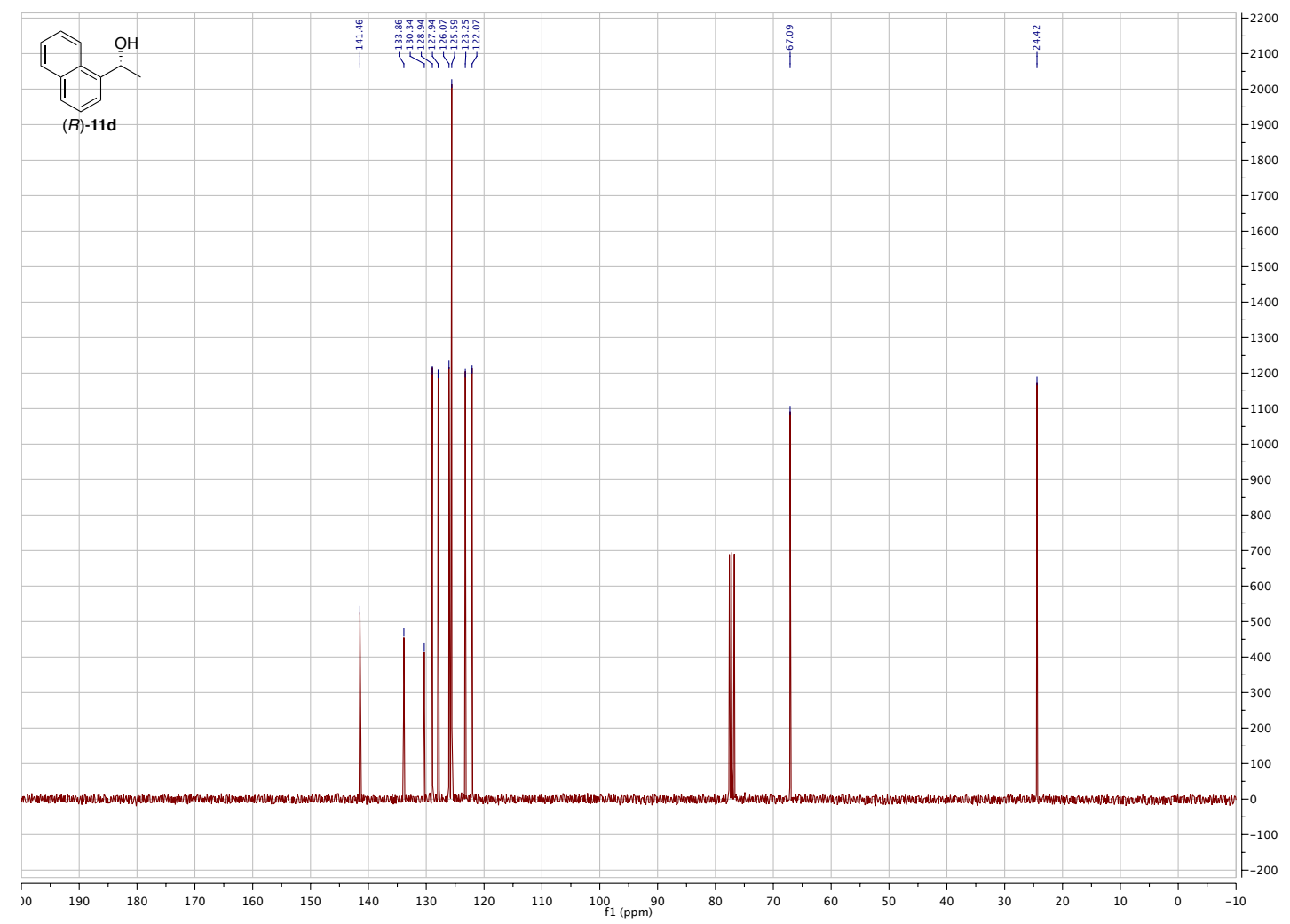


Figure S64: GC trace of enantioenriched $(R)-11 d$ (obtained with catalyst $9 \mathbf{b}$ ).
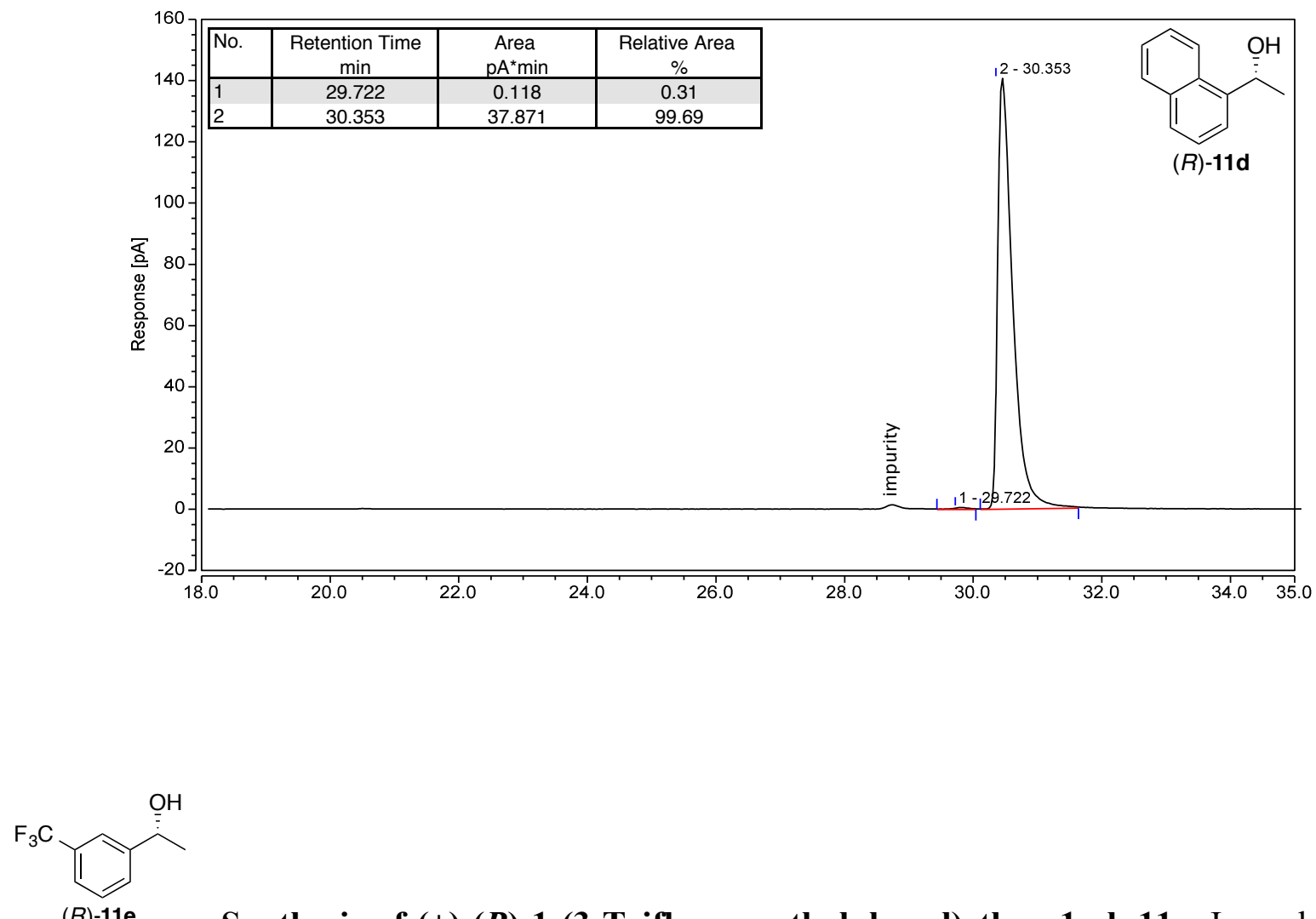

Synthesis of (+)-(R)-1-(3-Trifluoromethylphenyl)ethan-1-ol, 11e. In a glove box, a vial was charged with complex 9a $(67.8 \mathrm{mg}, 66.7 \mu \mathrm{mol}, 0.0667 \mathrm{~mol} \%)$ and sodium tert-butoxide (64.1 $\mathrm{mg}, 667 \mu \mathrm{mol}, 0.667 \mathrm{~mol} \%$ ). The vial was closed with a plastic cap and removed from the glove box. The solids were added to a flame-dried $1000 \mathrm{~mL}$ two-neck round-bottom flask using a paper funnel and dried for 5 min under high vacuum. 2-Propanol $(250 \mathrm{~mL})$ was added using a Teflon cannula, and the flask was immersed in a preheated oil bath at $60{ }^{\circ} \mathrm{C}$. After 5 min, 2-propanol $(250 \mathrm{~mL})$ was added using a Teflon cannula. After additional $5 \mathrm{~min}$, 3-trifluoromethylacetophenone $(\mathbf{1 0 e})(15.3 \mathrm{~mL}, 100 \mathrm{mmol})$ was added, and the solution was stirred at $60^{\circ} \mathrm{C}$ for $1.0 \mathrm{~h}$, when the reaction mixture showed almost full conversion. The solvent was removed quickly at the rotary evaporator $\left(40{ }^{\circ} \mathrm{C}, 80 \mathrm{mbar}\right)$, and the crude product was purified by flash column chromatography on silica gel $\left(\mathrm{Et}_{2} \mathrm{O}:\right.$ pentane $=1: 9$ to $\left.1: 1\right)$ to afford the product as a slightly yellowish liquid. Yield: 18.96 g $(99.7 \%, 97.1 \%$ ee $) .{ }^{1} \mathbf{H}$ NMR (300 MHz, $\left.\mathrm{CDCl}_{3}\right): \delta 7.64$ (br $\left.s, 1 \mathrm{H}, \mathrm{Ar}-H\right)$, 
$7.57-7.50(m, 2 \mathrm{H}, \mathrm{Ar}-H), 7.49-7.42(m, 1 \mathrm{H}, \mathrm{A}--H), 4.94\left(q,{ }^{3} J_{\mathrm{H}, \mathrm{H}^{\prime}}=6.5 \mathrm{~Hz}, 1 \mathrm{H}, \mathrm{CHOH}\right)$, 2.14 (br $s, 1 \mathrm{H}, \mathrm{OH}), 1.50\left(d,{ }^{3} J_{\mathrm{H}, \mathrm{H}}=6.5 \mathrm{~Hz}, 3 \mathrm{H}, \mathrm{CH}_{3}\right) .{ }^{13} \mathbf{C}\left\{{ }^{1} \mathbf{H}\right\} \mathbf{N M R}\left(75 \mathrm{MHz}, \mathrm{CDCl}_{3}\right): \delta 146.8$ (arom.), $130.9\left(q,{ }^{2} J_{\mathrm{C}, \mathrm{F}}=32.2 \mathrm{~Hz}\right.$, arom.), 129.0 (arom.), $128.9\left(q,{ }^{4} J_{\mathrm{C}, \mathrm{F}}=1.4 \mathrm{~Hz}\right.$, arom.), $124.33(q$, ${ }^{3} J_{\mathrm{C}, \mathrm{F}}=3.8 \mathrm{~Hz}$, arom. $), 124.30\left(q,{ }^{1} J_{\mathrm{C}, \mathrm{F}}=272.3 \mathrm{~Hz}, C \mathrm{~F}_{3}\right), 122.3\left(q,{ }^{3} J_{\mathrm{C}, \mathrm{F}}=3.8 \mathrm{~Hz}\right.$, arom. $), 69.9$ $(\mathrm{CHOH}), 25.4\left(\mathrm{CH}_{3}\right) .{ }^{19} \mathbf{F}\left\{{ }^{1} \mathbf{H}\right\}$ NMR $\left(282 \mathrm{MHz}, \mathrm{CDCl}_{3}\right): \delta-62.6(s)$. IR (liquid film, $\left.\mathrm{cm}^{-1}\right)$ : 3325 (O-H), 2977 (C-H), 2931 (C-H), 2891 (C-H), 1493, 1451, 1407, 1373, 1326, 1269, 1195, 1161, 1117, 1069, 1012. HRMS (EI): Calcd. for $\mathrm{C}_{9} \mathrm{H}_{9} \mathrm{~F}_{3} \mathrm{O} \mathrm{m} / \mathrm{z}=190.0600$, found $\mathrm{m} / \mathrm{z}=$ $190.0600[\mathrm{M}]^{+} .[\boldsymbol{\alpha}]_{\mathbf{D}}{ }^{\mathbf{2 0}}:+34.2\left(\mathrm{c}=1.0, \mathrm{CHCl}_{3}\right) . \mathbf{R}_{\mathbf{f}}\left(\mathrm{Et}_{2} \mathrm{O}:\right.$ pentane $\left.=1: 3\right)=0.48 . \mathbf{G C}: \beta-\mathrm{DEX}$ column, $90{ }^{\circ} \mathrm{C}$ isotherm, retention times $t_{\mathrm{R}}$ (major) $=40.6 \mathrm{~min}, t_{\mathrm{R}}($ minor $)=45.8 \mathrm{~min}$. Analytical data are in agreement with literature data. ${ }^{\mathrm{S} 4}$

Figure S65: ${ }^{1} \mathrm{H}$ NMR spectrum of enantioenriched $(R)-11 e\left(300 \mathrm{MHz}, \mathrm{CDCl}_{3}\right)$.

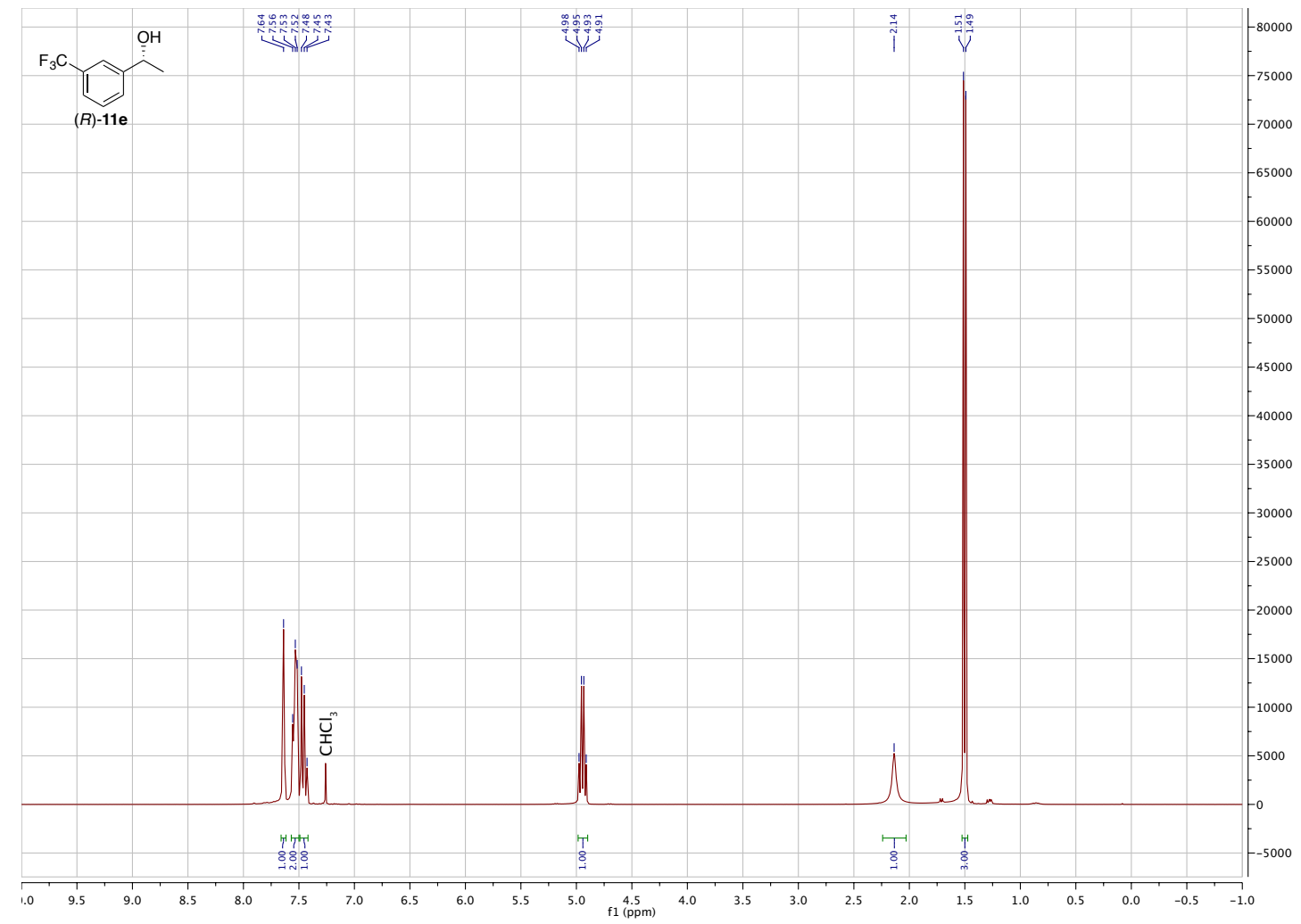


Figure S66: ${ }^{13} \mathrm{C}\left\{{ }^{1} \mathrm{H}\right\}$ NMR spectrum of enantioenriched $(R)-11 e\left(75 \mathrm{MHz}, \mathrm{CDCl}_{3}\right)$.

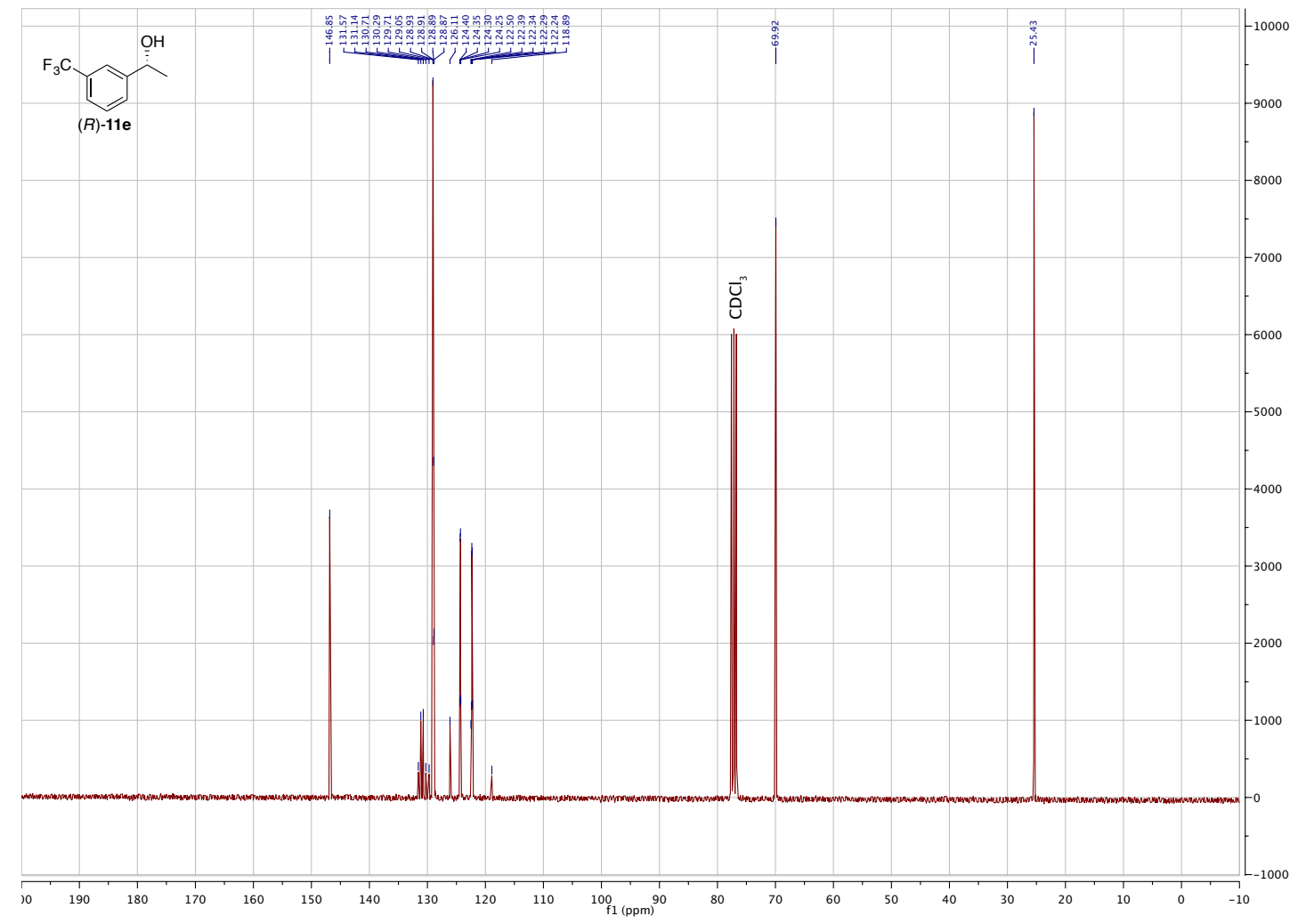

Figure S67: ${ }^{19} \mathrm{~F}\left\{{ }^{1} \mathrm{H}\right\}$ NMR spectrum of enantioenriched $(R)-11 e\left(282 \mathrm{MHz}, \mathrm{CDCl}_{3}\right)$.

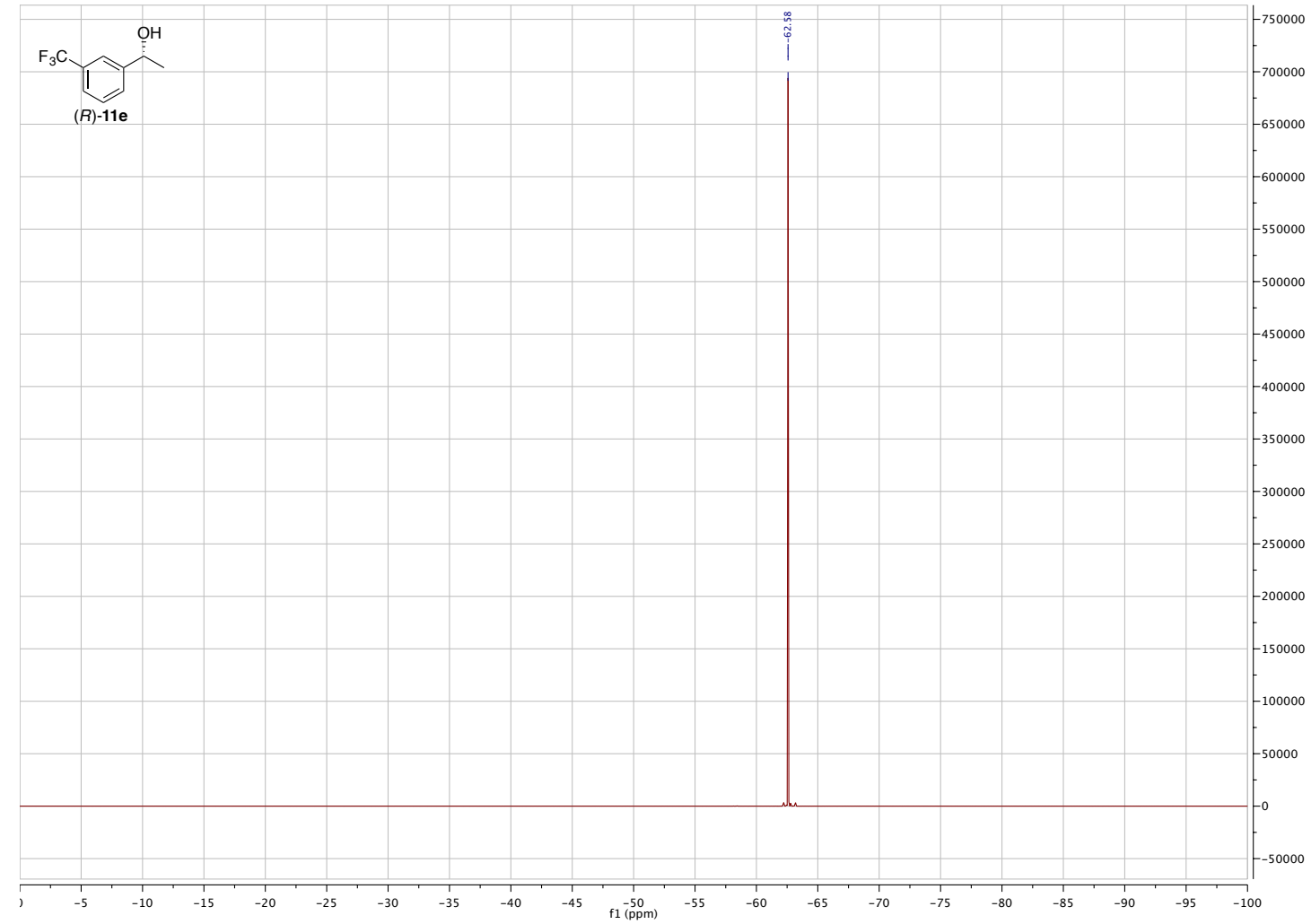


Figure S68: GC trace of enantioenriched $(R)-11 \mathrm{e}$ (obtained with catalyst 9a).
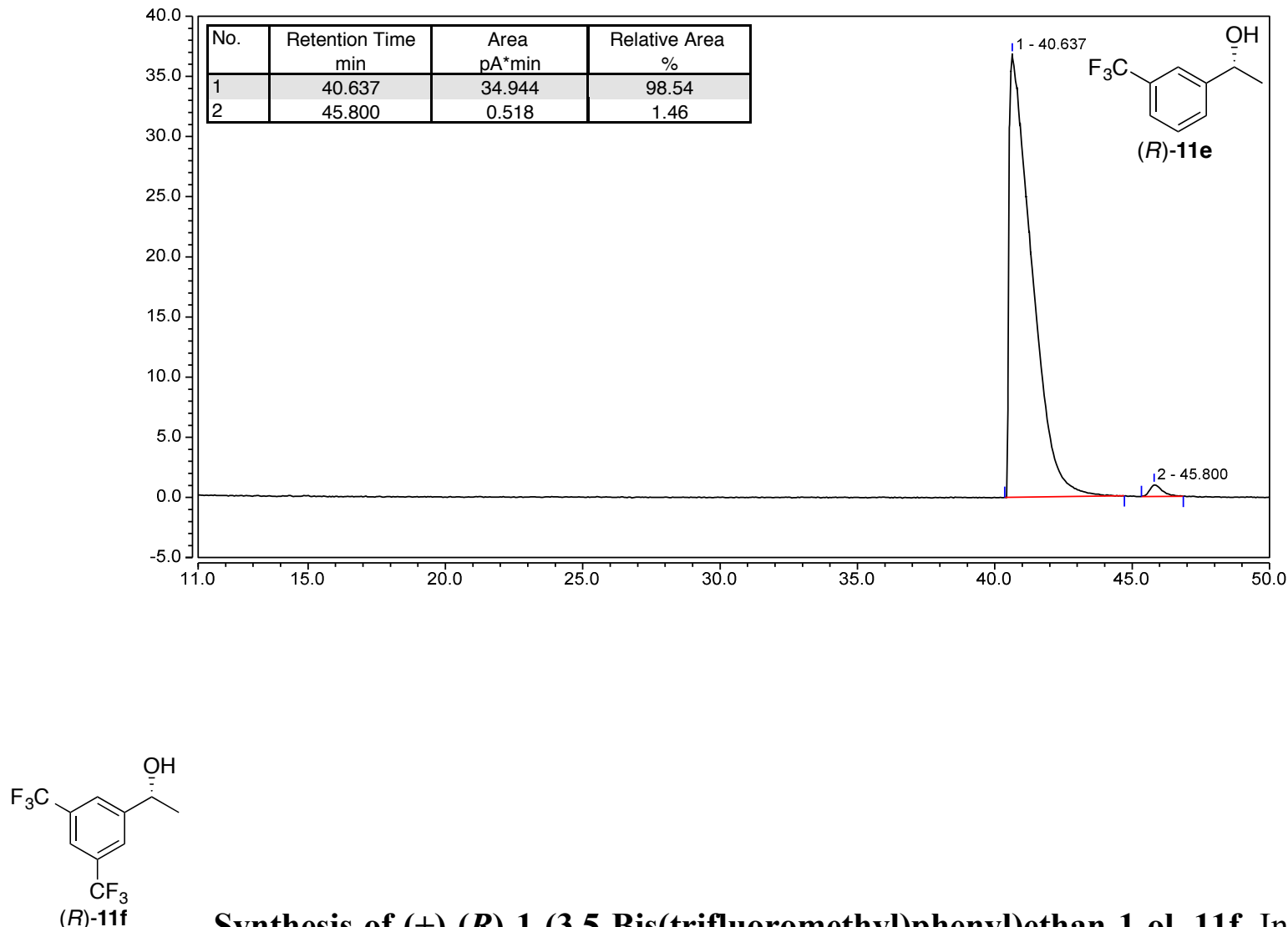

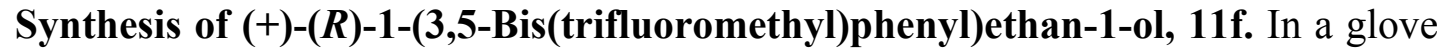

box, a vial was charged with complex 9a $(20.3 \mathrm{mg}, 40.0 \mu \mathrm{mol}, 0.02 \mathrm{~mol} \%)$ and sodium tertbutoxide (38.4 mg, $40.0 \mu \mathrm{mol}, 0.4 \mathrm{~mol} \%)$. The vial was closed with a plastic cap and removed from the glove box. The solids were added to a flame-dried $1000 \mathrm{~mL}$ two-neck round-bottom flask using a paper funnel and dried for 5 min under high vacuum. 2-Propanol $(250 \mathrm{~mL})$ was added using a Teflon cannula, and the flask was immersed in a preheated oil bath at $60{ }^{\circ} \mathrm{C}$. After $5 \mathrm{~min}$, 2-propanol $(250 \mathrm{~mL})$ was added using a Teflon cannula. After additional $5 \mathrm{~min}$, 3,5-bis(trifluoromethyl)acetophenone $(\mathbf{1 0 f})(18.0 \mathrm{~mL}, 100 \mathrm{mmol})$ was added, and the solution was stirred at $60{ }^{\circ} \mathrm{C}$ for $1.5 \mathrm{~h}$, when the reaction mixture showed almost full conversion. The solvent was removed at the rotary evaporator $\left(40{ }^{\circ} \mathrm{C}, 90 \mathrm{mbar}\right)$, and the crude product was purified by flash column chromatography on silica gel $\left(\mathrm{Et}_{2} \mathrm{O}:\right.$ pentane $=1: 9$ to $\left.1: 1\right)$ to afford the title product as a white solid. Yield: $25.45 \mathrm{~g}\left(98.6 \%, 99.1 \%\right.$ ee) ${ }^{1} \mathbf{H}$ NMR (300 MHz, $\left.\mathrm{CDCl}_{3}\right): \delta 7.72(\mathrm{br} s, 2 \mathrm{H}$, 
$\operatorname{Ar}-H), 7.68(\mathrm{br} s, 1 \mathrm{H}, \mathrm{Ar}-H), 5.03\left(q d,{ }^{3} J_{\mathrm{H}, \mathrm{H}^{\prime}}=6.5,3.3 \mathrm{~Hz}, 1 \mathrm{H}, \mathrm{CHOH}\right), 2.60\left(\mathrm{br} d,{ }^{3} J_{\mathrm{H}, \mathrm{H}^{\prime}}=3.3 \mathrm{~Hz}\right.$, $1 \mathrm{H}, \mathrm{OH}), 1.42\left(d,{ }^{3} J_{\mathrm{H}, \mathrm{H}^{\prime}}=6.5 \mathrm{~Hz}, 3 \mathrm{H}, \mathrm{CH}_{3}\right) .{ }^{13} \mathbf{C}\left\{{ }^{1} \mathbf{H}\right\} \mathbf{N M R}\left(75 \mathrm{MHz}, \mathrm{CDCl}_{3}\right): \delta 148.3$ (arom.), $131.9\left(q,{ }^{2} J_{\mathrm{C}, \mathrm{F}}=33.3 \mathrm{~Hz}\right.$, arom.), $125.8(m$, arom. $), 123.5\left(q,{ }^{1} J_{\mathrm{C}, \mathrm{F}}=272.6 \mathrm{~Hz}, C \mathrm{~F}_{3}\right), 121.5($ sept, ${ }^{3} J_{\mathrm{C}, \mathrm{F}}=3.9 \mathrm{~Hz}$, arom.), $69.4(\mathrm{CHOH}), 25.5\left(\mathrm{CH}_{3}\right) .{ }^{19} \mathbf{F}\left\{{ }^{1} \mathbf{H}\right\}$ NMR $\left(282 \mathrm{MHz}, \mathrm{CDCl}_{3}\right): \delta-63.1(s)$. IR (solid, cm ${ }^{-1}$ ): 3274 (O-H), 2980 (C-H), 2929 (C-H), 2911 (C-H), 2883 (C-H), 1623, 1466, 1460, $1430,1376,1360,1338,1321,1274,1197,1163,1133,1113,1075,1028,1012$. HRMS (EI): Calcd. for $\mathrm{C}_{10} \mathrm{H}_{8} \mathrm{~F}_{6} \mathrm{O} m / z=258.0474$, found $m / z=258.0471[\mathrm{M}]^{+}$. Melting Point: $54{ }^{\circ} \mathrm{C}$. $[\boldsymbol{\alpha}]_{\mathbf{D}}{ }^{20}:+22.9\left(\mathrm{c}=1.0, \mathrm{CHCl}_{3}\right) \cdot \mathbf{R}_{\mathbf{f}}\left(\mathrm{Et}_{2} \mathrm{O}:\right.$ pentane $\left.=1: 3\right)=0.34 . \mathbf{G C}: \beta-\mathrm{DEX}$ column, $100{ }^{\circ} \mathrm{C}$ isotherm, retention times $t_{\mathrm{R}}($ minor $)=13.6 \mathrm{~min}, t_{\mathrm{R}}($ major $)=14.9 \mathrm{~min}$. Analytical data are in agreement with literature data. ${ }^{\mathrm{S} 4}$

Figure S69: ${ }^{1} \mathrm{H}$ NMR spectrum of enantioenriched $(R)-11 f\left(300 \mathrm{MHz}, \mathrm{CDCl}_{3}\right)$.

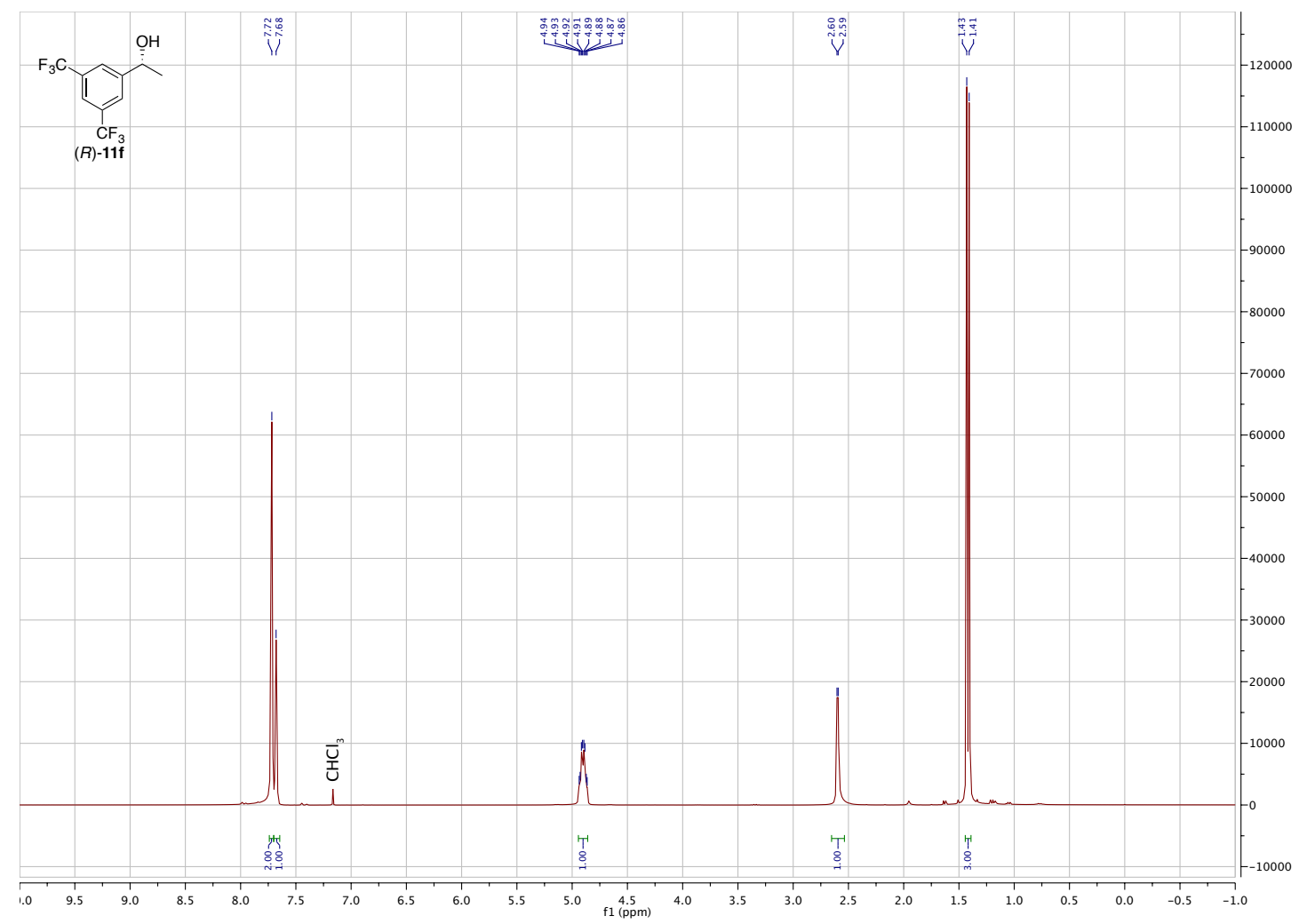


Figure S70: ${ }^{13} \mathrm{C}\left\{{ }^{1} \mathrm{H}\right\}$ NMR spectrum of enantioenriched $(R)-11 f\left(75 \mathrm{MHz}, \mathrm{CDCl}_{3}\right)$.

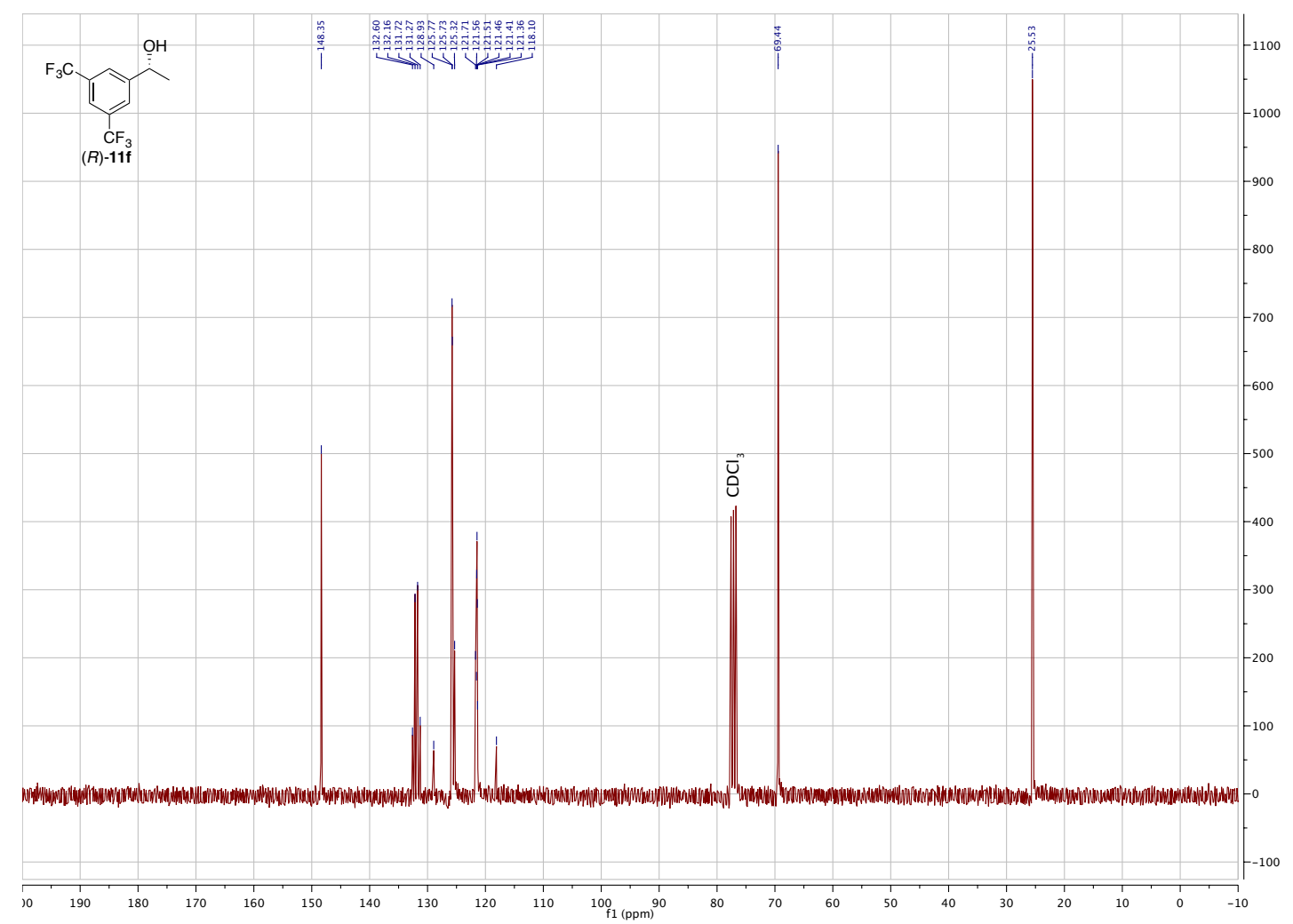

Figure S71: ${ }^{19} \mathrm{~F}\left\{{ }^{1} \mathrm{H}\right\}$ NMR spectrum of enantioenriched $(R)$-11f $\left(282 \mathrm{MHz}, \mathrm{CDCl}_{3}\right)$.

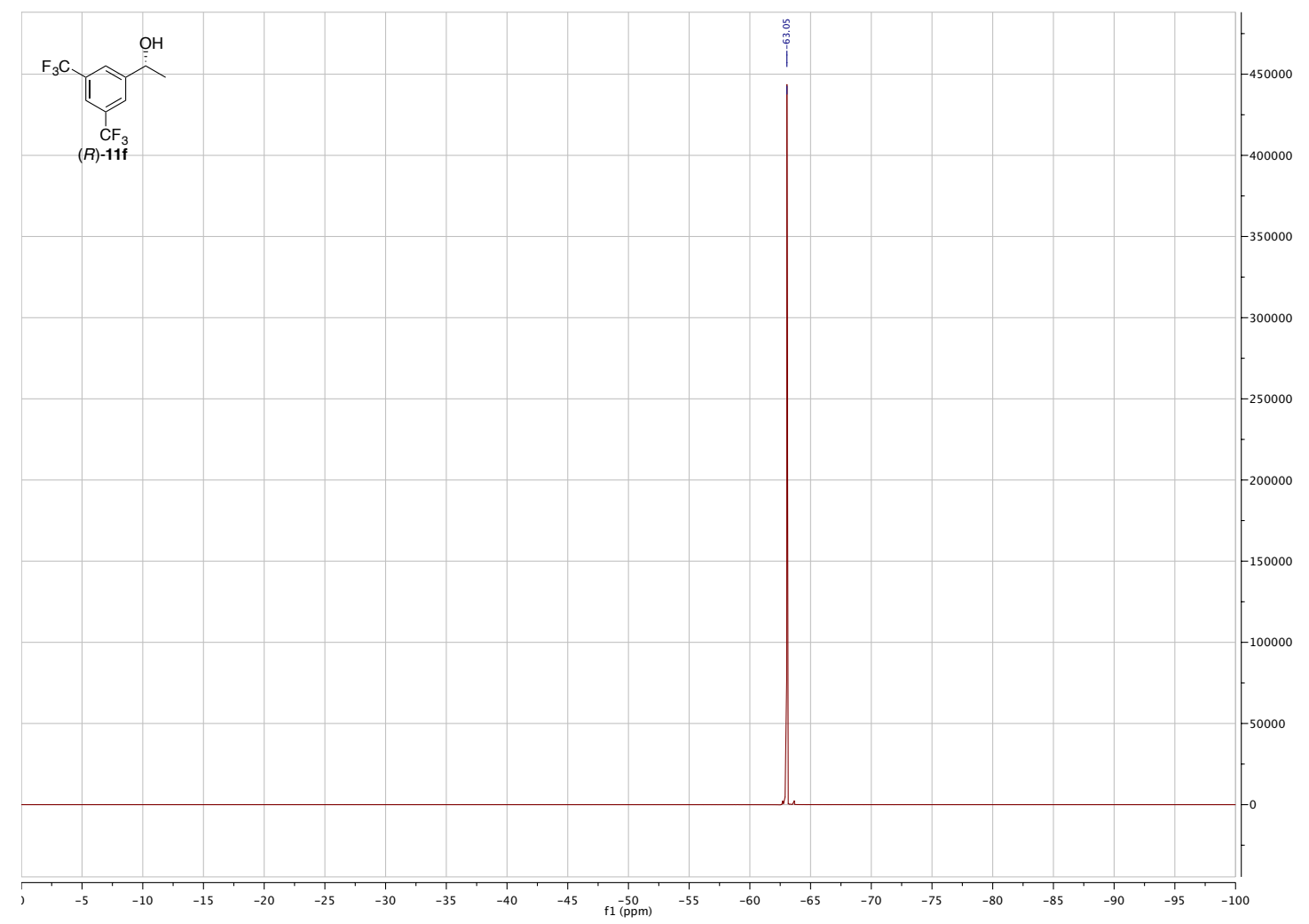


Figure S72: GC trace of enantioenriched $(R)-\mathbf{1 1 f}$ (obtained with catalyst 9a).
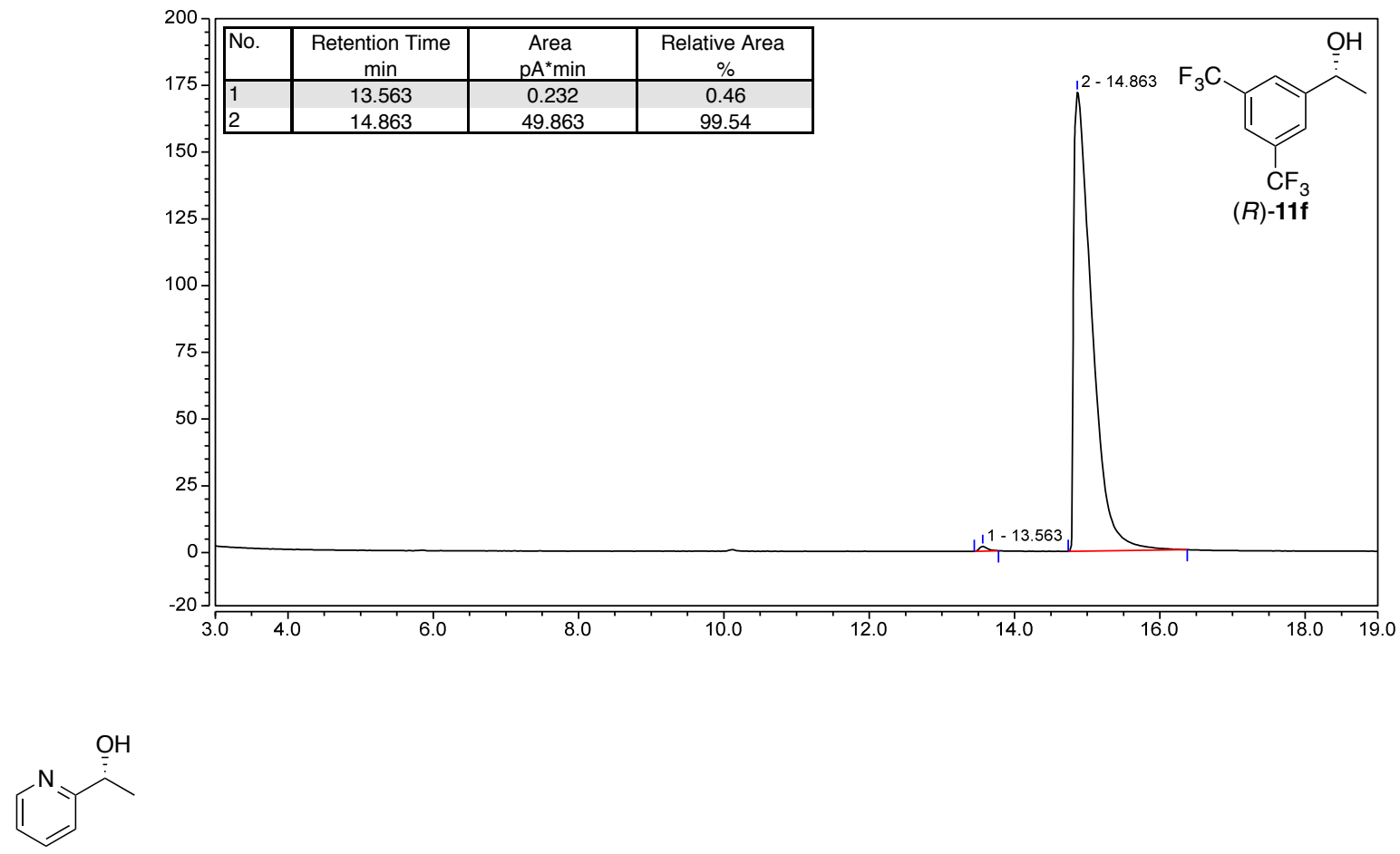

$(R)-\mathbf{1 1 g}$

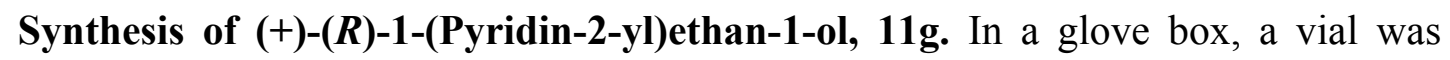
charged with complex 9a $(10.2 \mathrm{mg}, 10.0 \mu \mathrm{mol}, 0.01 \mathrm{~mol} \%)$ and sodium tert-butoxide (24.0 $\mathrm{mg}$, $250 \mu \mathrm{mol}, 0.25 \mathrm{~mol} \%)$. The vial was closed with a plastic cap and removed from the glove box. The solids were added to a flame-dried $1000 \mathrm{~mL}$ two-neck round-bottom flask using a paper funnel and dried for 5 min under high vacuum. 2-Propanol $(250 \mathrm{~mL})$ was added using a Teflon cannula, and the flask was immersed in a preheated oil bath at $60{ }^{\circ} \mathrm{C}$. After $5 \mathrm{~min}$, 2-propanol $(250 \mathrm{~mL})$ was added using a Teflon cannula. After additional $5 \mathrm{~min}$, 2-acetylpyridine $(\mathbf{1 0 g})(11.2 \mathrm{~mL}, 100 \mathrm{mmol})$ was added, and the solution was stirred at $60^{\circ} \mathrm{C}$ for $1.5 \mathrm{~h}$, when the reaction mixture showed almost full conversion (99.8\%). The solvent was removed quickly at the rotary evaporator to prevent racemization $\left(40{ }^{\circ} \mathrm{C}, 70 \mathrm{mbar}\right)$. The crude product was dissolved in $\mathrm{Et}_{2} \mathrm{O}(100 \mathrm{~mL})$ and filtered through a short Celite ${ }^{\circledR}$ pad. The pad was washed three times with $\mathrm{Et}_{2} \mathrm{O}(3 \times 30 \mathrm{~mL})$ and the solvent was removed at the rotary evaporator $\left(40{ }^{\circ} \mathrm{C}, 50 \mathrm{mbar}\right)$. The crude product was purified by distillation $\left(52{ }^{\circ} \mathrm{C}, 1.0 \times 10^{-1}\right.$ mbar $)$ to afford the product a clear liquid that solidified upon 
standing. Yield: $11.96 \mathrm{~g}(97.1 \%, 97.3 \% e e) .{ }^{1} \mathbf{H}$ NMR $\left(300 \mathrm{MHz}, \mathrm{CDCl}_{3}\right): \delta 8.43\left(d d d,{ }^{3} \mathrm{~J}_{\mathrm{H}, \mathrm{H}}=\right.$ $\left.4.9 \mathrm{~Hz},{ }^{4} J_{\mathrm{H}, \mathrm{H}^{\prime}}=1.8 \mathrm{~Hz},{ }^{5} J_{\mathrm{H}, \mathrm{H}^{\prime}}=1.0 \mathrm{~Hz}, 1 \mathrm{H}, \mathrm{Ar}-H\right), 7.60\left(d d d,{ }^{3} J_{\mathrm{H}, \mathrm{H}^{\prime}}=7.8,7.6 \mathrm{~Hz},{ }^{4} J_{\mathrm{H}, \mathrm{H}^{\prime}}=1.8 \mathrm{~Hz}\right.$, $1 \mathrm{H}, \operatorname{Ar}-H), 7.22\left(\mathrm{br} d,{ }^{3} J_{\mathrm{H}, \mathrm{H}^{\prime}}=7.6 \mathrm{~Hz}, 1 \mathrm{H}, \operatorname{Ar}-H\right), 7.13-7.07(m, 1 \mathrm{H}, \operatorname{Ar}-H), 4.81\left(q,{ }^{3} J_{\mathrm{H}, \mathrm{H}^{\prime}}=\right.$ $6.5 \mathrm{~Hz}, 1 \mathrm{H}, \mathrm{CHOH}), 4.40($ br $s, 1 \mathrm{H}, \mathrm{OH}), 1.42\left(d,{ }^{3} J_{\mathrm{H}, \mathrm{H}^{\prime}}=6.5 \mathrm{~Hz}, 3 \mathrm{H}, \mathrm{CH}_{3}\right) .{ }^{13} \mathbf{C}\left\{{ }^{1} \mathbf{H}\right\} \mathbf{N M R}$ (75 MHz, $\mathrm{CDCl}_{3}$ ): $\delta 163.3$ (arom.), 148.2 (arom.), 136.9 (arom.), 122.3 (arom.), 119.9 (arom.), 69.1 (CHOH), $24.3\left(\mathrm{CH}_{3}\right)$. IR (liquid film, $\left.\mathrm{cm}^{-1}\right)$ : $3254(\mathrm{O}-\mathrm{H}), 3067(\mathrm{C}-\mathrm{H}), 3012(\mathrm{C}-\mathrm{H}), 2973(\mathrm{C}-\mathrm{H})$, 2927 (C-H), 2874 (C-H), 1595, 1571, 1475, 1451, 1435, 1403, 1365, 1337, 1298, 1220, 1150, 1117, 1081, 1048, 1015. HRMS (EI): Calcd. for $\mathrm{C}_{7} \mathrm{H}_{9} \mathrm{NO} m / z=123.0679$, found $m / z=123.0680[\mathrm{M}]^{+}$. $[\alpha]_{\mathbf{D}}{ }^{20}:+26.6\left(\mathrm{c}=1.0, \mathrm{CHCl}_{3}\right)$. HPLC: OD-H (hexane $: 2-\mathrm{PrOH}=99: 1$, flow rate $0.8 \mathrm{~mL} / \mathrm{min}, \lambda=$ $210 \mathrm{~nm}$ ), retention times $t_{\mathrm{R}}($ major $)=24.0 \mathrm{~min}, t_{\mathrm{R}}($ minor $)=27.3 \mathrm{~min}$. Analytical data are in agreement with literature data. ${ }^{\mathrm{S}}$

Figure S73: ${ }^{1} \mathrm{H}$ NMR spectrum of enantioenriched $(R)-\mathbf{1 1 g}\left(300 \mathrm{MHz}, \mathrm{CDCl}_{3}\right)$.

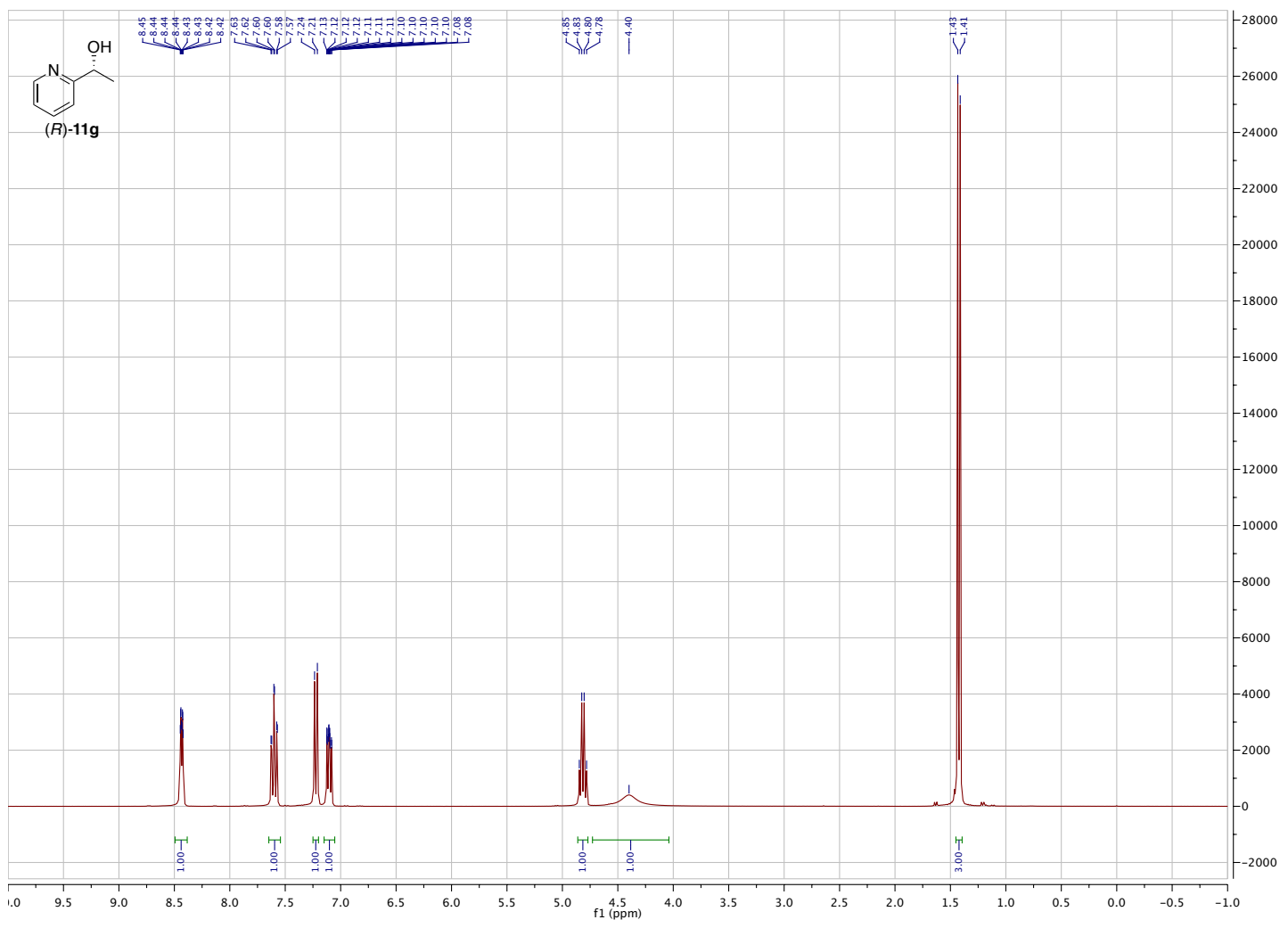


Figure S74: ${ }^{13} \mathrm{C}\left\{{ }^{1} \mathrm{H}\right\}$ NMR spectrum of enantioenriched $(R)-11 g\left(75 \mathrm{MHz}, \mathrm{CDCl}_{3}\right)$.

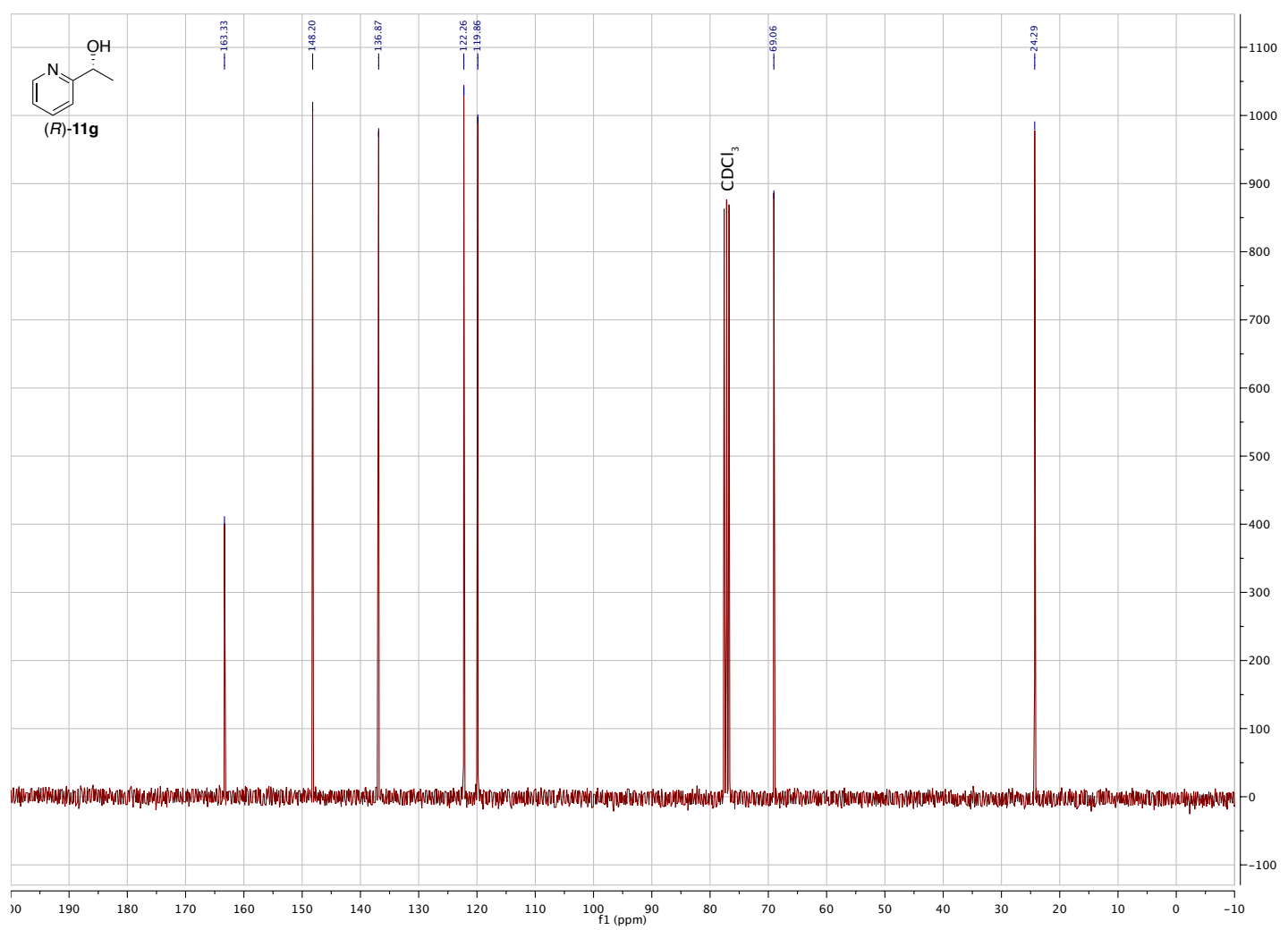

Figure S75: HPLC trace of enantioenriched $(R)-11$ (obtained with catalyst 9a).

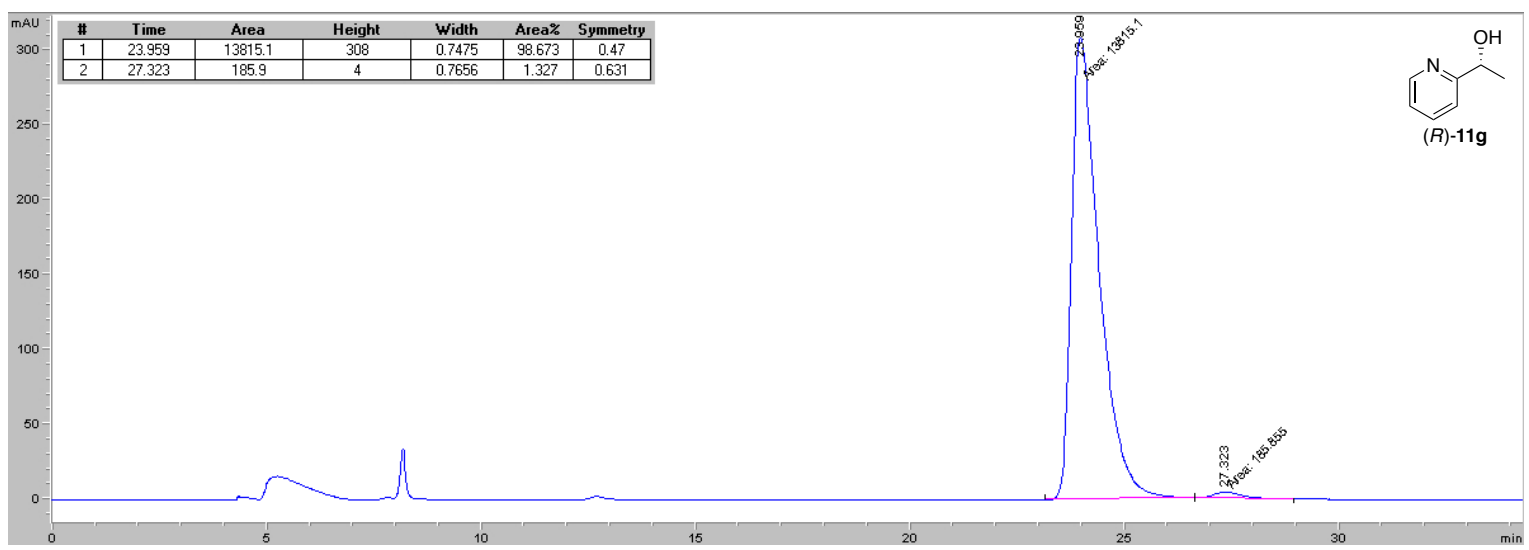


Figure S76: HPLC trace of racemic 11g.
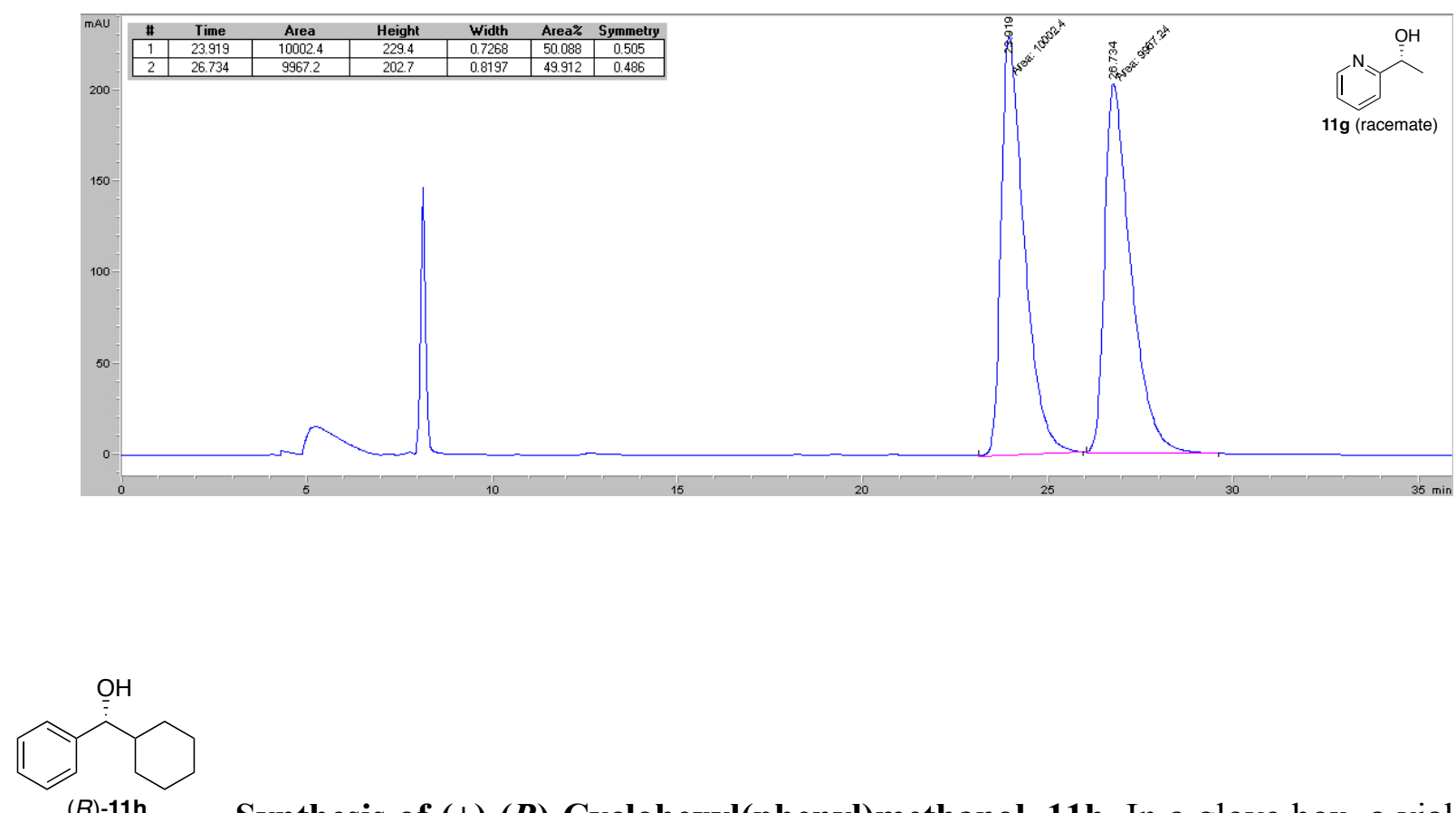

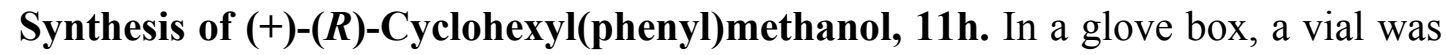
charged with complex 9b (40.7 mg, $10.0 \mu \mathrm{mol}, 0.04 \mathrm{~mol} \%$ ) and sodium tert-butoxide (57.7 mg, $250 \mu \mathrm{mol}, 0.6 \mathrm{~mol} \%$ ). The vial was closed with a plastic cap and removed from the glove box. The solids were added to a flame-dried $1000 \mathrm{~mL}$ two-neck round-bottom flask with reflux condenser using a paper funnel and dried for 5 min under high vacuum. 2-Propanol (250 mL) was added using a Teflon cannula and the flask was immersed in a preheated oil bath at $75{ }^{\circ} \mathrm{C}$. After 5 min, 2-propanol $(250 \mathrm{~mL})$ was added using a Teflon cannula. After additional 5 min, cyclohexyl(phenyl)methanone (10h) $(18.83 \mathrm{~g}, 100 \mathrm{mmol})$ was added, and the solution was stirred at $75^{\circ} \mathrm{C}$ for $5.0 \mathrm{~h}$. The solvent was removed at the rotary evaporator $\left(40^{\circ} \mathrm{C}, 90 \mathrm{mbar}\right)$, and the crude product was purified by flash column chromatography on silica gel (EtOAc : hexane $=0: 100$ to $1: 1$ ) to afford the title product as an off-white solid. Yield: $16.93 \mathrm{~g}(89.0 \%, 97.7 \%$ ee $) .{ }^{1} \mathbf{H}$ NMR $\left(300 \mathrm{MHz}, \mathrm{CDCl}_{3}\right): \delta 7.29-7.14(m, 5 \mathrm{H}, \mathrm{Ar}-H), 4.26\left(d,{ }^{3} J_{\mathrm{H}, \mathrm{H}}=7.1 \mathrm{~Hz}, 1 \mathrm{H}, \mathrm{CHOH}\right), 2.95-1.83$ $(m, 2 \mathrm{H}, \mathrm{CHCHOH}+\mathrm{OH}), 1.74-1.64(m, 1 \mathrm{H}, \mathrm{CHH}), 1.63-1.46(m, 3 \mathrm{H}, \mathrm{CHH}), 1.34-1.24(m$, $1 \mathrm{H}, \mathrm{CHH}), 1.22-0.77(m, 5 \mathrm{H}, \mathrm{CHH}) .{ }^{13} \mathbf{C}\left\{{ }^{1} \mathbf{H}\right\}$ NMR (75 MHz, $\left.\mathrm{CDCl}_{3}\right): \delta 143.8$ (arom.), 128.3 
(arom.), 127.5 (arom.), 126.8 (arom.), $79.5(\mathrm{CHOH}), 45.1(\mathrm{CHCHOH}), 29.5\left(\mathrm{CH}_{2}\right), 29.0\left(\mathrm{CH}_{2}\right)$, $26.6\left(\mathrm{CH}_{2}\right), 26.2\left(\mathrm{CH}_{2}\right), 26.1\left(\mathrm{CH}_{2}\right)$. IR (solid, cm $\left.{ }^{-1}\right)$ : $3433(\mathrm{O}-\mathrm{H}), 3031(\mathrm{C}-\mathrm{H}), 2952(\mathrm{C}-\mathrm{H})$, 2928 (C-H), 2916 (C-H), 2893 (C-H), 2872 (C-H), 2849 (C-H), 1493, 1442, 1400, 1352, 1323, 1284, 1271, 1263, 1210, 1200, 1178, 1128, 1086, 1071, 1029, 1021, 1007. HRMS (EI): Calcd. for $\mathrm{C}_{13} \mathrm{H}_{18} \mathrm{O} m / z=190.1353$, found $m / z=190.1354[\mathrm{M}]^{+}$. Melting Point: $72{ }^{\circ} \mathrm{C} .[\alpha]_{\mathbf{D}}{ }^{20}:+37.8(\mathrm{c}=1.0$, $\left.\mathrm{CHCl}_{3}\right) . \mathbf{R}_{\mathbf{f}}($ EtOAc $:$ hexane $=1: 19)=0.21$. HPLC: Chiralpak ID-3 (hexane $: 2-\mathrm{PrOH}=95: 5$, flow rate $1.0 \mathrm{~mL} / \mathrm{min}, \lambda=210 \mathrm{~nm}$ ), retention times $t_{\mathrm{R}}($ minor $)=4.3 \mathrm{~min}, t_{\mathrm{R}}$ (major) $=5.5 \mathrm{~min}$. Analytical data are in agreement with literature data. ${ }^{\mathrm{S}, 5}$

Figure S77: ${ }^{1} \mathrm{H}$ NMR spectrum of enantioenriched $(R)-\mathbf{1 1 h}\left(300 \mathrm{MHz}, \mathrm{CDCl}_{3}\right)$.

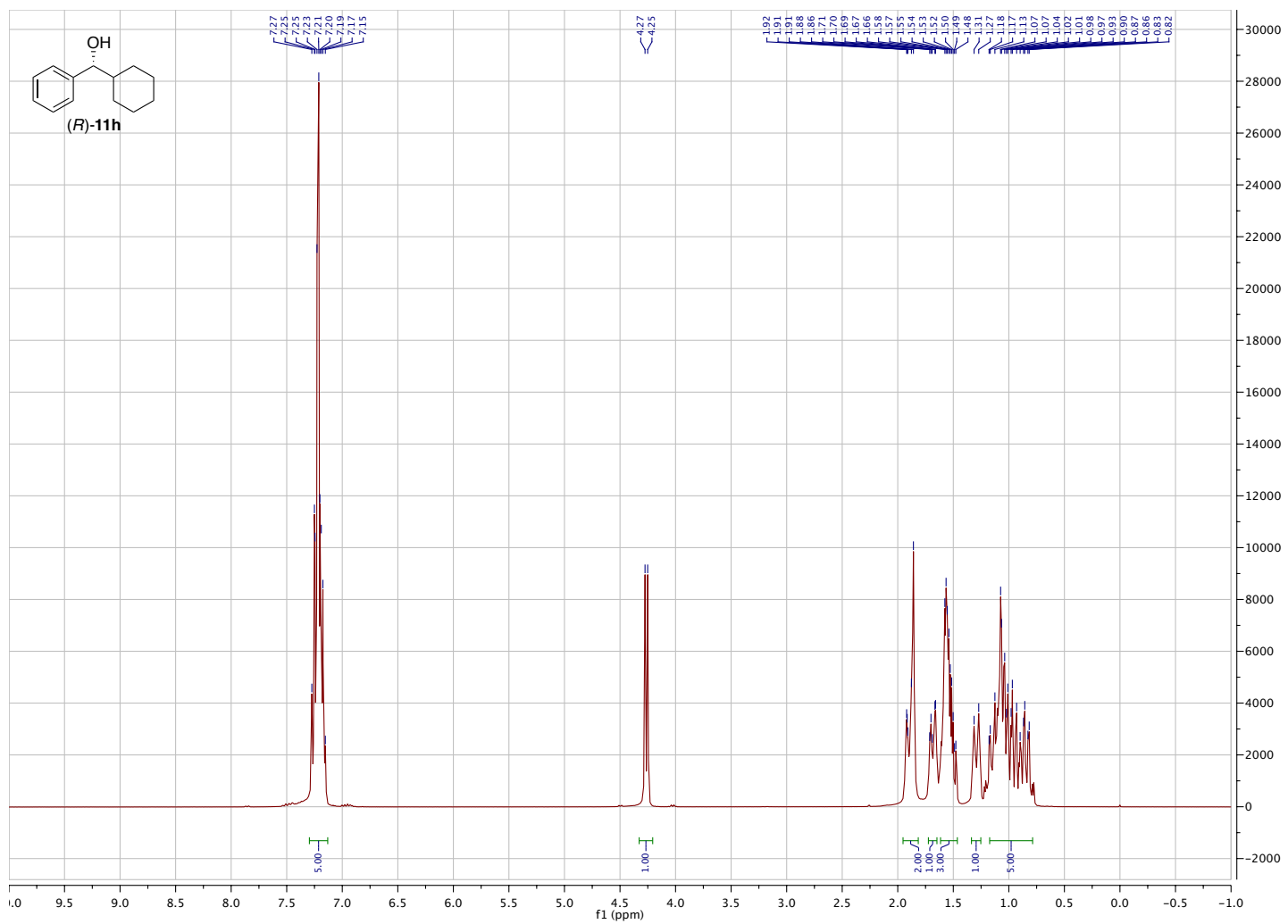


Figure S78: ${ }^{13} \mathrm{C}\left\{{ }^{1} \mathrm{H}\right\}$ NMR spectrum of enantioenriched $(R)-\mathbf{1 1 h}\left(75 \mathrm{MHz}, \mathrm{CDCl}_{3}\right)$.

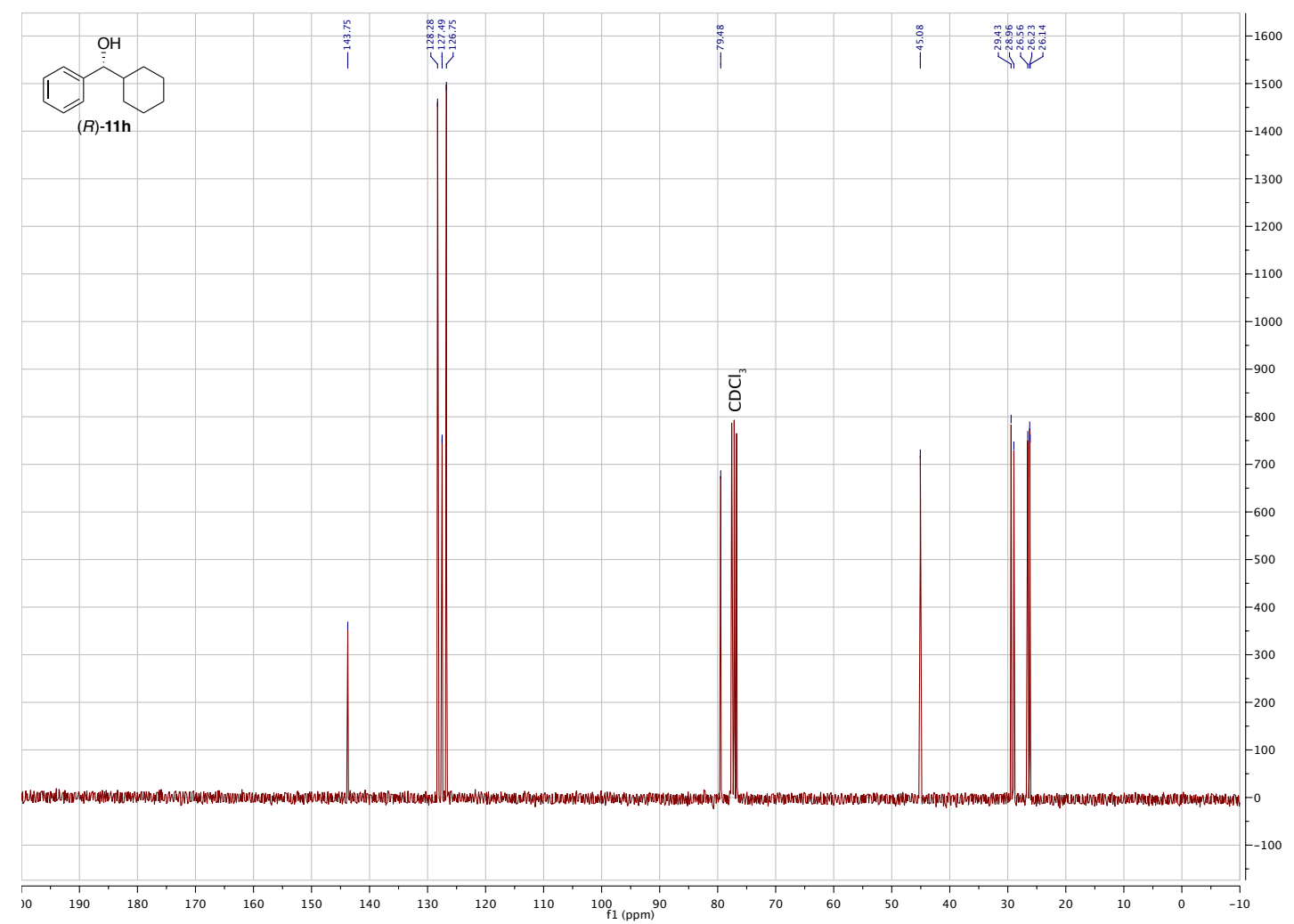

Figure S79: HPLC trace of enantioenriched $(R)-\mathbf{1 1 h}$ (obtained with catalyst 9b).

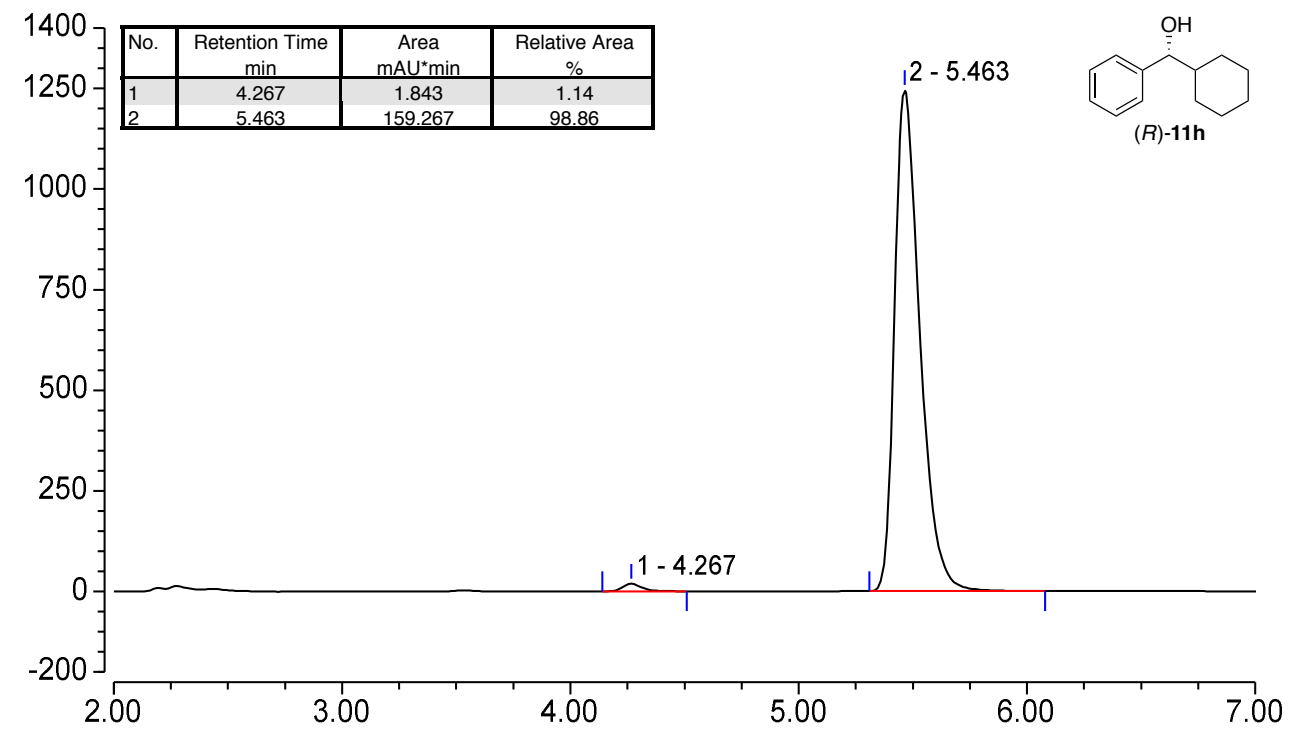


Figure S80: HPLC trace of racemic 11h.
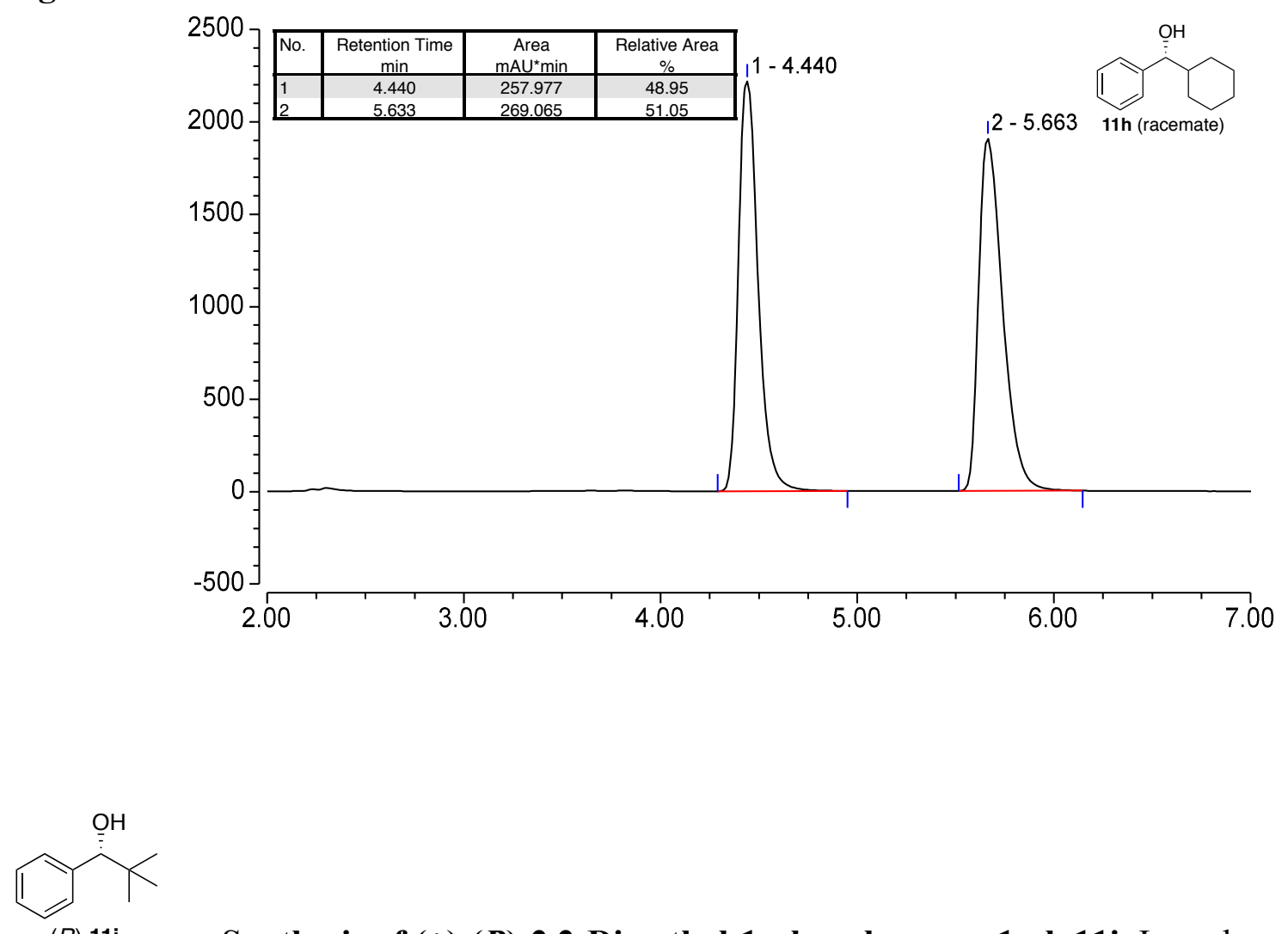

Synthesis of $(+)-(R)-2,2-D i m e t h y l-1-p h e n y l p r o p a n-1-o l, 11 i$. In a glove box, a vial was charged with complex 9b (204 mg, $200 \mu \mathrm{mol}, 0.2 \mathrm{~mol} \%)$ and sodium tert-butoxide (192 mg, $2.00 \mathrm{mmol}, 2.0 \mathrm{~mol} \%$ ). The vial was closed with a plastic cap and removed from the glove box. The solids were added to a flame-dried $1000 \mathrm{~mL}$ two-neck round-bottom flask with reflux condenser using a paper funnel and dried for 5 min under high vacuum. 2-Propanol (250 mL) was added using a Teflon cannula, and the flask was immersed in a preheated oil bath at $75{ }^{\circ} \mathrm{C}$. After 5 min, 2-propanol (250 mL) was added using a Teflon cannula. After additional $5 \mathrm{~min}$, tert-butyl(phenyl)methanone $(\mathbf{1 0 i})^{\mathrm{S} 6}(16.7 \mathrm{~mL}, 100 \mathrm{mmol})$ was added, and the solution was stirred at $75^{\circ} \mathrm{C}$ for $5.0 \mathrm{~h}$. The solvent was removed at the rotary evaporator $\left(40{ }^{\circ} \mathrm{C}, 90 \mathrm{mbar}\right)$, and the crude product was purified by flash column chromatography on silica gel $\left(\mathrm{Et}_{2} \mathrm{O}:\right.$ pentane $=0: 1$ to $\left.1: 1\right)$ to afford the product as an off-white solid. Yield: $16.03 \mathrm{~g}(97.6 \%, 95.8 \%$ ee $) .{ }^{1} \mathbf{H} \mathbf{N M R}\left(300 \mathrm{MHz}, \mathrm{CDCl}_{3}\right)$ : $\delta 7.36-7.23(m, 5 \mathrm{H}, \mathrm{Ar}-H), 4.39(s, 1 \mathrm{H}, \mathrm{CHOH}), 1.93($ br $s, 1 \mathrm{H}, \mathrm{OH}), 0.94\left(s, 9 \mathrm{H}, \mathrm{C}\left(\mathrm{CH}_{3}\right)_{3}\right)$. 
${ }^{13} \mathbf{C}\left\{{ }^{1} \mathbf{H}\right\}$ NMR (75 MHz, $\mathrm{CDCl}_{3}$ ): $\delta 142.3$ (arom.), 127.73 (arom.), 127.67 (arom.), 127.4 (arom.),

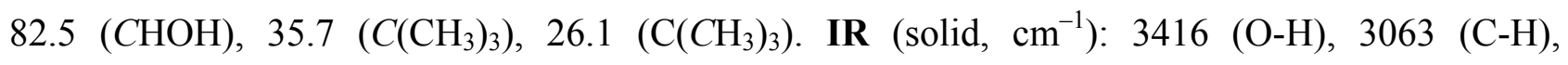
3035 (C-H), 2950 (C-H), 2929 (C-H), 2901 (C-H), 2866 (C-H), 1603, 1491, 1479, 1451, 1402, $1385,1364,1352,1304,1285,1236,1220,1201,1178,1082,1043,1027,1001$. HRMS (EI): Calcd. for $\mathrm{C}_{11} \mathrm{H}_{16} \mathrm{O} m / z=164.1196$, found $m / z=164.1198[\mathrm{M}]^{+}$. Melting Point: $56{ }^{\circ} \mathrm{C}$. $[\boldsymbol{\alpha}]_{\mathbf{D}}{ }^{20}:+36.1\left(\mathrm{c}=1.0, \mathrm{CHCl}_{3}\right) . \mathbf{R}_{\mathbf{f}}\left(\mathrm{Et}_{2} \mathrm{O}:\right.$ pentane $\left.=1: 9\right)=0.34$. HPLC: Chiralpak IC-3 (hexane : $2-\mathrm{PrOH}=95: 5$, flow rate $1.0 \mathrm{~mL} / \mathrm{min}, \lambda=210 \mathrm{~nm}$ ), retention times $t_{\mathrm{R}}$ (major) $=3.8 \mathrm{~min}$, $t_{\mathrm{R}}($ minor $)=4.3 \mathrm{~min}$. Analytical data are in agreement with literature data. ${ }^{\mathrm{S}, 5}$

Figure S81: ${ }^{1} \mathrm{H}$ NMR spectrum of enantioenriched $(R)-11 i\left(300 \mathrm{MHz}, \mathrm{CDCl}_{3}\right)$.

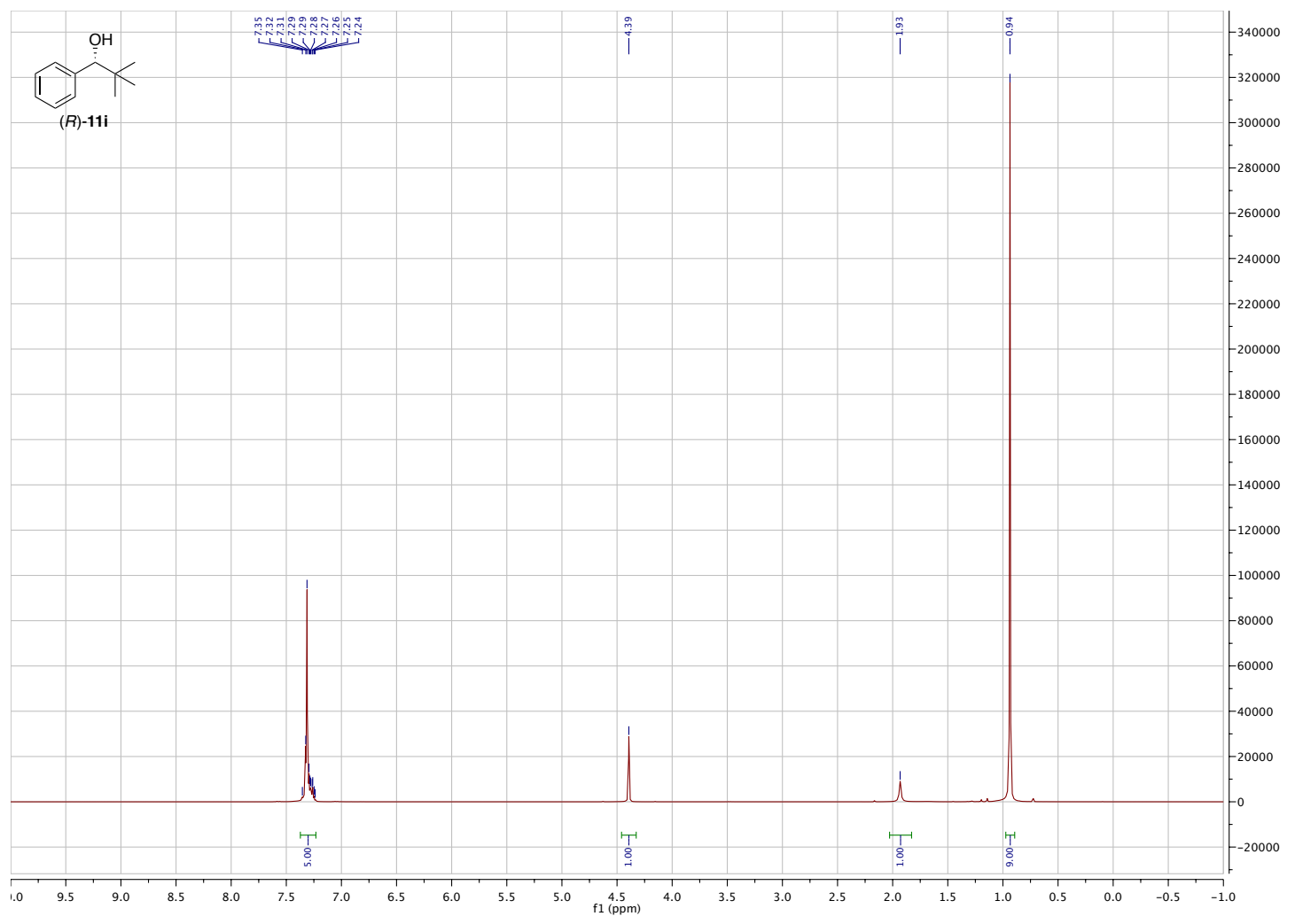


Figure S82: ${ }^{13} \mathrm{C}\left\{{ }^{1} \mathrm{H}\right\}$ NMR spectrum of enantioenriched $(R)-11 \mathbf{i}\left(75 \mathrm{MHz}, \mathrm{CDCl}_{3}\right)$.

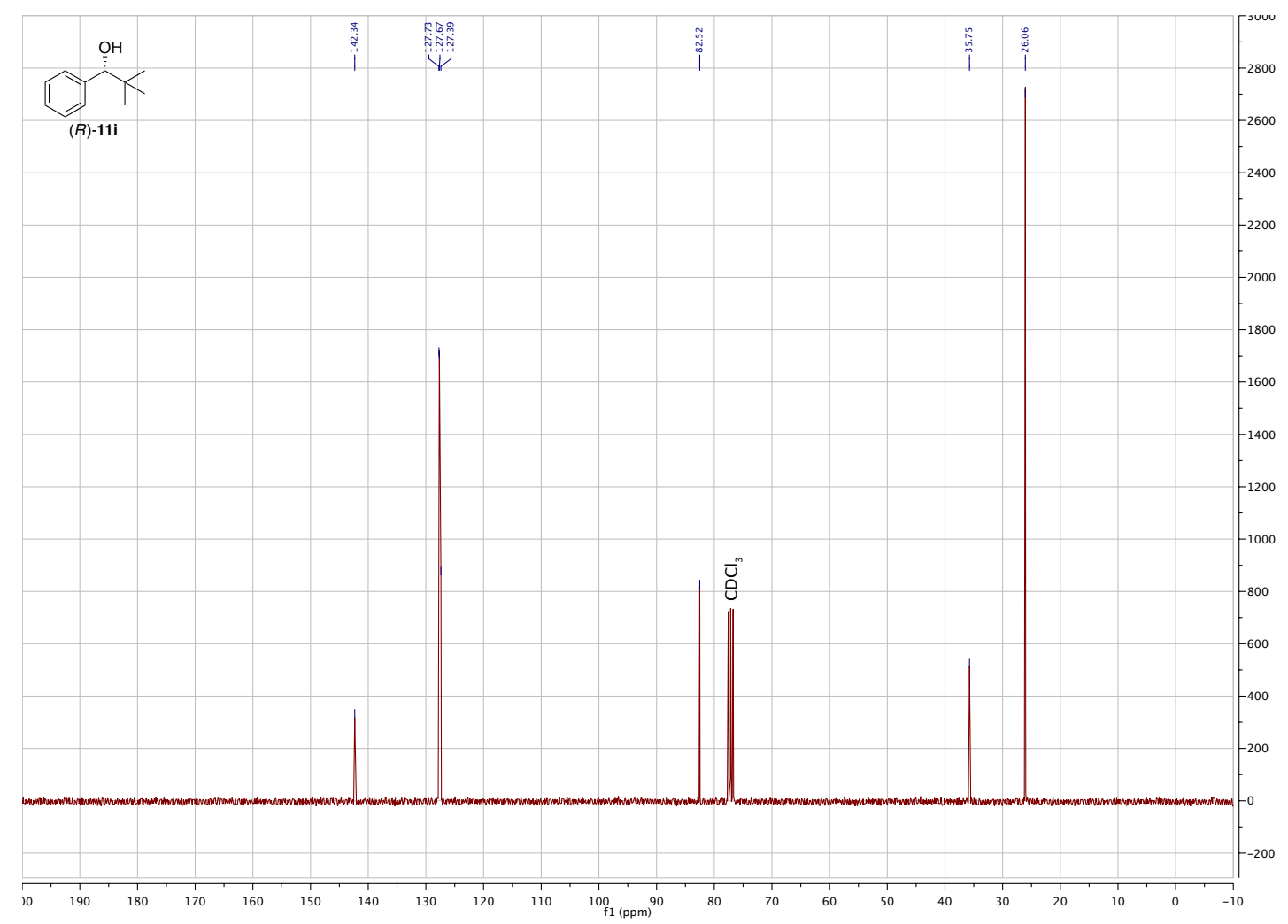

Figure S83: HPLC trace of enantioenriched $(R)-11 \mathbf{i}$ (obtained with catalyst $9 \mathbf{b}$ ).

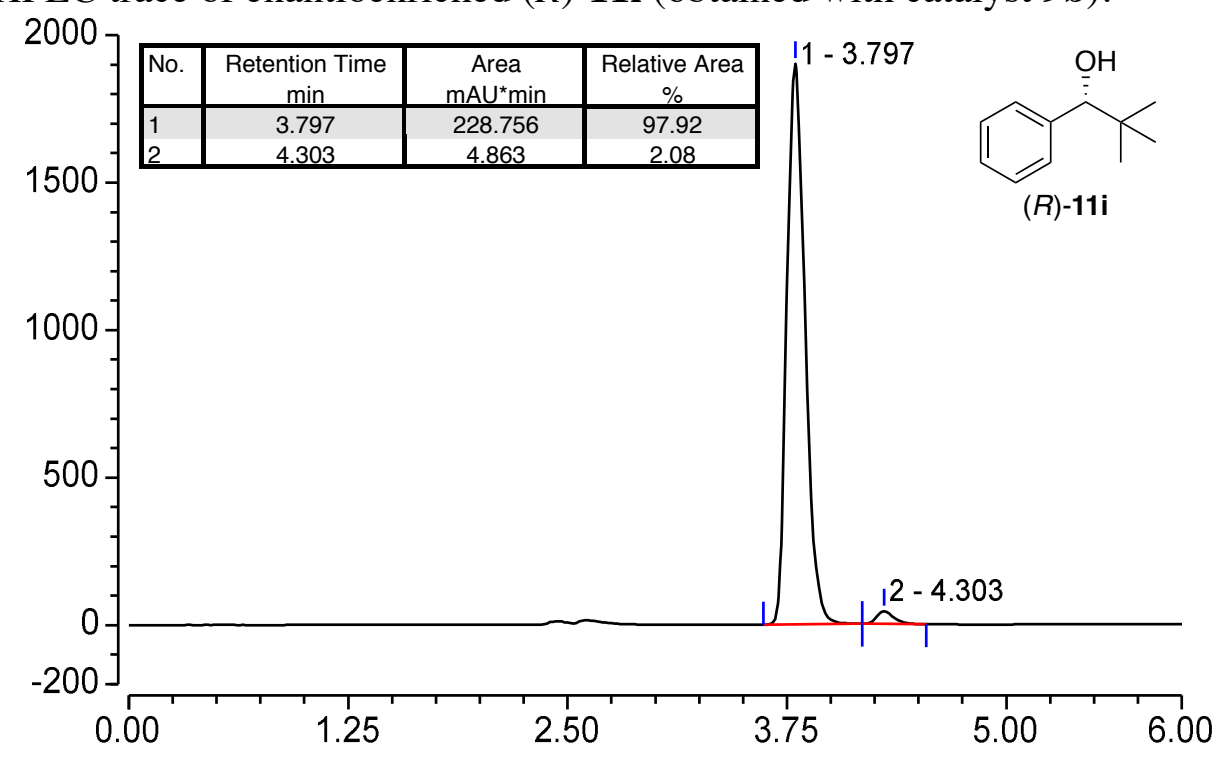


Figure S84: HPLC trace of racemic 11i.
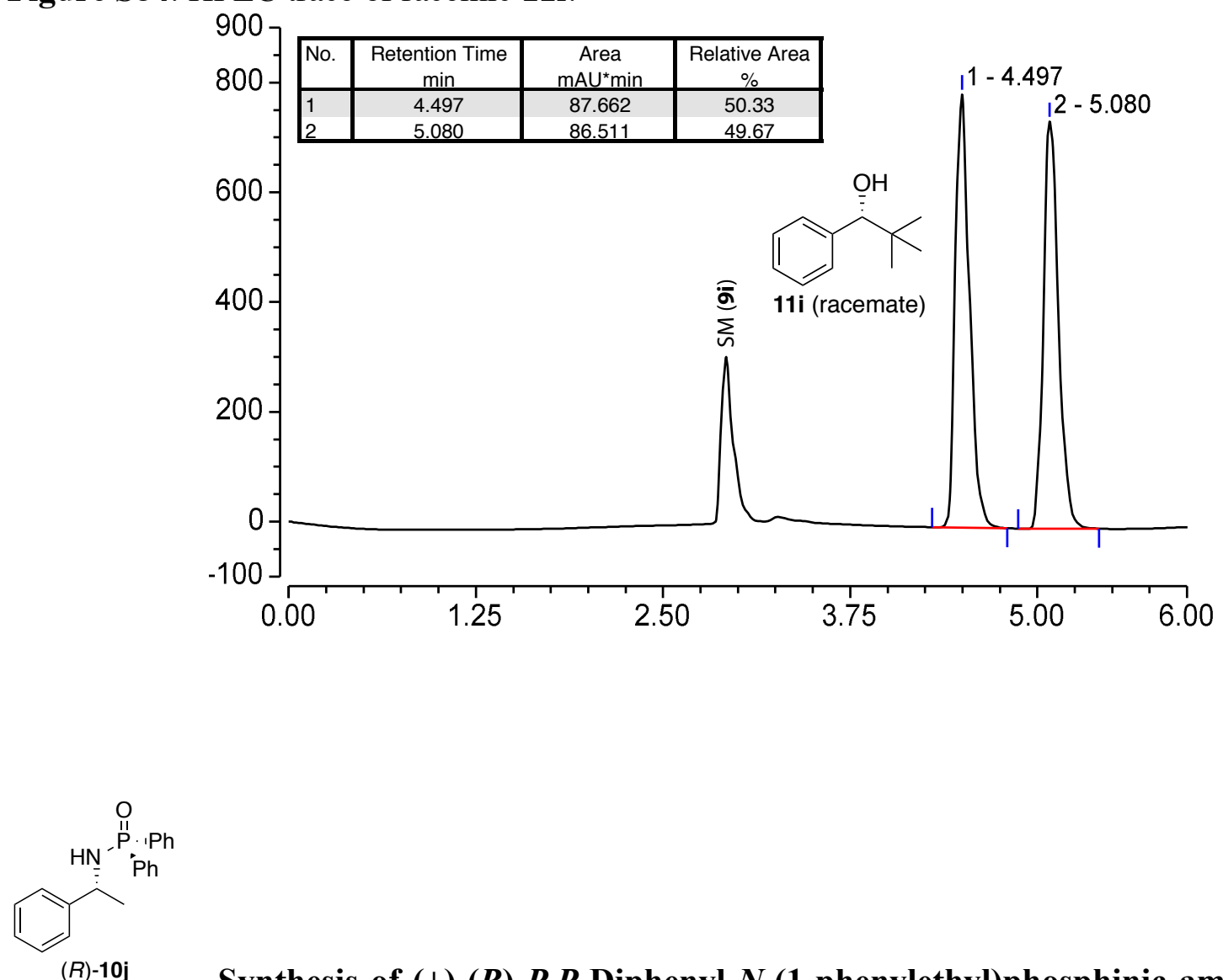

Synthesis of (+)-(R)-P,P-Diphenyl- $N$-(1-phenylethyl)phosphinic amide, $11 \mathrm{j}$. In a glove box, a vial was charged with complex 9a (203 mg, $200 \mu \mathrm{mol}, 0.2 \mathrm{~mol} \%)$ and sodium tertbutoxide (192 mg, $2.00 \mathrm{mmol}, 2.0 \mathrm{~mol} \%$ ). The vial was closed with a plastic cap and removed from the glove box. The solids were added to a flame-dried $1000 \mathrm{~mL}$ two-neck round-bottom flask with reflux condenser using a paper funnel and dried for 5 min under high vacuum. 2-Propanol (250 mL) was added using a Teflon cannula, and the flask was immersed in a preheated oil bath at $75{ }^{\circ} \mathrm{C}$. After $5 \mathrm{~min}$, 2-propanol $(250 \mathrm{~mL})$ was added using a Teflon cannula. After additional $5 \mathrm{~min}$, (E)-P,P-diphenyl- $N$-(1-phenylethylidene)phosphinic amide $(\mathbf{1 0 j})^{\mathrm{S} 7}(31.93 \mathrm{~g}, 100 \mathrm{mmol})$ was added, and the solution was stirred at $75^{\circ} \mathrm{C}$ for $5.0 \mathrm{~h}$ (Note: The reaction quickly turns dark green). The solvent was removed at the rotary evaporator $\left(40{ }^{\circ} \mathrm{C}, 90 \mathrm{mbar}\right)$. The crude product was suspended in 2-propanol $(200 \mathrm{~mL})$, and the solvent was removed at the rotary evaporator $\left(40{ }^{\circ} \mathrm{C}, 90 \mathrm{mbar}\right)$. 
This process was repeated once to assure complete removal of acetone. The crude product was dissolved in hot 2-propanol $(200 \mathrm{~mL})$ using a heat gun and filtered whilst hot. The filter was washed once with hot 2-propanol $(50 \mathrm{~mL})$. The solution was cooled to room temperature and placed in a freezer overnight. The precipitated solid was filtered off and washed with ice-cold 2-propanol $(3 \times 30 \mathrm{~mL})$ to afford the title product as beige crystals. Yield: $28.26 \mathrm{~g}(87.9 \%,>99.9 \% e e)$. The mother liquor was concentrated to ca. $30 \mathrm{~mL}$ and crystallized as described above to afford a second crop of crystals. Yield: $1.95 \mathrm{~g}(6.1 \%, 99.8 \% e e) .{ }^{1} \mathbf{H}$ NMR $\left(300 \mathrm{MHz}, \mathrm{CDCl}_{3}\right): \delta 8.00-7.78(m$, $4 \mathrm{H}, \mathrm{Ar}-H), 7.52-7.21(m, 11 \mathrm{H}, \mathrm{Ar}-H), 4.41\left(d d q,{ }^{3} J_{\mathrm{P}, \mathrm{H}}=9.8 \mathrm{~Hz},{ }^{3} J_{\mathrm{H}, \mathrm{H}^{\prime}}=9.8,6.8 \mathrm{~Hz}, 1 \mathrm{H}, \mathrm{C} H \mathrm{NH}\right)$, $3.50\left(d d,{ }^{3} J_{\mathrm{H}, \mathrm{H}^{\prime}}=9.8 \mathrm{~Hz},{ }^{2} J_{\mathrm{P}, \mathrm{H}}=6.2 \mathrm{~Hz}, 1 \mathrm{H}, \mathrm{N} H\right), 1.59\left(d,{ }^{3} J_{\mathrm{H}, \mathrm{H}^{\prime}}=6.8 \mathrm{~Hz}, 1 \mathrm{H}, \mathrm{CH}_{3}\right) .{ }^{31} \mathbf{P}\left\{{ }^{1} \mathbf{H}\right\} \mathbf{N M R}$ (122 MHz, $\left.\mathrm{CDCl}_{3}\right): \delta 22.4(s) .{ }^{13} \mathbf{C}\left\{{ }^{1} \mathbf{H}\right\}$ NMR $\left(75 \mathrm{MHz}, \mathrm{CDCl}_{3}\right): \delta 145.1\left(d,{ }^{3} J_{\mathrm{P}, \mathrm{C}}=6.6 \mathrm{~Hz}\right.$, arom.), $133.6\left(d,{ }^{1} J_{\mathrm{P}, \mathrm{C}}=74.3 \mathrm{~Hz}\right.$, arom. $), 132.4\left(d,{ }^{2} J_{\mathrm{P}, \mathrm{C}}=9.5 \mathrm{~Hz}\right.$, arom. $), 131.93\left(d,{ }^{1} J_{\mathrm{P}, \mathrm{C}}=77.0 \mathrm{~Hz}\right.$, arom.), $131.89\left(d,{ }^{2} J_{\mathrm{P}, \mathrm{C}}=9.4 \mathrm{~Hz}\right.$, arom. $), 131.7\left(d,{ }^{4} J_{\mathrm{P}, \mathrm{C}}=2.8 \mathrm{~Hz}\right.$, arom. $), 131.6\left(d,{ }^{4} J_{\mathrm{P}, \mathrm{C}}=2.8 \mathrm{~Hz}\right.$, arom.), 128.5 (arom.), 128.4 (d, ${ }^{3} J_{\mathrm{P}, \mathrm{C}}=7.0 \mathrm{~Hz}$, arom.), 128.3 ( $d,{ }^{3} J_{\mathrm{P}, \mathrm{C}}=6.5 \mathrm{~Hz}$, arom.), 127.0 (arom.), 126.0 (arom.), $51.0(\mathrm{CHNH}), 25.9\left(\mathrm{CH}_{3}\right)$. IR (solid, $\left.\mathrm{cm}^{-1}\right)$ : $3157(\mathrm{~N}-\mathrm{H}), 3054(\mathrm{C}-\mathrm{H}), 3029$ (C-H), 2973 (C-H), 2925 (C-H), 2868 (C-H), 1591, 1496, 1483, 1462, 1450, 1435, 1367, 1343, 1331, 1309, 1297, 1205, 1187, 1176, 1125, 1108, 1083, 1069, 1036, 1025. HRMS (ESI): Calcd. for $\mathrm{C}_{20} \mathrm{H}_{21} \mathrm{NOP} m / z$ 322.1355, found $m / z 322.1358[\mathrm{M}+\mathrm{H}]^{+}$. Melting Point: $195{ }^{\circ} \mathrm{C} .[\boldsymbol{\alpha}]_{\mathbf{D}}{ }^{20}:+33.8$ $\left(\mathrm{c}=1.0, \mathrm{CHCl}_{3}\right)$. HPLC: Chiralpak OD-H (hexane : 2-propanol, $90: 10$, flow rate $=1.0 \mathrm{~mL} / \mathrm{min}$, $\lambda=230 \mathrm{~nm})$, retention times $t_{\mathrm{R}}($ major $)=10.1 \mathrm{~min}, t_{\mathrm{R}}($ minor $)=13.1 \mathrm{~min}$. Analytical data are in agreement with literature data. ${ }^{\mathrm{S}}$ 
Figure S85: HPLC trace of enantioenriched $(R)-\mathbf{1 1} \mathbf{j}$ (first fraction, obtained with catalyst 9a).

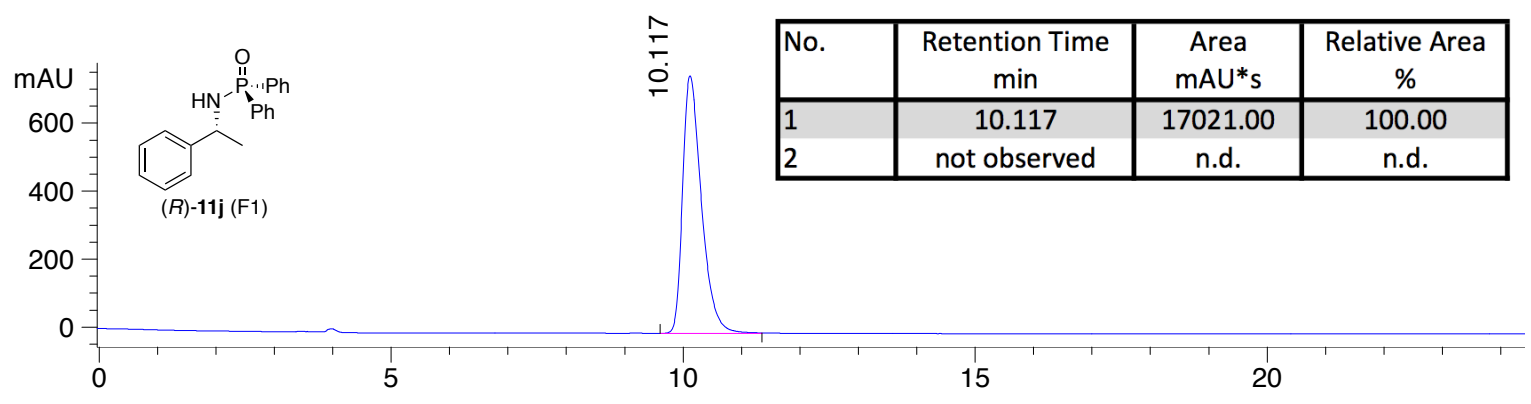

Figure S86: HPLC trace of enantioenriched $(R)-\mathbf{1 1} \mathbf{j}$ (second fraction, obtained with catalyst $\mathbf{9 a}$ ).

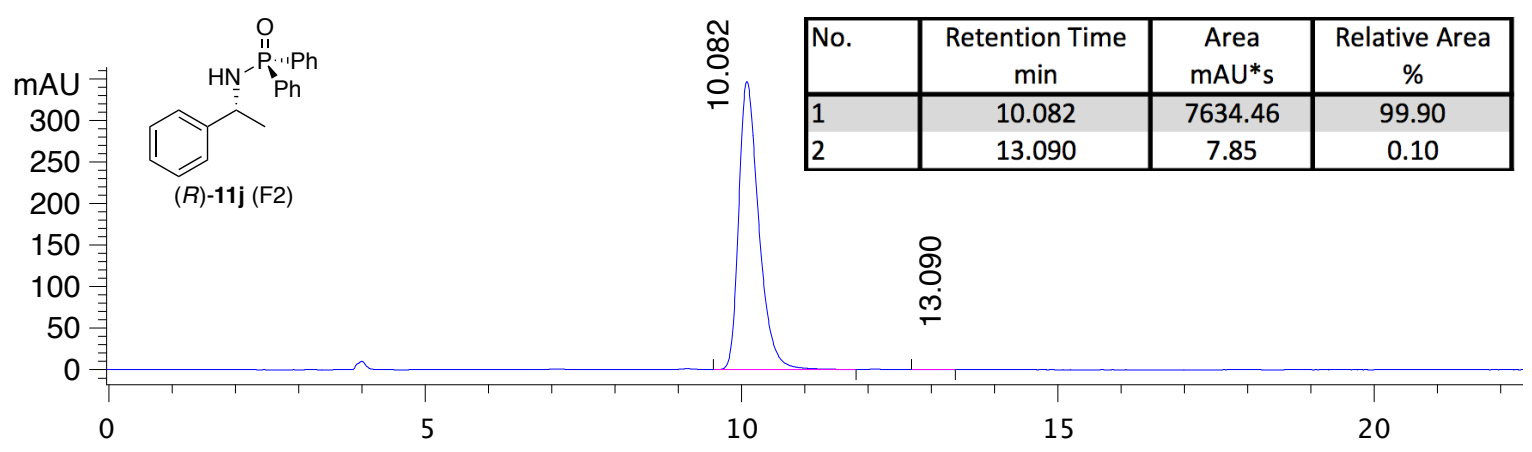

Figure S87: HPLC trace of racemic 11j.

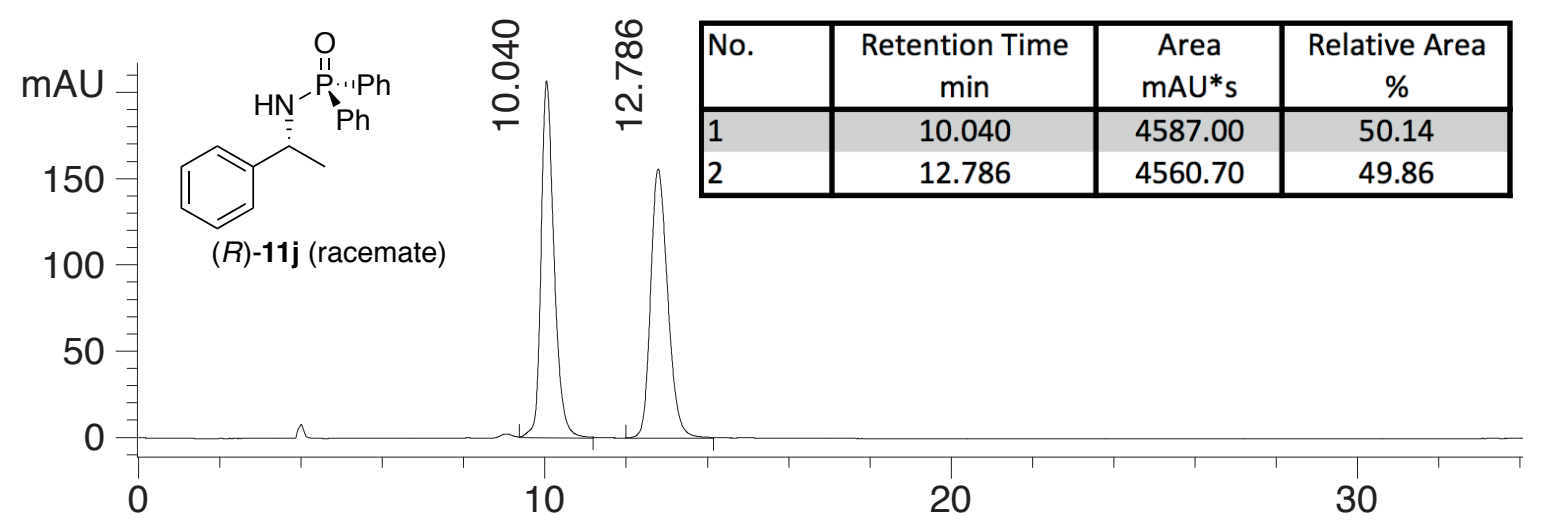


Figure S88: ${ }^{1} \mathrm{H}$ NMR spectrum of enantioenriched $(R)-\mathbf{1 1} \mathbf{j}\left(300 \mathrm{MHz}, \mathrm{CDCl}_{3}\right)$.

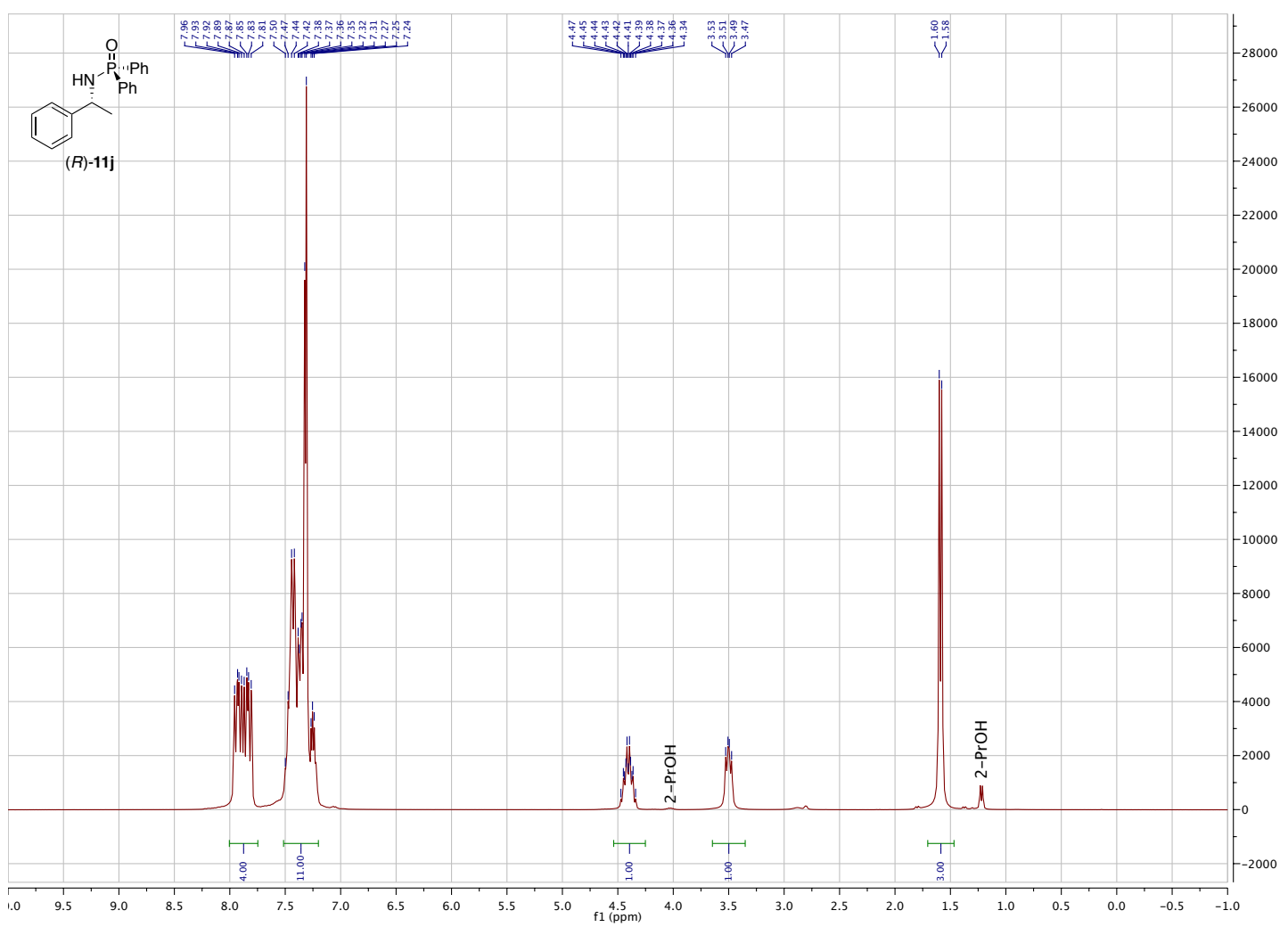

Figure S89: ${ }^{31} \mathrm{P}\left\{{ }^{1} \mathrm{H}\right\} \mathrm{NMR}$ spectrum of enantioenriched $(R)-\mathbf{1 1} \mathbf{j}\left(122 \mathrm{MHz}, \mathrm{CDCl}_{3}\right)$.

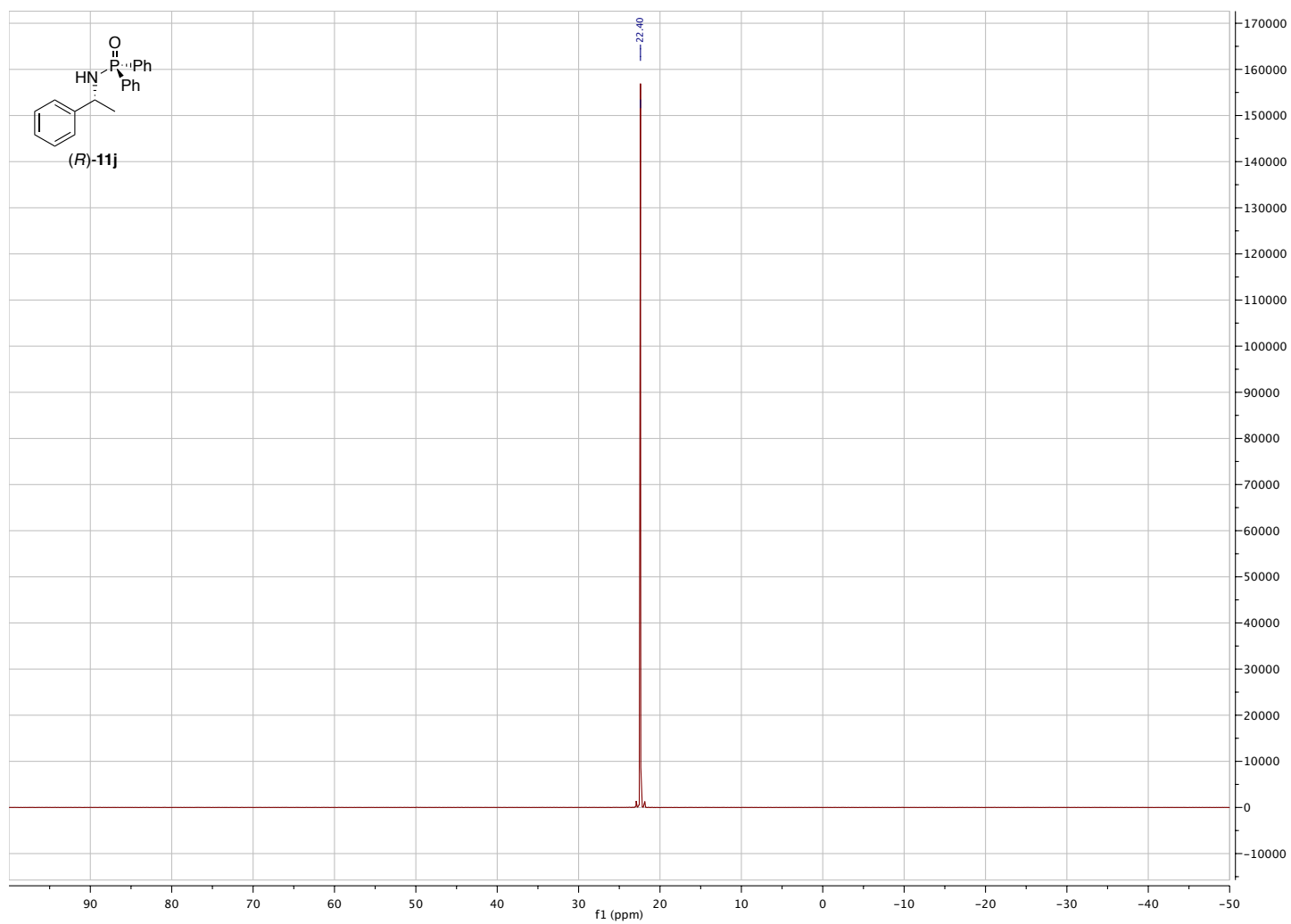


Figure S90: ${ }^{13} \mathrm{C}\left\{{ }^{1} \mathrm{H}\right\}$ NMR spectrum of enantioenriched $(R)-\mathbf{1 1} \mathbf{j}\left(75 \mathrm{MHz}, \mathrm{CDCl}_{3}\right)$.

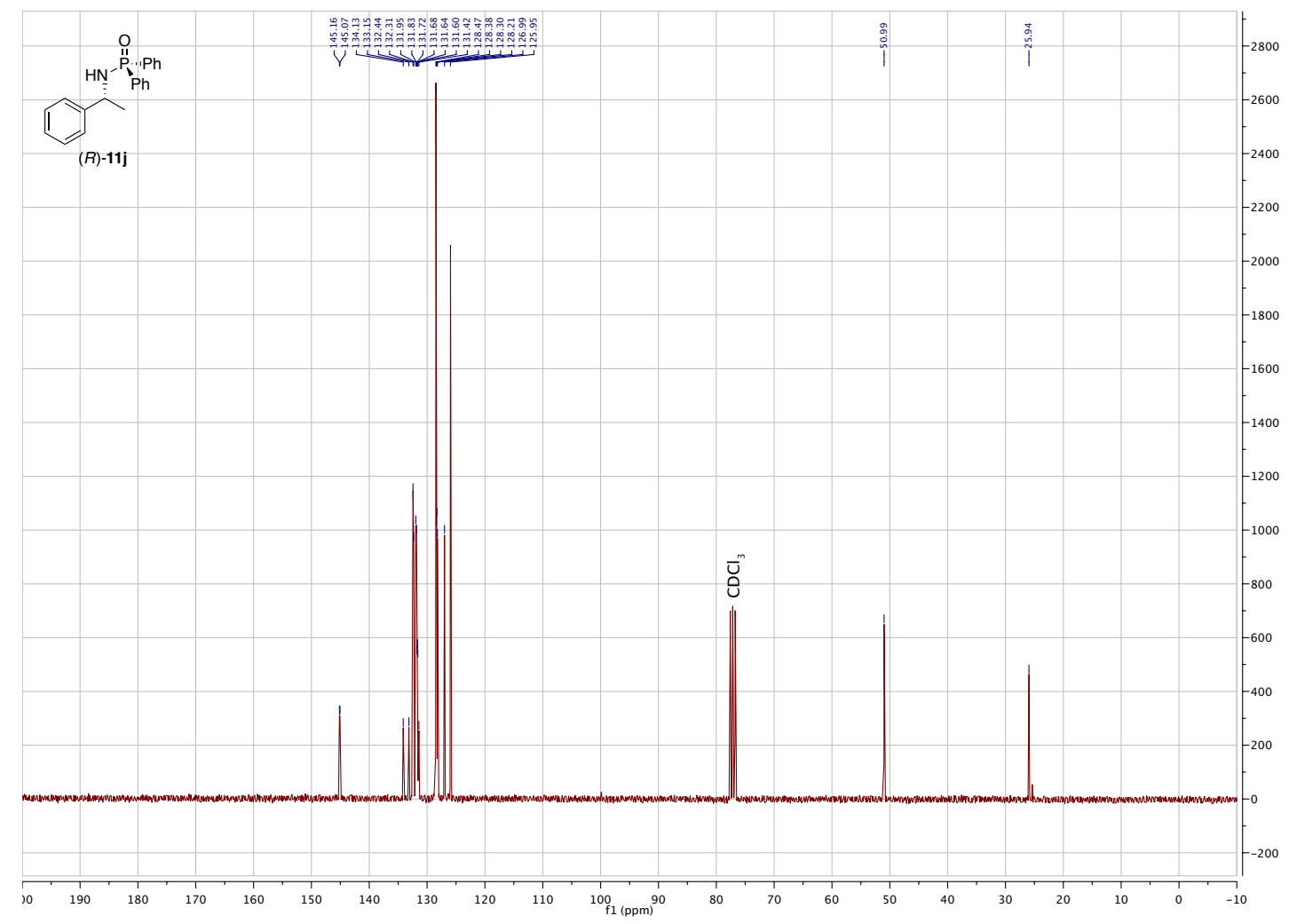

\section{References}

(S1) Li, Z.; Kumar, A.; Vachhani, D. D.; Sharma, S. K.; Parmar, V. S.; Van der Eycken, E. V. Eur. J. Org. Chem. 2014, 2084.

(S2) Wang, W.-M.; Liu, L.-J.; Zhao, C.-Q.; Han, L.-B. Eur. J. Org. Chem. 2015, 2342.

(S3) Bigler, R.; Otth, E.; Mezzetti, A. Organometallics 2014, 33, 4086.

(S4) Bigler, R.; Huber, R.; Mezzetti, A. Angew. Chem. Int. Ed. 2015, 54, 5171.

(S5) Bigler, R.; Mezzetti, A. Org. Lett. 2014, 16, 6460.

(S6) Pearson, D. E. J. Am. Chem. Soc. 1950, 72, 4169.

(S7) Vyas, D. J.; Fröhlich, R.; Oestreich, M. Org. Lett. 2011, 13, 2094. 